LBL 32400

\title{
Nucleation and Growth in Electrodeposition of Thin Copper Films on Pyrolytic Graphite
}

\author{
Filiz S. Kinaci \\ (Master's Thesis) \\ with Rolf $H$, Muller \\ May 1992 \\ Material Sciences Division \\ Lawrence Berkeley Laboratory \\ and \\ Department of Chemical Engineering \\ University of California \\ Berkeley, California
}

This work. was supported by the Director, Office of Energy Research, Office of Basic Energy Sciences, Materials Sciences Division of the U.S. Department of Energy under contract No. DE-AC03-76SF00098

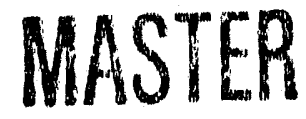


In memory of my grandparents,

Bahri and Nilüfer Kunack 


\section{CONTENTS}

List of Figures: ........................................................................... vi

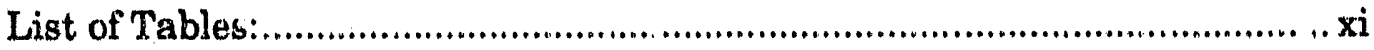

List of Symbols: .............................................................................. xii

Ácknowledgements: ...................................................................... xvi

\section{Chapter 1: Introduction and Literature Review}

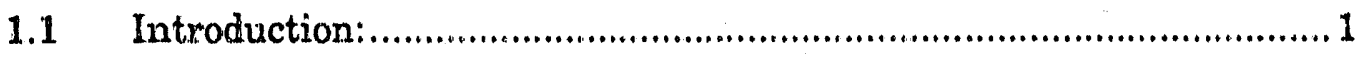

Applications: ................................................................ 6

1.2 Nucleation and Growth Theories: ............................................... 7

Fleischmann and Thir'sk: ......................................................7

Kaishev and Mutaftshiev: .....................................................8

Markov and Stoycheva:....................................................... 9

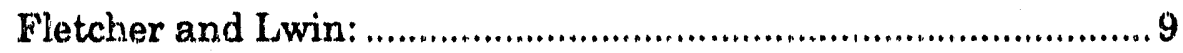

Growth Mechanisms: ........................................................ 10

1.3 Techniques for Analysis of Nucleation: ......................................... 11

1.4 Properties of Highly Oriented Pyrolytic Graphite: .......................... 16

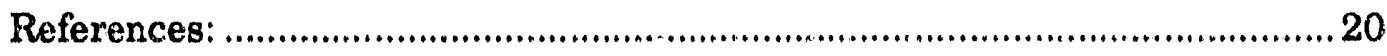

\section{Chapter 2: Determination of Nucleation Density}

2.1 General Experimental Setup:..................................................24

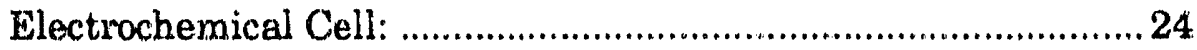

Data Collection Equipment: ................................................25

Surface Analysis Techniques: ................................................27

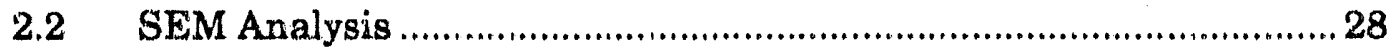

Experimental Reproducibility: .............................................. 30

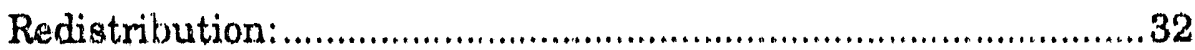


2.3 Scharifker and Mostany's Model: ...................................................35

Description of Model: ..................................................................35

Comparison with Experimental Measurement: ........................ 38

2.4 Scanning Tunneling Microscopy: ............................................42 42

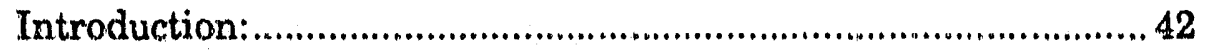

Piezoelectric Scanning Mechanism: .........................................43

Data Collection and Presentation: ..............................................44

Experimental Techniques: ..................................................45

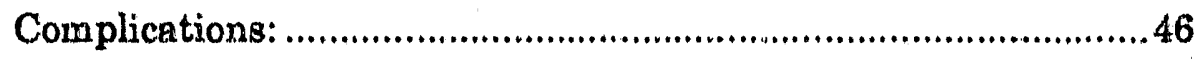

Image interpretation: .......................................................47

Experimental Results: Bare HOPG:.....................................50

HOPG with copper deposit: ..................53

2.5 Ramax Spectroscopy: ...............................................................55

Introduction to Raman Spectroncopy: ....................................55

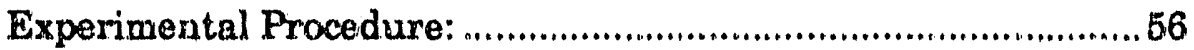

Raman Models: ....................................................................66

Simple Geometric Model: ............................................69

Effective Film Models:................................................ 73

Effective Medium Approximation Model: ................... 77

Island Model: .......................................................... 78

Limitations of Raman as a Predictive Tool:............................... 79

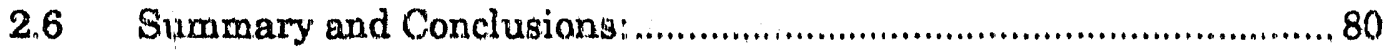

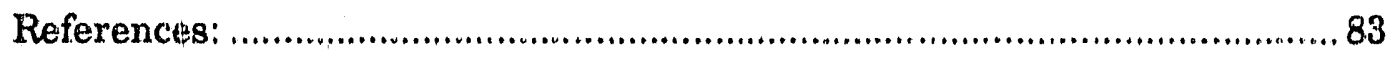

\section{Chapter 3: Electrochemical Analysis}

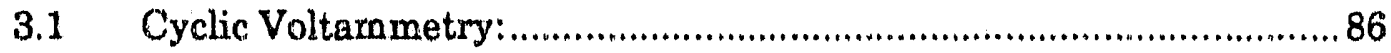

Experimental Procedure: .................................................86

Comparison of Results: ............................................... 86 
3.2. Controlling and Increasing the Nucleation Density; ........................90

Effect of Current Density: ........................................................99

Effect of Potential: ...............................................................93

Effect of Duration: ...................................................................97

Effect of Concentration: ......................................................... 100

Effect of Repeated Pulse Experiments: ................................... 102

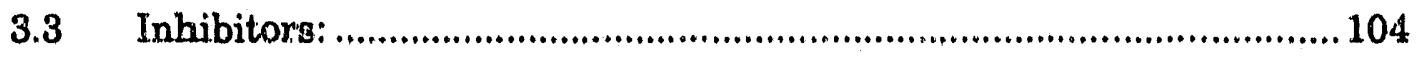

Introduction to inhibitors:..................................................... 104

Conclusions from Armstrong's work: ...................................... 106

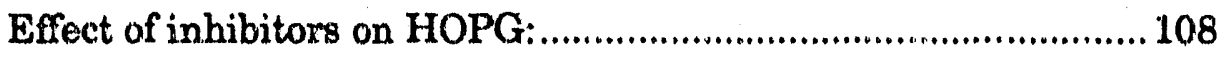

Potential step experiments: ................................................... 114

3.4 Summary and Conclusions: ..................................................... 116

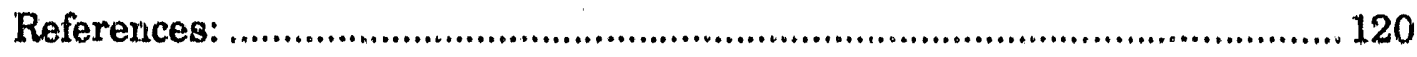

\section{Chapter 4: Conducting Polymer Electrodes}

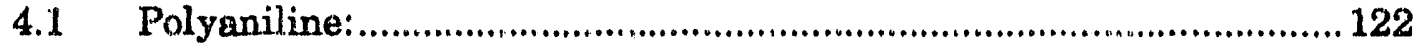

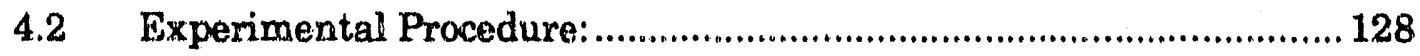

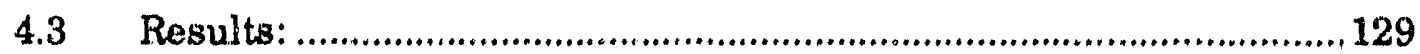

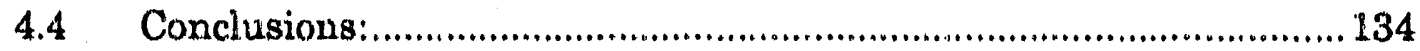

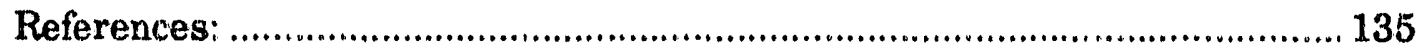

\section{Chapter 5: Summary and Conclusions}

5.1 Determination of Nucleation Density: .......................................... 138

$5.2 \quad$ Experimental Results: ............................................................ 140

5.3 Possible Extensions of Present Study:........................................... 141

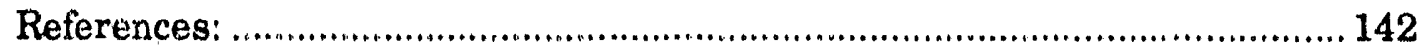

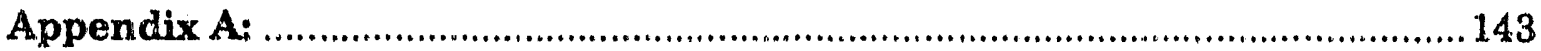

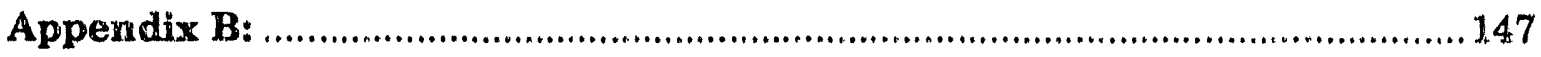




\section{List of Figures:}

\section{Chapter One:}

1.1 SEM of typical copper depasit on HOPG XBB 925-3204

1.2 Comparison of high and low nucleation XBl, 925-1100

1-3 Comparison of instantaneous and progressive nucleation XBL 925-1101

1.4 Possible mechunisms for electrochemical growth XBL 925-1.102

1.5 Crystalline structure of graphite XBL 925.1103

\section{Chapter Two:}

2-1 Schematic of three-electrode electrochemical cell XBL 925-1104

2-2 Equipment set-up with 173 potentiostat XBL 925-1105

2-3 Equipment set-up with 273 potentiostat XBL $925-1106$

2-4 SEM of typical copper deposit on HOPG XBB 925-3204

2-5 SEM of defect decoration

XBB 916-4661

2-6 Open circuit potential following copper deposition on graphite XBL, 925-1107

2.7 Open circuit potential following copper deposition on copper XOBL 925-1108 
2-8 Experimentally measured current transient and Scharifker model fit XBL $925 \cdot 1109$

2-9 Instantaneous and progressive nucleation current transients XBL $925-1110$

2-10 Piezo drive for the scanning tunneling microscope XBL 925-1111

2-11 Drawing of multiple tunneling on an STM tip XBL 917-1639

2-12 STM image of the surface imaging the tip XBB 917-6264A

2-13 Atomic resolution STM of HOPG atoms in air XBB 916.4666A

2-14 Atomic resolution STM of HOPG atoms under water XBB 925-3226

2-15 Drawing of the visible and invisible graphite atoms in STM XBI, 925-1112

2-16 STM and SEM of copper nodules on HOPG XBB 916-4663A XBB 916-4658A

2-17 Raman spectra of carbon allotropes XBL $925 \cdot 1113$

2-18 Raman experimental setup XBL 925-1114

2-19 Photograph of electrochemical cell XBB 925-3302

2-20 Experimental sequence for Raman experiments XBL 925-1115

2-21 Sequential Raman spectra from single experiment XIs, $925-1116$ 
2-22 Relative Raman versus charge data for four data sets XBL 925-1117

2-23 Schematic of Optical Models XBL 925- 1118

2-24 Linear dependence between nucleation densities determined by SEM and those predicted by the simple geometrical model XBL 925-1119

2.25 Definition of refractive index, transmission coefficient, reflection coefficient and optical model thickness XBL 925-1120

2-26 Island model fit to the sequential data presented in figure 2-21 XBL 925-1121

\section{Chapter Three:}

3-1 CV on platinum

XBL, 925-1122

3-2 CV on HOPG

XBL 925-1123

3-3 CV on HOPG without anodic sweep XBL 925-1124

3-4 Potential transient to a current step XBL 925-1125

3-5 SEM of damaged HOPG surface XBB 923-2082

3-6 Current transient to a potential step XBL 925-1126

3-7 Effect of potential on nucleation density XBL 925-1127

3-8 Effect of duration on nucleation density

XBL 925-1128 
3-9 SEIM results on the effect of duration $-700 \mathrm{mV}$ series XIBB 917-6265

3-10 Effect of cupric ion concentration on the nucleation density XBL, 925-11.29

3-11 Repeated pulse potential profile

XBL 925-1130

3-12 Comparison of SEM result

XBB 917-6263

3-13 Chemical formulae for BTA and PVA

XBL 925-1131

3-14 First and second sweeps of CV on HOPG

XBL $925-1132$

3-15 First and second sweeps of CV on HOPG in BTA containing electrolyte XBL $925 * 1133$

3-16 First and second sweeps of CV on HOPG in PVA containing electrolyte XBL 925.1134

3-17 SEM of HOPG surface after CV with PVA XBB 923-2201

3-18 SEM of renucleation in the presence of PVA XBB 923-2197

3-19 SEM of nuclei in the absence of PVA

XBB 925-3205

3-20 SEM (high magmification) of copper clusters XBB 923-2195

\section{Chapter Four:}

4-1 Oxidation states of polyaniline XBL 925-1135

4-2 Resonance stabilized radical cation precursor to polymerization reaction XBL 925-1136 
4-3 Possible polymerization mechanisms

XBL 925-1137

4-4 Cyclic voltammogram of aniline electropolymerization

XBL 925-1138

4-5 SEM of copper deposit on polyaniline

XBB 925-3206

4-6 Potential scale for standard reduction potentials of copper and rhodium with respect to PANI switching potential

XBL 925-1139 


\section{List of Tables:}

\section{Chapter Two:}

2-1 Results from sequential Raman spectra experiments

2-2 Simple geometric model results for a single set of experiments

2-3 Simple geometric model results compared with measured values

\section{Chapter Three:}

3-1 Results from galvanostatic step experiments

3.2 Results of CV experiments with inhibitors

3-3 Results from potential step experiments with inhibitors. 


\section{List of Symbols:}

A Nucleation rate per active site $\left(\mathrm{s}^{-1}\right)$

a $\mathrm{zFc}(\mathrm{D} / \pi)^{-1 / 2}$, substitution in the Scharifker and Mostany model

b

$N_{0} \pi k D$, substitution in the Scharifker and Mostany model

c

Bulk concentration $\left(\mathrm{mol} / \mathrm{cm}^{3}\right)$

D Diffusion coefficient $\left(\mathrm{cm}^{2} / \mathrm{s}\right)$

d Thickness of effective copper film

E

Amplitude of the electric field.

Einc

Amplitude of the incident electric field

$\mathbf{E}_{\text {inc,H }}$

Amplitude of incident electric field to HOPG

$\mathbf{E}_{\text {gcat,bare }}$

Amplitude of electric field of scattered light from a bare graphite surface

$\mathbf{E}_{\text {scat, }}$ An Amplitude of electric field of scattered light from a copper covered graphite surface

$\mathbf{E}_{\text {gcat,H }}$ Amplitude of the scattered electric field from HOPG

$\mathbf{f}_{1}, \mathbf{f}_{2} \quad$ Functions in equation $2-8$ and $2-9$

I

Current density $\left(\mathrm{A} / \mathrm{cm}^{2}\right)$

Ibaxe

Intensity of scattered light from bare graphite

ICu

Intensity of scattered light from copper covered graphite

Im Current density at maximum

M $_{\text {w }} \quad$ Molecular weight of deposit $(\mathrm{g} / \mathrm{mol})$

N Number density of nuclei (nuclei $/ \mathrm{cm}^{2}$ )

$N_{0} \quad$ Number density of active sites $\left(\mathrm{crn}^{-2}\right)$ 
no

$\mathbf{n}_{1}$

$n_{2}$

ncu

$\mathbf{n}_{\text {ex }}$

$\mathbf{n}_{\mathbf{i}}$

qnodule

Qtotal

$\mathbf{r}$

ro1

r10

$\mathbf{r}_{12}$

$\mathbf{r}_{21}$

Rel I

t

tol

to2
Refractive index of electrolyte $(=1.34)$

Crmplex refractive index of copper film

Complex refractive index of HOPG (incident $=2.55-1.37 \mathrm{i}$, scattered $:=$ 2.58-1.43i)

Complex refractive index of copper (incident $=1.14 .2 .53 \mathrm{i}$, scattered $=$ 1.08-2.59i)

Complex refractive index of the effective medium

Complex refractive index of species $i$ in the copper film

Charge to form a single nodule (Coulombs/nodule)

Charge density to entire surface (Coulombs/ $\mathrm{cm}^{2}$ )

Radius of nodule $(\mathrm{cm})$

Fresnel reflection coefficient for s-polarized incident light sit the interface between electrolyte and the copper film

Fresnel reflection coefficient for s-polarized scattered light at the interface between th copper film and electrolyte

Fresnel reflection coefficient for a-polarized incident light at the interface between the copper film and graphite

Fresuel reflection coefficient for 8-polarized scattered light; at the interface between graphite and the copper film

Relative Raman intensity

Time (B)

Fresnel transmission coefficient for the incident electric field amplitude through the interface between the electrolyte and the copper film.

(= thare,ine) Fresnel transmission coefficient for incident electric field amplitude through the interface between olectrolyte and graphitu. 
$t_{10}$

$t_{12}$

$t_{20}$

$t_{21}$

Tbare,inc

tbare,ine

tbare,acat

Tarude,ine

tdrude,ine

$T_{\text {offine }}$

$t_{\text {efrinc }}$

$t_{e x t, \text { eat }}$

$t_{\text {m }}$
Fresnel transmission coefficient for s.polarized electric field amplitude of scattered light through the interface between the copper film and the electrolyte.

Fresnel transmission coefficient for s-polarized incident electric field amplitude through the interface between the copper film and the graphite.

(= tbare,scat $_{\text {bresnel }}$ Fransmission coefficient for s-polarized electric field amplitude of scattered light through the interface between graphite and the electrolyte.

Fresnel transmission coefficient for s-polarized electric field amplitude of scattered light through the interface between graphite and the copper film.

$\left(=t_{\text {bare,inc }}\right)^{2}$ Transmittance for incident light intensity in through bare graphite/electrolyte interface

Transmission coefficient for incident electric field amplitude through electrolyte/HOPG interface

Transmission coefficient for scattered electric field amplitude through HOPG,/electrolyte interface

( $\left.=t_{\text {drude,ine }}\right)^{2}$ Transmittance for incident light intensity in through the copper layer accounting for thin film interference effects

Total transmission coefficient through the copper film for incio'ent electric field amplitude, including thin film interference

$\left(=t_{\text {eff,ino }}\right)^{2}$ Effective transmittance for incident light intensity in through the copper layer

Overall effective transmission coefficient for incident electric field axplitude in through the copper film

Overall effective transmission coefficient for scattered electric field amplitude out through the copper film

Time at current maximum 
Volurne of nodule $\left(\mathrm{cm}^{3}\right)$

W Jacobian matrix

x bt $t_{m}$, substitution in the Scharifker and Mostany model

zF Charge per mole of depositing species $(\mathrm{C} / \mathrm{mol})$

$\alpha$

$\alpha$

$\Delta v$

$\phi_{0}$

$\phi_{1}$

$\phi 2$

$\gamma$

hv

hv'

$\lambda_{\text {ine }}$

$\lambda_{\text {seat }}$

$\mu$

$\boldsymbol{\theta}$

$\theta_{\mathbf{i}}$

$\rho$
b/A, substitution in the Scharifker and Mostany modei

Raman scattering coefficient

Raman shift

Light propagation angle in electrolyte

Complex light, propagation angle in copper film

Complex light propagation angle in graphite layer

$\equiv 2$, Screening factor related to symmetry

Incident radiation

Scattered radiation

Wavelength of incident light

Wavelength of scattered light

Dipole moment

Fraction of surface covered by copper

Volume fraction of species $i$ in the copper film

Density of metal deposit $\left(\mathrm{g} / \mathrm{cm}^{3}\right)$ 


\section{Acknowledgements:}

During the time I have spent working on the research for my thesis, I have relied heavily on assistance and encouragement from many people.

I would like to thank my advisor, Rolf Muller, for giving me patient guidance and support; my scientific mentors Dan Schwartz and Mike Armstrong, for getting me started and helping along the way. I would also like to acknowledge my thesis committee members Charles Tobias and James Evans, for their helpful criticisms.

I would like express my special thanks to my parents, Dorothy and Ilhan Kinaci, for their continual love and support and my friends, Dan and Caryn Graves, Reha Bafrali, Rolfe Anderson, Sholeh Hessami, Gerhard Matzen and all the Tobias/Muller research group for stimulating discussions and entertaining diversions. Finally, a huge thank-you goes to my fiance, Bob Crocker, for the wealth of emotional and technical support he has given me.

This work was supported by the Director, Office of Energy Research, Office of Basic Energy Sciences, Materials Sciences Division of the U.S. Department of Energy under contract No. DE-AC03-76SF00098. 


\section{Chapter 1:}

\section{INTRODUCTION AND LITERATURE REVIEW}

\subsection{Introduction:}

Electrodeposition, or electroplating, a process whereby a metal is deposited on a surface from a solution of its ions by means of passing an electric current, can be divided into two processes: nucleation and growth. Nucleation, which dominates very early stages, is a heterogeneous reaction of reducing ions into a small cluster forming a new phase on an electrode. Growth is the process of reducing ions on the existing nuclei, thereby expanding the new phase. The growing nuclei eventually overlap and form a film of the reduced metal on the substrate. The nucleation and growth reactions are important in determining the properties of the resulting film, especially those of thin films with thicknesses on the order of tens of nanometers.

Electrodeposition is a relatively old science, yet how the deposit is initiated is not well understood. There are numerous theories on nucleation, with no clear consensus among researchers studying nucleation phenomena. There are several variables in the system, from the conditions of the surface and the electrolyte to the applied driving force.

This thesis concentrates on electrodepositing metal on non-metal, low electron density substrates. Nucleation is very dependent on the substrate being used ${ }^{1}$. Metal on metal deposition has been widely researched yet there are only a few studies with metal deposition on non-metal substrates. Non-metal substrates exhibit lower nucleation densities than metals, which makes direct counting of the nuclei possible; therefore such substrates are good models for the study of the nucleation process. 
Specifically, in this research highly oriented pyrolytic graphite (HOPG) was investigated as a model low electron density substrate to study the nucleation and growth of copper. HOPG is a logical choice, because it exhibits similar deposition behavior as other non-metal substrates such as conducting polymers and semiconductors, yet it is easier to work with. It is highly crystalline and flat and simple to prepare.

When a metal is electrodeposited on a graphite surface, the resulting deposit appears as seen in the scanning electron micrograph in figure 1-1. In this figure the black background is H.PG and the white shapes are copper nodules deposited on it. The deposit is not a thin continuous film, but instead consists of individual nodules. The nodular deposit can be explained by examining the two reactions that occur during electrodeposition. The first one is nucleation. In this reaction a few atoms of the depositing metal reduce on the HOPG surface and cluster together to form a nucleus of the new phase. After the addition of a critical number of atoms to the nucleus, it becomes stable. The number of atoms in the critical nucleus is dependent on the electrodeposition conditions. The second reaction is the growth of the nucleus which involves metal depositing on itself.

These reactions can have very different rates. If the kinetics of the growth reaction is much faster than that of the nucleation reaction, as soon as a nucleus is formed, further ions will reduce on the nucleus, contributing to the growth of the nucleus rather than starting a nucleus elsewhere. This leads to a few, large nodules rather then many small ones. The nucleation density, the number of nuclei per area, then can be estimated by examining these nodules once they have grown large enough to be visible in an SEM, since we can assume all of the nuclei that formed, resulted in a nodule. 


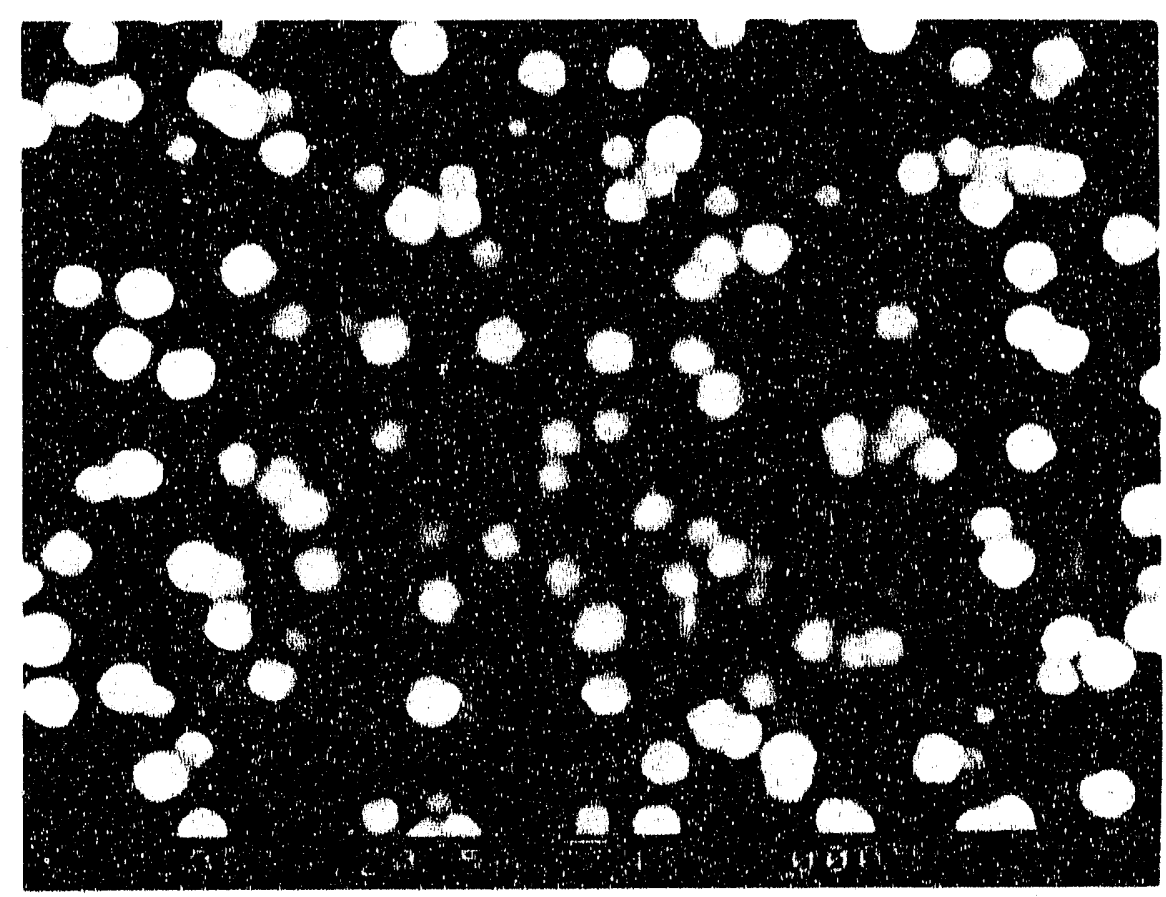

Figure 1-1: Scanning electron micrograph of copper deposit onto HOPG electrode. The dark background is the highly oriented pyrolytic graphite electrode. The light features are the electrodeposited copper nodules.

The main difference between metal on metal deposits versus metal on graphite deposits is the nucleation density. Recently, Armstrong, studied copper deposition on platinum using the scanning tunneling microscope (STM). ${ }^{2}$ In his research, he found nucleation densities on the order of $10^{11}$ nuclei $/ \mathrm{cm}^{2}$. A similar experiment performed on HOPG resulted in nucleation densities on the order of $10^{7}$ nuclei $/ \mathrm{cm}^{2}$.

The importance of nucleation densities can be easily understood following the growth of two deposits: one with high and another with low nucleation density, and comparing the two cases. Where the nodules are close together the continued growth of the nodules cause them to overlap. Eventually, a continuous deposit is generated, which covers the entire surface. Where the nodules are far apart, the 
nodules have to grow much larger before complete coverage is achieved, which results in very thick metal coatings and greater surface irregularity. As summarized in figure 1-2 high nucleation densities lead to thin, continuous films and low nucleation densities result in thick coatings. The representation of the growth of nuclei depicted in figure 1-2 is based on the simple notion of the nucleation density remaining constant during the growth stage. The assumption of constant nucleation density is only valid under certain deposition conditions.

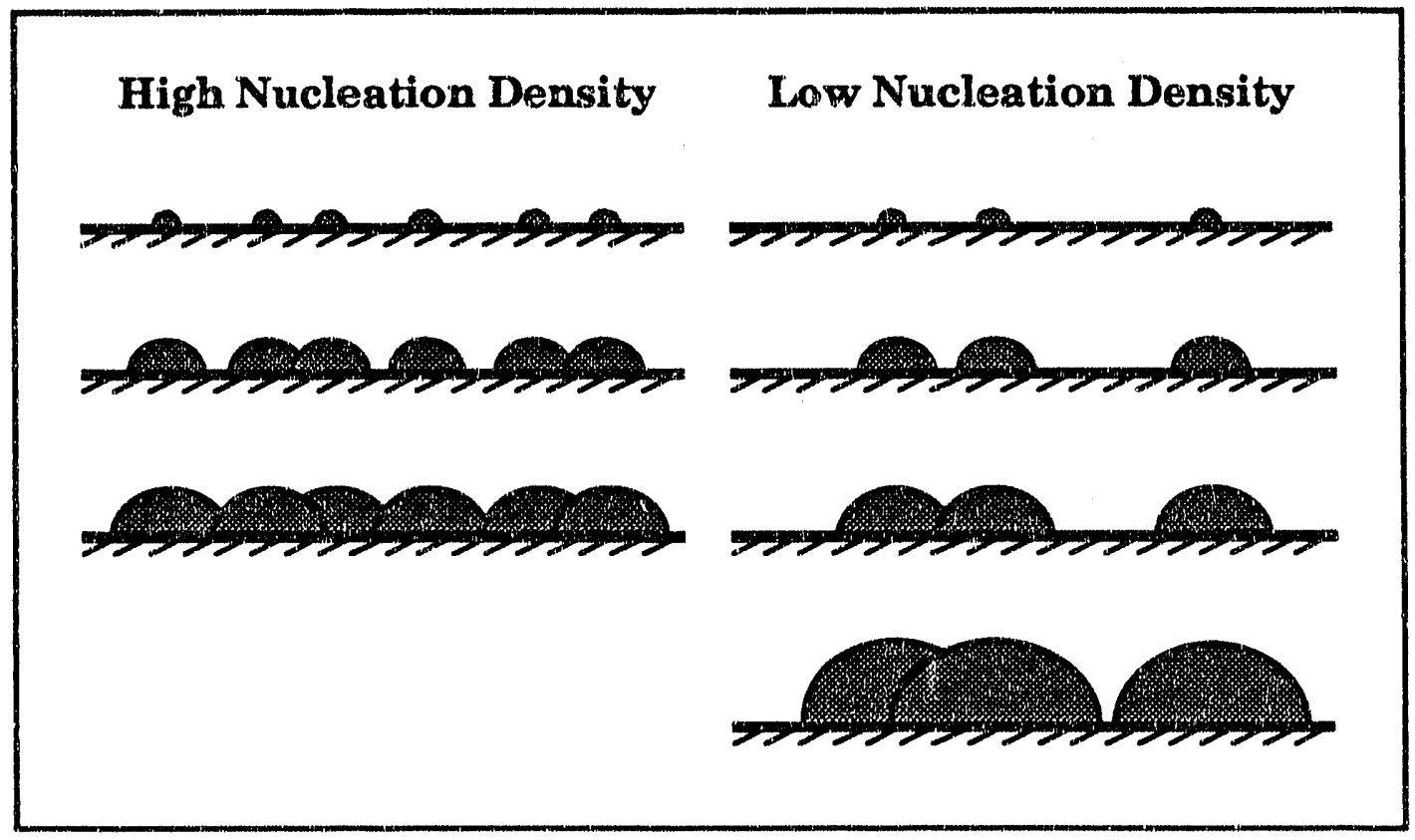

Figure 1-2: Schematic representation of the growth of two metal deposits; one with high and the other with low nucleation density.

The distinction made between instantaneous and progressive nucleation is depicted schematically in figure 1-3 In instantaneous nucleation, all of the nuclei appear at once. The growth of existing nuclei is favored over continued nucleation, therefore no further nuclei appear. The nucleation density is constant and at the end of deposition all of the nodules are of uniform size.

With progressive nucleation the nucleation reaction is lavored over the 
growth of existing nuclei, therefore generation of nuclei continues. The nucleation density increases with time and at the end of the experiment there is a large distribution of nodule sizes.

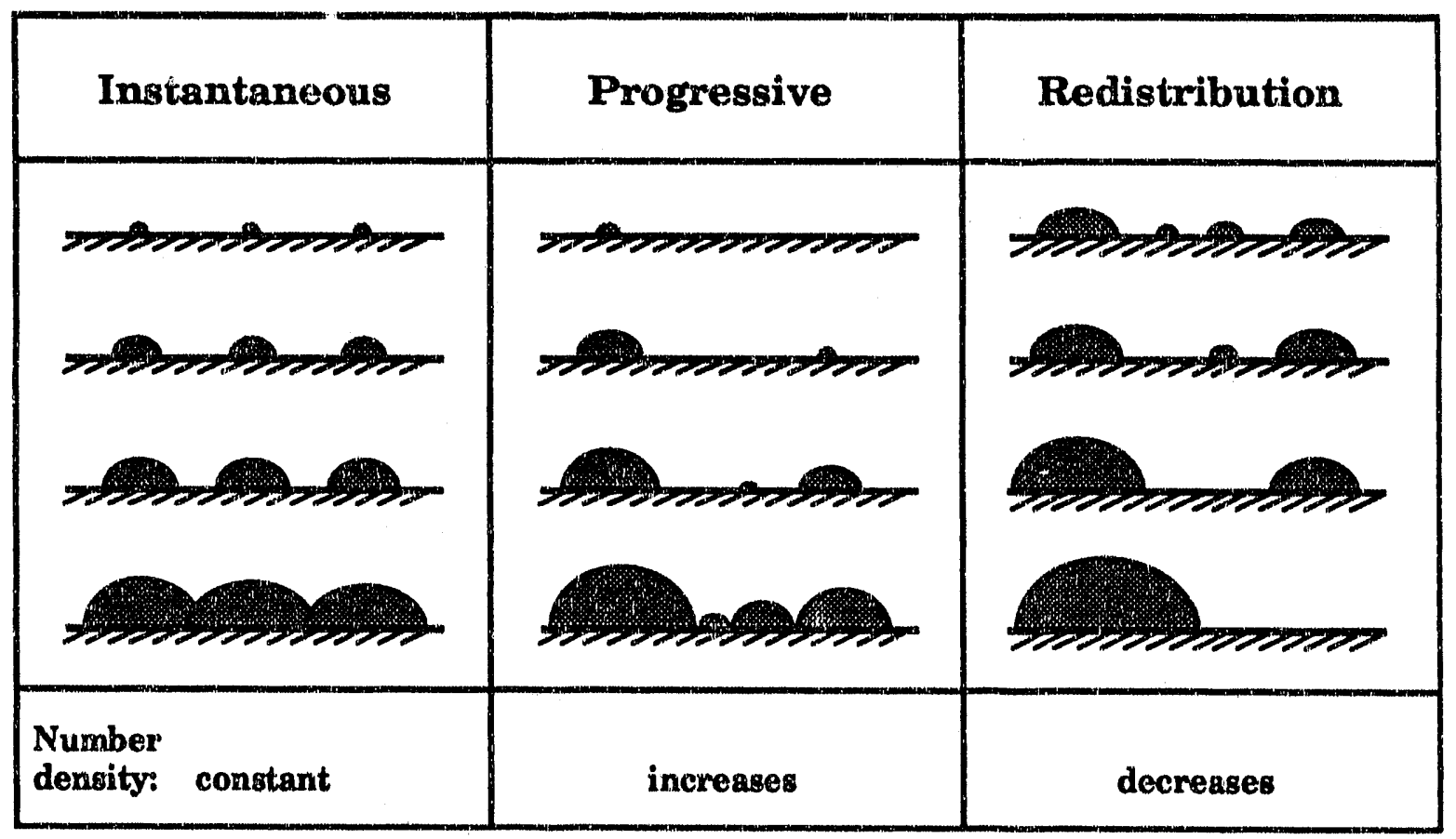

Figure 1-3: Schematic representation of time steps during electrodeposition. The first two columns illustrate the effect of instantaneous and progressive nucleation. The last column illustrates the effect of material redistribution.

An added complication is the possibility of material redistribution. Because there is a large surface energy difference between the metal surface and graphite, larger nodules are more stable than the small ones, creating a driving force for material redistribution. Larger nodules grow at the expense of smaller ones, and the apparent nucleation density decreases during the experiment.

In many of the deposits the nodules appear to follow invisible patterns on the surface. Sorne sites exists on the surface where deposition is favored. Earlier studies have examined the type of structures that cause favored deposition. These sites, referred to as active sites can be: structural imperfections such as steps, dislocations, 
and grain boundaries; lattice defects such as impurities and vacancies; adsorbed species, scratches, cracks or electrode edges. ${ }^{3}$

\section{Applications:}

Quite frequently it is necessary to deposit metal films on non-metallic substrates. These types of interfaces are commonly encountered in the semiconductor industry. Interconnects and micromachines are examples of where one sees metal and semiconductor interfaces.

The original motivation behind this work, however, was exploring ways of making X-ray optics in a simpler and more economic fashion. In X-ray mirrors, there are alternating layers of high and low electron density materials. 4 These layers are continuous and thin, about ten nanometers thick. They are currently produced by evaporation and sputiering techniques which are slow and costly. If these structures could be produced electrochernically, preferably from a single solution, the effort of production and the cost would be greatly reduced. A good candidate for the low electron density material in this application is a conducting polymer such as Polyaniline, which can be electrodeposited directly from the same acid bath as the metal.

One of the obstacles to making these muliilayers has been the trouble with the low nucleation density of metal deposits on the conducting polymer. Methods determined to increase nucleation densities on graphite are expected to be easily applied to the conducting polymer substrates. 


\subsection{Nucleation and Growth Theories:}

Nucleation of a foreign material onto a substrate is assumed to take place on active sites. Electrocrystallization is a specific case of phase formation, therefore the steady state rate of heterogeneous nucleation can be described by the classical theory of nucleation, if the basic kinetic and thermodynamic parameters of the process are expressed in terms of the electrochemical quantities. The results of this treatment are summarized by Vargas and Varma. 5 Other authors have developed atomistic theories of nucleation, which treat nuclei as atom clusters, avoiding the use of continuum properties, such as surface tension. ${ }^{6}$

Several theories exist which account for the time dependence of the number of nuclei. The significant ones are described in the following.

\section{Fleischmann and Thirsk:}

The Fleischmann and Thirsk model ${ }^{7}$ begins with the basic assumption that there are a fixed number of active sites on the surface $\left(\mathrm{N}_{0}\right.$ is a constant independent of potential.) and each has the same probability of being converted to a nucleus. The overall nucleation rate is expressed as a first-order depletion of active sites. This rate is proportional to the number of remaining active sites on the surface. The expression for the time dependent number of nuclei is:

$$
\begin{aligned}
& N(t)=N_{0}[1-\exp (-A(\eta) t)] \\
& \text { where: } t=\text { time (sec) } \\
& \left.N(t)=\text { Number density of nuclei (nuclei/cm }{ }^{2}\right) \\
& A=\text { Nucleation rate per active site (dependent on the } \\
& \quad \text { overpotential) }\left(\sec ^{-1}\right)
\end{aligned}
$$

This model predicts that at long times all of the active sites will be converted to nuclei. 


$$
\lim _{t \rightarrow \infty} N(t)=N_{0}
$$

For the two special cases of fast nucleation on few active sites and slow nucleation on many active sites, equation [1-1] can be simplified. The case with fast nucleation on few sites corresponds to instantaneous nucleation and equation [1-1] becomes:

$$
N(t)=N_{0}
$$

Progressive nucleation occurs when there is slow nucleation on many sites. Equation [1-1] then becomes:

$$
N(t)=N_{0} A t
$$

Many researchers use a modified Fleischmann and Thirsk model, which fits experimental data better. In the modification, the total number of active sites on the surface is allowed to vary with the overpotential. $\left(\mathbb{N}_{0}=f(\eta)\right)$

\section{Kaishev and Mutaftshiev:}

The theory of Kaishev and Mutaftshiev ${ }^{8}$, which was later generalized by Markov and Kashchiev ${ }^{9}$, is based on the assumption that different active sites on the surface may have different critical overpotentials for nucleation.

$$
N(t)=\int_{0}^{\eta} Z\left(\eta_{c}\right)\left[1-\exp \left(-A\left(\eta_{c}\right) t\right)\right] d \eta_{c}
$$

where: $\eta_{c}=$ critical overpotential below which an active site can not be nucleated

$\mathrm{Z}\left(\eta_{\mathrm{c}}\right)=$ density function of active sites having critical overpotential $\eta_{c}$ 
At long times this theory predicts that a "saturation" number of crystals exists at each applied overpotential, $\eta$.

$$
\lim _{t \rightarrow \infty} N(t)=\int_{0}^{\eta} Z\left(\eta_{c}\right) d \eta_{c}=N_{s}(\eta)
$$

where: $N_{s}(\eta)=$ the saturation number of nuclei dependent on overpotential.

\section{Markov and Stoycheva:}

The Markov and Stoycheva model ${ }^{10}$ combines aspects of both the Fleischmann and Thirsk model and the Kaishev and Mutaftshiev model. It is derived from the Kaishev and Mutaftshiev model and the expression has the same form as the modified Fleischmann and Thirsk model.

$$
N(t)=N_{s}(\eta)[1-\exp (-A(\eta) t)]
$$

In this model, the saturation number of active sites, which is dependent on the overpotential, is identical to that described in the Kaishev and Mutaftshiev model. At long times the model predicts the number of nuclei will reach this saturation value.

\section{Fletcher and Lwin:}

The Fletcher and Lwin model ${ }^{11}$ is based on the following assumptions:

i. The total number of active sites is a constant independent of overpotential.

ii. All active sites experience the same overpotential for nucleation.

iii. A random distribution of surface energies exists at the interface between crystal nuclei and active sites. 
The first two assumptions agree with the Fleischmann and Thirsk model.

The third assumption bases the differences between active sites on the differences in the surface energies of the active sites rather than the overpotential, as was assumed in the Kaishev and Mutaftshiev model. The authors further equate the distribution of surface energies to the distribution of activation rates. This treatment distinguishes sites based on the kinetics of nucleation rather than thermodynamic differences. The thermodynamic differences between sites results in the potential dependent saturation number of active sites. The expression for the time dependent number nuclè tassed on the Fletcher and Lwin model is:

$$
N(t)=\int_{0}^{\infty} N_{0}[1-\exp (-A(\eta) t)] \phi(A) d A
$$

$$
\text { where: } \begin{aligned}
\phi(A)= & \text { probability density function of the activation } \\
& \text { rates }(\phi(A) \text { dA is the fraction of active sites having } \\
& \text { rates between } A \text { and }[A+d A])
\end{aligned}
$$

At long times this model predicts all of the active sites will become nucleated. This also agrees with the Fleischmann and Thirsk model. (equation [1-2])

\section{Growth Mechanisms:}

Various mechanisms have been considered for the continued growth of a metal deposit. ${ }^{12}$ These mechanisms are listed below and illustrated in figure 1-4.

A. 1. Diffusion of ionic species through the solution directly to the location of lattice build up

2. Discharge of species at the site where the lattice is to be built up 3. Lattice formation

B. 1. Diffusion of ionic species through the solution to the surface 2. Charge transfer on the surface 
3. Diffusion of discharged species over the surface to sites where the lattice is built up

4. Lattice formation

C. 1. Diffusion of innic species through the solution to the surface

2. Diffusion of charged species over the surface to sites where the lattice is to be built up

4. Charge transfer and lattice formation

Of the listed mechanisms the pathway $\mathrm{C}$ is considered to be the most likely method of lattice growth by Gerischer 13 and by Conway and Bockris. 14k

\subsection{Techniques for Analysis of Nucleation:}

Various methods are used for the analysis of nucleation in metial deposition: ${ }^{5}$

1. Determining the number of nuclei by direct counting of the nuclei fornted during the process.

2. Determining the nucleation density by indirect counting methods such as light scattering.

3. Measuring the growth current, analyzing its transient and applying on of the models to determine the number of nuclei. 


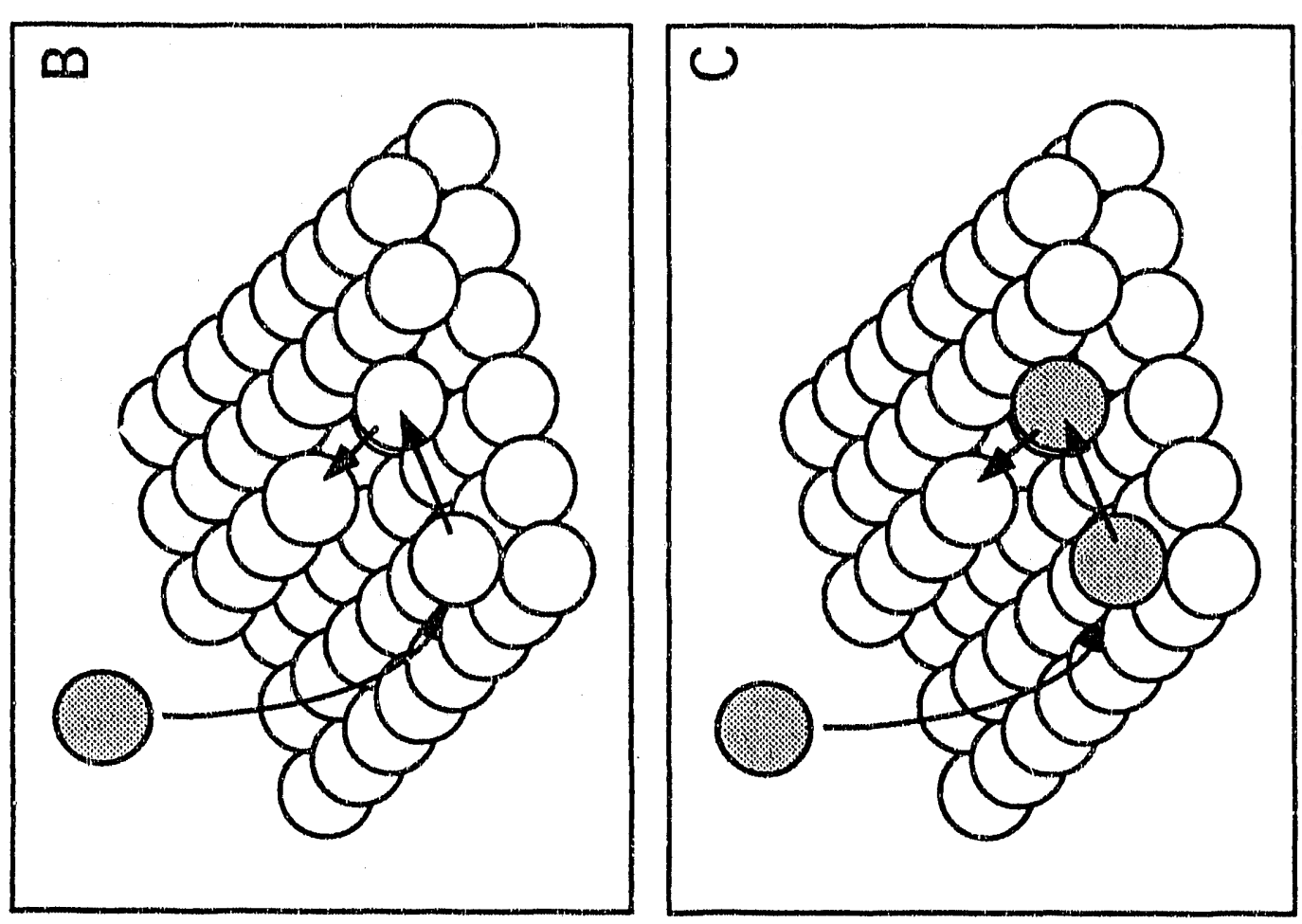

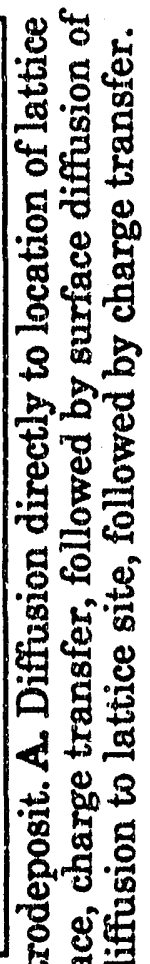
过

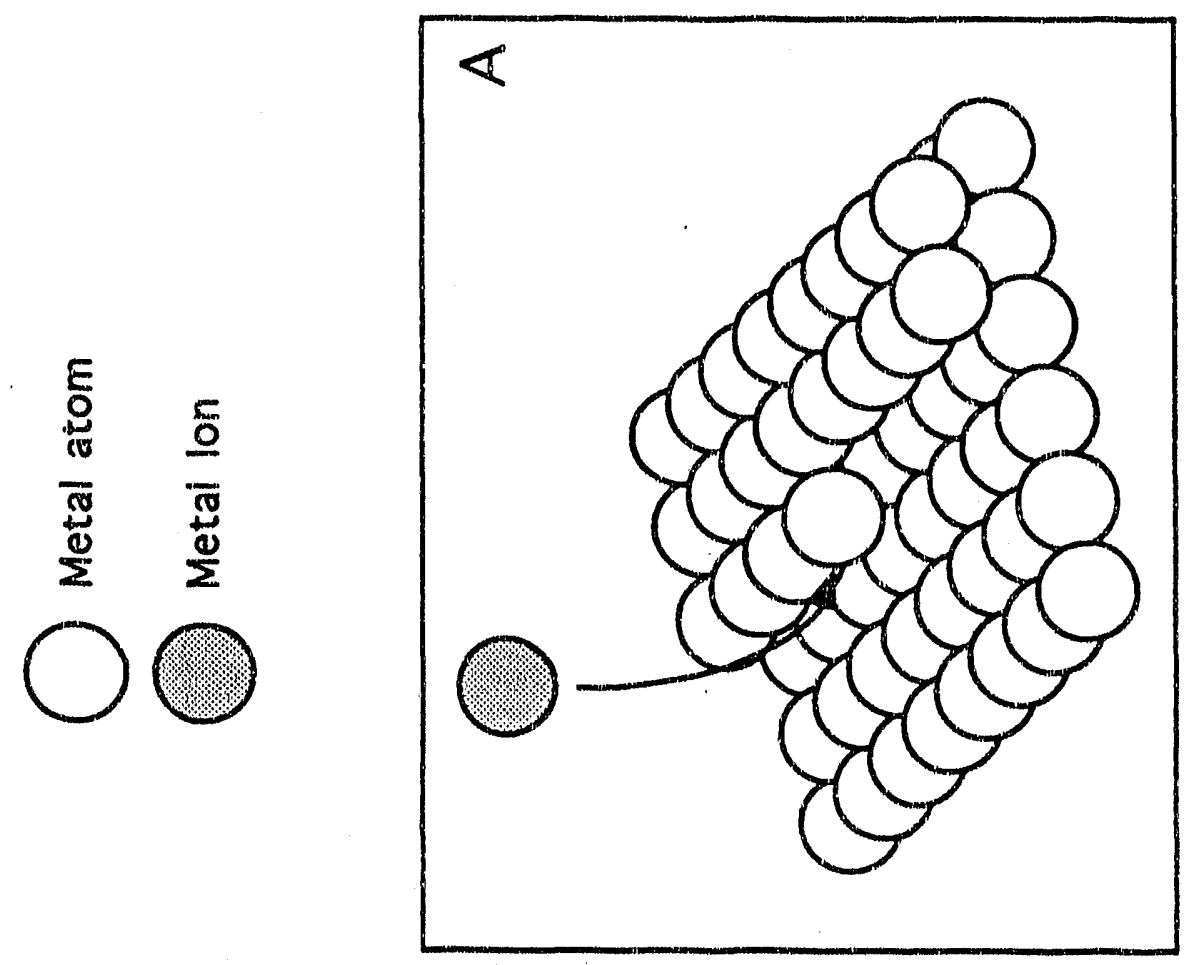

용ำ

曲

本管

它星

웅

声产.

声鬼

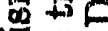

롱

要

무웡

害焉

홍

$p=ㅇ$

분

경ㅇㅇ

猔

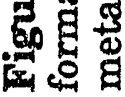


In this study, we have determined nucleation densities by directly counting the nuclei. Determination of nucleation density from the current transient to a potentiostatic step and an indirect counting method were also attempted.

In order to determine the nucleation density from the current transient, an appropriate model for the nucleation is combined with the laws governing the growth of the nuclei. In the initial stages of electrodeposition, the nuclei grow independently form one another. The overall current transient is a superposition of the individual nuclei currents. 12

$$
i(t)=\int_{0}^{t} I(\tau, t) N^{\prime}(\tau) d \tau
$$

$$
\text { where: } \begin{aligned}
& i(t)=\text { overall current density } \\
& \tau=\text { time when nuclei is germinated } \\
& I(\tau, t)=\text { current caused by the growth of an individual } \\
& \text { nucleus germinated at time } \tau \text { and observed at } \\
& \text { time } t
\end{aligned}
$$

Except in concentrated solutions, the growth of the nucleus is controlled by mass transport through the electrolyte. The current to an individual nucleus under mass transport control is determined by the rate of hemispherical diffusion. The current to an individual nucleus has been found to be: ${ }^{15}$ 


$$
I(t)=\frac{z F \pi(2 c D)^{3 / 2}[1-\exp (-z F \eta / R T)]^{3 / 2} M_{w}^{1 / 2} t^{1 / 2}}{\rho^{1 / 2}}
$$

where: $\quad \mathrm{zF}=$ charge per mole of depositing species $(\mathrm{C} / \mathrm{mol})$

$\mathrm{c}=$ Bulk concentration of electroactive species $\left(\mathrm{mol} / \mathrm{cm}^{3}\right)$

$\mathrm{D}=$ Diffusion coefficient of depositing species $\left(\mathrm{cm}^{2} / \mathrm{s}\right)$

$\eta=$ overpotential (V)

$\mathrm{R}=$ universal gas constant

$\mathbf{M}_{\mathbf{w}}=$ molecular weight of deposited metal $(\mathrm{g} / \mathrm{mol})$

$\rho=$ density of deposited metal $\left(\mathrm{g} / \mathrm{cm}^{3}\right)$

While the individual nuclei on the surface are growing independently, the current to the entire surface can be determined by equation [1-9]. The equation for the current density, assuming the Fleischmann and Thirsk model for nucleation and mass transport controlled growth, has been solved. ${ }^{16}$ The solution for the limiting cases of instantaneous and progressive nucleation have also been reported. ${ }^{15}$

For instantaneous nucleation:

$$
I(t)=\frac{N_{0} z F \pi(2 c D)^{3 / 2}[1-\exp (-z F \eta / R T)]^{3 / 2} M_{w}^{1 / 2} t^{1 / 2}}{\rho^{1 / 2}}
$$

For progressive nucleation:

$$
I(t)=\frac{2 \mathrm{AN}_{0} \mathrm{zF} \pi(2 \mathrm{cD})^{3 / 2}[1-\exp (-z F \eta / R T)]^{3 / 2} \mathrm{M}_{\mathrm{w}}^{1 / 2} \mathrm{t}^{3 / 2}}{3 p^{1 / 2}}
$$

The previous expressions for the current density no longer apply when the nuclei do not grow independently of one another. This occurs when the concentration depletion zones surrounding the nuclei start to overlap. In this case, the expression for the growth current of an individual nucleus does not apply. 
Determining the overall current density expression after the diffusion fields overlap, has proven to be a difficult problem to solve. Scharifker et al. ${ }^{17}$ have made simplifying assumptions in order to solve it.

Scharifiker et al. simplify the three-dimensional diffusion problem to a twodimensional one, by projecting the hemispherical diffusion zone around the nuclei onto the electrode surface. This allows them to estimate the hemispherical diffusion to the nuclei by linear diffusion to the circular projected areas of the hemispheres. They apply the Avrami theorem to estimate the amount of overlap of these circular areas. The resulting expression for the overall current density with instantaneous nucleation is:

$$
\begin{gathered}
i(t)=\operatorname{zFc}\left(\frac{D}{\pi t}\right)^{1 / 2}\left[1-\exp \left(-N_{0} \pi k D t\right)\right] \\
\text { where: } \quad k=\left(8 \pi c M_{w} / \rho\right)^{1 / 2}
\end{gathered}
$$

And with progressive nucleation:

$$
\begin{aligned}
& i(t)=\operatorname{zFc}\left(\frac{D}{\pi t}\right)^{1 / 2}\left[1-\exp \left(\frac{-\mathrm{AN}_{0} \pi k D t^{2}}{2}\right)\right] \\
& \text { where: } \quad k=\frac{4}{3}\left(8 \pi c M_{w} / \rho\right)^{1 / 2}
\end{aligned}
$$

Scharifker and Mostany 18 later improved the above treatment to develop a general expression for the current transient, so as to calculate the number density of active sites and the nucleation rates per site directly from measured current transients. Their expression for the current transient is: 


$$
i(t)=\operatorname{zFc}\left(\frac{D}{\pi t}\right)^{1 / 2}\left\{1-\exp \left[-N_{0} \pi k D\left(t-\frac{[1-\exp (-A t)]}{A}\right)\right]\right\}
$$

where: $k$ is defined by equation [1-19]

A more detailed discussion on how to determine the nucleation density from the current transient using the Scharifker-Mostany model is included in section 2.3 of this thesis.

Other significant studies include: the Fleischmann et al. ${ }^{12}$ solution of the current transient expression after the overlapping of the growth centers occurs. Recently, Abyaneh and Fleischmann ${ }^{19}$ have formulated current-time transients for deposition under charge-transfer control, as well as diffusion control. Experimental study of the nucleation phenomenon have been reported by many researchers. Milchev et al. have examined mercury ${ }^{20}$ and silver ${ }^{21}$ nucleation on platinum. Scharifker et al. ${ }^{22}$ have examined the electrodeposition of mercury on vitreous carbon. Fletcher and Deutscher ${ }^{23}$ have studied nucleation of lead on carbon microelectrode assemblies.

\subsection{Properties of Highly Oriented Pyrolytic Graphite:}

Highly oriented pyrolytic graphite (HOPG), is produced from pyrolytic graphite (PG), which is heated to elevated temperatures $(\approx 3400 \mathrm{C})$ while stress is applied ${ }^{24}$. This procedure enhances the crystallite alignment. HOPG finds its main application in X-ray monochromators.

The ideal graphite crystal consists of layers of carbon atoms arranged in hexagonal rings that are stacked in a sequence ABAB... (Figure 1-5) The carbons in the layers are connected by stable covalent bonds, but the connection between layers 
is by weak van der Waals bonds. The carbon-carbon bond length in the hexagonal rings of the layers is $1.42 \AA$. The distance between atoms in the direction of the c-axis, which is perpendicular to the rings, is $3.35 \AA$.

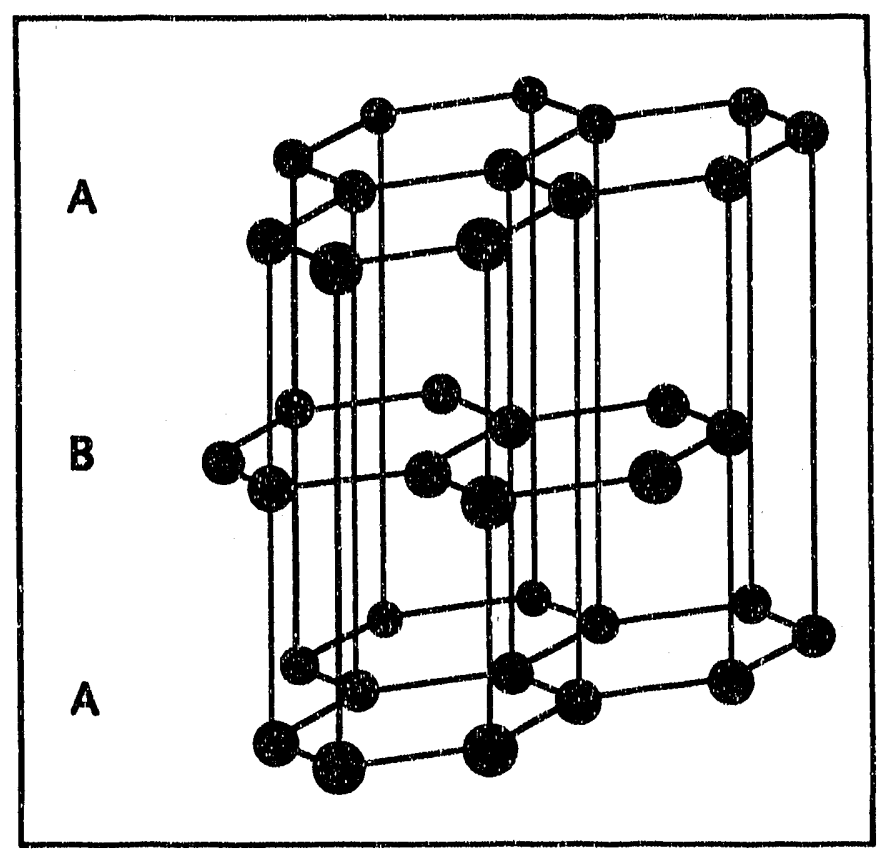

Figure 1-5: Three planes consisting of hexagonal rings in the crystal structure of graphite are illustrated. Atomic distances within the rings is $1.42 \AA$, while the atomic distances between layers is $3.35 \AA$. Only every other atom in the hexagonal grid has an atom directly above and below it.

A freshly cleaved surface of HOPG is prepared by contacting a piece of adhesive tape to the graphite surface and then removing a thin layer of graphite with the tape. The cleaved surface is smooth and highly reflective. The cleavage occurs along the basal plane of the graphite, paraliel to the direction of the hexagonal rings. The optical properties of graphite have been determined by Taft and Philipp. ${ }^{25}$

HOPG has anisotropic properties between the direction of the $c$-axis and the dir"sition parallel to the hexagonal rings. The electrical resistivity, for instance, is about $10^{-2} \Omega$-cm in the direction of the c-axis and $4 \times 10^{-5} \Omega-\mathrm{cm}$ along the basal 
plane. ${ }^{24}$ There also are differences in electrochemical responses between different surfaces of graphite. The reduction of oxygen proceeds at much slower rates on the cleaved, basal plane of HOPG, than on the edge plane. ${ }^{26}$ The differential capacitance of the basal plane determined by a-c impedance in aqueous solution is much lower than the capacitance of the edge plane. 27 This type of behavior has been found to be true in general. The electrochemical activity of the basal plane appears to be lower than that of the edge plane orientation. An extended review of the electrochemical properties of graphite and other carbons has been published recently by Kinoshita. 24

The electrode activity of the basal plane is found to increase with certain treatments such as: mechanical polishing, electrochemical treatment, chemical treatment, or thermal treatment. The possible mechanisms are ${ }^{24}$ : a. removal of contaminants from the surface, $b$. the increase of the concentration of surface functional groups that may act as electron transfer mediators, c. the increase of surface area by increasing roughness, or d. the exposure of fresh edge planes or defects that may be sites for electron transfer.

The activity of the carbon electrodes is commonly measured with the $\mathrm{Fe}(\mathrm{CN})_{6}^{3-} \Leftrightarrow \mathrm{Fe}(\mathrm{CN})_{6}^{4-}$ redox reaction. On the basal plane of HOPG, after electrochemical oxidation at $1.5 \mathrm{~V}$ (versus SCE) for 15 minutes, the reduction rate of $\mathrm{Fe}(\mathrm{CN}){ }_{6}^{3-}$ has been found to increase. ${ }^{28}$ Defects generated on the surface have been shown to be the major cause for the increased activity of HOPG basal planes by Robinson et al. ${ }^{29}$ in their STM study of HOPG. McCreery et al. ${ }^{30}$ have found the Raman spectrum of the basal plane of HOPG appears more like the spectrum from the edge plane after modification by anodization and pulsed laser irradiation.

The measurement of preferential adsorption of organic molecules on certain sites on the graphite surface allows determination of the number of such sites. ${ }^{31}$ 
The study of the amount of surface area in the basal planes is accomplished by the adsorption of alkane molecules, which have a strong affinity for the basal planes of graphite. One such study ${ }^{31}$ utilized n-dotriacontane, whose adsorption isotherm approaches the limiting value associated with monolayer coverage. One molecule of n-dotriacontane is calculated to occupy $178 \AA^{2}$ of the basal plane.

The strong adsorption of polar solutes at the edge sites $^{31}$ in non-polar solvents provides a measure of the surface area associated with these sites. In one such study n-butanol was adsorbed onto graphite. One molecule of n-butanol occupies an area the size of $27 \AA^{2}$. Adsorption of water molecules onto the same sites can cause some error in the measurements.

The high degree of crystallinity and the localized electrons in HOPG, makes HOPG an ideal surface for scanning tunneling microscopy (STM). HOPG was one of the first surfaces that was imaged to atomic resolution using the STM. Atomic images have been obtained from scanning in ultra high vacuum, ${ }^{32}$ air, ${ }^{33}$ and water. ${ }^{34}$ A few researchers have studied the electrodeposition of various materials on graphite using the STM, to further understand the nucleation phenomenon. ${ }^{35,36,37,38}$ Poor adhesion of most electrodeposits to the graphite substrate, however, has restricted the application of this method to the study of nucleation. 


\section{References:}

1 A. Milchev, "Role of the Substrate in Electrochemical Nucleation", Electrochim. Acta 28, 947 (1983).

2 M. J. Armstrong, "The Role of Inhibitors During Electrodeposition of Thin Metallic Films", Ph.D. Dissertation, University of California, Lawrence Berkeley Laboratory, LBL-28972 (1990).

3 R. L. Deutscher and S. Fletcher, "Nucleation on active sites: Part V. The Theory of Nucleation Rate Dispersion", J. Electroanal. Chem. 277, 1 (1990).

4 T.W. Barbee Jr., "Multilayers for X-Ray Optics", in Proceedings of SPIE - the International Society of Optical Engineering 563, 2(1985).

5 T. Vargas, R. Varma, "Techniques for Nucleation Analysis in Metal Electrodeposition", in Techniques for Characterization of Electrodes and Electrochemical Processes, p. 717-760, edited by R. Varma and J.R. Selman, Wiley, New York, 1991.

5 D. Walton, "Nucleation of Vapor Deposits", J. Chem. Phys. 37, 2182 (1962).

7 M. Fleischmann, H.R. Thirsk, "Anodic Electrocrystallization", Electrochim. Acta 2, 22 (1960).

8 R. Kaishev, B Mutaftshiev, "Uber die Elektrolytische Keimbildung des Quecksilbers”, Electrochim. Acta 10, 643 (1965).

9 I. Markov, D. Kashchiev, "Nucleation on Active Centres, I. General Theory", J. Crystal Growth 16, 170 (1972).

10 I. Markov, E. Stoycheva, "Saturation Nucleus Density in the Electrodeposition of Metals onto Inert Electrodes, II. Experimental", Thin Solid Films 35, 21 (1976).

11 S. Fletcher, T. Lwin, "A General Probabilistic Model of Electrochemical Nucleation", Electrochim. Acta 28, 237 (1983). 
M. Fleischmann, H.R. Thirsk, "Metal Deposition and Electrocrystallization", in Advances in Electrochemistry and Electrochemical Engineering, Vol. 3, p.123-210, edited by P. Delahay and C.W. Tobias, Wiley, New York, 1963.

13 H. Gerischer, "Mechanism of Electrolytic Deposition and Solution of Metal", Z. Elektrochem. 62, 256 (1958).

14 B.E. Conway, J.O'M. Bockris, "Calculation of Potential Energy Profile Diagrams for Processes in Electrolytic Metal Deposition", Elec rochim. Acta 3, 340 (1961).

15 G. J. Hills, D.J. Schiffrin, J. Thompson, "Electrochemical Nucleation from Molten Salts, I. Diffusion Controlled Electrodeposition of Silver from Alkali Molten Nitrates”, Electrochim. Acta 19, 657 (1974).

16 M. Sluyters-Rehbach, J.H.O.J. Wijenberg, E. Bosco, J.H. Sluyters, “The Theory of Chronoamperometry for the Investigation of Electrocrystallization, Mathematical Description and Analysis in the Case of Diffusion-Controlled Growth", J. Electroanal. Chem. 236, 1 (1987).

17 B. Scharifker, G. Hills, "Theoretical and Experimental Studies of Multiple Nucleation", Electrochim. Acta 28, 879 (1983).

18 B.R. Scharifker, J. Mostany, "Three-Dimensional Nucleation with Diffusion Controlled Growth, Part I. Number Density of Active Sites and Nucleation Rates per Site”, J. Electroanal. Chem. 177, 13 (1984).

19 M.Y. Abyaneh, M. Fleischmann, "General Models for Surface Nucleation and Three-Dimensional Growth: the Effect of Concurrent Redox Reactions and of Diffusion", J. Electrochem. Soc. 138, 2491 (1991).

20 V. Tsakova, A. Milcher, "Comparative Studies of Electrochemical Phase Formation by Amperometric and Microscopic Methods: Part I. Nucleation Kinetics in Dilute Solutions of Mercury Nitrate", J. Electroanal. Chem. 235, 237 (1987). 
21 A. Milchev, T. Chierchie, K. Jüttner, W.J. Lorenz, "Substrate Transformation Phenomena During Electrochemical Nucleation of Silver on Polyfaced Platinum Single Crystal Electrodes: I. Temperature and Overpotential Dependence of the Stationary Nucleation Rate", Electrochim. Acta 32, 1039 (1987).

A. Milchev, T. Chierchie, K. Jüttner, W.J. Lorenz, "Substrate Transformation Phenomena During the Nucleation of Silver on Polyfaced Platinum Single Crystal Electrodes: II. Characterization of the Platinum Surface”, Electrochim. Acta 32, 1043 (1987).

22 G. Gunawardena, G. Hills, I. Montenegro, B. Scharifker, "Electrochemical Nucleation: Part III. The Electrodeposition of Mercury on Vitreous Carbon”, J. Electroanal. Chem. 138, 255 (1982).

23 R.L. Deutscher, S. Fletcher, "Nucleation on Active Sites: Part IV. Invention of an Electronic Method of Counting the Number of Crystals as a Function of Time: and the Discovery of Nucleation Rate Dispersion", J. Electroanal. Chem. 239, 17 (1988).

24 K. Kinoshita, in Carbon: Electrochemical and Physicochemical Properties, Wiley, New York, 1988.

25 E.A. Taft, H.R. Phillipp, "Optical Properties of Graphite", Physical Review 1A 138, A197 (1965).

26 I. Morcos, E. Yeager, "Kinetic Studies of the Oxygen-Peroxide Couple on Pyrolytic Graphite", Electrochim. Acta 15, 953 (1970).

27 J.P. Randin, E. Yeager, "Differential Capacitance Study of Stress-Annealed Pyrolytic Graphite Electrodes”, J. Electrochem. Soc. 118, 711 (1971).

28 R.M. Wightman, M.R. Deakin, P.M. Kovach, W.G. Kuhr, K.J. Stutts, "Methods to Improve Electrochemical Reversibility of Carbon Electrodes", J. Electrochem. Soc. 131, 1578 (1984).

29 R.S. Robinson, K. Sternitzke, M.T. McDermott, R.L. McCreery, "Morphology 
and Electrochemical Effects of Defiss on Highly Oriented Pyrolytic Graphite”, J. Electrochem. Soc. 138, 2412 (1991).

30 R.J. Bowling, R.T. Packard, R.L. McCreery, "Activation of Highly Oriented Pyrolytic Graphite for Heterogeneous Electron Transfer: Relationship between Electrochemical Performance and Carbon Microstructure”, J. Am. Chem. Soc. 111, 1217 (1989).

31 A.J. Groszek in Proceedings of International Symposium on Surface Area Determination, p. 313, edited by D.H. Everett and R.H. Ottewill, Butterworths, London, 1969.

32 G. Binnig; H. Fuchs, Ch. Gerber, H. Rohrer, E. Stoll, E. Tosatti, “Energy Dependent State-Density Corrugation of a Graphite Surface as seen by Scanning Tunneling Microscopy”, Europhysics Lett. 1, 31 (1986).

33 S.I. Park, C.F. Quate, "Tunneling Microscopy of Graphite in Air", Appl. Phys. Lett. 48, 112 (1986).

34 R. Sonnenfeld, P.K. Hansma, "Atomic-Resolution Microscopy in Water", Science 232, 211 (1986).

35 E. Ganz, K. Sattler, J. Clarke, "Scanning Tunneling Microscopy of $\mathrm{Cu}, \mathrm{Ag}$, $\mathrm{Au}$, and Al Adatoms, Small Clusters, and Islands on Graphiten, Surface Science 219, 33 (1989).

36 M. Szklarczyk, J.O’M. Bockris, "In-Situ STM Studies of Lead Electrodeposition on Graphite Substrate”, J. Electrochem. Soc. 137, 452 (1990).

37 M.P. Everson, J.H. Helms, "A Scanning Tunneling Microscope Study of the Electrochemical Polymerization of Perchlorate-doped Polypyrrole on Highly Oriented Pyrolytic Graphite”, Synthetic Metal, 40, 97 (1991).

38 S. Eppell, G. Chottiner, D. Scherson, G. Pruett, "STM of Platinum Deposits on the Basal Plane of HOPG”, Langmuir 6, 1316 (1990). 


\section{Chapter 2:}

\section{DETERMINATION OF NUCLEATION DENSITY}

\subsection{General Experimental Setup:}

\section{Electrochemical Cell:}

All electrochemical cells employed in this study have the same rnain components, though they vary in size. (Figure 2-1) The electrolyte was $1 \mathrm{M}$ aqueous sulfuric acid with various concentrations of copper sulfate. In some experiments small concentrations of chemical additives were also included to study the effect they have on the electrodeposit. The working electrode was ZYH grade highly oriented pyrolytic graphite (HOPG) supplied by Union Carbide Corporation. Immediately before each experiment, the HOPG electrodes were cleaved by sticking adhesive tape on the surface and then removing it. The top few layers of graphite lift off with the tape, leaving a clean surface behind. The electrodes were placed horizontally, facing up towards the counter electrode in all but the Raman experiments, where they were placed vertically, facing the counter electrode. The reaction on the surface is the reduction of cupric ions $\left(\mathrm{Cu}^{++}\right)$to copper metal.

The reference electrode used to measure or set the potential of the working electrode was a $99.999 \%$ pure copper wire in equilibrium with copper ions in the electrolyte.

The counter electrode employed was usually platinum wire mesh, except where copper wire was used. The reaction at this electrode was the oxidation of water. 


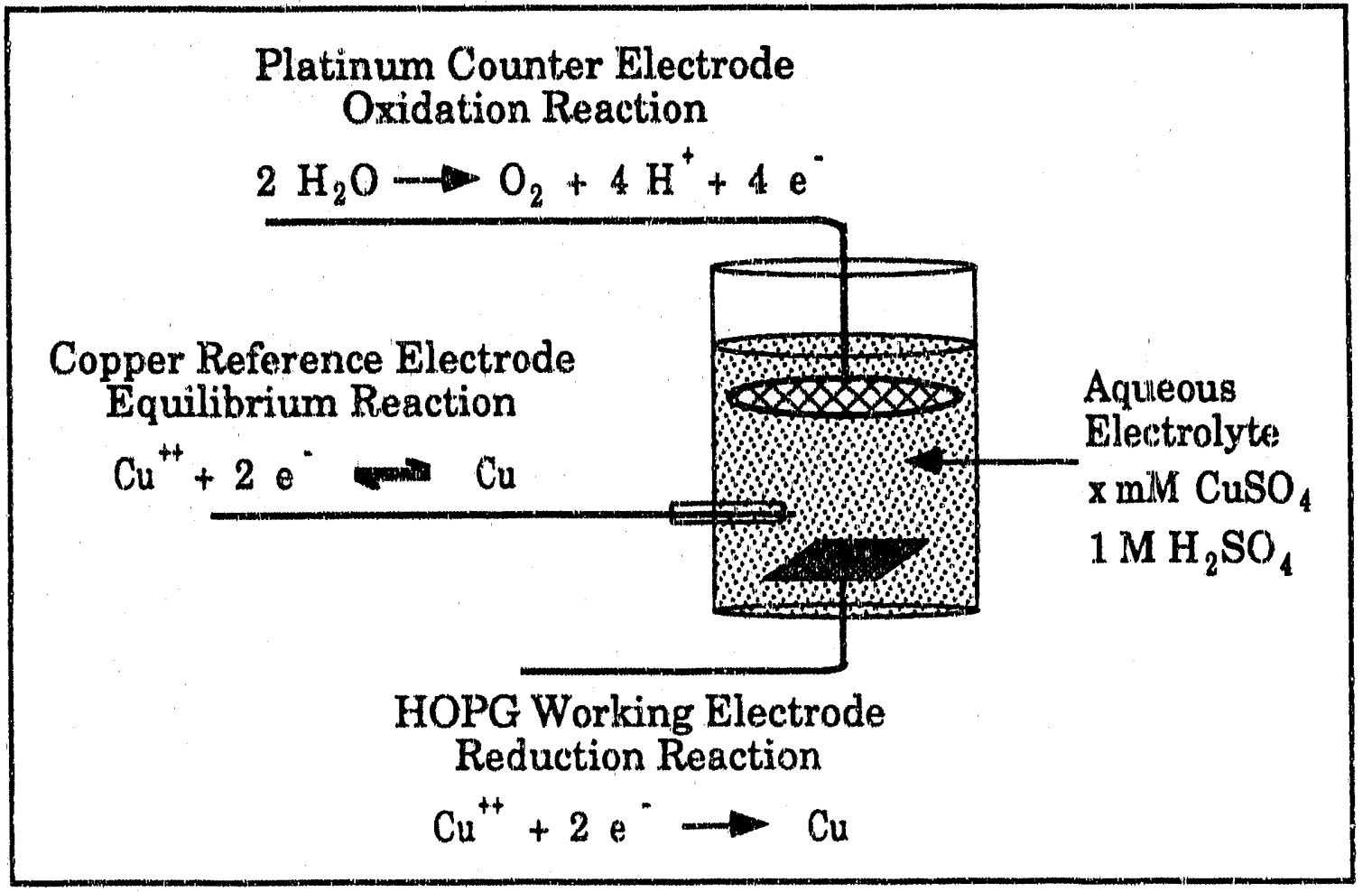

Figure 2-1: Three-electrode electrochemical cell. The electrode materials and the electrode reactions are shown along with the electrolyte concentration. Various concentrations of copper sulfate were used.

\section{Data Collection Equipment:}

To contral the current or the potential in electrochemical experiments, EG\&G Princeton Applied Research Model 173 and Model 273 Potentiostat/Galvanostat were employed. When using the Model 173 Potentiostat (figure 2-2), it was interfaced to an EGRG PAR Model 175 Universal Programmer, to set the desired waveform. The charge passed was monitored using a Princeton Applied Research Model 379 Digital Coul ometer. The current or potential transient wass plotted on a Hewlett-Packard 7644B X-Y Recorder. For experiments, where the speed of the X-Y recordex was not fast enough to track the transient, a Kikusui Electronics COS 5020-ST Storage Oscilloscope was used. A photograph of the transient was 
then taken from the oscilloscope screen for future reference. The camera used was a Polaroid Shackman 7000.

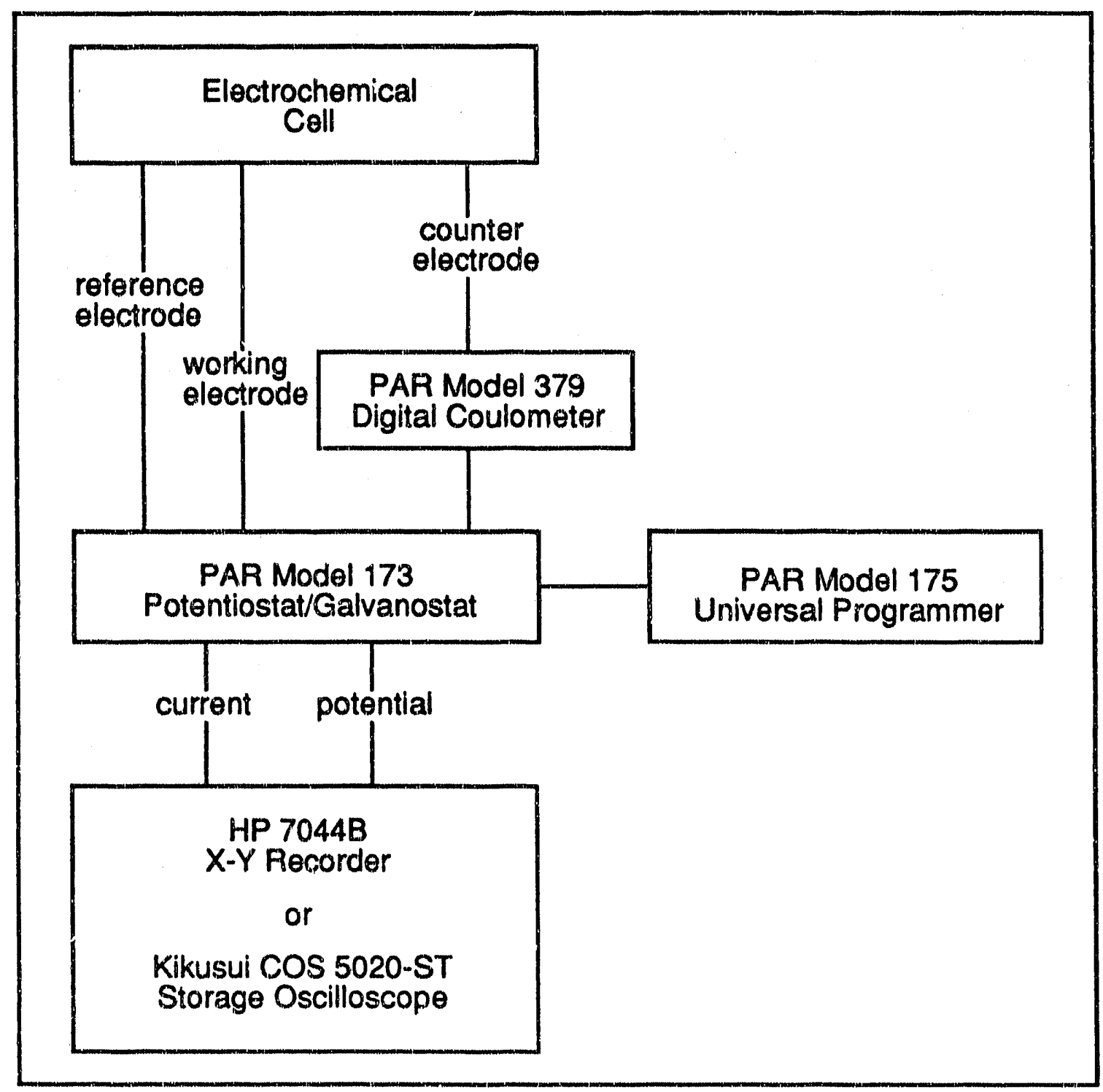

Figure 2-2: Equipment set-up when using model 173 potentiostat.

When the Model 273 Potentiostat (figure 2-3) was used, it was interfaced with an AST Research Model 286 perBonal computer for data acquisition. The software program HEADSTART, which is provided by Princeton Applied Research along with 
the potentiostat, aided in programming the instrument to set the desired waveform and storing of data, such as current, potential and charge.

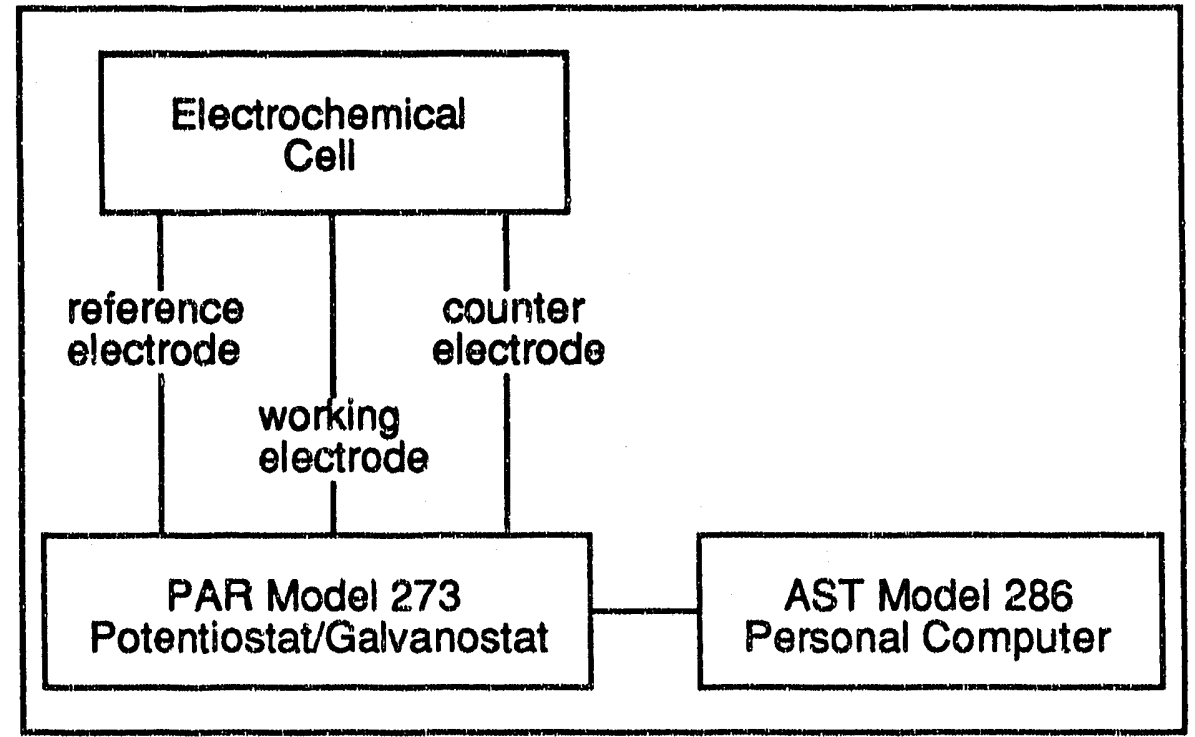

Figure 2-3: Equipment set-up when using model 273 potentiostat.

Because the amount of metal on the surface is directly linked to the charge passed by Faraday's law, monitoring the charge allows for very close control of the amount of metal deposited on the surface, assuming all of the charge passed is consumed by the metal deposition reaction and no side reactions occur.

\section{Surface Analysis Techniques:}

The electrode surfaces were examined using both ex-situ and in-situ techniques to determine nucleation densities and the topography of the electrodeposit. For ex-situ analysis, a scanning electron microscope (SEM) was used. The SEM employed in tis study was an International Scientific Instruments, WB-6. It has the capability of generating large depth-of field images with 40 Angstrom resolution. It is equipped with a Kevex energy dispersive X-ray analyzer (EDAX), which detects X-rays generated by the elements present in the sample. All elements heavier than fluorine are simultaneously determined on the sample with $1 \mathrm{\mu m}$ 
resolution. Two dimensional maps can be produced which indicate the spatial distribution of each element in the sample.

Scanning tunneling microscopy (STM) and Raman spectroscopy were also applied. The STM used in this study was a Digital Instruments, Nanoscope I, employing a scanning head with $0.6 \mu \mathrm{m}$ range. The horizontal positioning of the STM probe and the vertical height data collection are under computer control, with software written by Armstrong. 1 The data acquisition program generates a data array of height or tunneling current over the area examined, with vaxiable sizes for the scan area. More detailed description of the instrument and the software are by Armstrong. 1

Raman experiments were used for determining the copper coverage of the graphite surface. The light source was a one Watt argon ion laser at $488 \mathrm{~nm}$ wavelength. A 1024-channel Princeton Applied Research model 1420 intensified photodiode array was employed for scattered light detection. The detector was controlled using an optical multichannel analyzer unit (OMA III) from Princeton Applied Research. More detailed description of the Raman spectrometer is in Mayer.2

\subsection{SEM Analysis}

After copper is deposited on the HOPG surface, the samples can be analyzed at high magnifications with the SEM. An example of an image from the SEM is pictured in figure 2-4. The dark background is the HOPG substrate and the light features are the copper nodules. The nodules do not appear to be spherical but instead show edges and corners indicating their crystalline nature. We have assumed that the nodule density is the same as the nucleation density, as discussed in section 1.2 . 


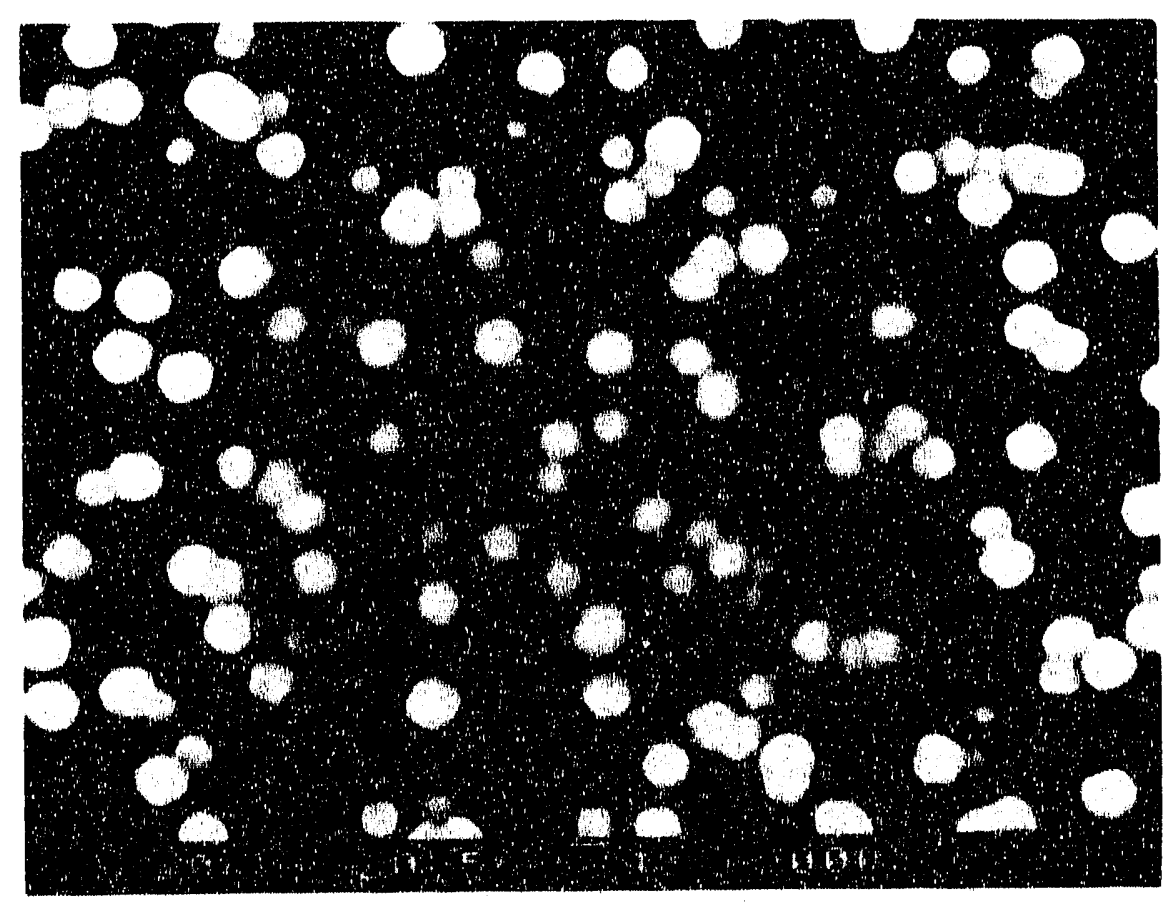

Figure 2-4: SEM of electrodeposited copper on HOPG electrode. The dark background is the graphite and the light features are the copper nodules. Copper deposited onto graphite by a potentiostatic step to $-700 \mathrm{mV}$ for 5 seconds in aqueous electrolyte containing $50 \mathrm{mM} \mathrm{CuSO}_{4}$ and $1 \mathrm{M} \mathrm{H}_{2} \mathrm{SO}_{4}$. Total charge density is $58 \mathrm{mC} / \mathrm{cm}^{2}$.

The nodule number densities are determined by photographing the surface and later counting the number of nodules present. Because automated counting techriques failed to detect nodules that were touching as independent nodules, the counting was done visually. The number densities determined using automated techniques were consistently twenty percent lower than those determined by counting. To get an accurate representation of the nucleation density over the entire surface, the number densities were counted in at least 15 locations over the sample surface. These values were then averaged and their standard deviations determined. 


\section{Experimental Reproducibility:}

Some difficulties were encountered with the reproducibility of nucleation density data when working with HOPG as an electrode. These are caused by the differences in electrochemical activation between the basal and defect sites. Because of this great difference, the defect sites are much favored for metal deposition. At low overpotentials a uniform distribution of nodules was not possible. All the deposited metal tended towards the defects on the surface, such as the step sites. In these experiments, no meaningful data on nucleation density could be collected.

An example of a deposit prepared at low overpotentials is depicted in figure 2-5. This sample was obtained in $50 \mathrm{mM}$ copper sulfate and $1 \mathrm{M}$ aqueous sulfuric acid. The initial potential step was to $-300 \mathrm{rnV}$ (versus copper reference electrode) for one second and the growth was then continued for approximately one hour at $-100 \mathrm{mV}$. The total charge passed was $32 \mathrm{mC} / \mathrm{cm}^{2}$, which corresponds to an equivalent uniform film thickness of $12 \mathrm{~nm}$. Instead of a uniform film, a nodular deposit, with nodules of about 1 to $2 \mu \mathrm{m}$ in diameter, resulted. The nodules are obviously aligned along a few defect lines on the surface. EDAX analysis indicates that all the copper was deposited in these locations, because no copper was detected on the rest of the basal plane.

Early experiments, at various overpotentials, resulted in copper decorating the defect sites on graphite up to about $-600 \mathrm{mV}$ of overpotential. At $-700 \mathrm{mV}$, uniform distribution of copper nodules could be achieved. 'Therefore, nearly all the experiments reported in this thesis were carried out with a minimum of $-700 \mathrm{mV}$ overpotential. 


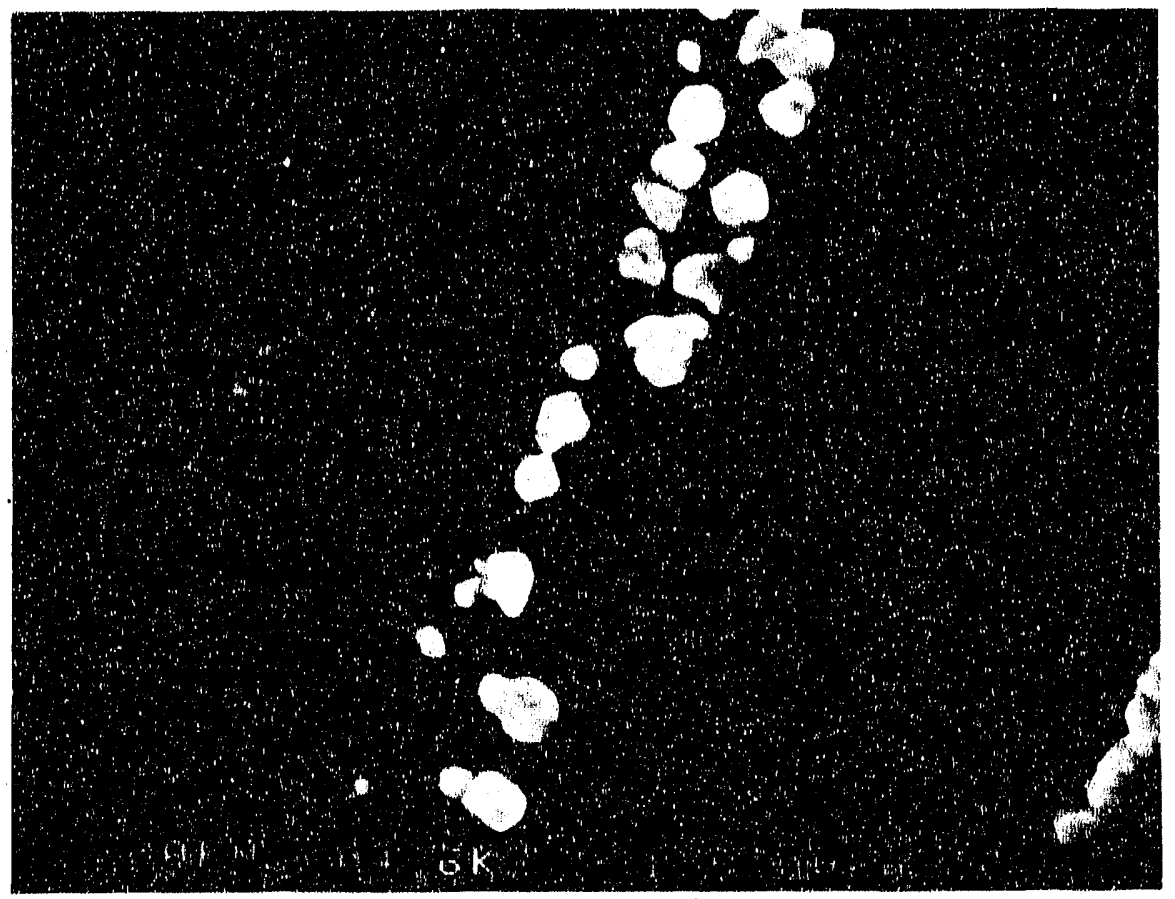

Figure 2-5: Copper deposit along a defect site on the graphite electrode. Copper was deposited from $50 \mathrm{mM} \mathrm{CuSO} 4$ and $1 \mathrm{M}$ aqueous $\mathrm{H}_{2} \mathrm{SO}_{4}$ electrolyte, with a $-300 \mathrm{mV}$ potential pulse for 1 second followed by growth at $-100 \mathrm{mV}$ for $\approx 1$ hour. Charge density $=32 \mathrm{mC} / \mathrm{cm}^{2}$.

Careful sample preparation is the single most crucial step to assure reproducible results. With graphite, many surface defects are likely introduced in the cleaving process. When the surface is cleaved, many layers of graphite are lifted together. Ideally, the cleavage occurs between two atomic layers of graphite, and no steps or vacancies are introduced to the sample. Practically however, only the macroscopic imperfections can be minimized, even when extreme care is taken while cleaving to obtain a visually uniform surface. It is nearly impossible to control the defects on the microscale. Not only are the sample to sample variations large, but different locations on a sample can behave differently, as well. 
In this research, the nucleation densities were determined at a minimum of 15 locations on every sample, and averaged, to minimize the effect these variations have on the results. A solution for the sample to sample variation, however, was not found. Some of the differences between samples are believed to be the result of manufacturing variations, which can not be corrected with cleaving. Where single electrodes were identified as having consistently different electrochemical activities from the average, they were no longer used.

The scatter in the experimental findings must be kept in mind when evaluating the results from this study. In the results section of this thesis, only changes that lie well outside the error bars are considered significant. This usually refers to order of magnitude changes in the number density.

\section{Redistribution:}

Material redistribution between nodules was also a major concern. The differences in the surface energies between the graphite substrate and the copper deposit result in an ideal situation for material redistribution. Because graphite has a much lower surface energy than copper, the system, while trying to minimize its total energy, minimizes the exposed surface area of copper. Therefore, spherical deposits are most stable, and producing continuous films is difficult. As the nodule grows, the surface area to volume ratio decreases and consequently the larger nodules become energetically more favored over the smaller ones. This results in the smailer nodules dissolving and eventually disappearing, while the larger ones grow; this is analogous to the Ostwald ripening effect. If the smaller nuclei disappear, the determined nucleation density will be too small. To quantify this effect, the open circuit potential of a copper deposited HOPG electrode was measured.

In this experiment, copper is deposited on the surface during a potential step to $-700 \mathrm{mV}$ versus copper for 25 seconds. The electrolyte concentration is $50 \mathrm{mM}$ 
$\mathrm{CuSO}_{4}$ in $1 \mathrm{M}$ aqueous $\mathrm{H}_{2} \mathrm{SO}_{4}$. Since there is copper on the surface, the open circuit potential of this electrode is expected to be at zero versus the copper reference electrode. However, a long cathodic transient is measured. (Figure 2-6) This transient is not caused by the concentration field relaxation. As seen in figure 2-7, when the same experiment is performed on a copper substrate, the potential difference due to the depletion of copper ions near the surface is an anodic potential.

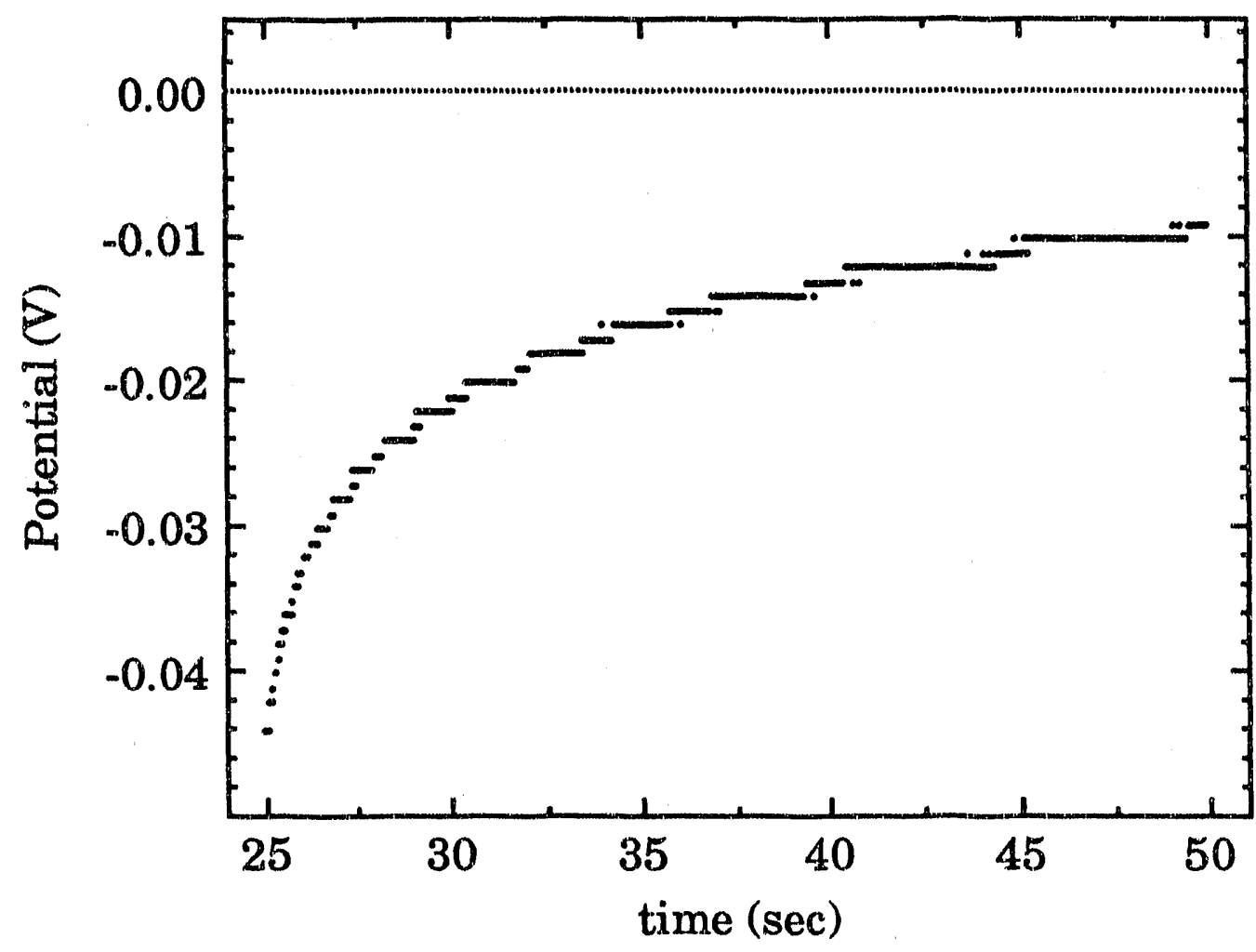

Figure 2-6: Open circuit potential on an HOPG electrode following copper deposition for $25 \mathrm{sec}$ at $-700 \mathrm{mV}$ versus copper reference electrode from $50 \mathrm{mM} \mathrm{CuSO}_{4}$ and $1 \mathrm{M}$ aqueous $\mathrm{H}_{2} \mathrm{SO}_{4}$ electrolyte. Cathodic potential transient due to redistribution of copper from small nodules to large ones. 


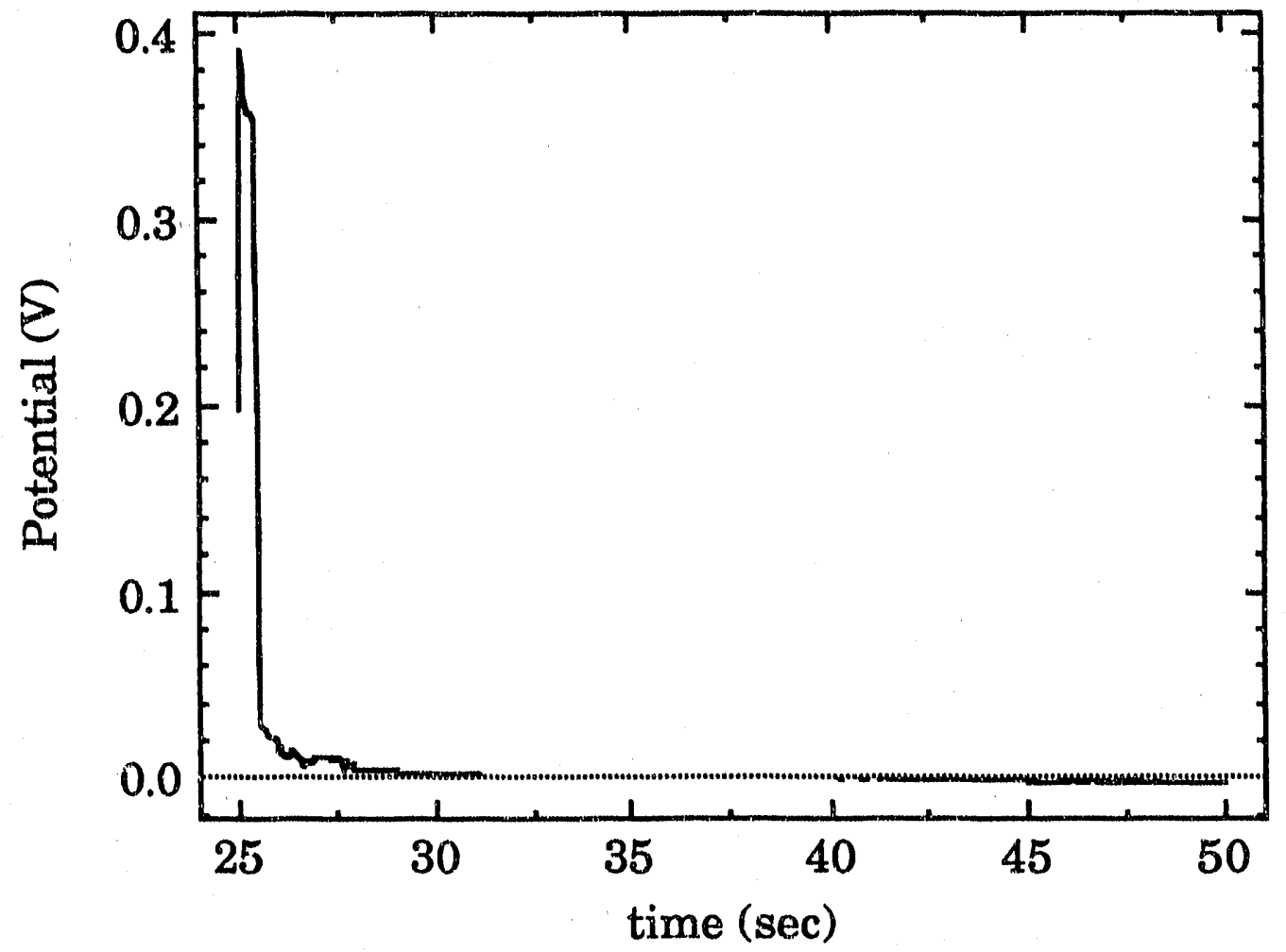

Figure 2-7: Open circuit potential on a copper electrode following copper deposition for $25 \mathrm{sec}$ at $-700 \mathrm{mV}$ versus copper reference electrode from $50 \mathrm{mM} \mathrm{CuSO}_{4}$ and $1 \mathrm{M}$ aqueous $\mathrm{H}_{2} \mathrm{SO}_{4}$ electrolyte. Anodic transient due to the relaxation of the concentration field.

This potential is similar to a corrosion potential. Because the sample is at open circuit, no net current can flow to the surface. However, there are areas on the surface where a cathodic reaction is taking place, namely, the copper depositing on the large nodules. There are other areas, where an anodic reaction is taking place, namely, the dissolution of copper from the small nodules. The rate of these two reactions must equal one another for no net charge to pass.

In cases where one reaction is kinetically favored over another, the potential of the surface shifts until the two rates are equal. Where the cathodic reaction is facile and the anodic process is slow, the potential of the surface shifts to more 
anodic potentials to hinder the cathodic process and facilitate the anodic process. In the situation where the opposite is true, as in the case in figure 2-6, the surface shifts to more cathodic potentials to keep the net current zero.

\subsection{Scharifker and Mostany's Model:}

\section{Description of Model:}

The objective of using the Scharifker and Mostany ${ }^{3}$ model in this research is to determine nucleation density from the current transient to a potentiostatic step experiment. The model predicts the total number of active sites and the nucleation rate per active site from the current maximum on the transient. It uses the Fleischmann and Thirsk 4 model for nucleation. The effects of migration and convection are neglected; mass transport is assumed to be solely by diffusion. The depletion regions around the nodules axe considered to be nucleation exclusion zones and a statistical treatment is used to determine the depletion zone overlap. After the diffusion zone overlap occurs, the diffusion is simplified to planar diffusion to the projected area of the depletion zones on to the surface. The overall expression for the current density is the product of an expression for the current due to planar diffusion and the area covered by the depletion zones, expressed in eqn. [2-1].

$$
I=\left(z F c \sqrt{\frac{D}{\pi t}}\right)\left(1-\exp \left\{-N_{0} \pi k D\left[t-\frac{\left(1-e^{-A t}\right)}{A}\right]\right\}\right)
$$

where:

$$
k=\left(8 \pi c M_{w} / \rho\right)^{\frac{1}{2}}
$$

and: $\quad I=$ current density $\left(\mathrm{A} / \mathrm{cm}^{2}\right)$

$\mathrm{zF}=$ Charge per mole of depositing species $(\mathrm{C} / \mathrm{mol})$

$\mathrm{c}=$ bulk concentration $\left(\mathrm{mol} / \mathrm{cm}^{3}\right)$

$\mathrm{D}=$ diffusion coefficient $\left(\mathrm{cm}^{2} / \mathrm{s}\right)$ 


$$
\begin{aligned}
& t=\text { time }(\mathrm{s}) \\
& N_{0}=\text { number density of active sites }\left(\mathrm{cm}^{-2}\right) \\
& A=\text { nucleation rate per active site }\left(\mathrm{s}^{-1}\right) \\
& M_{W}=\text { molecular weight }(\mathrm{g} / \mathrm{mol}) \\
& \rho=\text { density of the deposit }\left(\mathrm{g} / \mathrm{cm}^{3}\right)
\end{aligned}
$$

Taking the derivative of equation [2-1] and setting it equal to zero to determine the time, $t_{m}$, at the current maximum, leads to the following expression for $t_{m 1}$ :

$$
\begin{gathered}
\ln \left(1+2 b t_{m}-2 b t_{m} e^{-A t_{m}}\right)-b t_{m}+(b / A)\left(1-e^{-A t_{m}}\right)=0 \\
\text { where: } b=N_{0} \pi k D
\end{gathered}
$$

and: $\quad t_{m}=$ time at the current maximum

The following substitutions are made to simplify the expressions:

$$
\begin{aligned}
& x=b t_{m} \\
& \alpha=b / A \\
& a=z F c \sqrt{\frac{D}{\pi}}
\end{aligned}
$$

With the substitution of the above definitions and further simplification equations [2-1] and [2-3] become:

$$
\begin{gathered}
\ln \left(1-\frac{I_{m} t_{m}^{\frac{1}{2}}}{a}\right)+x-\alpha(1-\exp (-x / \alpha))=0 \\
\ln [1+2 x(1-\exp (-x / \alpha))]-x+\alpha(1-\exp (-x / \alpha))=0 \\
\text { where: } I_{m}=\text { current density maximum }
\end{gathered}
$$


By solving the above equations simultaneously for $\mathrm{x}$ and $\alpha$, the number density of active sites and their activation rate can be determined, from the experimentally determined current maximum and the time at the maximum. The two equations can be solved numerically using the Newton-Raphson technique. The Jacobian matrix, constructed of the derivatives of the two functions with respect to their variables, is:

$$
W(x, \alpha)=\left[\begin{array}{ll}
\frac{\partial f_{1}(x, \alpha)}{\partial x} & \frac{\partial f_{1}(x, \alpha)}{\partial \alpha} \\
\frac{\partial f_{2}(x, \alpha)}{\partial x} & \frac{\partial f_{2}(x, \alpha)}{\partial \alpha}
\end{array}\right]
$$

where: $f_{1}(x, \alpha)=0$

$$
f_{2}(x, \alpha)=0 \quad \text { are the equations }[2-8] \text { and }[2-9]
$$

Given an initial guess for the roots $x_{p}$ and $\alpha_{p}$, better approximations $x_{p+1}$ and $\alpha_{p+1}$ are calculated using eqn. [2-11]:

$$
\left[\begin{array}{l}
x_{p+1} \\
\alpha_{p+1}
\end{array}\right]=\left[\begin{array}{l}
x_{p} \\
\alpha_{p}
\end{array}\right]-W^{-1}\left(x_{p}, \alpha_{p}\right)\left[\begin{array}{l}
f_{1}\left(x_{p}, \alpha_{p}\right) \\
f_{2}\left(x_{p}, \alpha_{p}\right)
\end{array}\right]
$$

The solution to the set of equations [2-8] and [2-9] can then be found by iterating until the sum of the residuals, $f_{1}$ and $f_{2}$ is less than a specified tolerance. The number density of active sites, $N_{0}$, and the activation rate per site, $A$, are determined from the definitions of $\mathrm{x}$ and $\alpha$. The computer code written to solve this problem is included in Appendix A. This program calculates the number density of active sites and the activation rate of these sites based on the Scharifker - Mostany model with only the current maximum and the time at the maximum as input variables. The experimental parameters such as copper concentration, density and the diffusion coefficient are defined as constants in the program. 


\section{Comparison with Experimental Measurement:}

A current transient from a potential step experiment is reproduced in figure 2-8. In this experiment, the copper was deposited from $50 \mathrm{mM} \mathrm{CuSO}_{4}$ and $1 \mathrm{M}$ aqueous $\mathrm{H}_{2} \mathrm{SO}_{4}$ electrolyte onto an $\mathrm{HOPG}$ electrode. The potential is stepped to $-700 \mathrm{mV}$ versus copper for one second. The current maximum is $31.2 \mathrm{~mA} / \mathrm{cm}^{2}$ at 0.139 seconds from the start of the potential step. Entering these values into the program, $\mathrm{N}_{0}$ is calculated to $3.78 \times 10^{6} / \mathrm{cm}^{2}$ and $A$ to 252 seconds. The current transient for these values is calculated from equation [2-1], and plotted in figure 2-8 along with the experimentally measured current transient. The model fits the measured data well.

Instantaneous and progressive nucleation are shown by Scharifker to be special cases of the general expression for the current transient. For instantaneous nucleation $(\alpha \rightarrow 0)$ equation [2-1] simplifies to:

$$
I=\left(a / t^{1 / 2}\right)[1-\exp (-b t)]
$$

And progressive nucleation $(\alpha \rightarrow \infty)$ leads to:

$$
I=\left(a / t^{1 / 2}\right)\left[1-\exp \left(-A b t^{2} / 2\right)\right]
$$

These two special cases are plotted together in figure 2-9. Both plots are based on the same values for $N_{0}$ and $A$, which were determined numerically. Scharifker et al. define instantaneous and progressive nucleation as the two extreme situations of fast nucleation on a small number of active sites and slow nucleation on a large number of active sites, respectively. The model fit from figure 2.8 more closely resembles the instantaneous curve than the progressive one. This agrees well with the assurnption of instantaneous nucleation of copper on graphite. 


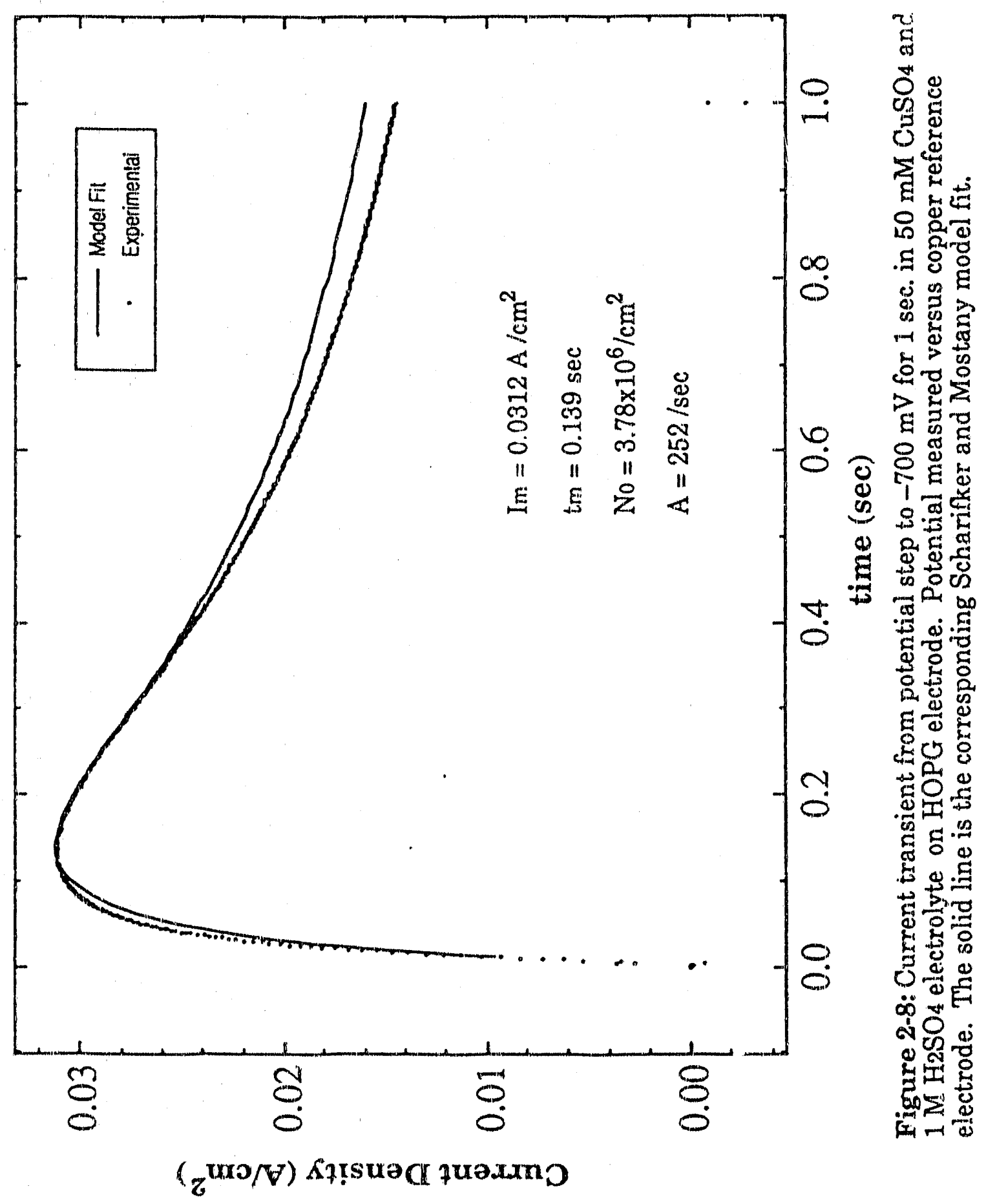


To determine the number density of nuclei, $N$, for the above fit, we return to the definition of nucleation density in the Fleischmann and Thirsk model:

$$
N=N_{0}[1-\exp (-A t)]
$$

Substituting the calculated values for $\mathrm{N}_{0}$ and $A$, the exponential term reduces to zero and the nucleation density is calculated to be $3.78 \times 10^{6} / \mathrm{cm}^{2}$ at the end of the one second long potential step. The nucleation density is equal to the number density of active sites, which also agrees well with the assumption of nearly instantaneous nucleation. The model predicts that, within $18 \mathrm{masec}, 99 \%$ of all the active sites are nucleated.

To verify nucleation density experimentally, the HOPG electrode was analyzed with the SEM. The measured nodule density, $N$, is $6 \times 10^{8} / \mathrm{cm}^{2}$. This value is much larger than that predicted by the model. Because the measured number density is larger than the calculated one, it is not possible to explain the discrepancy as experimental error in determination of nucleation density using the SEM. If this value were smaller, a possible explanation would be the loss of nuclei before analysis. We can only conclude that, though the model appears to fit the measured current transient well, it fails in predicting the correct number density of nodules. 


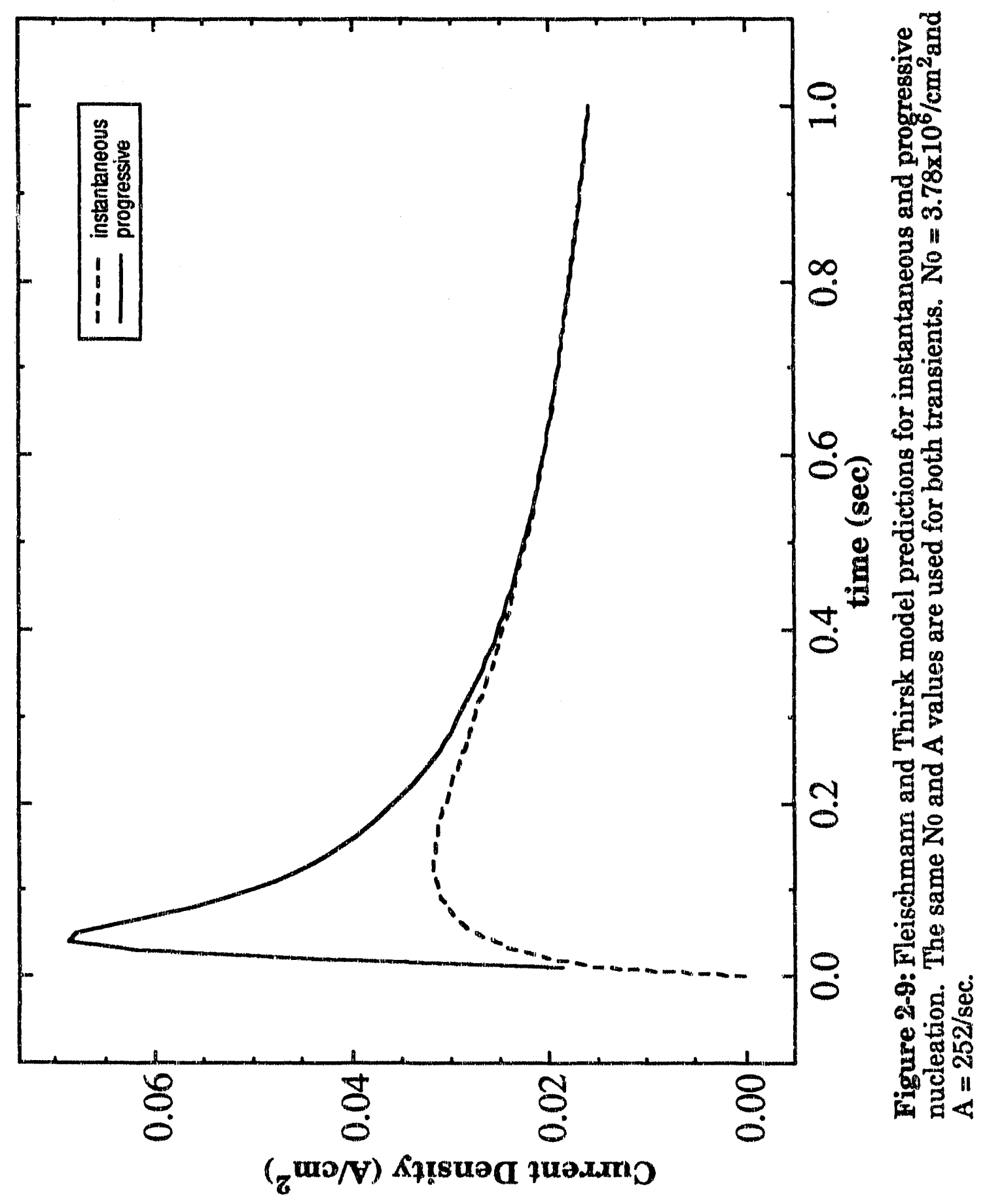




\subsection{Scanning Tunneling Microscopy:}

\section{Introduction:}

The scanning tunneling microscope (STM) was first developed by Binnig et al. at the IBM Zurich Laboratories in 1981.5 It maps the electronic topography of surfaces down to subatomic resolution. Binnig and his colleagues first reported tunneling currents in vacuum in 1981, producing images in 1982,6 and atomic scale features on a silicon surface in $1983 .{ }^{7}$ Atomic resolution images could not be reproduced by other researchers for a few years, but since then a number of developments have been made, such as imaging in air and in liquids. ${ }^{8}$ STM is now a commercial product. The instrument used in this research is a Nanoscope I developed by Digital Instruments Inc.

In this technique, a very sharp probe is brought within Angstroms of the surface that is being examined. A small tunneling current (few nanoamperes) is passed between the tip of the probe and the surface by applying a voltage difference between the two. This biasing voltage is usually in the range of $2 \mathrm{mV}$ to $2 \mathrm{~V}$. The probe is then scanned across the surface and topographical information is gathered from the relationship of the tunneling current to the separation distance. Since the current varies exponentially with the gap size, very small variations in the gap size cause large variations in the tunneling current.

There are two modes of operation: the constant current and the constant height mode. In the constant current mode, which was developed first, the probe is scanned across the surface and the current variations sensed. A feedback network changes the height of the probe to keep the current constant. Since the current is very sensitive to gap size, constant current keeps the gap constant. The probe height is then plotted versus the scan position. This technique is suitable for imaging surfaces with large scale roughness. The feedback mechanism adjusts the 
probe height constantly so that there is little likelihood of the tip crashing into a suddenly encountered protrusion.

In the constant height mode the probe is scanned across the surface at nearly constant height and constant bias voltage while the current is monitored. The rapid variations in the tunneling current, which indicate the surface topography, are then plotted versus scan position. This technique is much better suited for imaging nearly atomically flat surfaces, because the probe beight is not adjusted. It is also used for scanning much more quickly, because the speed of the feedback loop is no longer a limitation. Fast imaging is important for reducing data collection time for possible study of real time processes.

\section{Piezoelectric Scanning Mechanism:}

A piezcelectric crystal when deformed by an external stress will acquire a net dipole moment. This moment produces surface polarization charges, which can be detected by measuring the potential developed across the opposite sides of the crystal. For moderate stresses the polarization and hence the potential is proportional to the applied stress. The converse is also true. If the crystal is placed in an electric field, it distorts. It is this property that is employed in the piezoelectric drive.

The original piezoelectric drive, as applied by Binnig and his group, used three slabs made of a piezoelectric material, to control the motion in all three directions. The drive, as it is used in present commercial STM's, is a tubular piezoelectric material, which was developed by Binnig and Smith in $1986.9^{9}$ (Figure 2-10) The electrodes on the tube are sectioned off into $X, Y$ and $Z$ controlling areas. The tube is distorted by applying a voltage across the wall of the tube. The difference in the distortions between $\mathrm{X}$ and $\overline{\mathrm{X}}$ moves the tube and hence the probe 
that is attached to it. Moving in the $\mathrm{Z}$ direction is achieved by varying the $\mathrm{Z}$ voltage, causing the tube to contract.

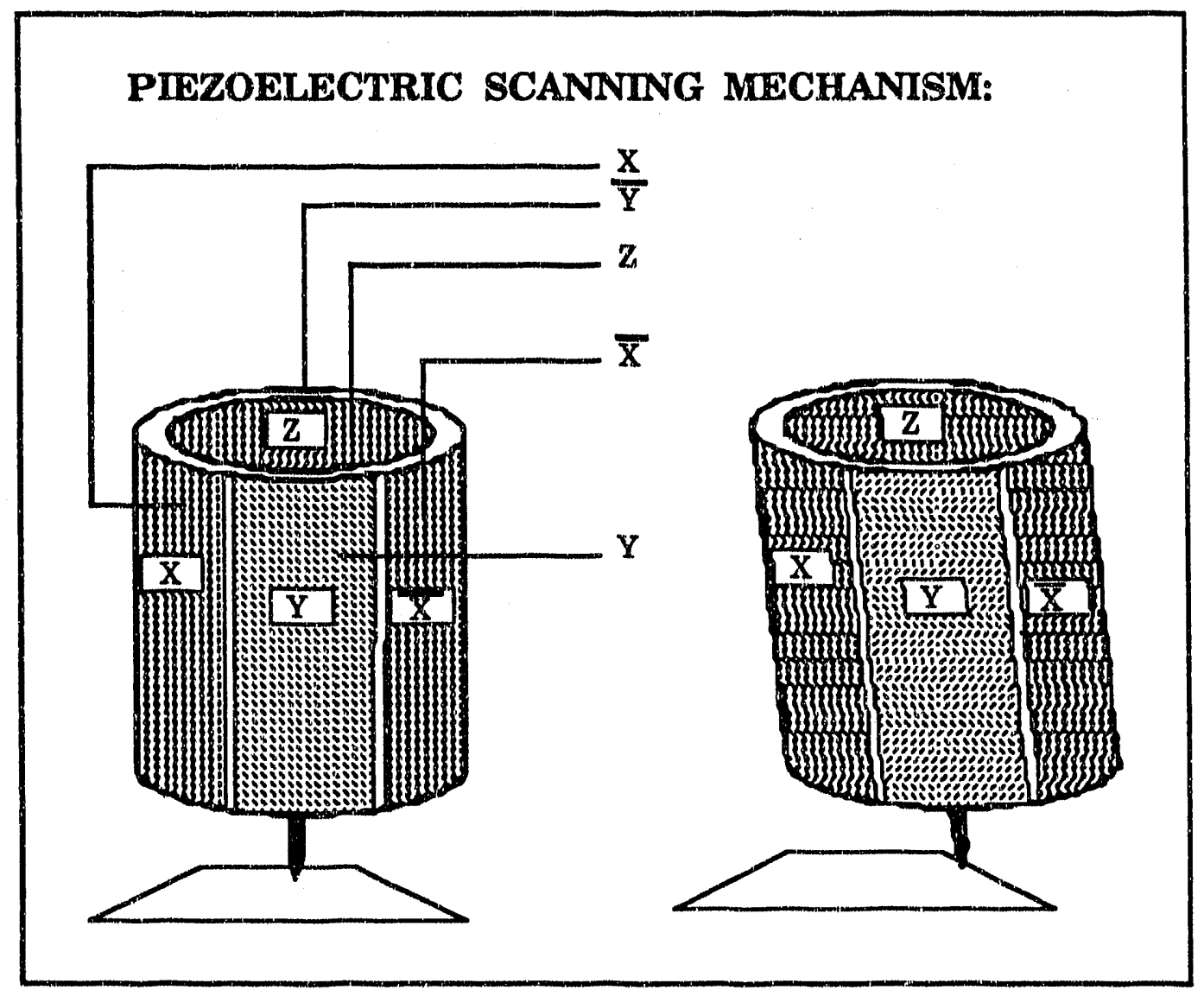

Figure 2-10: The piezoelectric tube, which is used to control the small movements of the tunneling probe in STM.

\section{Data Collection and Presentation:}

A micro computer was used for collecting and storing the (X, Y, Z) data. All the $\mathrm{x}-\mathrm{y}$ control, data collection, data manipulation, and data display functions were performed using software developed in this laboratory by a former researcher, Michael Armstrong, and is well documented in his Ph.D. Thesis. ${ }^{10}$

There are different ways of presenting the $(X, Y, Z)$ data collected. 
a. Line drawing: Line drawings are historically the first types of images produced. Initially, they were just current versus $\mathrm{X}$ plots displaced in the $\mathrm{Y}$ direction. Some of the plots are re-drawn to simulate a viewing angle a few degrees above the surface.

Contour map: Contour maps are similar to topographical maps. Connecting lines are drawn through equal height or current points.

Gray Scale Mapping: A gray level is assigned to each $\mathrm{Z}$ value and is plotted in the $\mathrm{X}$ and $\mathrm{Y}$ plane. Usually light color indicates elevations and dark color recesses.

Slope coding: Slope coding is a method where a gray level is assigned according to the value of the derivative. Usually positive slopes are assigned lighter shades and negative slopes are assigned darker shades of gray. These plots give the impression of a light being shone on the surface at a grazing angle.

Simulated diffuse reflectance: This algorithm simulates illumination from directly above the surface by assigning a gray level to the square of the cosine of the angle at the $(\mathrm{X}, \mathrm{Y})$ position to the average surface normal.

Color: Color, which is assigned to a range of $\mathrm{Z}$ values, can be added to any of the above techniques for visual appeal.

\section{Experimental Techniques:}

For ex-situ experimentation, the samples were first prepared in an electrochemical cell, then rinsed, dried, and brought to the scanning tunneling microscope for imaging in ambient air. The tunneling probe tips were prepared in the laboratory from either platinum or tungsten wire, shortly before the analysis was performed. Two methods were employed for creating sharp tips. In one technique, the probes were mechanically cut while being stretched; and in the other, they were electrochemically etched to a point. 11 


\section{Complications:}

Since the very high resolution of the STM depends directly on the ability to move the probe at very small increments extremely precisely, there are some inherent difficulties that must be overcome. External effects, even if small, can cause large scale disturbances in the system. The microscope must be well isolated from any vibrations that may exist in the building. The instrument employed in this study sits on a large, aluminum table supported by three air suspension legs.

Small temperature changes can result in thermal drift, which results in the surface moving with respect to the tip. Since the data presentation programs all assume the surface remains still, the images collected become skewed. Piezoelectric creep can also be a problem. It results from the piezo relaxing with time, which causes the tip to move. The effect of the thermal and piezo drift can be minimized by scanning quickly In constant height mode, an entire scan can be collected in less than 32 sec.

Certain difficulties with the reproducibility of the data exist. Because the STM is very sensitive and small perturbations cause large distortions in the data, reproducing the same images can be challenging. Without reproducibility, the validity of the results is questionable.

Tip quality is the single most crucial requirement for good STM results. Ideally, the probe would have a single sharp point, which tapers off to a single atom. This, however, is not likely to be the case. There usually are many points at the end of a probe, which is more rounded. In many cases, multiple tunneling can occur. In multiple tunneling, the current flows through different parts of the probe as the probe scans over a feature. (Figure 2-11) This usually results in a hysteresis between the forward and the reverse scan. 
One must always keep in mind that STM images are a convolution of the surface and tip shape. Under extreme circumstances, such as a surface feature with a sharper point than the probe, the surface feature can image the tip. (Figure 2-11) If there are many sharp features on the surface, the resulting STM image will show the same tip image repeated many times. An example of this effect is shown in figure 2-12. The similar shape and orientation of the surface features indicate that the shape of the tip is being projected onto the surface image. Ideally a surface should be examined many times using different probes to conclude with confidence that the images generated actually represent the shape of the surface alone, and not a superposition of the tip shape on the surface.

\section{Image interpretation:}

The scanning tunneling microscope is a very powerful tool for examining surface features at nanometer scales, and yet it is relatively easy to operate. Therefore it is easy to collect a great deal of data, which can become difficult to interpret. One must undertake the interpretation of results with extreme care.

Because such a small area of the surface is analyzed in any given image, one obvious difficulty that arises is finding the specific surface feature being searched, and once it is found deciding whether the feature is representative of the whole surface. This problem can be overcome by examining the surface at many locations and comparing the images.

Another difficulty in interpretation of results from the STM arises because the images generated are not only dependent on surface topography. One must keep in mind that the STM traces contours of constant electron density at a given energy level. If all the atoms on the surface are identical to one another, the image generated is representative of surface shape. This, however, is generally not the case. The path traveled by the probe is dependent on the electronic properties of the 

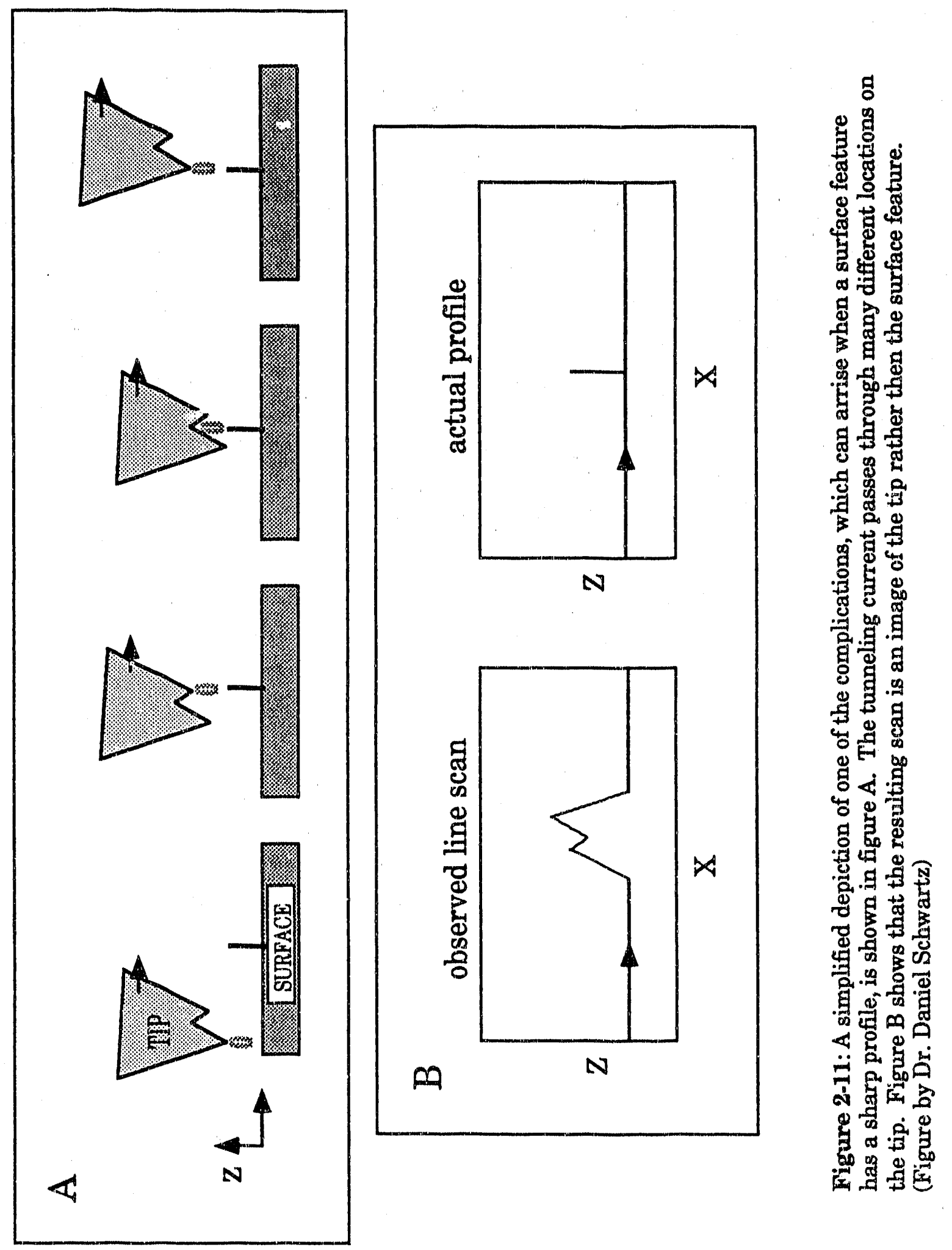
atoms on the surface and the bias voltage applied, as well as the surface topography. A discussion on interpreting STM images is given in a review article by Hansma et $a l .12$

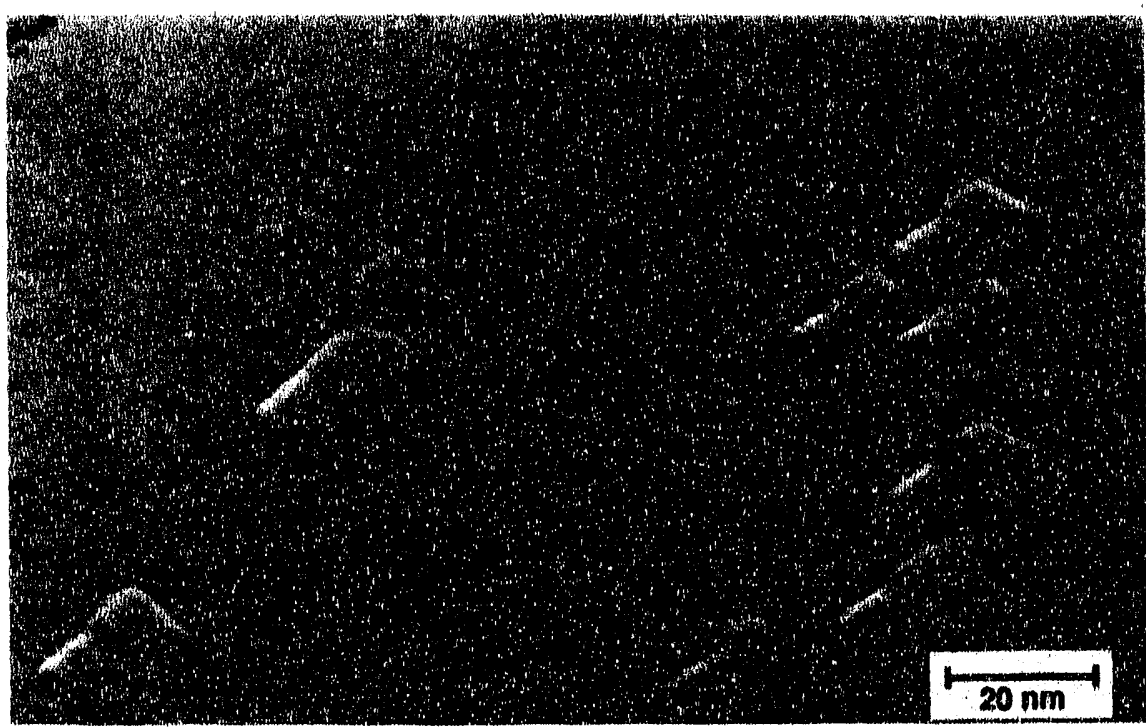

Figure 2-12:.STM image showing the superposition of the probe tip image onto the image of the surface. The surface is a polyaniline coated platinum electrode. The image is derivative coded.

Because of its large areas of nearly perfect crystalline structure and the highly localized electron fields around its atoms, HOPG has been widely studied with the STM. Atomic resolution was achieved on graphite in 1986 in vacuum. ${ }^{13}$ Since then it has been studied in air $^{14}$ and water. ${ }^{15}$ The literature is laden with in-situ STM studies of the early stages of electrodeposition. ${ }^{16,17,18}$ Caution must be taken in reading these articles. A paper recently published by Chang and Bard 19 challenges much of these earlier findings. Many of the features reported in the literature as the electrodeposit were found to be features on the graphite itself. 


\section{Experimental Results: ' Bare HOPG:}

In this study, HOPG was imaged at atomic resolution to test the limit of the instrument and its calibration. Atomic resolution was achieved in air and under a drop of water. These gray scale images are presented in figures 2-13 and 2-14. For the image under a drop of water, the coated probe was used to reduce the background current.

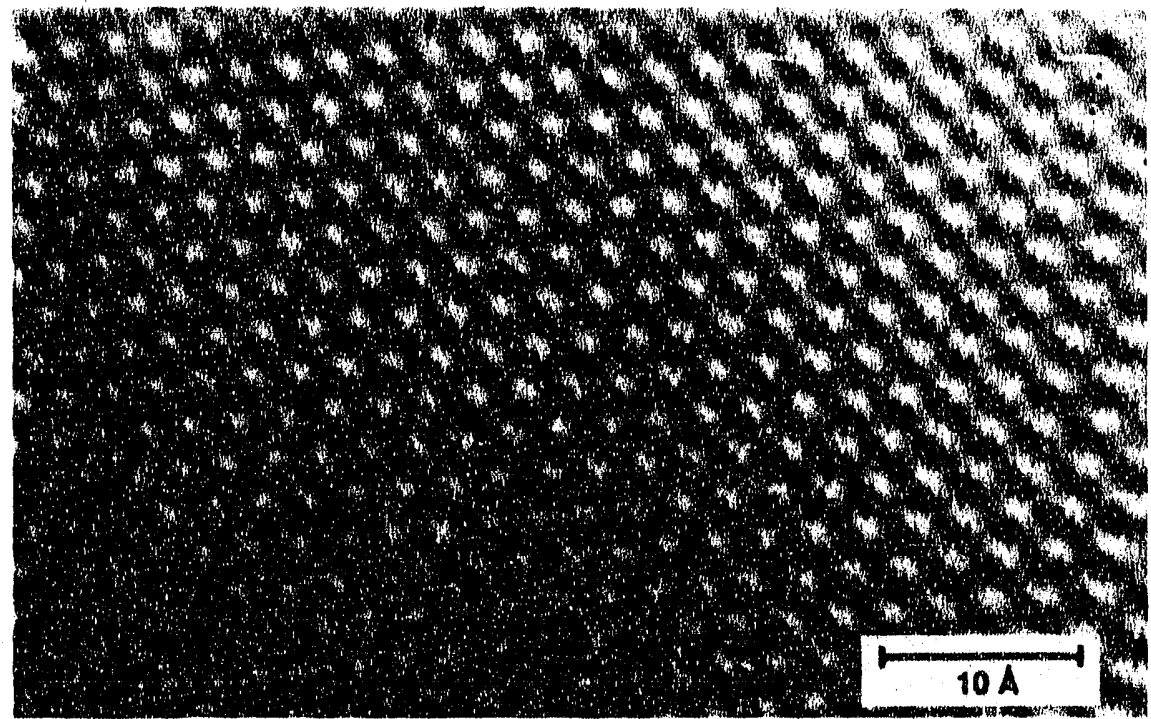

Figure 2-13: STM image of HOPG at atomic resolution. The scan was performed in air. The image is height coded, with light areas being protrusions and the dark areas recesses.

The coating prevents electrochemical reactions from taking place on the sides of the probe, which can lead to a current that is larger than the tunneling current, and make detection of the tunneling current impossible. Many different coatings were tried on the probes, including a Kynar dispersion (by Pennwalt), a Kynar 
solution (by Whitford), and clear nail polish. They all work, yet preparing consistently well coated probes is difficult.

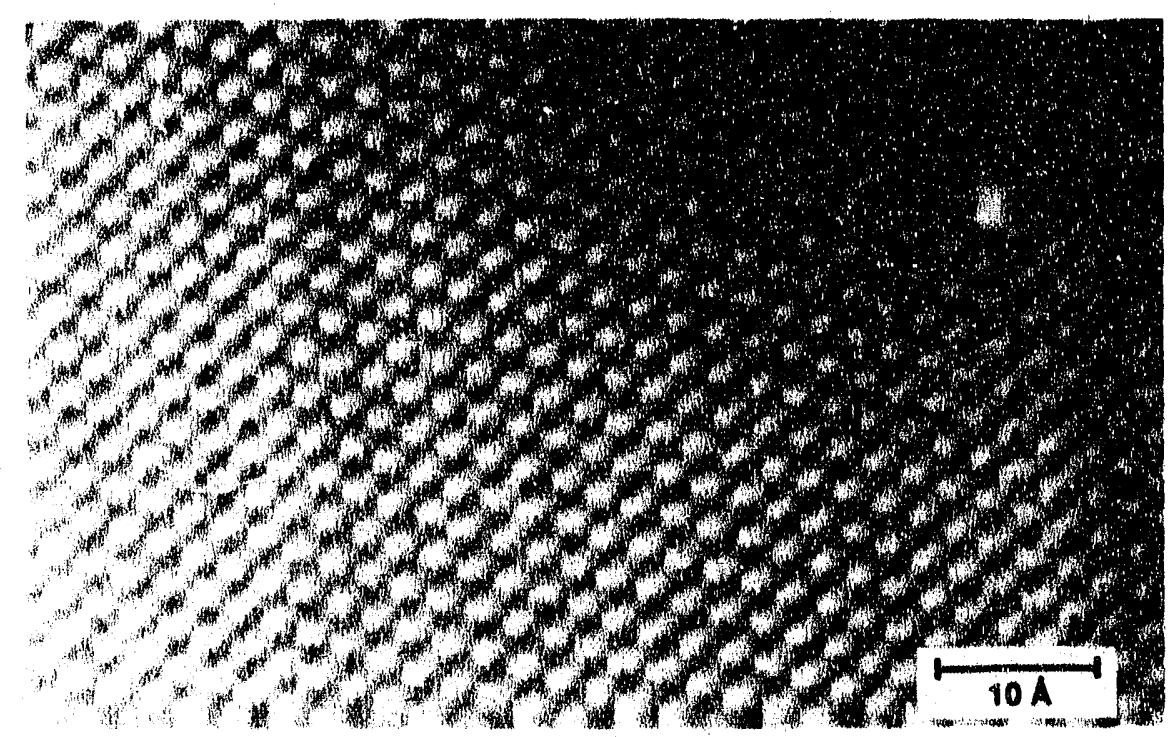

Figure 2-14: STM image of HOPG at atomic resolution. The scan was collected in water, with a Kynar coated tip. The image is height coded, with light areas being protrusions and the dark areas recesses.

In the atomic scale images in figures $2-13$ and 2-14 not every carbon atom in the hexagonal grid is seen, because alternating atoms are aligned over carbon atoms in the second layer. The atoms that are lined up above an atom in the second layer are bound to them and are not visible. The other atoms are visible because they have a dangling bond that causes a localized increase in the electron density. The dark spot in the center of each graphitic ring is caused by the low electron density there due to the lack of an atom in the surface layer. (Figures 1-5 and 2-15) 


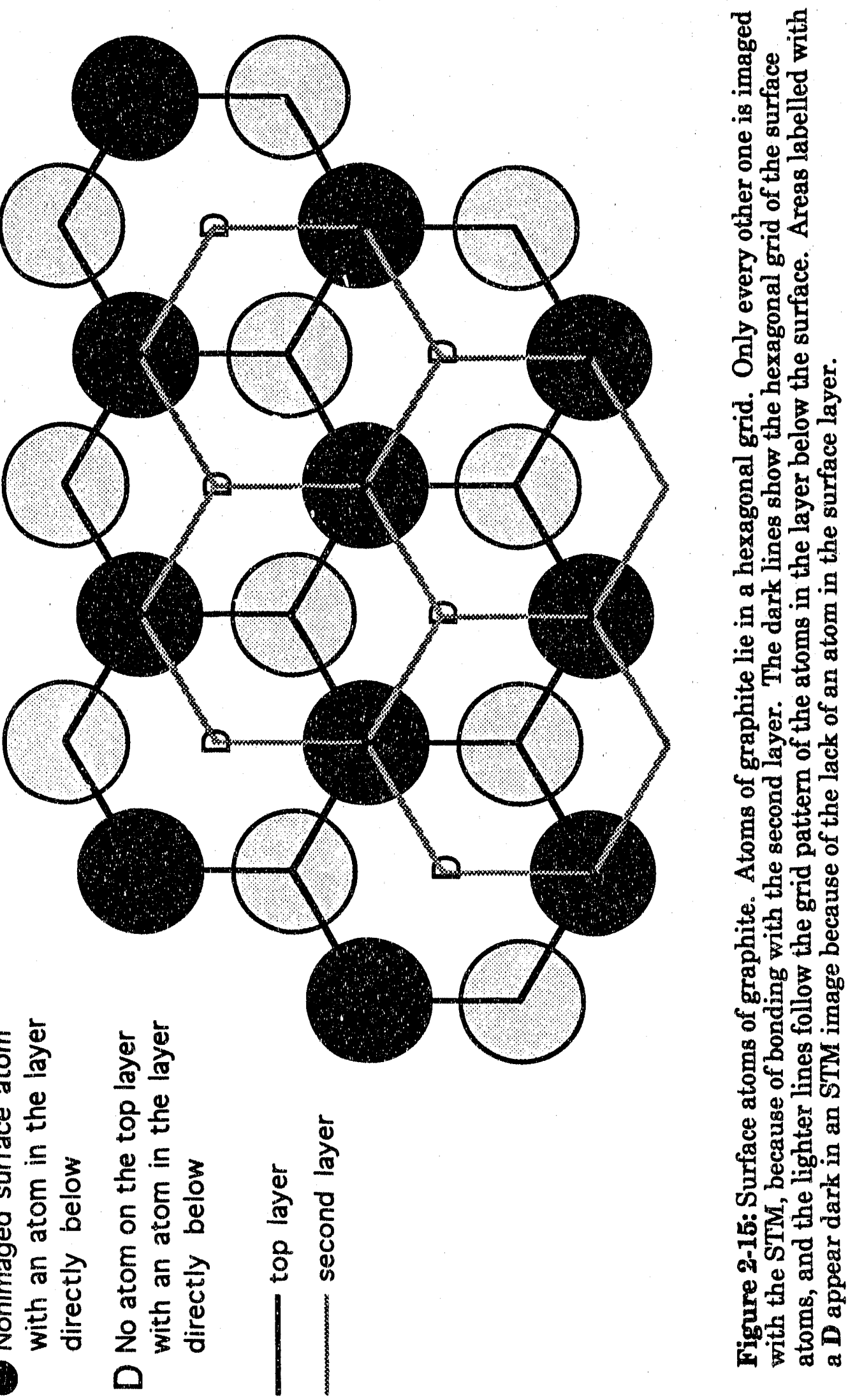


Graphite was also studied at lower magnifications, to better characterize the samples. Images similar to those reported by Bard et al. ${ }^{19}$ were obtained.

\section{HOPG with copper deposit:}

When samples with copper deposits where studied, the images were either very noisy or not very different from bare graphite. After the samples were analyzed with the SEM and the best magnifications for viewing the nodules determined, they were examined again with the STM at magnifications where the copper nodules should have been easily detected. The nodules still could not be imaged. A possible explanation for this is the poor adhesion of copper to the graphite substrate.

Because the nodules are not firmly attached to the surface, interaction with the STM probe can cause them to move. This accounts for both the moisy images and the images resembling bare graphite.

When high coverage copper deposits were produced, it was possible to image them with the STM. Figure 2-16 compares two images of the same surface: an SEM and an STM image. No image enhancement was performed on the STM image. It is a simple gray scale map of the height of the tunneling probe. The copper nodules are noisy yet easy to identify. Though the magnifications are different, clearly the nodule size (about $100 \mathrm{~nm}$ diameter) and the packing density are similar in the two images.

At high coverages, the nodules are densely packed and limited in their mobility. The fact that the nodules are imaged when they are densely packed agrees well with the explanation that they move out of the way because of probe interaction in less dense deposits. On HOPG substrate, the STM can only image surfaces with high copper coverage. This restriction limits the value of the STM as a tool for determining nucleation densities. 

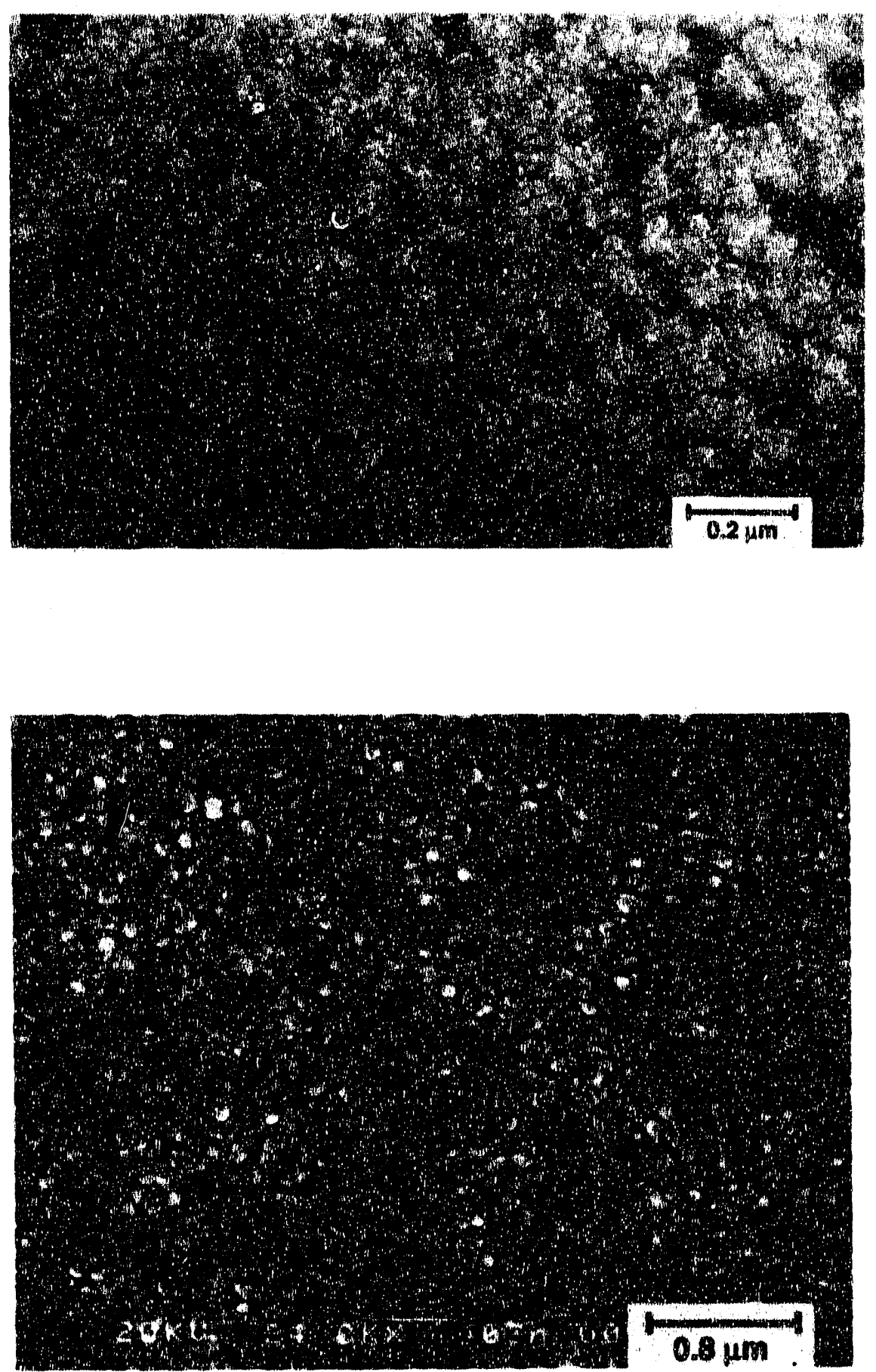

Figure 2-16: Comparison of the same sample imaged with an STM and an SEM. Sample was prepared by depositing copper onto HOPG from $50 \mathrm{mM} \mathrm{CuSO}_{4}$ and $1 \mathrm{M}$ aqueous $\mathrm{H}_{2} \mathrm{SO}_{4}$ electrolyte. Five repeated pulses (described in section 2.3) were used to achieve high nucleation densities. $\left(1 \times 10^{10}\right.$ nodules $\left./ \mathrm{cm}^{2}\right)$ The total charge density is $200 \mathrm{mC} / \mathrm{cm}^{2}$ with an average nodule diameter of $100 \mathrm{~nm}$. 


\subsection{Raman Spectroscopy:}

\section{Introduction to Raman Spectroscopy:}

In 1928, Chandrasekhara Raman discovered that scattered light, which contains an intense Rayleigh component at the same wavelength as the incident light, also produces faint signals at both longer and shorter wavelengths.

$$
h v^{\prime}=h v \pm\left(\varepsilon_{m}-\varepsilon_{n}\right)
$$

where: $\mathrm{hv}=$ incident radiation

$\mathrm{h} v^{\prime}=$ scattered radiation

$\varepsilon_{\mathrm{m}}, \varepsilon_{\mathrm{n}}$ are two energy levels in scattering substance

Because Raman scattering is not an absorption effect, as is fluorescence, it is not restricted to an absorption frequency. The incident light can be at any frequency, with the Raman shift, $\Delta v\left(=v-v^{\prime}\right)$, being independent of this frequency.

The origin of the effect is the variation of the dipole moment of the scattering molecule. The electric field of the incident light wave induces oscillations of a dipole

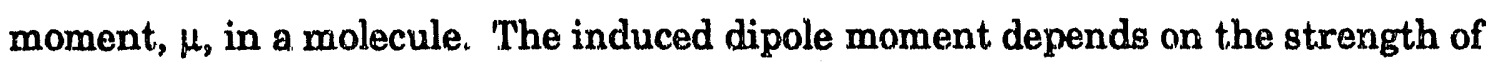
the electric field.

$$
\mu=\alpha E
$$

$$
\text { where: } \begin{aligned}
\alpha=\text { polarizability } \\
\\
\mathrm{E}=\text { amplitude of the electric field }
\end{aligned}
$$

If the polarizability, $\alpha$, varies with the vibration or the rotation of the molecule, then $\mu$ oscillates with frequencies a fixed amount above and below the incident frequency. The oscillation causes the radiation to be emitted at these frequencies. Therefore, for a material to be Raman active, the polarizability must change during rotation or vibration. Because the Raman shift is material specific, it can be used as an 
analytical tool. Differences in Raman shifts can be detected even between materials of identical elemental composition but varying crystalline structure. An example of this is plotted in figure 2-17.

The data for diamond, HOPG, and graphitized particles, presented in this figure, were measured in our laboratories by Daniel Schwartz. The base line for each Raman scan is shifted along the intensity axis to ease comparison.

All of these samples, though made of carbon, show different characteristic peaks, corresponding to the value of their Raman shifts. The diamond peak is at about $1340 \mathrm{~cm}^{-1}$. The HOPG peak is at $1580 \mathrm{~cm}^{-1}$. The graphitized particles exhibit two peaks. One of these is at the same frequency as the HOPG peak. This one is associated with crystalline graphite. The other peak is at about $1360 \mathrm{~cm}^{-1}$, and results from the Raman shift from the disordered carbon.

Previous researchers have employed Raman spectroscopy to analyze various forms of carbon. Their results are summarized in Kinoshita's book on carbon properties. ${ }^{20}$ The reported Raman shift values agree well with the data presented in figure 2-17.

\section{Experimental Procedure:}

The experimental setup is illustrated schematically in figure 2-18. The light source was an argon ion laser at $488 \mathrm{~nm}$ wavelength. The polarized laser beam is focused through a window onto the graphite substrate in the electrochemical cell at normal incidence. The scattered light from the surface is collected at an angle of $45^{\circ}$ and focused onto the entrance slit of the OMA spectrometer. The geometry of our system ensured that all of the scattered light collected was 8-polarized. The electrochemical cell (figure 2-19) houses the vertically placed HOPG working electrode along with a copper reference electrode and a copper counter electrode. The electrolyte for all of the Raman experiments was $5 \mathrm{mM} \mathrm{CuSO}_{4}$ in $1 \mathrm{M}$ aqueous 


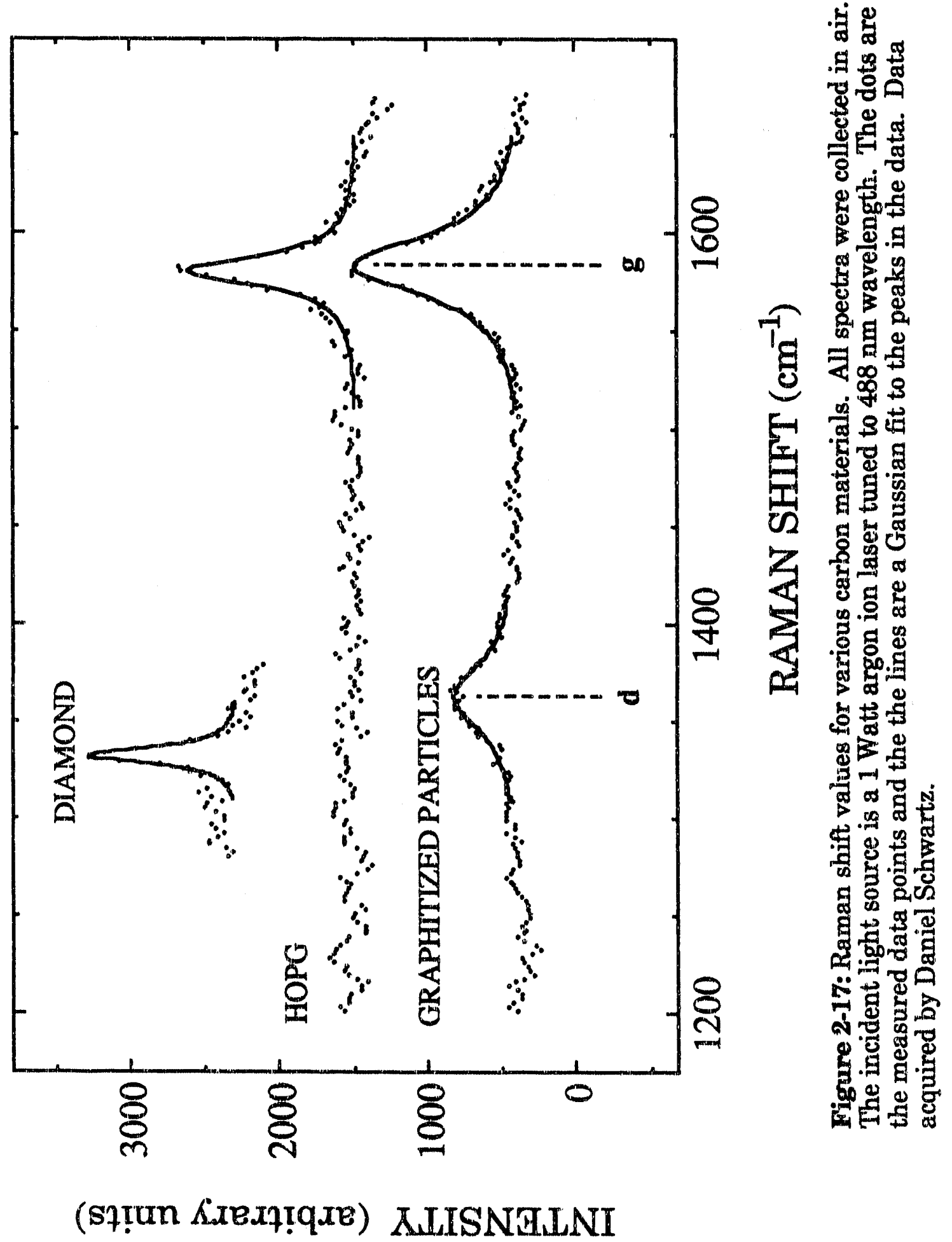




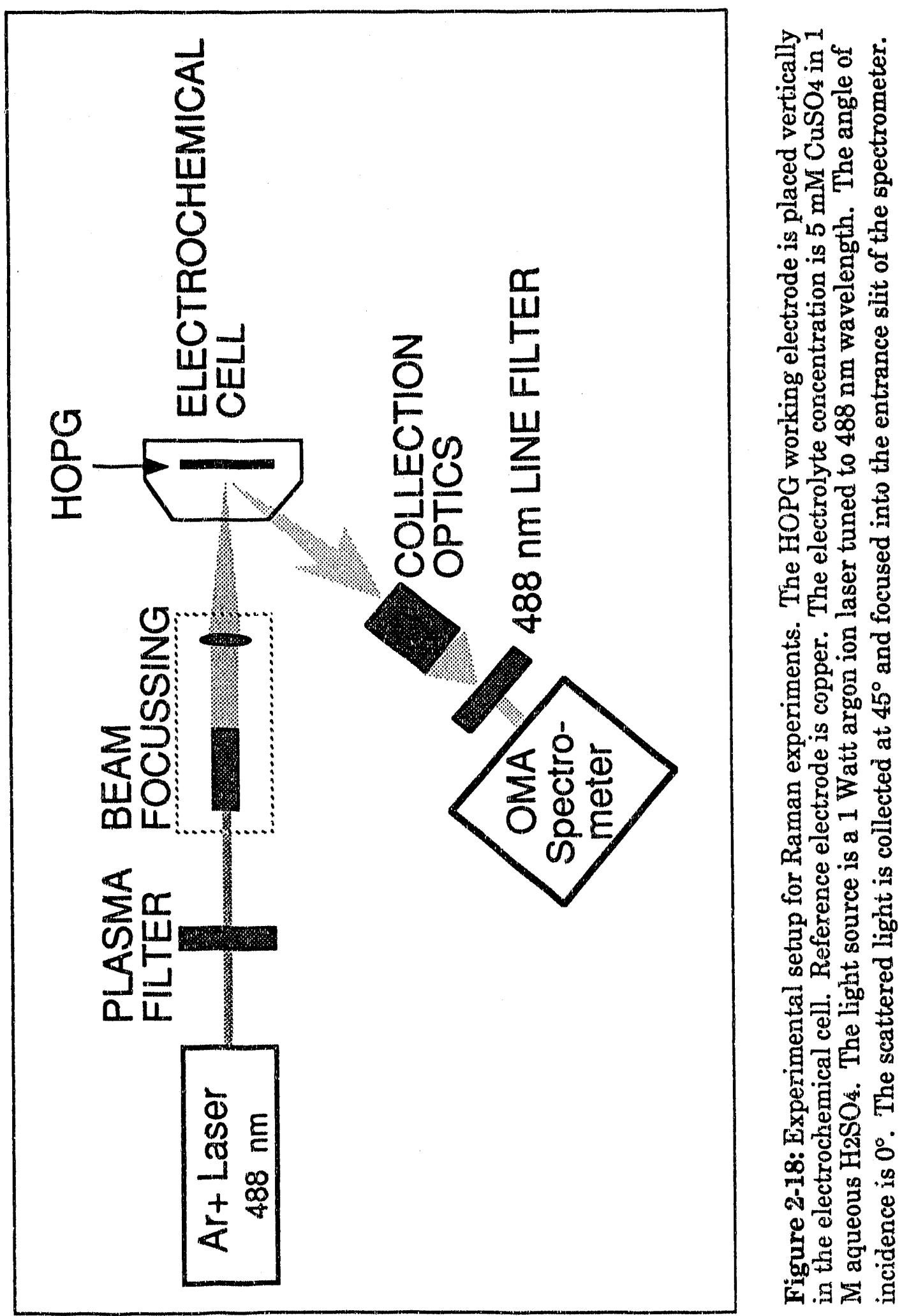


$\mathrm{H}_{2} \mathrm{SO}_{4}$. All of the Raman experiments reported in this thesis were performed in collaboration with Daniel Schwartz.

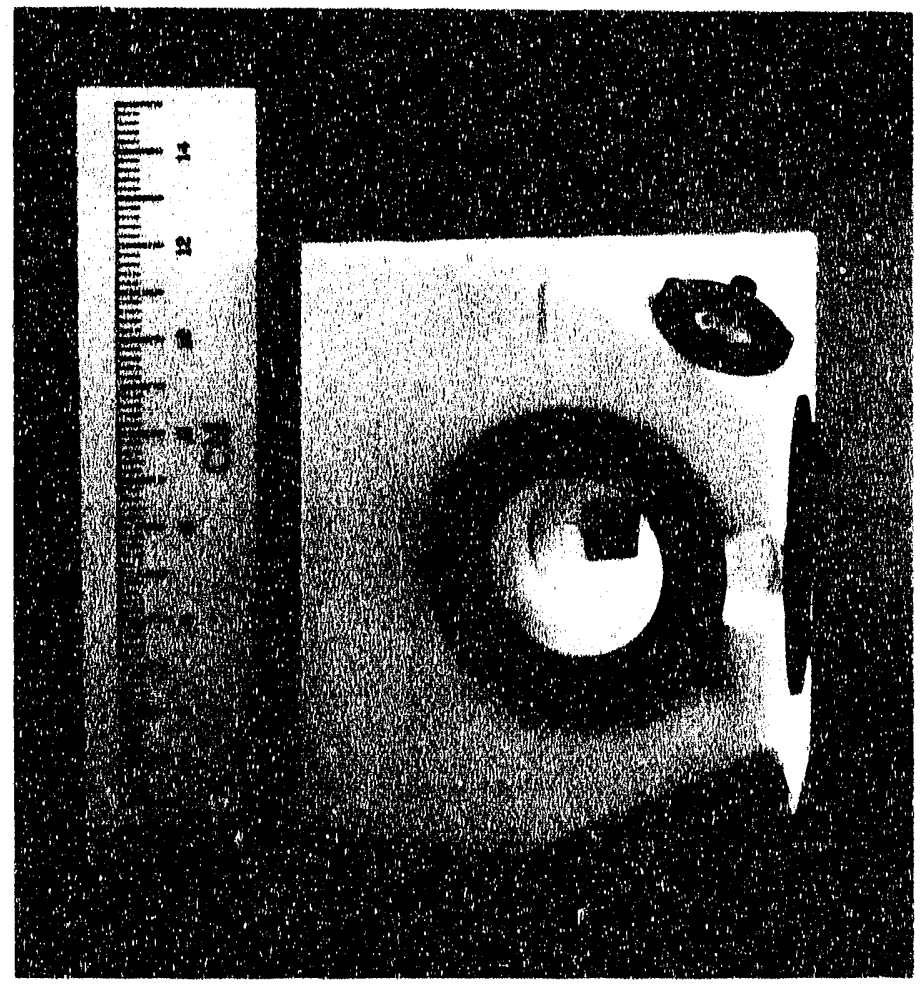

Figure 2-19: Photograph of electrochemical cell used in the Raman experiments.

The Raman spectra are collected while electrochemically depositing copper onto the graphite. It is important to stress that the graphite substrate and not the copper deposit is Raman active. On a partially copper covered surface the scattered light intensity is measured at the graphite Raman shift. Because the copper coverage limits the amount of light that reaches the graphite surface and also the amount of scattered light that reaches the collection optics, the intensity of the scattered light diminishes with increased copper coverage. Other factors such as the incident and collection angles and focusing also affect the intensity of the scattered light. When all the other factors are kept constant, however, the intensity of the 
scattered light from a copper covered graphite electrode relative to the intensity from a bare graphite electrode, is representative of the copper coverage.

The Raman spectrum, unfortunately, could not be collected while copper deposition was in progress because of the effect of the laser beam on the electrodeposition process. In order to obtain spectra with definitive features, the scattered light had to be collected for a minimum of one to two minutes. With the laser beam focused on the surface for an extended amount of time, the copper deposition was found to increase under the laser beam. Laser enhanced deposition on graphite has been studied by other researchers. 21 Some of the possible explanations are:

a. The formation of increased defect sites, due to thermal expansion on graphite surface 22

b. Improved deposition kinetics, because of the localized temperature rise

c. Mass transport enhancement by thermally driven convection

The area of increased deposition was easy to detect under the optical microscope as well as in the SEM.

Because of laser enhanced deposition, the Raman sampled area of the electrode is not representative of the entire sample. Measures taken to minimize this effect, such as focusing the laser beam into a line rather than a point, were not successful. The problem was eliminated by turning the laser off during deposition. The experimental sequence is represented in figure 2-20. 


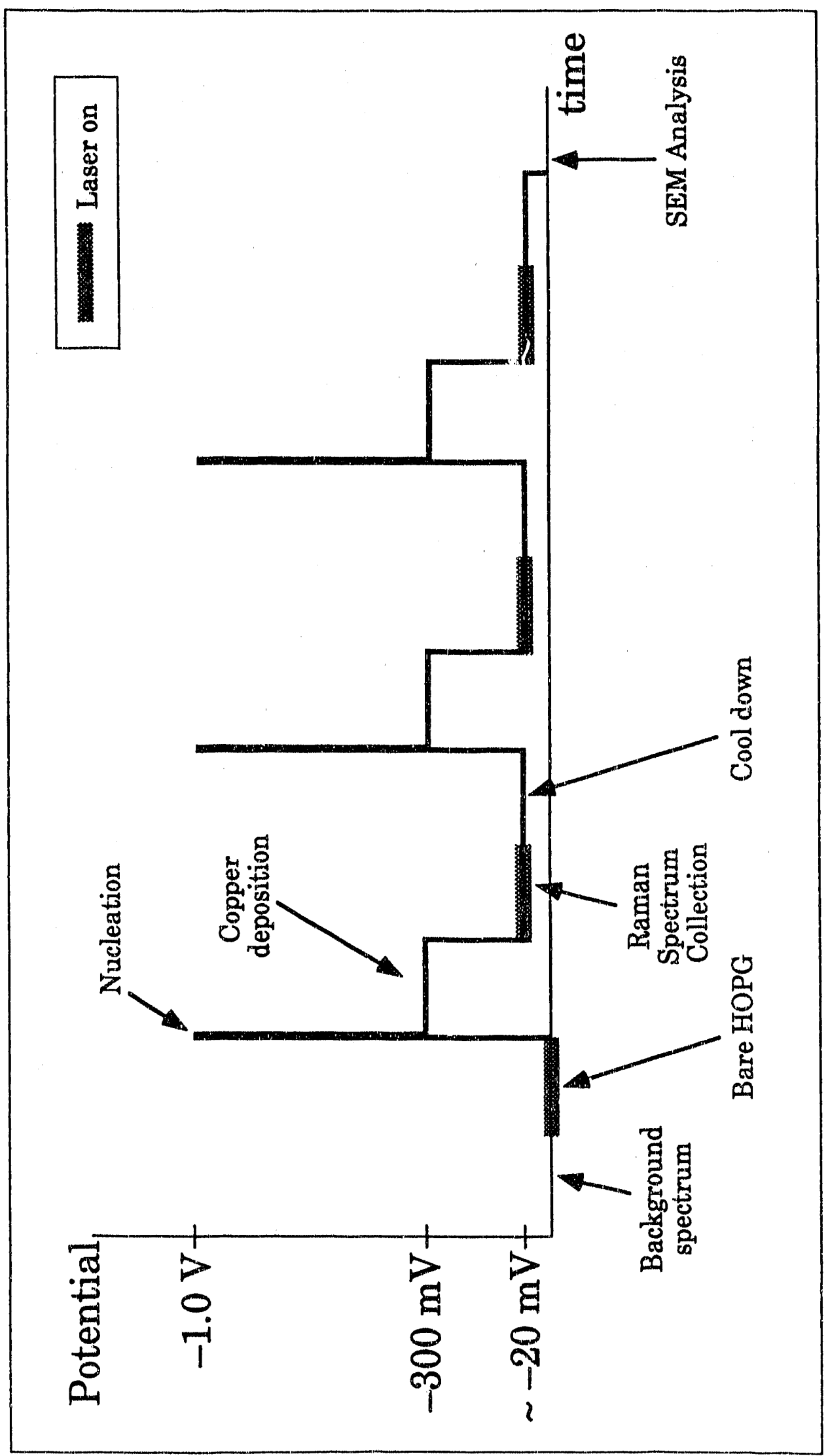

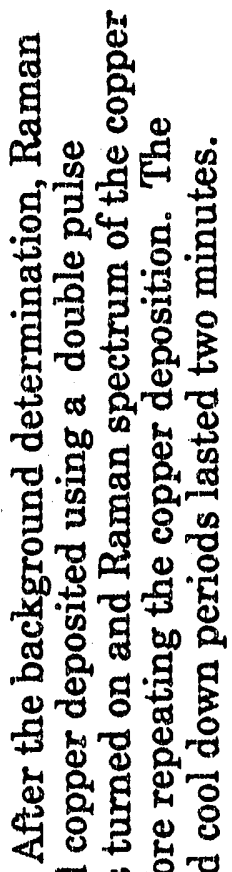

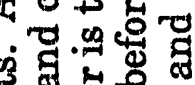
형 矛 동. 명용 년휴융

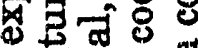
ร. 要要要

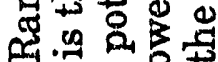

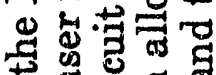
与ั 원월

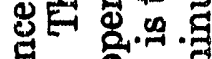
过

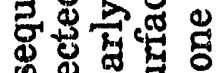

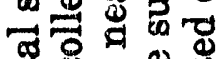
象象星 . . 造造. 4 (4) 50

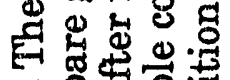
定安家 बे งे 일. 웡

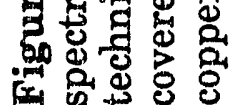


After the HOPG electrode and the electrochemical cell were prepared for the experiment and placed in position, the laser was focused on the surface. Following alignment, the laser was turned off and the background light collected for two minutes. The background was later subtracted from all the spectra, to minimize noise in the spectra caused by external light. The laser was then turned back on to collect the spectrum on bare graphite for two minutes. After the spectrum was collected, the laser was turned off and copper deposited on the graphite by means of a double pulse technique. (Described in chapter three) When the copper deposition was complete, the sample was held at a low cathodic potential. At this potential copper deposition is arrested, and the material redistribution minimized. The laser was turned on and the Raman spectrum of the copper coated graphite cullected for two minutes. At the end of this period, the laser was turned off and the surface allowed to cool before depositing more copper. After a desired number of copper deposition cycles were completed, the sample was rinsed, dried and imaged in the SEM.

The background subtracted Raman spectra for an experiment with five deposition intervals are plotted in figure 2-21. To simplify comparison the spectra are offset by about 150 counts from one another. The first spectrum is taken on bare graphite, before copper deposition. Each of the successive spectra were collected after intervals of copper deposition on the same sample. The Gaussian distribution of the intensity around the peak value allows us to make the assumption that the area under the peak scales with peak height. Comparing the peak heights to the height of the bare graphite surface, we determine the relative Raman intensity. The charge densities, the equivalent thicknesses of a uniform copper deposit and the relative Raman intensities for these spectra are summarized in table 2-1. 


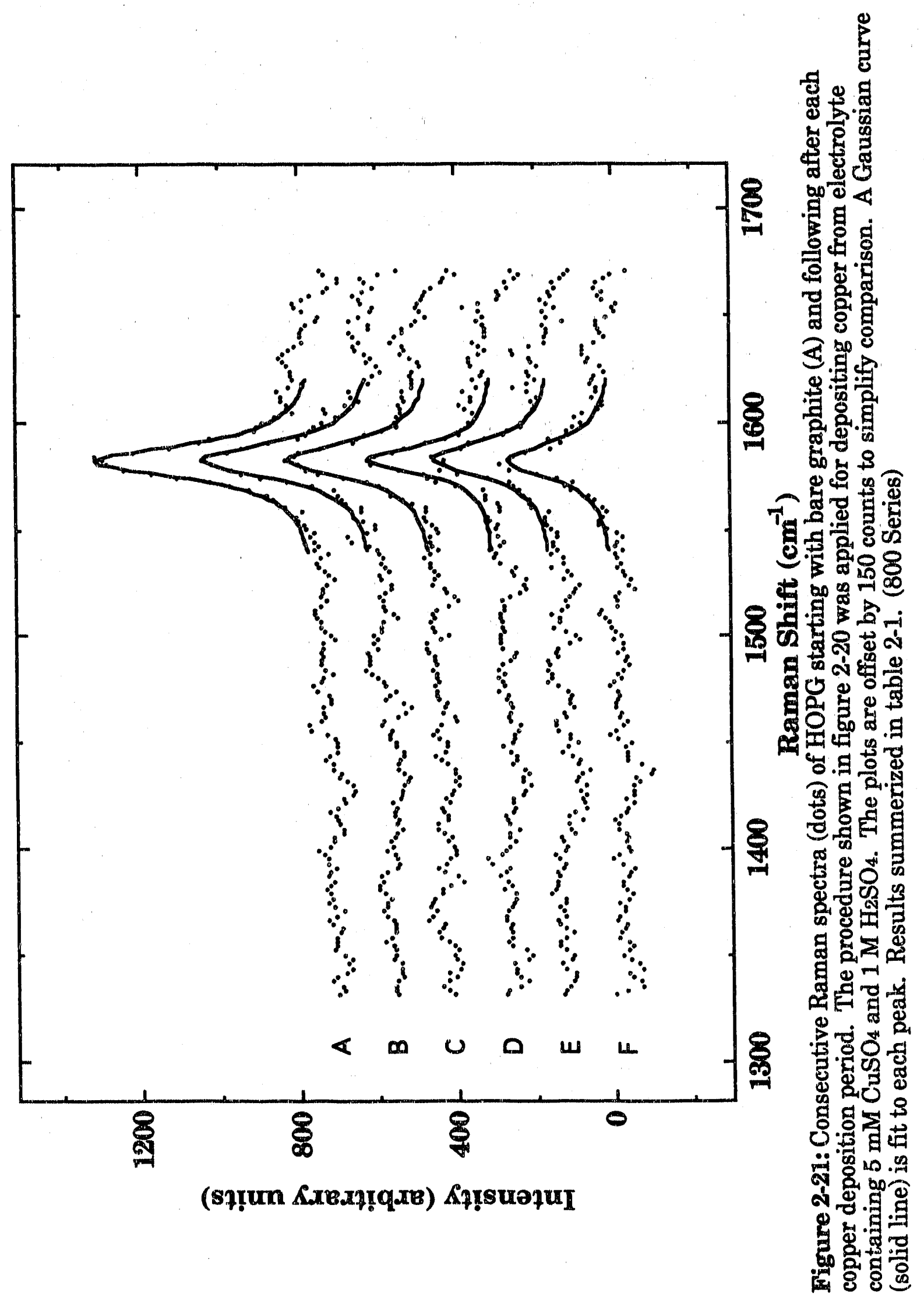


The results from four experimental sets is plotted in figure 2-22. There is a clear trend of decreased relative Raman signal with copper coverage. To obtain information on nucleation density from the Raman signal, an optical model was developed.

\begin{tabular}{|c|c|c|c|}
\hline $\begin{array}{c}\text { Spectrum } \\
\text { Number }\end{array}$ & $\begin{array}{c}\text { Charge } \\
\text { Density }\end{array}$ & $\begin{array}{c}\text { Equivalent } \\
\text { Thickness }\end{array}$ & $\begin{array}{c}\text { Relative Raman } \\
\text { Intensity }\end{array}$ \\
\hline \hline A & C/cm ${ }^{2}$ & nm & \\
\hline B & 0.000 & 0.0 & 1.00 \\
\hline C & 0.025 & 9.2 & 0.78 \\
\hline D & 0.061 & 22.6 & 0.66 \\
\hline E & 0.105 & 38.4 & 0.59 \\
\hline F & 0.206 & 55.9 & 0.54 \\
\hline
\end{tabular}

Table 2-1: Experimental results that accompany the Raman spectra in figure 2-21. (800 Series) 


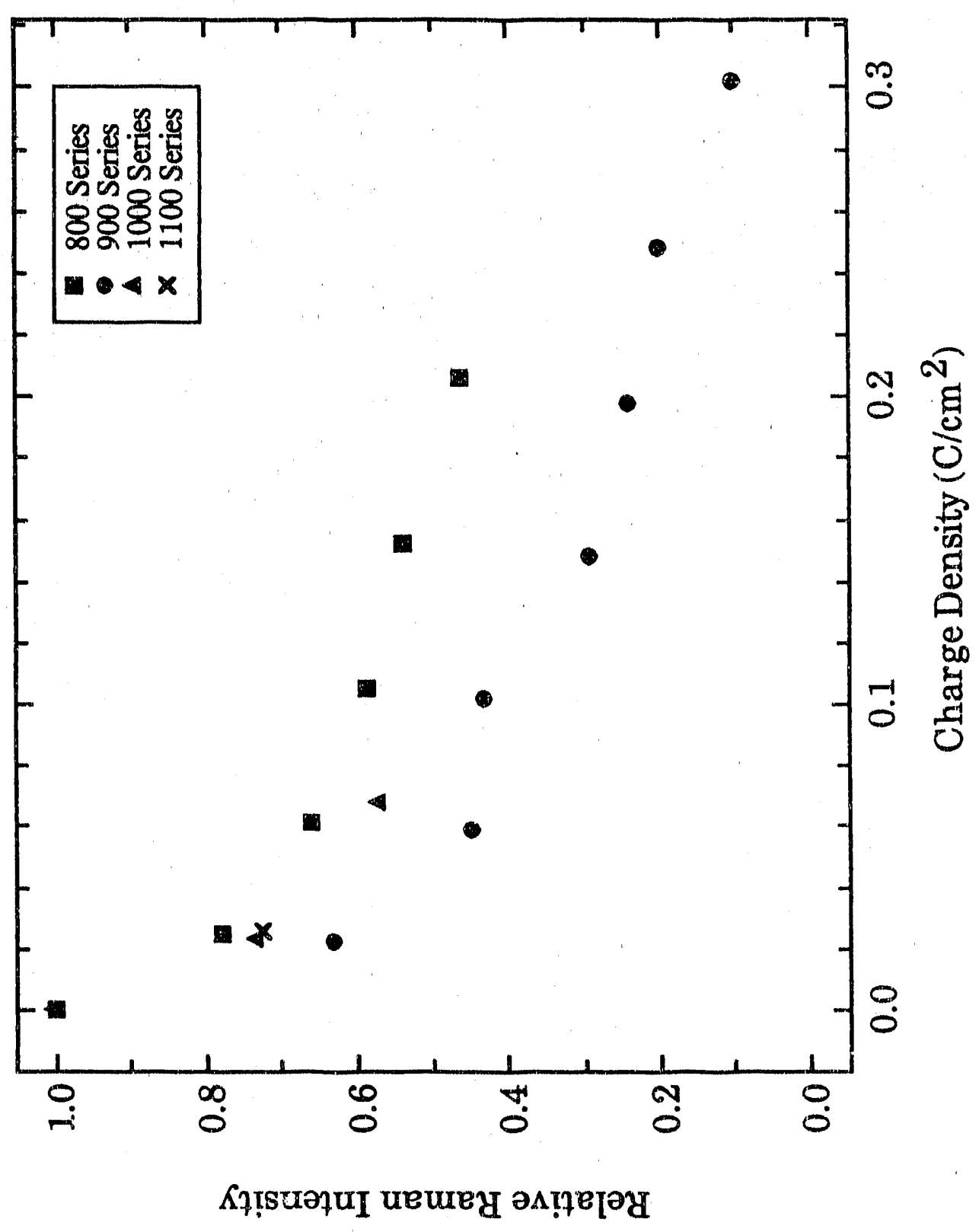

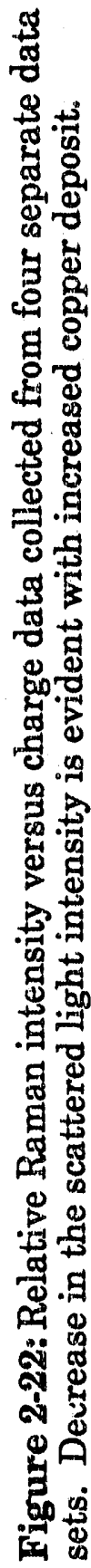




\section{Raman Models:}

Three optical models were developed to predict the attenuation of the Raman intensity from the graphite substrate by a nodular deposit. Assumptions common to all include $100 \%$ charge efficiency and spherical nodule shape. The models were constructed in collaboration with Robert Crocker.

The Raman scattering strength is proportional to the strength of the electric field transmitted into the graphite. The proportionality constant is the Raman scattering coefficient 23 :

$$
\mathrm{E}_{\mathrm{scat}, \mathrm{H}}=\alpha \mathrm{E}_{\mathrm{inc}, \mathrm{H}}
$$

whes e: $\mathrm{E}_{\text {Bcat, } \mathrm{H}}=$ amplitude of the scattered electric field from the HOPG

$E_{\text {inc }, \mathrm{H}}=$ amplitude of incident electric field within the HOPG

$\alpha=$ Raman scattering coefficient

For the copper covered surface, the amplitude of the electric field reaching the HOPG is determined by the transmission through the nodular copper layer.

$$
E_{\text {inc,H }}=t_{\text {eff,inc }} E_{\text {inc }}
$$

where: $E_{\text {inc }}=$ amplitude of the incident electric field on the copper nodule layer

$t_{\text {eff,ine }}=$ overall effective transmission coefficient for incident light through the copper film

The scattered light from the graphite substrate through the nodule layer is described by a similar expression for the amplitude of its electric field. The transmission coefficients for the incident and the scattered light are different, because of the different angles and wavelengths for the incident and scattered light. 


$$
\mathrm{E}_{\text {scat }, \mathrm{Cu}}=t_{\text {eff, }, \text { ccat }} \mathrm{E}_{\mathrm{Bcat}, \mathrm{H}}
$$

where: $E_{s c a t, C u}=$ amplitude of electric field of scattered light from a copper covered graphite surface $t_{\text {fff, scat }}=$ overall effective transmission coefficient for scattered light out through the copper film

Combining equations [2-17], [2-18] and [2-19] we obtain for the amplitude of the scattered electric field from copper covered graphite:

$$
E_{\text {scat }, C u}=\alpha t_{\text {eff, inc }} t_{\text {eff, scat }} E_{\text {inc }}
$$

A similar expression can also be derived for a bare HOPG surface:

$$
E_{\text {scat,bare }}=\alpha t_{\text {bare,inc }} t_{\text {bare,scat }} E_{\text {inc }}
$$

where: $E_{\mathrm{gcat}, \text { bare }}=$ amplitude of electric field of scattered light from a bare graphite surface $t_{\text {bare, inc }}=$ transmission coefficient for incident light through electrolyte/HOPG interface $t_{\text {bare, }, \text { cat }}=$ transmission coefficient for scattered light through HOPG/electrolyte interface

The relative Raman intensity is defined as the intensity of scattered light from a copper covered graphite surfore relative to that of the bare graphite surface. It ranges from 0.0 for compiete coverage by an opaque copper film to 1.0 for bare graphite. Intensity is related to the electric field by the simple relationship:

$$
\operatorname{Re} 1 \mathrm{I}=\frac{I_{\mathrm{Cu}}}{I_{\text {bare }}}=\frac{\left|\mathrm{E}_{\text {scat,Cu}}\right|^{2}}{\left|\mathrm{E}_{\text {scat, ware }}\right|^{2}}
$$

where: Rel $I=$ relative Raman intensity

$\mathrm{I}_{\mathrm{Cu}}=$ intensity of scattered light from copper covered graphite 


$$
I_{\text {bare }}=\text { intensity of scattered light from bare graphite }
$$

Substituting equations [2-20] and [2-21] into equation [2-22]:

$$
\operatorname{Rel} I=\frac{t_{\text {eff,inc }}^{2} t_{\text {eff,scat }}^{2}}{t_{\text {bare,inc }}^{2} t_{\text {bare,scat }}^{2}}
$$

Three different film models were developed and compared to the experimental measurements of Raman intensity versus charge. The three models include a simple geometric model, an effective medium approximation and a island model. (figure 2-23) The principal difference between the three models is the determination of the effective transmission coefficients.

\section{Physical}
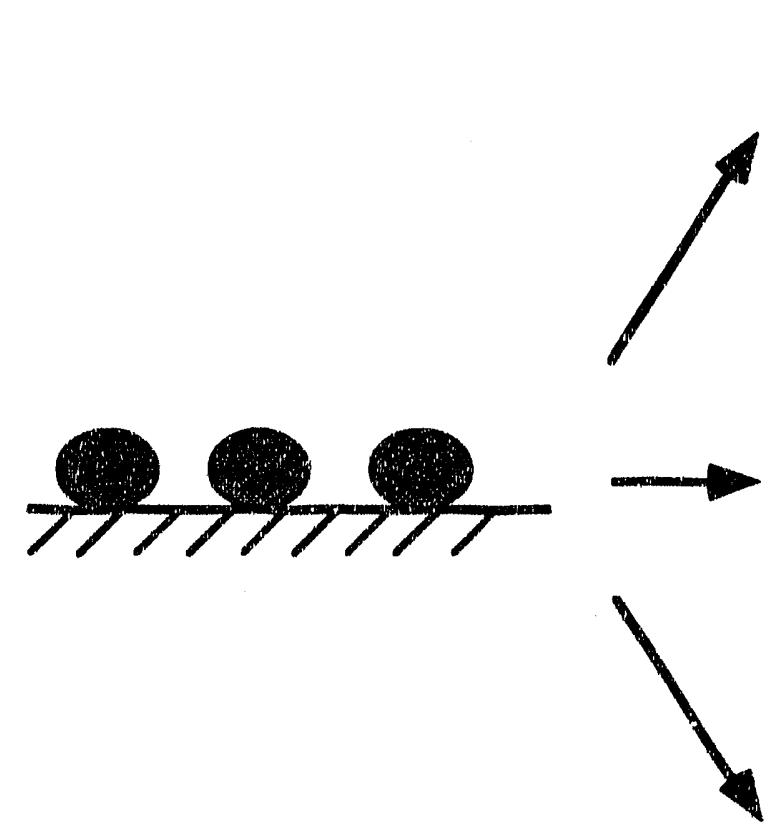

Optical Models
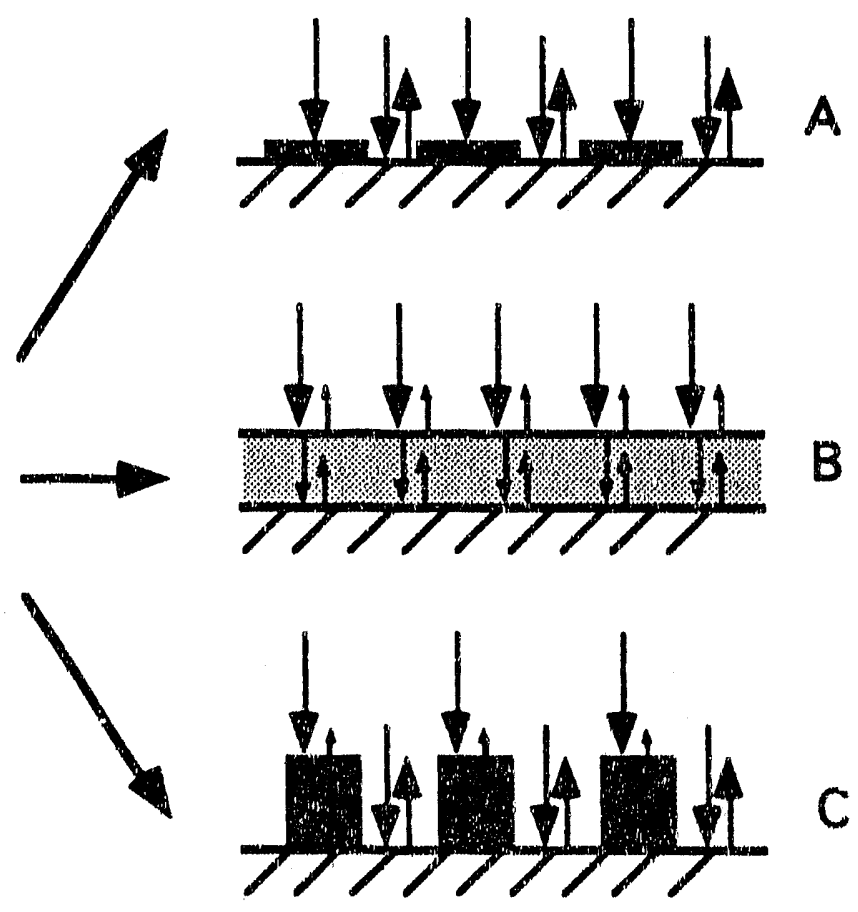

C

Figure 2-23: Schematic of optical models. A. Simple geometric model, B. Effective Medium Approximation, C. Island Model 


\section{Simple Geometric Model:}

The simplest optical model assumes the copper nodules block out the light, therefore the incident light reaches only a fraction of the graphite surface. The excluded areas are calculated as the projected area of the copper nodules. (figure 2-23A) The coverage is calculated to:

$$
\Theta=N \pi r^{2}
$$

where: $\Theta=$ fraction of surface covered by copper:

$\mathrm{N}=$ number density of nodules (nodule $/ \mathrm{cm}^{2}$ )

$\mathrm{r}=$ radius of a nodule $(\mathrm{cm})$

Assuming that copper is opaque, the transmittance of the incident light intensity through the nodules is zero. The effective transmittance through the copper layer is:

$$
T_{\text {eff,inc }}=(1-\theta) T_{\text {bare,inc }}
$$

where: Teff,inc $=$ Effective transmittance for incident light intensity in through the copper layer

Tbare, inc $=$ Transmittance for incident light intensity in through bare graphite/electrolyte interface

Since the transmittance of light intensity is the squared of the transmission coefficient of the amplitude of the electric field $\left(T=t^{2}\right)$, equation $[2=25 \mathrm{a}]$ and $[2-25 \mathrm{~b}]$ are equivalent.

$$
t_{\text {eff,inc }}^{2}=(1-\theta) t_{\text {bare, inc }}^{2}
$$

The expression for the effective transmittance of the scattered light out through the nodular layer is similar to equation [2-25]. Substituting into [2-23] results in equation [2-26] for the relative Raman intensity. 


$$
\operatorname{Re} I I=\frac{I_{C u}}{I_{\text {bare }}}=(1-\theta)^{2}
$$

Using the coverage determined by the Raman intensity and the measured total charge, the radius of the nodules and their number density can be calculated. The coverage, $\theta$, and the total charge, Qtotal, are both related to the nodule number density and the radius of the nodules, as shown below. The total charge can be expressed as:

$$
Q_{\text {total }}=N q_{\text {nodule }}
$$

where: $Q_{\text {total }}=$ charge density to entire surface $\left(\right.$ Coulombs $\left./ \mathrm{cm}^{2}\right)$ $q_{\text {nodule }}=$ charge to form single nodule (Coulomb/nodule)

Qnodule is expressed as:

$$
q_{\text {nodule }}=\frac{z F \rho}{M_{w}} v
$$

where: $\mathbf{z F}=$ Charge per mole of depositing species

$$
\text { ( } 2 \times 96487 \mathrm{C} / \mathrm{mol} \text { ) }
$$

$\rho=$ Density of metal deposit $\left(8.96 \mathrm{~g} / \mathrm{cm}^{3}\right)$

$\mathrm{M}_{\mathrm{W}}=$ Molecular weight of deposit $(63.5 \mathrm{~g} / \mathrm{mol})$

$\mathrm{V}=$ Volume of nodule $\left(\mathrm{cm}^{3}\right)$

Assuming a spherical nodule the volume is:

$$
V=\frac{4}{3} \pi r^{3}
$$

Combining equations [2-27] $[2-28]_{\text {, }}$ and [2-29] we obtain:

$$
Q_{\text {total }}=N \frac{4}{3} \pi r^{3} \frac{z F \rho}{M_{w}}
$$


The equation for the simple geometric coverage [2-24] and the total charge equation [2-30], can be easily solved for the number density, $N$, and the radius of the nodules, $r$. The results from this model for the set of experiments plotted in figure 2-21 are summarized in table 2-2. The nucleation density and radius results from this model are included along with number densities measured with the SEM at the end of the electrodeposition cycles.

\begin{tabular}{|c|c|c|c|c|}
\hline $\begin{array}{c}\text { Charge } \\
\text { Density }\end{array}$ & $\begin{array}{c}\text { Relative } \\
\text { Raman } \\
\text { Intensity }\end{array}$ & $\begin{array}{c}\text { Calculated } \\
\text { Nodule Radius }\end{array}$ & Coverage & $\begin{array}{c}\text { Calculated } \\
\text { Nucleation } \\
\text { Density }\end{array}$ \\
\hline$\left(\mathrm{C} / \mathrm{cm}^{2}\right)$ & $(\mathrm{nm})$ & - & 0.000 & $\left(\mathrm{~cm}^{-2}\right)$ \\
\hline 0.0000 & 1.000 & 59 & 0.116 & $1.06 \times 10^{9}$ \\
\hline 0.0250 & 0.781 & 90 & 0.188 & $7.42 \times 10^{8}$ \\
\hline 0.0614 & 0.659 & 123 & 0.235 & $4.98 \times 10^{8}$ \\
\hline 0.1047 & 0.585 & 157 & 0.267 & $3.45 \times 10^{8}$ \\
\hline 0.1523 & 0.537 & 178 & 0.320 & $3.22 \times 10^{8}$ \\
\hline 0.2062 & 0.463 & & & \\
\hline
\end{tabular}

Table 2-2: Calculated values for the nucleation density and coverage based on the siraple geometric model (Figure 2-23A) derived from the measured charge density and relative Raman intensity values reported in table 2-1. The calculated nucleation density values after each deposition step are also reported. Copper was deposited using a double pulse technique (Figure 2-20) from $5 \mathrm{mM} \mathrm{CuSO}_{4}$ and $1 \mathrm{M}$ aqueous $\mathrm{H}_{2} \mathrm{SO}_{4}$ elertrolyte. The nucleation density after the last deposition agrees well with the SEM measured nucleation density of $3.7 \times 10^{8} / \mathrm{cm}^{2}$. ( 800 Series)

The data in table 2-2 are from the experiment that gives the closest agreement to the simple geometric model for nucleation density. The agreement between calculated and other measured data sets is not as satisfactory. Table 2-3 summarizes the results of all of our experimental data sets. The predicted nucleation densities from the simple geometrical model are compared with measured SEM nucleation densities. For a majority of the experiments, the model predicts a higher nucleation density than the value observed by SEM. The model 
underestimates the ability of light to penetrate the nodular layer; it assumes that the nodules block out all of the light in their projected area. However, because the scattered light originates from within a finite penetration depth in the graphite beyond the surface, tight diffracted around the nodules scatter as well.

\begin{tabular}{|c|c|c|c|c|c|}
\hline $\begin{array}{c}\text { Sexies } \\
\text { Identification }\end{array}$ & $\begin{array}{c}\text { Charge } \\
\text { Density }\end{array}$ & $\begin{array}{c}\text { Relative } \\
\text { Raman }\end{array}$ & Coverage & $\begin{array}{c}\text { Calculated } \\
\text { Nucleation } \\
\text { Density }\end{array}$ & $\begin{array}{c}\text { SEM Measured } \\
\text { Nucleation } \\
\text { Denssity }\end{array}$ \\
\hline 800 & 0.2062 & 0.463 & 0.320 & $3.20 \times 10^{8}$ & $3.7 \times 10^{8}$ \\
\hline 900 & 0.3018 & 0.100 & 0.684 & $1.47 \times 10^{9}$ & $9.5 \times 10^{8}$ \\
\hline 1000 & 0.0678 & 0.567 & 0.247 & $1.38 \times 10^{9}$ & $9.6 \times 10^{8}$ \\
\hline 1100 & 0.0259 & 0.725 & 0.149 & $2.05 \times 10^{9}$ & $1.2 \times 10^{9}$ \\
\hline
\end{tabular}

Table 2-3: Measured and calculated nucleation densities after the last copper deposition step. The calculated nucleation densities were determined based on the simple geometric model. Copper was deposited using a double pulse technique (Figure 2-20) from $5 \mathrm{mM} \mathrm{CuSO}_{4}$ and $1 \mathrm{M}$ aqueous $\mathrm{H}_{2} \mathrm{SO}_{4}$ electrolyte.

There appears to be a linear relationship between the calculated and measured nucleation densities (Figure 2-24): the calculated nucleation densities are too large by approximately a factor of two. This linear relationship indicates that the nodule geometry may be other than spherical. The calculation was repeated for hemispherical geometry. The calculated nucleation densities based on hemispherical geometry are a factor of two too small. This leads to the conclusion that the nodules are truncated spheres, with volumes between thoose of a sphere and a hemisphere. 


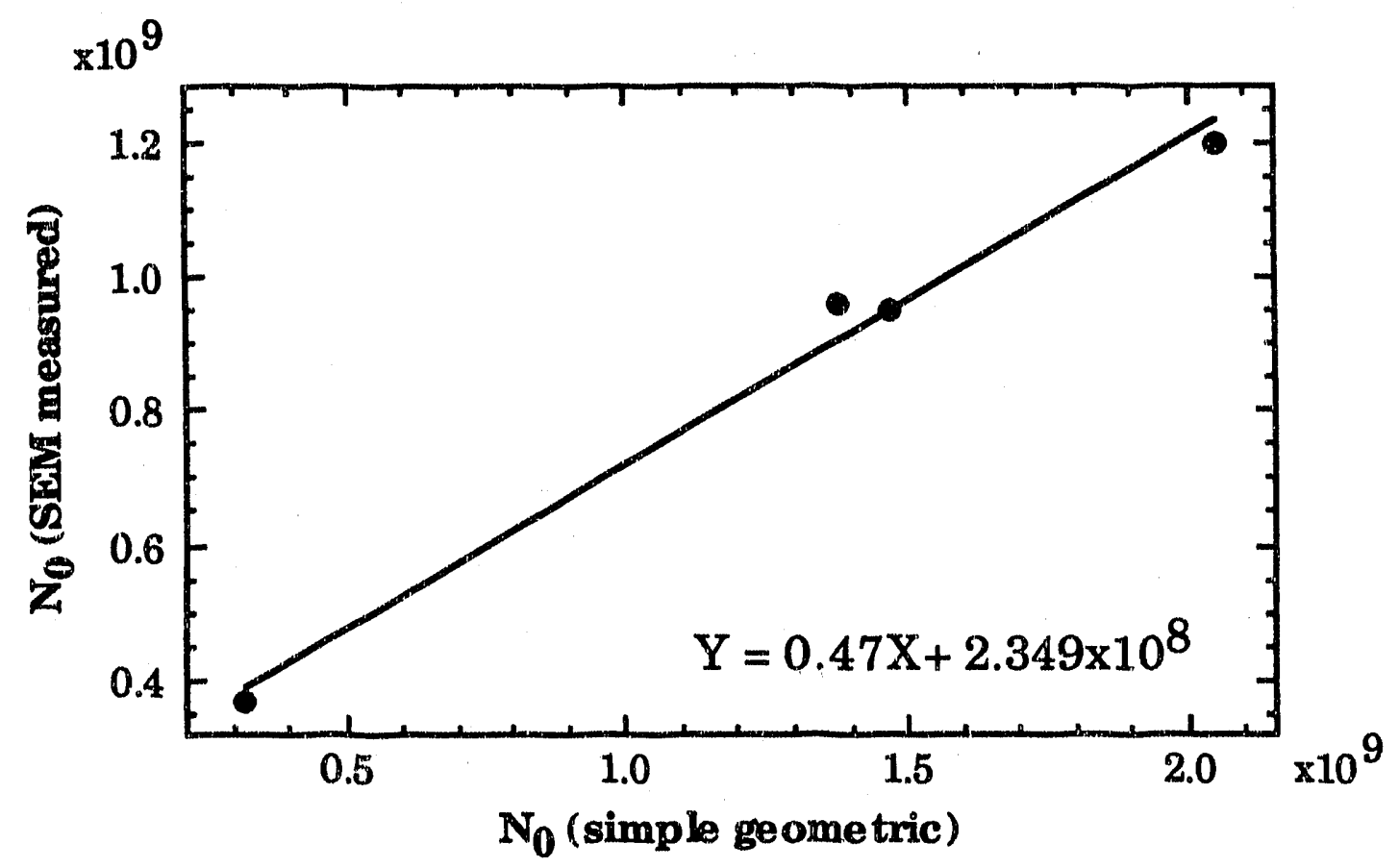

Figure 2-24: There is a linear relationship between the nucleation densities determined by SEM and those calculated from Raman spectroscopy based on the simple geometric model. (Data same as reported in table 2-3)

\section{Effective Film Modelis:}

To overcome the limitations of the simple geometric model, more detailed expressions for the transmission coefficient of the nodular layer were developed. The two models are: an effective medium approximation (EMA) and an island film model.

To determine the transmission coefficients through the different layers, the angle of propagation of the incident and the scattered light in each layer must be calculated. This can be accomplished using Snell's Law [2-31] along with the trigonometric identity [2-32], resulting in equation [2-33] for the angle in layer 1. 


$$
\mathrm{n}_{0} \sin \phi_{0}=\mathrm{n}_{1} \sin \phi_{1}=\mathrm{n}_{2} \sin \phi_{2}
$$

where: $\mathrm{n}_{0}=$ refractive index of electrolyte

$\mathrm{n}_{1}=$ complex refractive index of copper film

$\mathrm{n}_{2}=$ complex refractive index of graphite ${ }^{24}$

$\phi_{0}=$ propagation angle in electrolyte

$\phi_{1}=$ complex propagation angle in copper film

$\phi_{2}=$ complex propagation angle in graphite layer

$$
\begin{aligned}
& \sin ^{2} \phi+\cos ^{2} \phi=1 \\
& \cos \phi_{1}=\left[1-\left(\frac{n_{0} \sin \phi_{0}}{n_{1}}\right)^{2}\right]^{1 / 2}
\end{aligned}
$$

A similar equation for the angle in graphite medium, $\phi_{2}$, can be calculated by substituting the refractive index for layer $2, n_{2}$, in place of $n_{1}$ in equation [2-33]. Because the incidence angle is normal to the surface, the angle of incidence in both the copper film and the graphite is equal to the overall angle of incidence, which is $0^{\circ}$. For the $45^{\circ}$ scattered angle these values must be calculated for each layer. In absorbing media, such as copper or graphite, the propagation angle is represented by a complex number.

The transmission coefficients for each interface are calculated using the Fresnel equation. For s-polarization collected in our experiments, the transmission coefficient is equation [2-34].

$$
t_{12}=\frac{2 n_{1} \cos \phi_{1}}{n_{1} \cos \phi_{1}+n_{2} \cos \phi_{2}}
$$

where: $t_{12}=$ Fresnel transmission coefficient for incident light through the interface between layers 1 and 2 for s polarization. 
Each of the transmission coefficients, $t_{01}, t_{12}, t_{02}\left(=t_{\text {bare, }},{ }\right), t_{21}, t_{10}$, and $t_{20}$ (= tbare,scat) is calculated similarly. The subscripted numbers on the transmission coefficients indicate the direction of light as well as the location of the interface.

Figure 2-25 is a schematic showing the different refractive indices and the reflection and transmission coefficients.

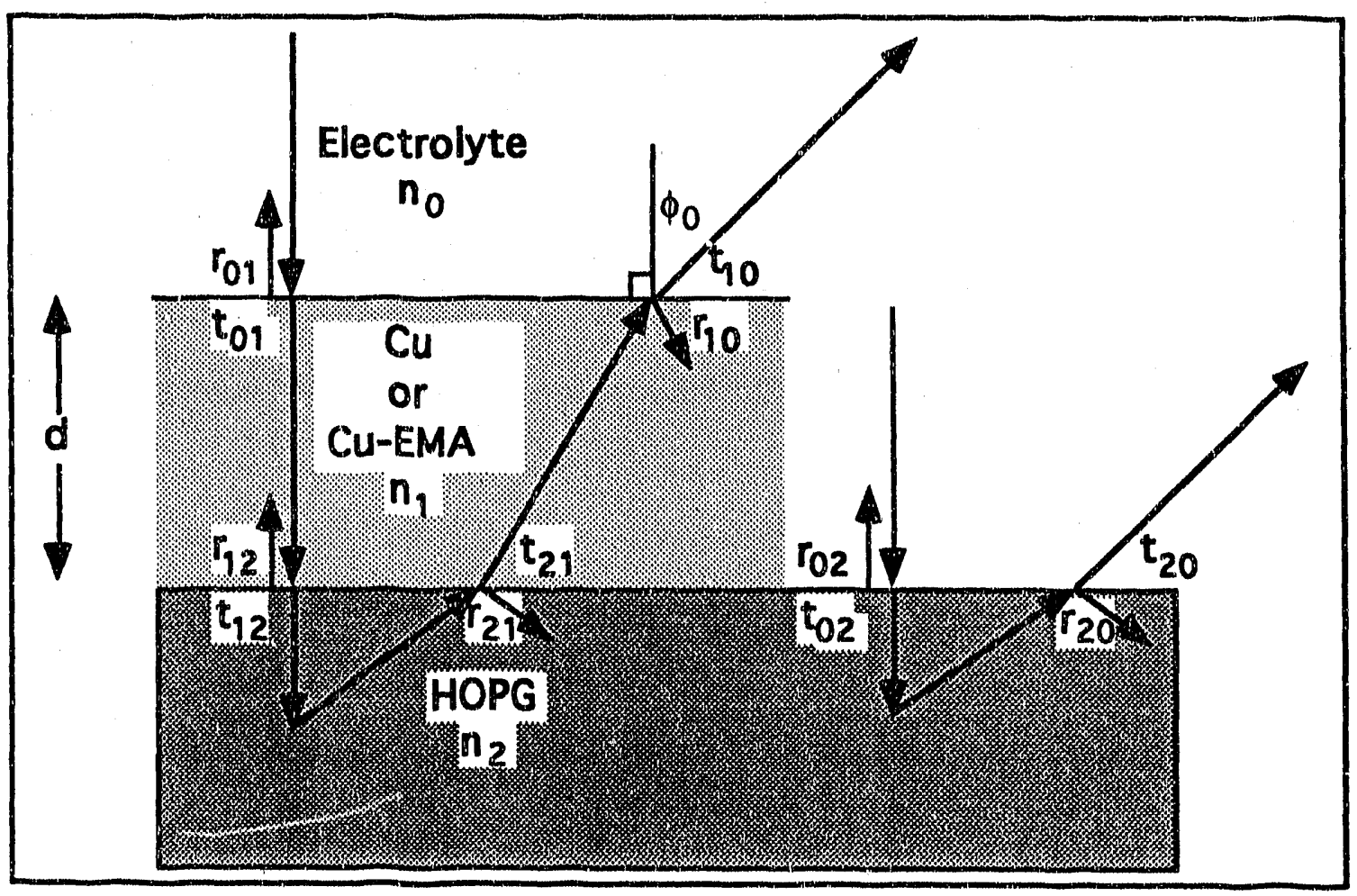

Figure 2-25: Schematic representation of refractive indices, transmission and reflection coefficients, angles of incidence and scatter, and the thickness of the copper layer. Subscripted numbers indicate materials and direction of light.

The interference effects through the copper film is calculated using the Drude equation, [2-35]. 


$$
t_{\text {drude, inc }}=\frac{t_{01} t_{12} \exp \left(-\frac{1}{2} \mathrm{iD}\right)}{1+\mathrm{r}_{01} \mathrm{r}_{12} \exp (-\mathrm{iD})}
$$

where: $t_{d r u d e, \text { inc }}=$ total transmission coefficient through the copper film for incident light, including thin film interference

$r_{01}=$ Fresnel reflection coefficient for s-polarized incident light at the interface between electrolyte and the copper film

$r_{12}=$ Fresnel reflection coefficient for s-polarized incident light at the interface between the copper film and graphite

and:

$$
D=\frac{4 \pi}{\lambda_{\text {inc }}} d n_{1} \cos \phi_{1}
$$

where: $\lambda_{\text {inc }}=$ wavelength of incident light

$d=$ thickness of copper film

The reflection coefficients for s-polarization are determined using the Fresnel equations for reflection [2-37].

$$
r_{12}=\frac{n_{1} \cos \phi_{1}-n_{2} \cos \phi_{2}}{n_{1} \cos \phi_{1}+n_{2} \cos \phi_{2}}
$$

where: $r_{12}=$ Fresnel reflection coefficient at the interface between the copper filia and graphite for s-polarized light.

The reflection coefficients at the two interfaces (electrolyte/copper and copper/graphite) are calculated similarly for the incident and scattered light. When cal ulating the Drude equation for the scattered light the corresponding reflection coefficients, $r_{21}$ and $r_{10}$ are used along with the wavelength of scattered light, $\lambda_{\text {scat }}$. 


\section{Effective Medium Approximation Model:}

In the effective medium approximation model ${ }^{25}$, the copper nodule layer is approximated by a homogeneous, effective medium. (figure 2-23B) This effective medium has a refractive index obtained of the refractive indices of the two constituent materials, the electrolyte and copper. The effective refractive index of the film is a weighted mixture of the two according to the Bruggeman rule. ${ }^{25}[2-38]$

$$
\sum_{i} \theta_{i} \frac{n_{i}^{2}-n_{\text {eff }}^{2}}{n_{i}^{2}+m_{\text {eff }}^{2}}=0
$$

where: $\theta_{i}=$ Volume fraction of species $i$ in the copper film $n_{i}=$ complex refractive index of species $i$ in the copper film

$n_{\text {eff }}=$ complex refractive index of the effective medium $\gamma \equiv 2$, screening factor related to symmetry

To determine the volume fraction of copper in the effective optical film, the thickness of the copper film is assumed to be equal to the radius of the actual copper nodules. Using the measured values for the nucleation density and total charge, the radius of the nodules is simple to calculate using the mass balance relationship in equation [2-30]. Equation [2-38] is then solved for the effective refractive index, $n_{\mathrm{eff}}$. This refractive index is substituted for $n_{1}$, from the general discussion of effective film models. $\left(n_{1}=n_{\text {eff }}\right)$ The calculated $t_{d r u d e, \text { inc }}$ and tdrude,scat $u$ sing this refractive index, become the $t_{\text {eff, inc }}$ and $t_{\text {eff,scat. }}\left(t_{\text {eff,inc }}=t_{d r u d e, \text { inc }}\right.$ and $\left.t_{\text {eff, scat }}=t_{d r u d e, s c a t}\right)$ Solving equation [2-23] leads to the determination of the relative Raman intensity.

Unfortunately, this model did not give satisfactory results. The calculated Raman intensities were below the measured values by orders of magnitude. 


\section{Island Model:}

In the island model, the effective film is assumed to be composed of cylindrical copper islands. (figure 2-23C) The transmission coefficient is calculated through the copper islands as well as through the bare graphite/electrolyte interface. To solve the island model, we assume the light that is transmitted through the copper nodules is coherently superimposed with the light that is transmitted through the bare graphite interface ${ }^{2}$. The assumption of coherent superposition allows us to add the amplitudes and the phases of the electric fields of the two light waves to determine the resulting electric field.

To calculate the transmission coefficients, instead of an effective refractive index for the copper film, the actual refractive index of copper is used. $\left(\mathrm{n}_{\mathrm{Cu}}=\mathrm{n}_{1}\right)$ $t_{d r u d e, i n c}$ and $t_{d r u d e, s c a t}$ are calculated using equation [2-35]. Thin film interference effects are accounted for in the copper islands. The coverage of copper islands over the surface is then calculated based on a mass balance equation similar to [2-24] and [2-30] but for cylindrical copper deposits. The expression connecting charge density and coverage becomes:

$$
\Theta=Q \frac{M_{w}}{z F p} d
$$

The overall effective transmittance of the light intensity for the copper film is calculated by taking weighted averages of the transmittance through the copper nodules and the transmittance through the bare graphite surface. The expression for the effective transmission coefficient, $t_{e f f, i n c}$, becomes:

$$
\begin{aligned}
& T_{\text {eff,inc }}=(1-\Theta) T_{\text {bare,inc }}+\Theta T_{\text {drude,inc }} \\
& t_{\text {eff,inc }}^{2}=(1-\Theta) t_{\text {bare,inc }}^{2}+\Theta t_{d r u d e, \text { inc }}^{2}
\end{aligned}
$$


$t_{\text {eff,scat }}$ is calculated similarly, using tbare,scat and $t_{\text {drude,scat. The relative Raman }}$ intensity is then calculated using equation [2-23].

Assuming the thickness is equal to the radius of the copper nodules, the results were encouraging, however, not completely satisfactory. Therefore, a better correlation between the thickness of the effective film, (d), and radius of the nodules, (r), on the surface was developed. When the effective film thicknesses were fitted to the measured relative Raman intensities, a linear relationship between the optical film thickness and the radius of the spherical copper nodules was observed. 2 he thickness is 1.15 times the radius.

Since the total amount of copper on the surface is not varied, changing the thickness of the effective film changes the copper coverage. Determining the film thickness empirically translates to determining an effective coverage.

The empirically determined effective optical film thickness was substituted into the model to make it useful as a predictive tool. The FORTRAN code written to solve for the relative Raman intensity is included in appendix $B$. The results for the same data set listed in table 2-1 are plotted in figure 2-26 along with measured Raman intensities. The calculated results agree well with the measured Raman intensities.

\section{Limitations of Raman as a Predictive Tool:}

To obtain spectra with definitive features, the scattered light had to be collected for a minimum of one to two minutes. Added to this duration is the time allowed for cooling the surface down, to avoid laser enhanced deposition. During this extended time, redistribution of copper between nodules is likely to be significant. The advantages of the in situ capalsilities of Raman are diminished by the possibility of affecting the surface during ana' ysis. 
More data for the empirically determined fit of the effective film thickness must be collected, before the developed model can be used reliably. Using the empirical relationship is a drawback of determining nucleation density with the island model. Developing a different model, which does not rely on an empirical relationship, may be necessary before the Raman intensity becomes a reliable tool in determining the nucleation density.

\subsection{Summary and Conclusions:}

The electrode surfaces were examined using both ex-situ and in-situ techniques to determine the nucleation density of the copper electrodeposit. $E x$-situ scanning electron microscopy was determined to be the most reliable method, compared with scanning tunneling microscopy and Raman spectroscopy, though both of these techniques were shown to work under special conditions and when great care is taken.

The bare graphite surfaces were imaged at atomic resolution using the STM in both air and water. However, when samples with copper deposits where studied, the images were very similar to images of bare graphite, with no indication of copper on the surface. A possible explanation for this is the poor adhesion of copper to the graphite substrate; because the nodules are not firmly attached to the surface, interaction with the probe tip can cause the nodules to move. At high coverages, when the nodules are densely packed and difficult to move out of the way, the copper nodules on the graphite were imaged with the STM. The results were comparable to the results from the SEM. The restriction to high coverage limits the value of the STM as a tool for determining nucleation densities. 

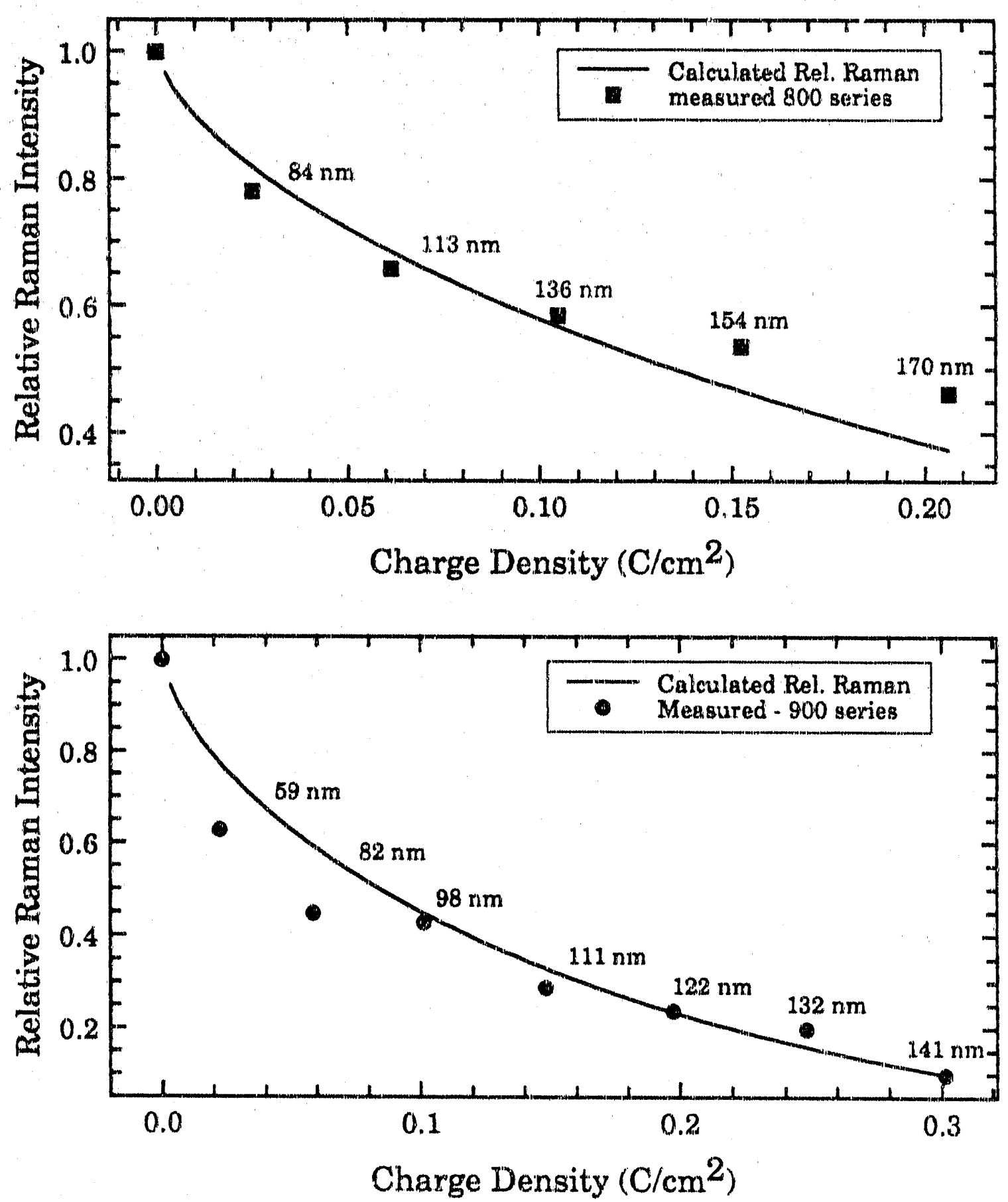

Figure 2-26: Comparison of the calculated and measured relative Raman intensities for two data sets.( 800 and 900 Series) Upper figure corresponds to data presented in table 2-1. The Raman intensities were measured using the island model, assuming constant nucleation density. 800 Series: $N_{0}=3.7 \times 10^{8} / \mathrm{cm}^{2}$, 900 Series:

$N_{0}=9.5 \times 10^{8} / \mathrm{cm}^{2}$. The calculated radii based on opherical nodules are reported. 
The relationship between copper coverage and the graphite Raman signal was established using Raman spertroscopy. With increased copper coverage, the intensity of the Raman scattered light decreases. Determining nucleation densities, from this decreased signal, however, wass difficult. Optical models, which were developed to determine the nucleation density from the attenuation of the graphite Raman intensity, had to rely on an empirical relationship in order to agree with the measured data. Other problems such as long data collection times and laser enhanced deposition, diminish the advantages of the in-situ capabilities of Raman due to the possibility of affecting the surface during analysis.

Material redistribution between nodules was a major concern, especially in ex-situ analysis. The differences in surface energies between the copper and the graphite results in small nodules dissolving, while the larger nodules grow. If the smaller nuclei disappear, the nucleation density determined may be too small. A cathodic potential during open-circuit was measured and is attributed to redistribution. To minimize errors in measured nucleation densities, the samples were removed from the electrolyte as quickly as possible after the completion of copper deposition.

The Scharifkex and Mostany ${ }^{3}$ model, for determination of nucleation density from the current transient to a potentiostatic step, was applied to electrodeposition of copper on HOPG. The model fits the experimentally measured current response to a potential step well. The model-predicted nucleation densities, however, are much lower than those measured with the SEM. Therefore this analysis could not be used to predict nucleation densities.

In the absence of a reliable in -situ method, ex-situ SEM imaging followed by direct counting of the number of copper nodules, is judged to be the best method for the determination of nucleation density. 


\section{References:}

1 M.s. Armstrong, Whe Role of Inhibitors During Electrodeposition of Thin Metallic Films", Ph.D. Thesis, Universicy of California, Lawrence Berkeley Laboratory, LBL-28972 (1990).

2 S.T. Mayer, "An In-Situ Study of the Anodic Film. Formation of Cu, Ag, and $\mathrm{Zn}$ in Alkaline Media", $\mathrm{Ph} . \mathrm{D}$. Thesis, University of California, Lawrence Berkeley Laboratory, LBL-28085 (1989).

3 B.R. Scharifker, J. Mostany, "Three-Dimensional Nucleation with Diffusion Controlled Growth, Part I. Number Density of Active Sites and Nucleation Rates per Site", J. Electroanal. Chem, 177, 13 (1984).

M. Fleischmann, H.R. Thirsk, "Metal Deposition and Electrocrystallization", in Advances in Electrochemistry and Electrochemical Engineering, Vol. 3, p.123-210, edited by P. Delahay and C.W. Tobias, Wiley, New York, 1963.

5 G. Binnig, H. Rohrer, Ch. Gerber, E. Weitsel, "Tunneling through a Controllable Vacuum Gap", Appl. Phys. Lett. 40, 1.78 (1981).

6 G. Binnig, H. Rohrer, Ch. Gerber, E. Weibel, "Surface Studies by Scanning Tunneling Microscopy", Phys. Rev. Lett. 49, 57 (1982).

7 G. Binnig, H. Rohrex, Ch. Gerber, E. Weibel, "7x77 Reconstruction on Si(111) Resoived in Real Space", Phys. Rev. Lett. 50, 120 (1983).

8 J. Schneir, R. Sonnenfeld, P.K. Hansma, J. Tersoff, "Tunneling Microscopy Study of the Graphite Surface in Air and Water", Phys. Rev. B 34, 4979 (1986).

9 G. Binnig and D.P.E. Smith, "Single-Tube Three Dimensional Scanner for Scanning Tumneling Microscopy", Rev. Sci. Instrum. 5\%, 1688 (1986).

10 M.J. Armstrong, "The Role of Inhibitors During Electrodeposition of Thin Metallic Films", Ph.D. Thesis, Appendix B, University of California, Lawrence Berkeley Laboratory, LBL-28972 (1990). 
11 H. Lomke, T. Göddenbenrich, H.P. Bochem, U. Hurtmann, C. Heiden, "Improved Microtips for Scanning Probe Microscopy", Rev. Sci. Instrum. 61, $2538(1.990)$.

12 P.K. Hansma, V.B. Elings, O. Marti, C.E. Bracker, "Scanning Tunneling Microscopy and Atomic Microscopy: Application to Biology and Technology", Science 242, 209 (1988).

13 G. Binnig, H. Fuchs, Ch. Gerber, H, Rohrer, E. Stoll, E. Tosatti。 "EnergyDependent State-Density Corrugation of a Graphite Surface as Seen by Scanning Tunneling Microscopy", Europhysics Lett. 1, 31 (1986).

14 S.I. Park, C.F. Quate, "Tunneling Microscopy of Graphite in Air", Appl. Phys. Lett. 48, 112 (1986).

15 R. Sonnenfeld, P.K. Hansma, "Atomic-Resolution Micmscopy in Water", Science 232, 211 (1986).

16 S. Eppell, G. Chottiner, D. Scherson, G. Pruett, "STM of Platinum Deposits on the Basal Plane of HOPG", Langmuir 6, 1316 (1990).

17 M. Szklarczyk, J.O'M. Bockris, "In-Situ STM Studies of Lead Electrodepasition on Graphite Substrate", J. Electrochem. Soc. 137, 452 (1990).

18 E. Gauz, K. Sattler, J. Clarke, "Scanning Tunneling Microscopy of $\mathrm{Cu}, \mathrm{Ag}$, $\mathrm{Au}$, and $\mathrm{Al}$ Adatoms, Small Clusters, and Islands on Graphite", Surface Science 219, 33 (1989).

19 H. Chang, A.J. Bard, "Observation and Characterization by Seanning Thunneling Microscopy of Structures Generated by Cleaving Highly Oriented Pyrolytic Graphite”, Langmuir 7, 1143 (1991).

20 K. Kinoshita, in Carbon: Electrochemical and Physicochemical Properties, p.118, Wiley, New York, 1988. 
21 R.J. Bowling, R.T. Packard, R.I. McCreery, "Activation of Highly Oriented Pyrolytic Graphite for Heterogeneous Electron 'Transfer: Relationship between Electrochemical Performance and Carbon Microstructure", J. Am. Chem. Soc. 111, 1217 (1989).

22 R.S. Robinson, "Morphology and Electrochemical Effects of Defects on Highly Oriented Pyrolytic Graphite», J. Electrochem. Soc. 138, 2412 (1991).

23 J.W. Ager III, D.K. Veirs, G.M. Rosenblatt, "Raman Intensities and Interference Effects for Thin Films Adsorbed on Metals", J. Chem. Phys. 92, 2067 (1990).

24 E.A. Taft, H.R. Philipp, "Optical Properties of Graphite”, Phys. Rev. 138, A197 (1965).

25 D.E. Aspnes, J.B. Theeten, "Investigation of EMA Models of Microscopic Roughness by Spectroscopic Ellipsometryn, Phys. Rev. B 20, 3292 (1979). 


\section{Chapter 3:}

\section{ELECTROCHEMICAL ANALYSIS}

\subsection{Cyclic Voltammetry:}

\section{Experimental Procedure:}

For quantitative characterization of the difference in the deposition behavior on low electron density substrates and on metal substrates, we compared the results from two cyclic voltammograms (CV), one on a platinum electrode and the other on highly oriented pyrolytic graphite (figures. 3-1 and 3-2). In cyclic voltammetry, potential is scanned while the current response is measured. The features of the current versus potential plot are indicative of the reactions on the surface.

In these experiments, the potential was scanned at the rate of $50 \mathrm{mV}$ per second in stagnant, $50 \mathrm{mM}$ copper sulfate, $1 \mathrm{M}$ aqueous sulfuric acid electrolyte. The potential was measured versus a copper reference electrode. The initial scan direction is cathodic beginning from zero potential. Both the platinum and the HOPG electrodes were placed horizontally, facing up parallel to the counter electrode.

\section{Comparison of Results:}

Examining the $\mathrm{CV}$ on platinum, (figure 3-1) at zero potential versus the copper reference there is no curreni. Scanning just negative of the zero mark, a cathodic current is detected. 'This is due to cupric ions $\left(\mathrm{Cu}^{++}\right)$reducing to copper metal. The current levels out when the electrolyte near the surface becomes depleted of $\mathrm{Cu}^{++}$ions. At this point, the current becomes mass transport limited. At more negative potentials hydrogen ion $\left(\mathrm{H}^{+}\right)$reduction begins, and the cathodic current once again increases. On the reverse scan, the anodic current increases at 


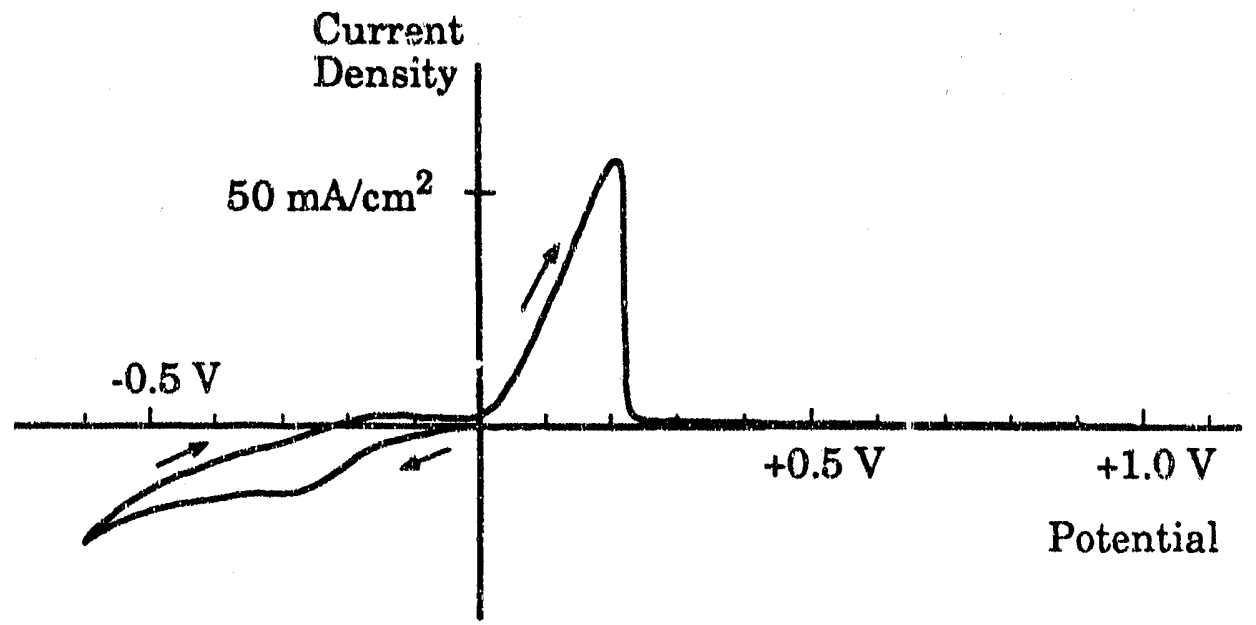

Figure 3-1: Cyclic voltammogram on platinum electrode from stagnant, aqueous electrolyte containing $50 \mathrm{mM} \mathrm{CuSO} 4$ and $1 \mathrm{M} \mathrm{H} 2 \mathrm{SO} 4.50 \mathrm{mV} / \mathrm{sec}$ scan between -0.6 and $+1.0 \mathrm{~V}$ versus a copper reference electrode.

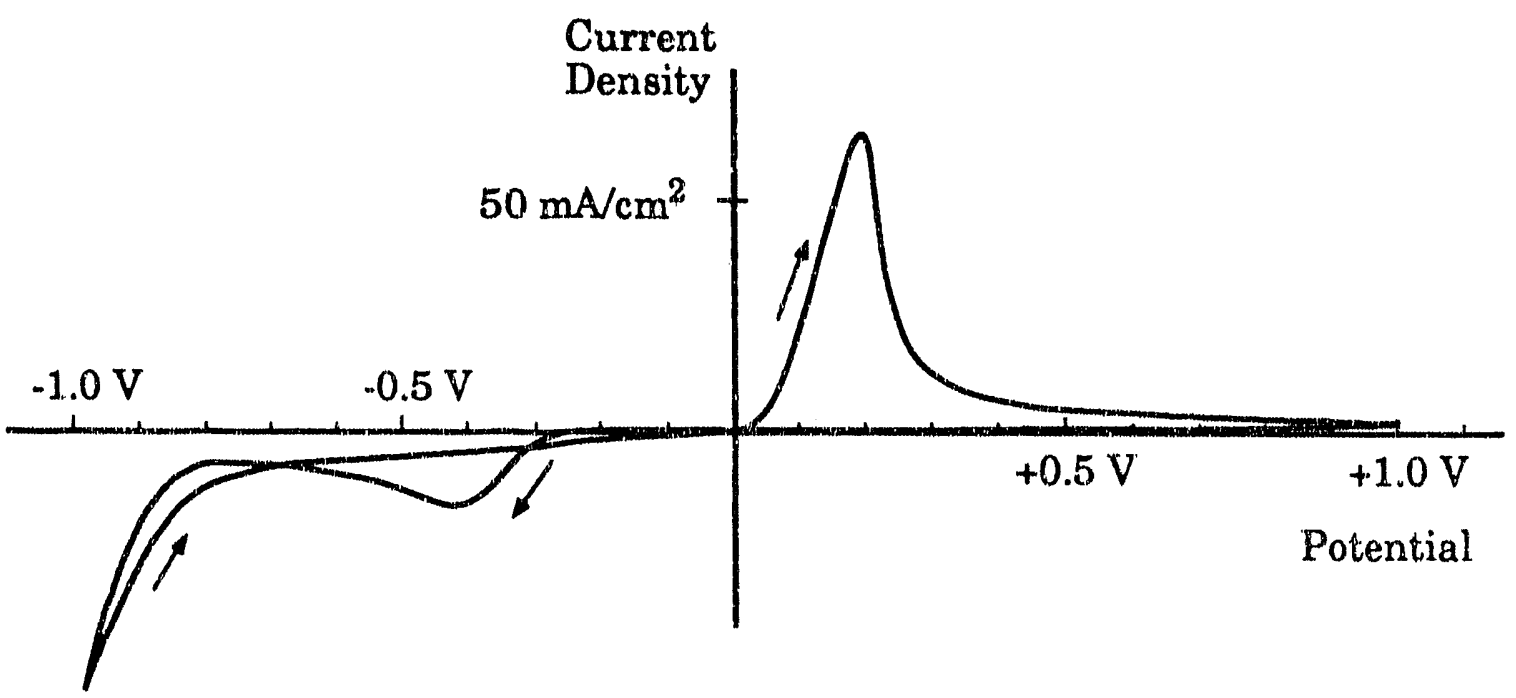

Figure 3-2: Cyclic voltammogram on HOPG electrode from stagnant, aqueous electrolyte containing $50 \mathrm{mM} \mathrm{CuSO} 4$ and $1 \mathrm{M} \mathrm{H}_{2} \mathrm{SO}_{4} .50 \mathrm{~m} \mathrm{~m}^{\mathrm{*}} / \mathrm{sec}$ scan between -1.0 and $+1.0 \mathrm{~V}$ versus a copper reference electrode. 
potentials just anodic of the zero mark. This current is due to the copper oxidation reaction to cupric ions.

On the HOPG electrode the situation is different. In this experiment, all of the conditions of electrolyte concentration and scan rate are identical to those in the runs with platinum. When a negative potential sweep is applied, a current response is not seen immediately (figure 3-2). Only at about $-250 \mathrm{mV}$ versus copper is the current from the copper reduction reaction detected. The hydrogen response is also shifted by $250 \mathrm{mV}$. In the reverse scan, as soon as an anodic potential is applied, the copper immediately begins to dissolve, as in figure 3-1. With repeated scans the same behavior persists.

The overpotential required to begin copper electrodeposition on HOPG is the nucleation overpotential for the system. This is the potential needed to nucleate on the graphite electrode. Comparing the two scans, one on platinum and the other on HOPG, the difference in nucleation overpotential is measured to be $250 \mathrm{mV}$.

If the potential is scanned only between $-1.0 \mathrm{~V}$ and $0.0 \mathrm{~V}$ (figure 3-3), and no anodic potential is applied, the first scan on HOPG is identical to the cathodic half of that shown in figure 3-2. Yet in the second scan, the nucleation overpotential disappears. This is as expected, because during the first scan copper is deposited on the graphite substrate, and because no anodic current is passed between the two scans the copper is not stripped off and it remains on the surface. During the second scan the copper deposition is taking place on the copper nodules that already exist on the surface, and a large nucleation overpotential is not expected on a copper substrate. The decrease in the copper deposition current is seen because the concentration of $\mathrm{Cu}^{++}$ions is depleted during the first scan. 
(A)

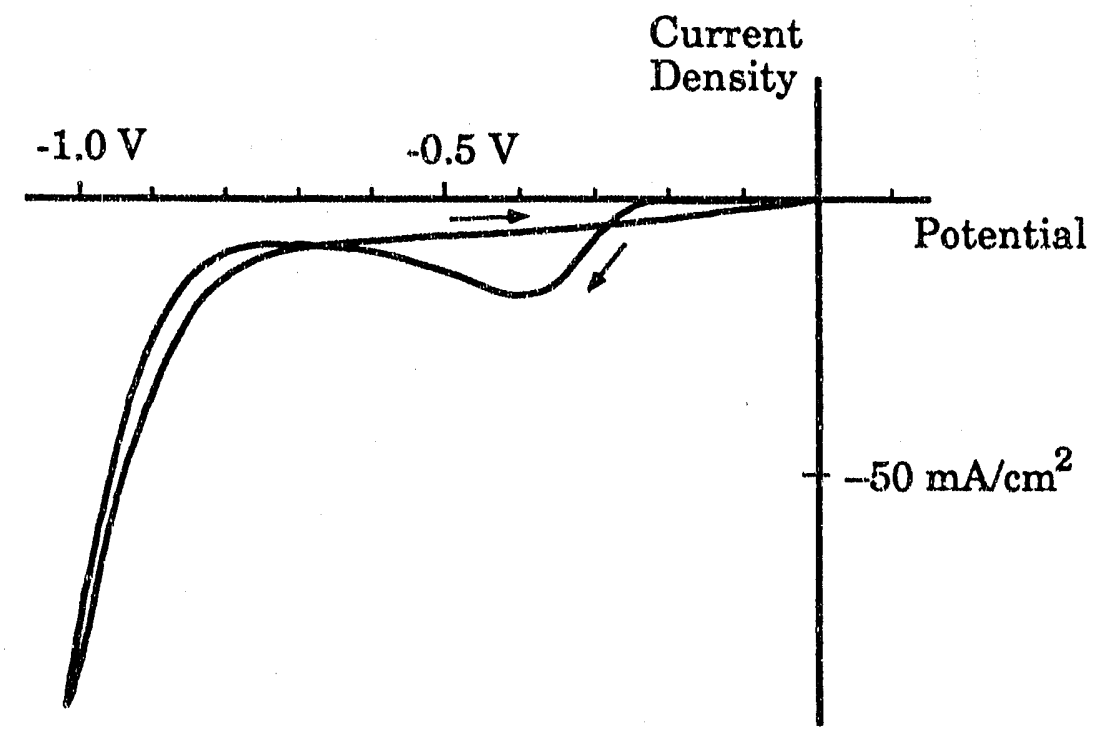

(B)

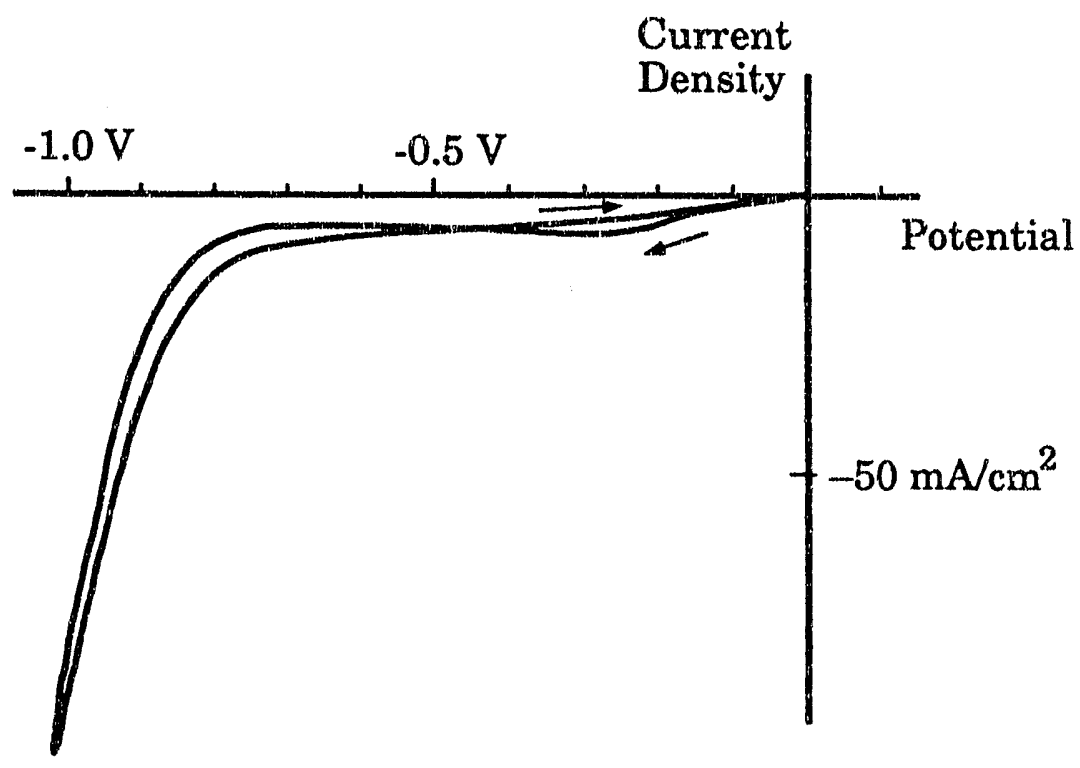

Figure 3-3: First (A) and second (B) sweeps of a CV on HOPG electrode from stagnant, aqueous electrolyte containing $50 \mathrm{mM} \mathrm{CuSO} 4$ and $1 \mathrm{M} \mathrm{H} 2 \mathrm{SO} 4.50 \mathrm{mV} / \mathrm{sec}$ scan between 0 and $-1.0 \mathrm{~V}$ versus a copper reference electrode. 


\subsection{Controlling and Increasing the Nucleation Density:}

To change the nucleation behavior, we must first understand the effect different variables have on the nucleation phenomena. The effects of the current, potential, repegted pulsing, concentration and inhibitors were studied. The results from these experiments follow.

\section{Effect of Current Density:}

In galvanostatic experiments, the desired current density is set externally and the potentiostat applies the required potential. A potential transient for such an experiment is reproduced in figure 3-4. This experiment was a galvanostatic step to $10 \mathrm{~mA}$ for one zecond. The area of the graphite substrate was $0.36 \mathrm{~cm}^{2}$, therefore the overall current density was $27.8 \mathrm{~mA} / \mathrm{cm}^{2}$. The potential is measured versus a copper reference electrode. The concentration of electrolyte is $50 \mathrm{mM} \mathrm{CuSO}_{4}$ and $1 \mathrm{M}$ aqueous $\mathrm{H}_{2} \mathrm{SO}_{4}$.

Initially, the potential rises very quickly to nucleate the surface with copper nodules. Once the surface is nucleated, growth is favored over continued nucleation, and the current flows to the existing nuclei. As the nodules grow, the surface area of metal increases. With the increased area, the overpotential decreases. As the concentration of copper ions decreases near the substrate, the current becomes mass transport limited and the potential increases until another reaction can sustain the required current, in this case, the reduction of hydrogen, which begins at about -1.0 $\mathrm{V}$ versus the copper reference electrode. The potential slowly drops to zero as the current is turned off and the concentration field relaxes. 


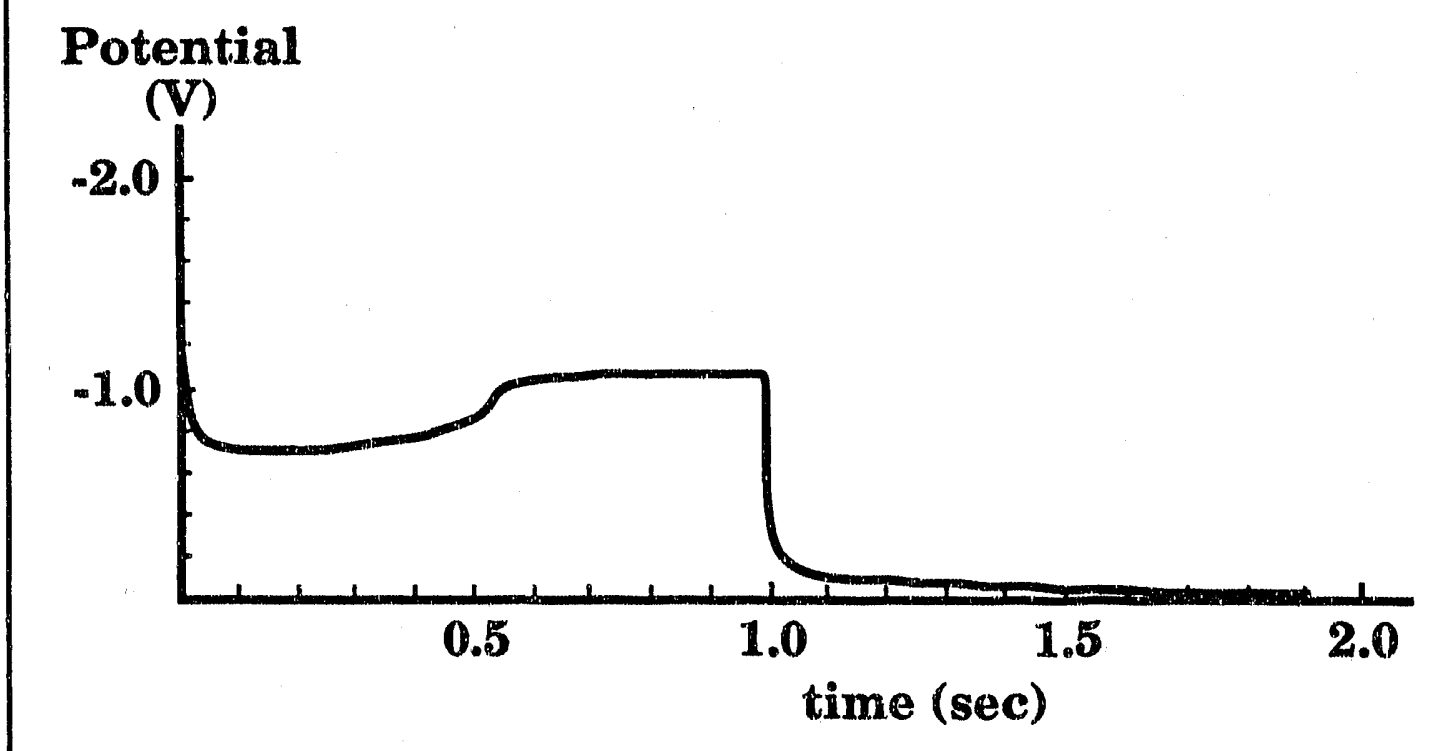

Figure 3-4: Potential transient for a galvanostatic step experiment. The current step was to $27.8 \mathrm{~mA}^{\mathrm{cm}} \mathrm{cm}^{2}$ for 1.0 sec, from $50 \mathrm{mM} \mathrm{CuSO}_{4}$ and $1 \mathrm{M} \mathrm{H}_{2} \mathrm{SO}_{4}$. The potential is measured versus a copper reference electrode.

A series of galvanostatic step experiments were performed to determine the effect of applied current on the nucleation density. Varying current densities in the range from $1.2 \mathrm{~mA} / \mathrm{cm}^{2}$ to $313 \mathrm{~mA} / \mathrm{cm}^{2}$ were studied. The total amount of charge passed was kept constant at $10 \mathrm{mC}$, to keep the total amount of copper on the surface constant in all the experiments. Because of slight differences in electrode sizes, the charge densities ranged between $27-35 \mathrm{mC} / \mathrm{cm}^{2}$. After the samples were prepared, they were rinsed and dried under a nitrogen stream. The nucleation. densities were then determined using the scanning electron microscope and counting the nodules that are imaged. Averages over many locations on the surface are reported in Table 3-1. Nodule radii ranged between 40 and 100 nanometers. Typical nucleation densities were on the order of $10^{8} \cdot 10^{9}$ nodules per $\mathrm{cm}^{2}$. 


\begin{tabular}{|c|c|c|}
\hline Current Density & Radius & Nucleation Density \\
\hline$\left(\mathrm{mA} / \mathrm{cm}^{2}\right)$ & $(\mathrm{nm})$ & $\left(\right.$ nuclei $\left./ \mathrm{cm}^{2}\right)$ \\
\hline 1.2 & 40 & $3 \times 10^{8}$ \\
\hline 5.1 & 55 & $2 \times 10^{8}$ \\
\hline 16.9 & 100 & $1 \times 10^{8}$ \\
\hline 27.8 & 52 & $3 \times 10^{9}$ \\
\hline 177 & 40 & $3 \times 10^{9}$ \\
\hline
\end{tabular}

Table 3-1: Results from galvanostatic step expeximents. The measured nodule radii and nucleation densities were determined using the SEM. Copper was deposited on to $\mathrm{HOPG}$ from $50 \mathrm{mM} \mathrm{CuSO}_{4}, 1 \mathrm{M} \mathrm{H}_{2} \mathrm{SO}_{4}$ aqueous electrolyte by means of galvanostatic step experiments.

The determination of the size of the nodules using the SEM is approximate. The apparent nodule size could be larger than the actual one because of charging of the copper deposit. The numbers are reported here for an approximate measure of size. Discerning a clear trend in the data is not possible. The nucleation density appears to be dropping with increased current density for the first few entries in the table, followed by a large increase for 27.8 and $177 \mathrm{~mA} / \mathrm{cm}^{2}$.

At higher current densities, hydrogen evolution causes damage to the surface of the graphite. (figure 3-5) In this image, the formerly atomically flat layers of graphite appear to be pulled away from the surface, after evolving hydrogen on the surface at $313 \mathrm{~mA} / \mathrm{cm}^{2}$.

Metal deposition is not uniform across the surface, but instead, is favored on the existing nodules. The overall current density is determined by dividing the total current by the surface area of graphite that is exposed, whereas the current only flows to the nodules, whose size is constantly changing. The microscopic current density on the nodules is different from the current density to the entire surface, and 
it is constantly decreasing as the nodules grow. To keep the driving force for metal deposition constant during the deposition period, potentiostatic experiments were performed.

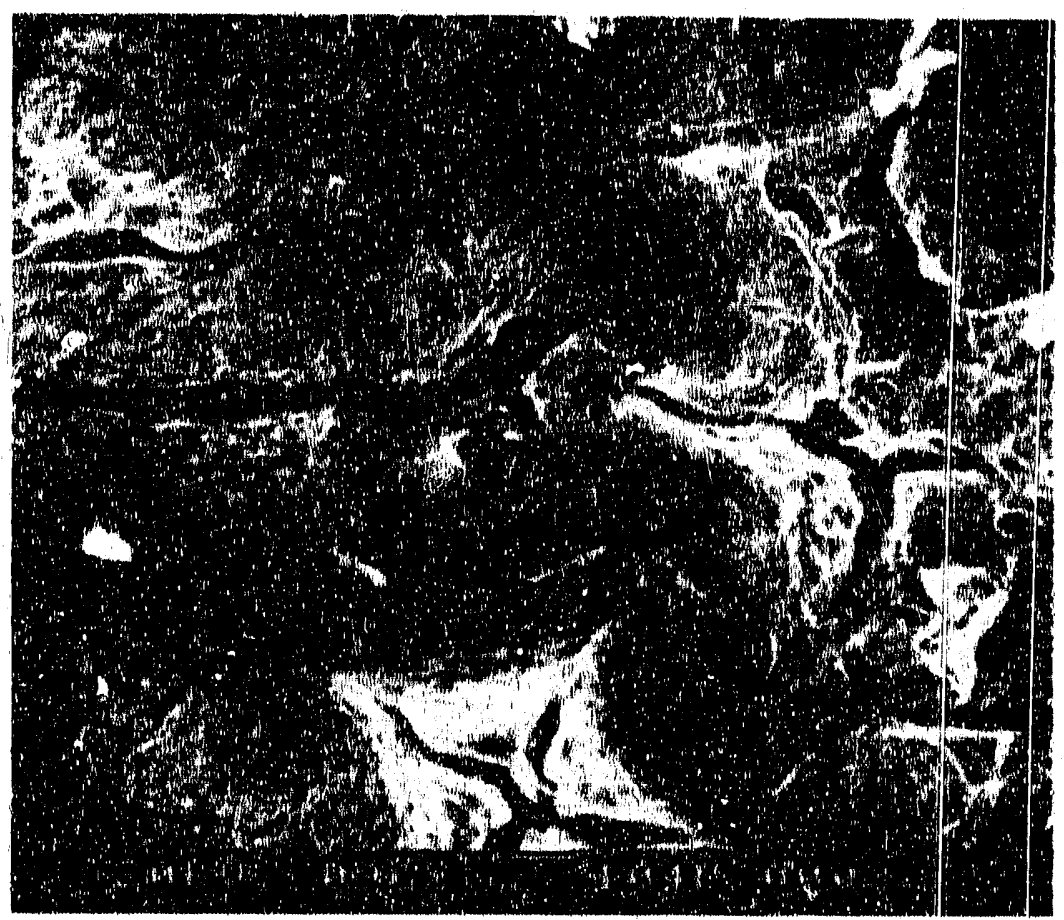

Figure 3.5: SEM image of disordered HOPG elestrode, due to hydrogen evolution at high current densities. Electrolyte: $50 \mathrm{mM} \mathrm{CuSO}_{4}$ and $1 \mathrm{M}^{2}$ aqueous $\mathrm{H}_{2} \mathrm{SO}_{4}$. Current density: $313 \mathrm{~mA} / \mathrm{cm}^{2}$.

\section{Effect of Potential:}

To study the effect of the applied driving force, potential step experiments were performed at different potentials for various durations. The samples were then rinsed in distilled water, dried under nitroger, and analyzed in the SEM. At high magnifications the nodules can be counted to determine nucleation densities. The values plotted are averages taken over nucleation densities determined at many different locations on the surface. Typically, the surface was analyzed in sixteen locations to eliminate bias due to spatial variations in nucleation density. 
The current transient for a typical potential step experiment is reproduced in figure 3-6. The current rises while the nodules are growing and more metal surface area is created. It eventually peaks and starts decreasing as the concentration of the $\mathrm{Cu}^{++}$ions drops near the surface. With the overlap of concentration fields around individual nodules, diffusion to the surface becomes one dimensional planar diffusion. The current continues to decrease with time, as the depletion zone increases.

The increase in applied potential shows a very strong effect on the nucleation density, which as shown in figure 3-7, rises steeply with increased overpotential. Since a higher patential equates to a higher driving force, this result is not unexpected. The experiments are potential steps in $50 \mathrm{~mm} \mathrm{CuSO} 4$ and $1 \mathrm{M} \mathrm{H}_{2} \mathrm{SO}_{4}$ electrolyte. The reference electrode is copper.

The potential step experiments had to be performod within a restricted window of potentials. At very low potentials, copper deposition does not occur because the potential falls below the nucleation overpotential. At potentials slightly higher, the nodules were too small for ex-situ. SEM analysis. Only at about 600-700 $\mathrm{mV}$ overporential were the nodules easily imaged by SEM. At higher potentials than those shown in figure 3-7, hydrogen reduction begins. Hydrogen evolution reduces the current efficiency making it difficult to determine the exact amount of copper deposited. A more important consideration than reduced current efficiency is the increased surface disorder on the HOPG caused by hydrogen evolution. Under extremely high potential conditions, the surface can be disordered to the extent shown in figure 3-5. 


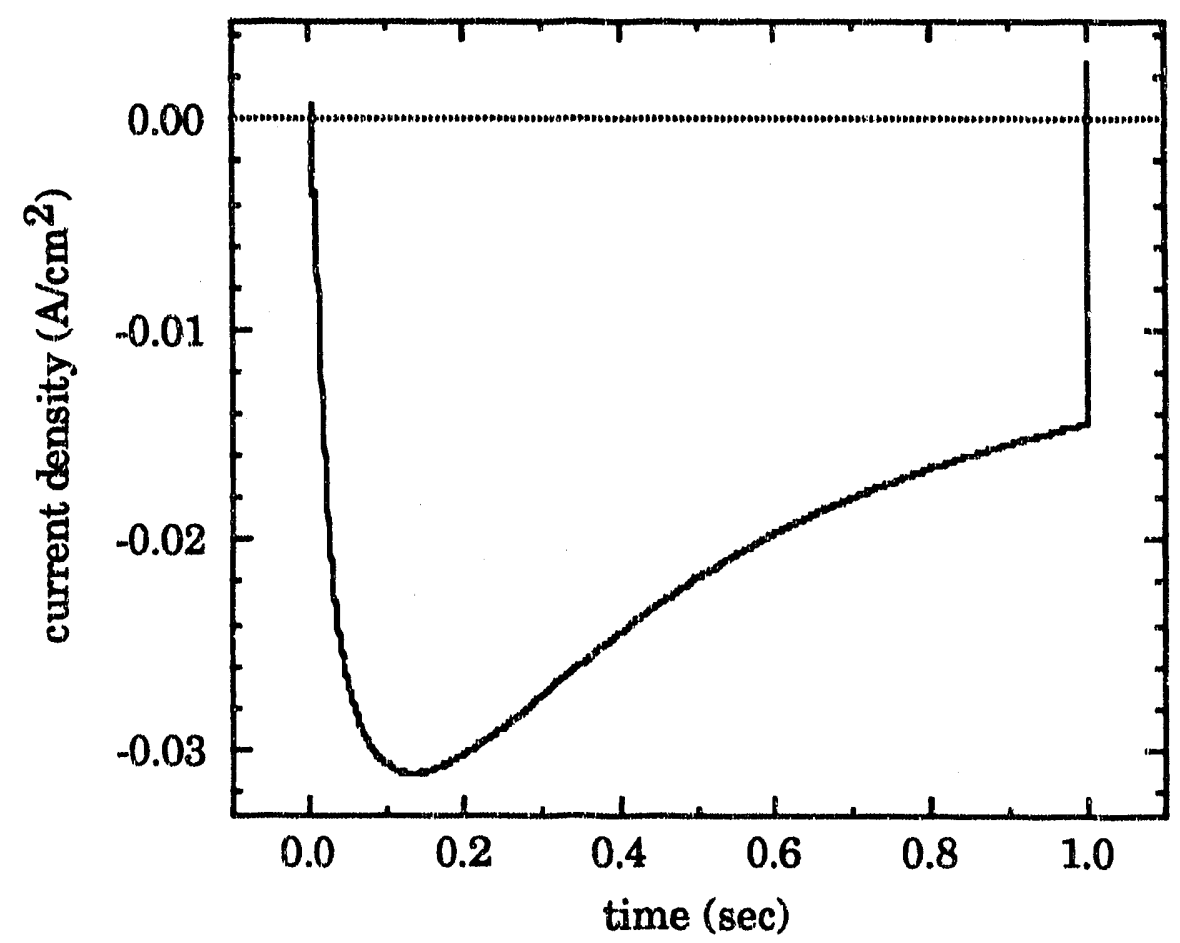

Figure 3-6: Current transient from potential step to $-700 \mathrm{mV}$ for 1 sec. in $50 \mathrm{mM}$ $\mathrm{CuSO}_{4}$ and $1 \mathrm{M} \mathrm{H}_{2} \mathrm{SO}_{4}$ electrolyte. Copper is deposited onto an HOPG electrode with the potential measured versus a copper reference electrode.

The electrochemical activity of graphite is known to increase with disorder. ${ }^{1}$ The disorder can be induced by electrolytic activity, laser heating, mechanical polishing or chemical treatment. ${ }^{2}$ In this study, however, we are trying to deposit thin layers of copper on graphite, without altering ihe surface conditions. We will try to avoid these very high applied potentials. Since we are using graphite as a model substrate and wish to extrapolate the findings from this study to other low electron density substrates, we have not employed techniques that increase the surface activity that can not be readily applied to other substrates. 


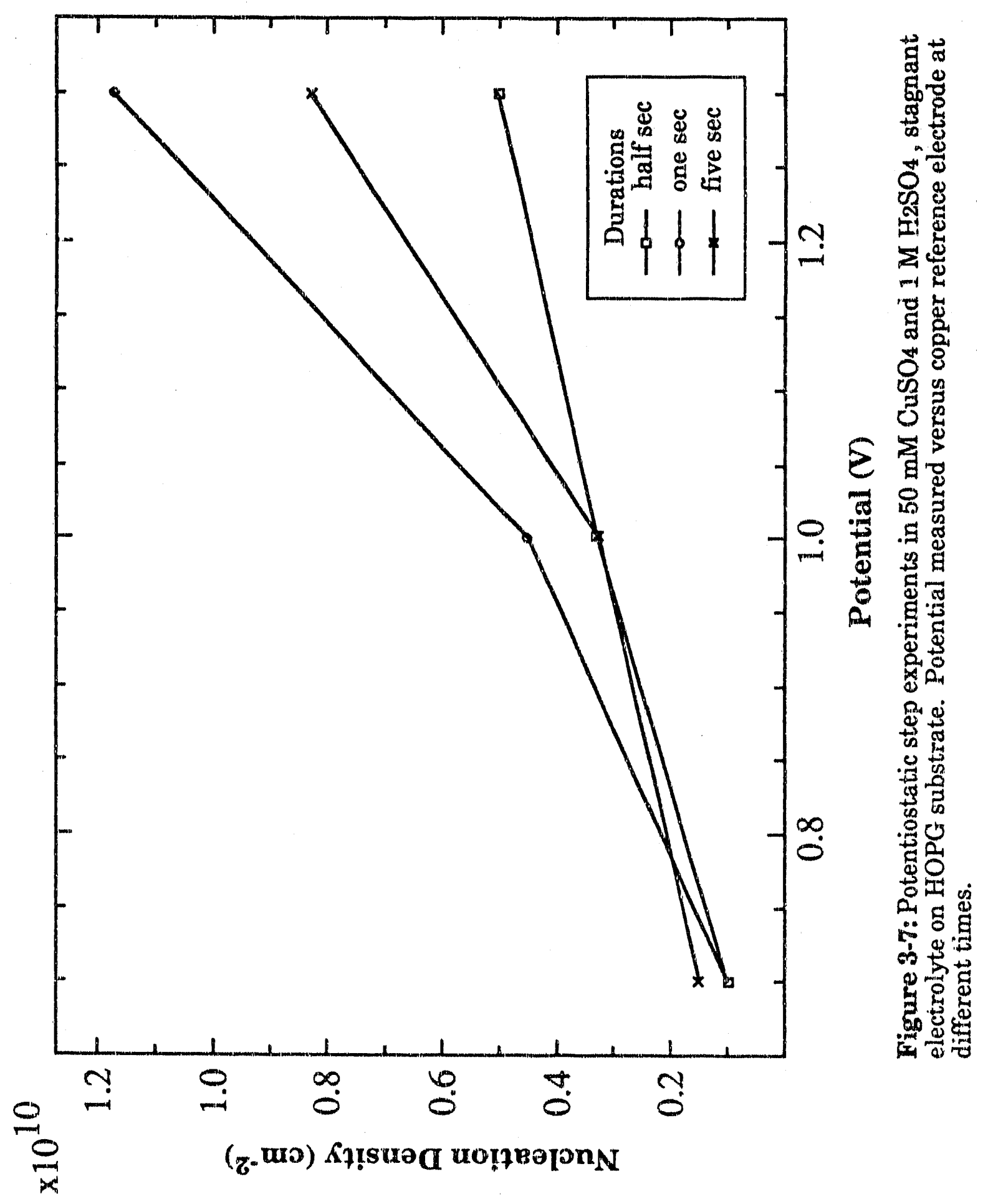




\section{Effect of Duration:}

The effect of the length of the potential pulse was examined to determine whether instantaneous or progressive nucleation occurs. With instantaneous nucleation the number density should remain the same, independent of the depasition duration. With progressive nucleation the number density of nodules should increase with duration of the potential step.

Figure 3-8 is a plot of the nucleation density versus the deposition duration. These experiments were potential steps in $50 \mathrm{mM} \mathrm{CuSO}_{4}$ and $1 \mathrm{M} \mathrm{H}_{2} \mathrm{SO}_{4}$ for various lengths of time. The potential is measured with respect to the copper reference electrode. The nucleation density remains relatively constant with duration, except for the experiment at $-1.3 \mathrm{~V}$, which has a rise followed by a decrease in the measured. nucleation density.

The decrease in the average nucleation density may indicate the redistribution of copper. However, the scatter in the data makes absolute determination impossible. Further studies revealed that redistribution of material is a problem that must be kept in mind when doing experiments that involve materials of widely differing surface energies. (See section 2.2)

The SEM images of the $-700 \mathrm{mV}$ series for durations $0.5,1.0,2.0$ and 5.0 seconds is presented in figure 3-9. Though the sizes of the nodules increase with time, their number density remains relatively constant. Changes in the nucleation density, as it depends on duration, are insignificant in comparison to the uncertainty in measurements. 


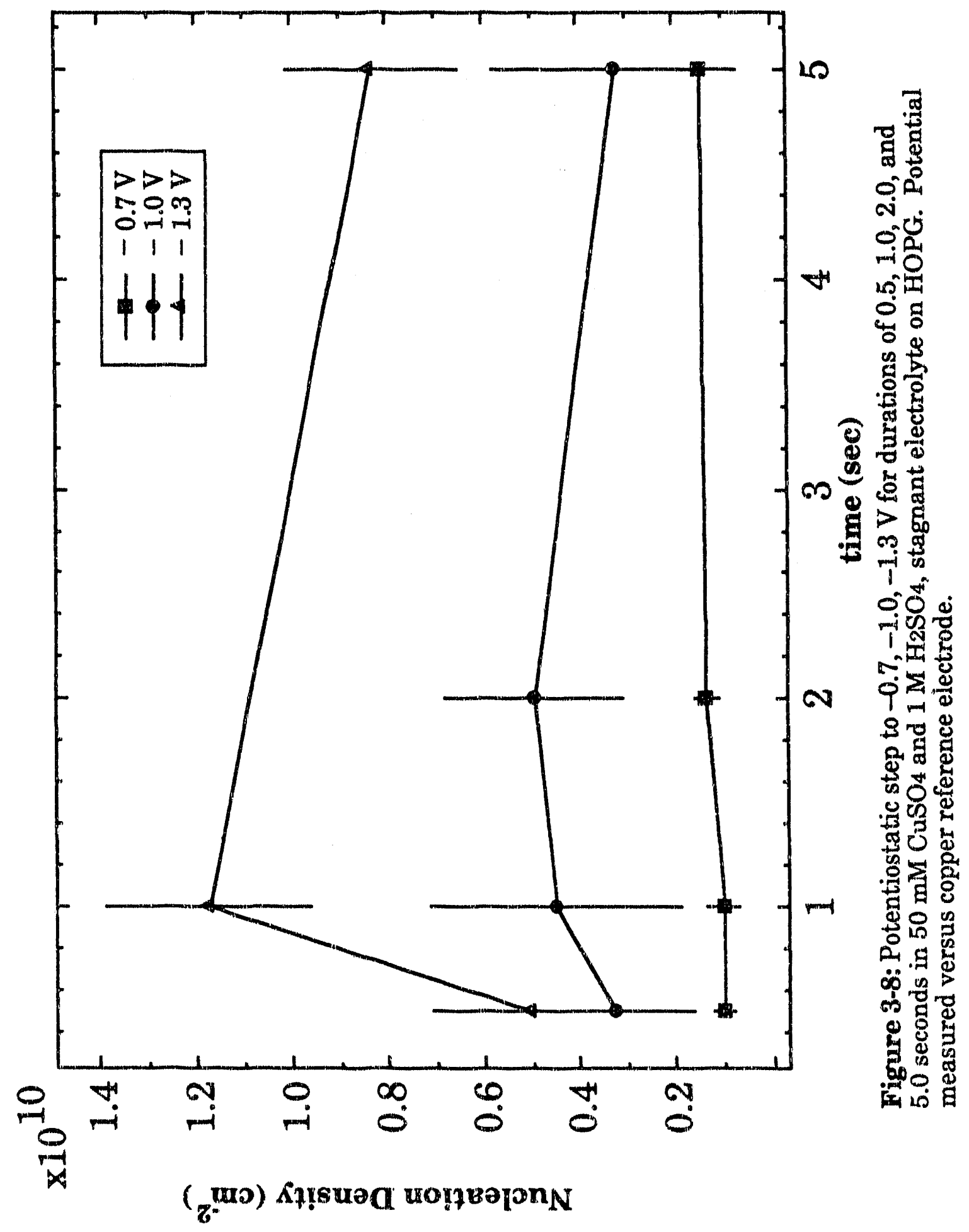



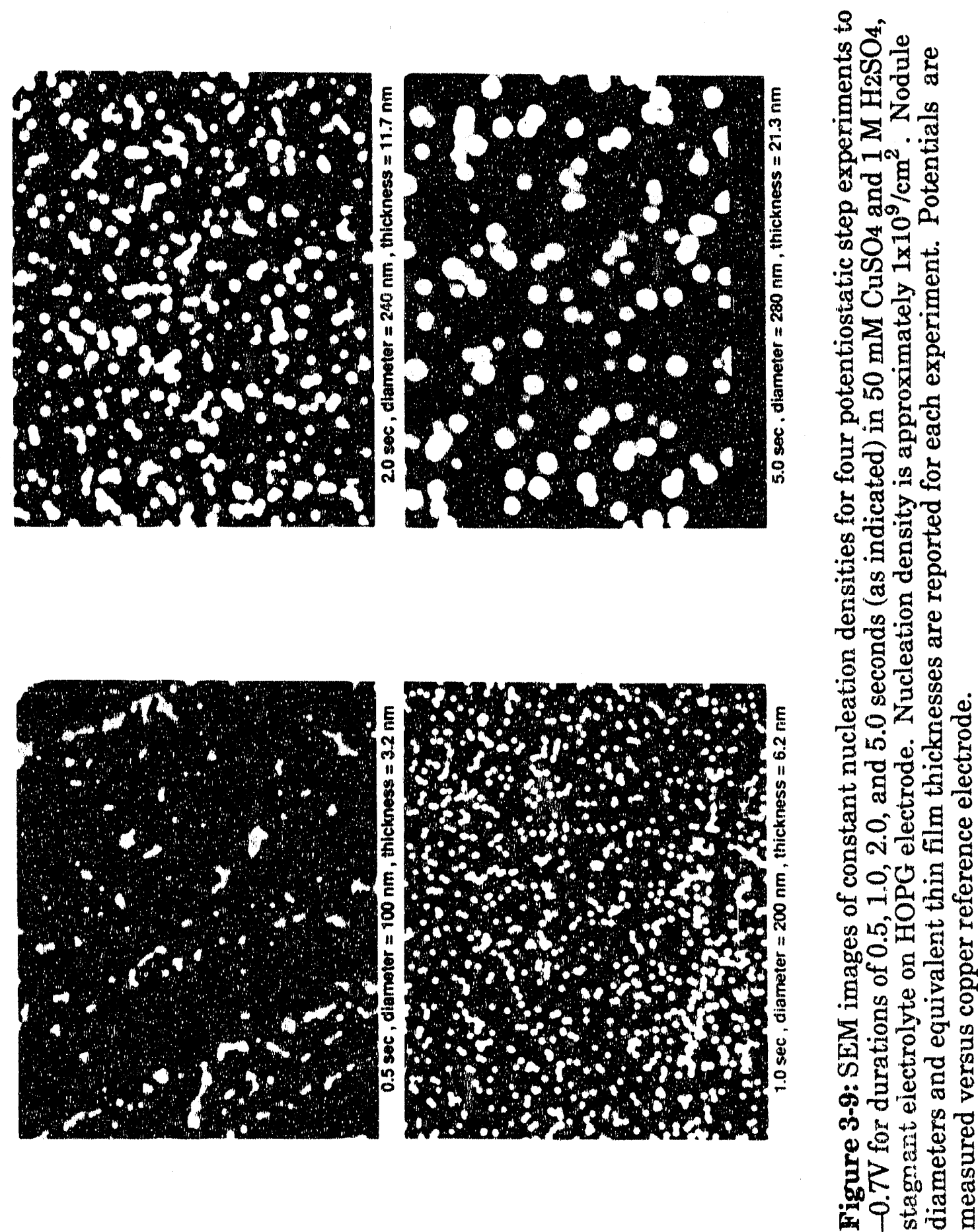


\section{Effect of Concentration:}

A variable, expected to affect the nucleation density strongly, is the concentration of copper ions in the electrolyte. Yet simple potential step experiments could not be performed with low concentrations of copper. Hydrogen reduction becomes an increasing problem at lower copper concentrations and the nodules that do form are too small and therefore unstable. The following procedure was used to make possible the study of electrodeposits from low copper ion concentration electrolytes.

In these experiments, instead of applying a constant potential, a relatively high potential was applied for a short time, then it was dropped to where hydrogen does not reduce but copper deposition continues. The potential is held at this low value until the nodules are large enough to be stable, and visible in the SEM, then the surface is analyzed. The pulse potential for these experiments was $-1.0 \mathrm{~V}$ for 0.1 seconds and the growth potential was $-300 \mathrm{mV}$. The growth was continued until the same amount of total charge was passed in each experiment. These experiments showed no dependence on concentration between $5 \times 10^{-6}$ and $5 \times 10^{2} \mathrm{M}$.(figure 3-10) Only at $5 \mu \mathrm{M}$ copper ion concentration, a significant drop in nucleation density is detected.

We conclude from these experiments that the nucleation process is not mass transport limited, unlike the growth process. The number density of nuclei is most likely controlled by the kinetics of copper nucleation on graphite. 


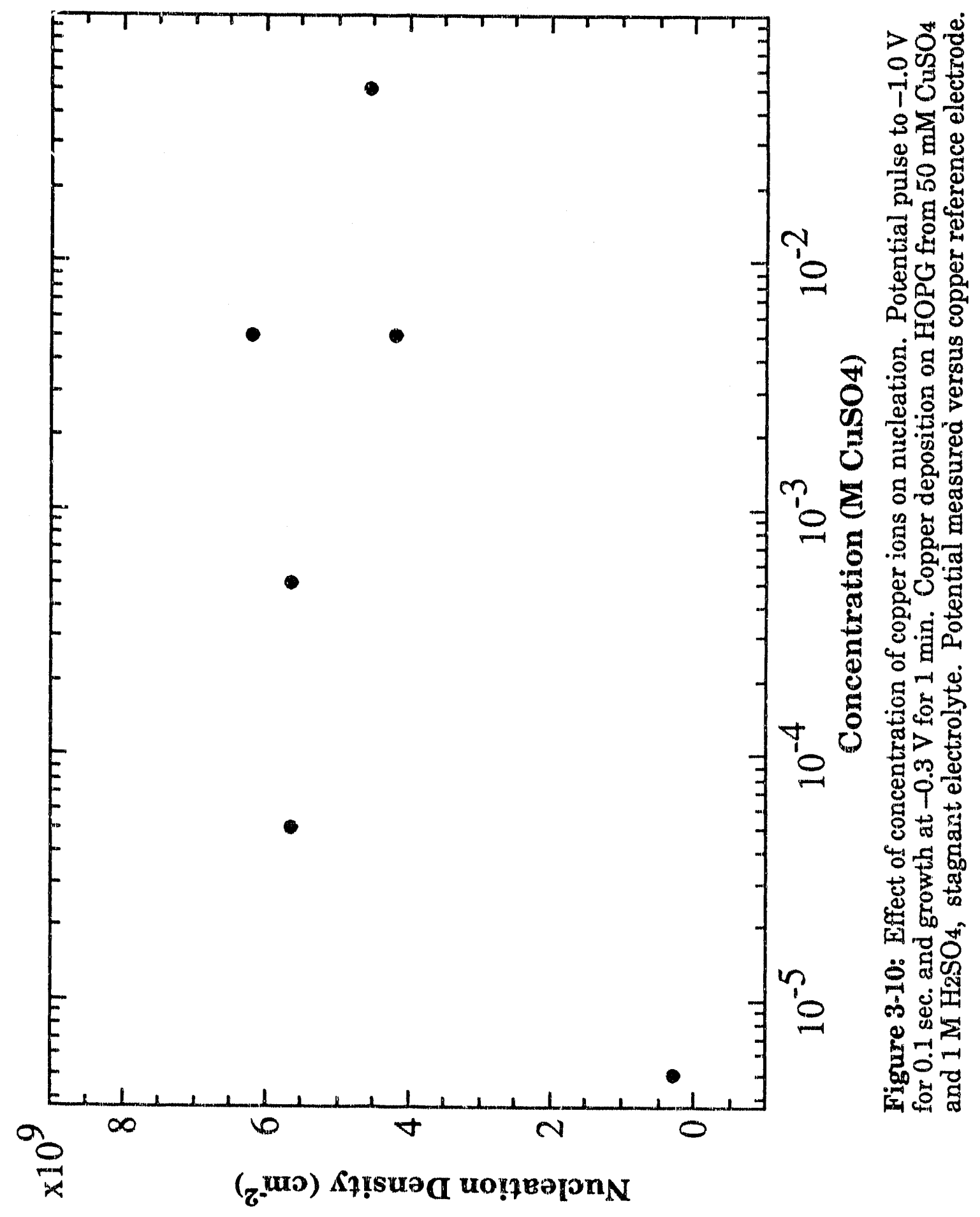




\section{Effect of Repeated Pulse Experiments:}

To take advantage of the powerful effect potential has on the nucleation density, yet avoiding complications arising from hydrogen evolution, a pulsed potential profile as illustrated in figure 3-11, was applied.

The potential is pulsed to a high value for a short time, $100 \mathrm{msec}$ in most experiments, which is long enough to seed the surface with copper nuclei, yet not long enough to cause much hydrogen evolution. The potential is then dropped and held at a lower value, where copper nuclei are grown, without any hydrogen reduction. During this one minute period, the nuclei grow large enough to be stable. The potential is then dropped to nearly open circuit. At this low potential the copper growth stops and the concentration field relaxes, in preparation for the next pulse. The potential is held slightly cathodic of open circuit to minimize copper redistribution. The procedure is then repeated several times.

These experiments decouple the nucleation reaction from the growth reaction and take advantage of the high potential pulses while eliminating unwanted side reactions. The amount of charge that is lost to hydrogen evolution is an insignificant portion of the total charge passed, because the pulse, where hydrogen can evolve, is short, and most of the charge is passed during the growth step, where no hydrogen evolution occurs.

These repeated pulse experiments lead to very high nucleation densities, on the order of $10^{10}$ nuclei per $\mathrm{cm}^{2}$. The nucleation density increases with each consecutive pulse. An SEM image of one such surface is shown in figure 3-12 along with results from earlier experiments for comparison. The first photo is an SEM image of a galvanostatic experiment. The potentials during this experiment were between -660 and $-450 \mathrm{mV}$, which resulted in a nucleation density of $2 \times 10^{8} / \mathrm{cm}^{2}$. The second photo shows that the nucleation densily is increased $\left(1 \times 10^{9} / \mathrm{cm}^{2}\right)$ when a 


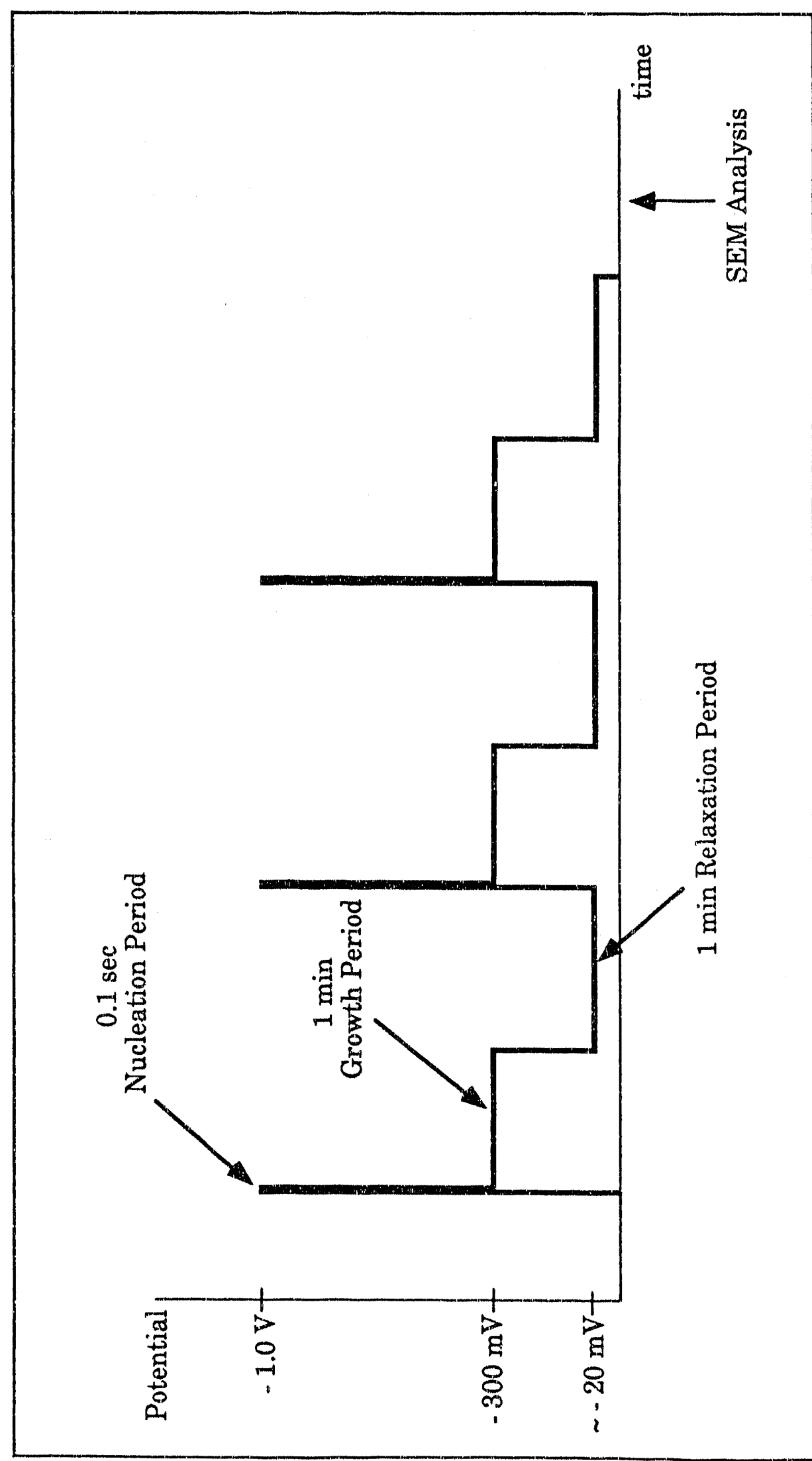

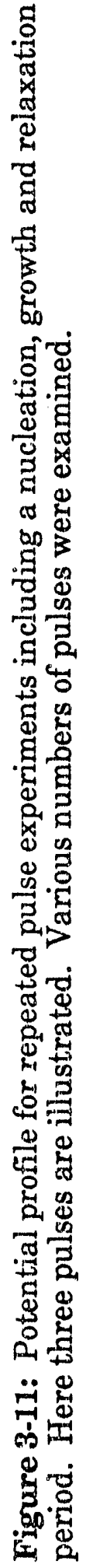


higher potential is applied. In this case, the potential was $-700 \mathrm{mV}$ for 1 second. The last photo in the series is the result from a repeated pulse experiment. The repeated pulses were applied five times with the following potentials: $-1 \mathrm{~V}$ nucleation pulse for 0.1 seconds, $-300 \mathrm{mV}$ growth potential for 1 minute, $-50 \mathrm{mV}$ relaxation potential for 1 minute. The nucleation density is increased yet another order of magnitude to $10^{10}$ nuclei per $\mathrm{cm}^{2}$.

Results from a similar experiment analyzed using the Scanning Tunneling Microscope are included in the STM section. (see Section 2.4, figure 2-16)

\subsection{Inhibitors:}

\section{Introduction to inhibitors:}

One of the possible ways to control electrodeposition is by using chemical additives in the electrolyte, such as inhibitors. There are different proposed mechanisms by which inhibitors can alter the deposition behavior. Scme of these mechanisms involve the incorporation of the additive.

Inhibitors can raise the surface overpotential for the deposition by affecting either the kinetics of the nucleation reaction or the kinetics of the growth reaction. They alter the kinetics of the nucleation reaction by adsorbing onto the substrate in the most favored sites and excluding these sites to electrodeposition. The deposit is forced to nucleate on less favored sites, which may be more abundant, thereby increasing the number density of nuclei. Inhibitors can change the kinetics of the growth reaction by adsorbing onto the electrodeposited material and thereby slowing down the growth of the nodules relative to the nucleation reaction. ${ }^{3}$ 

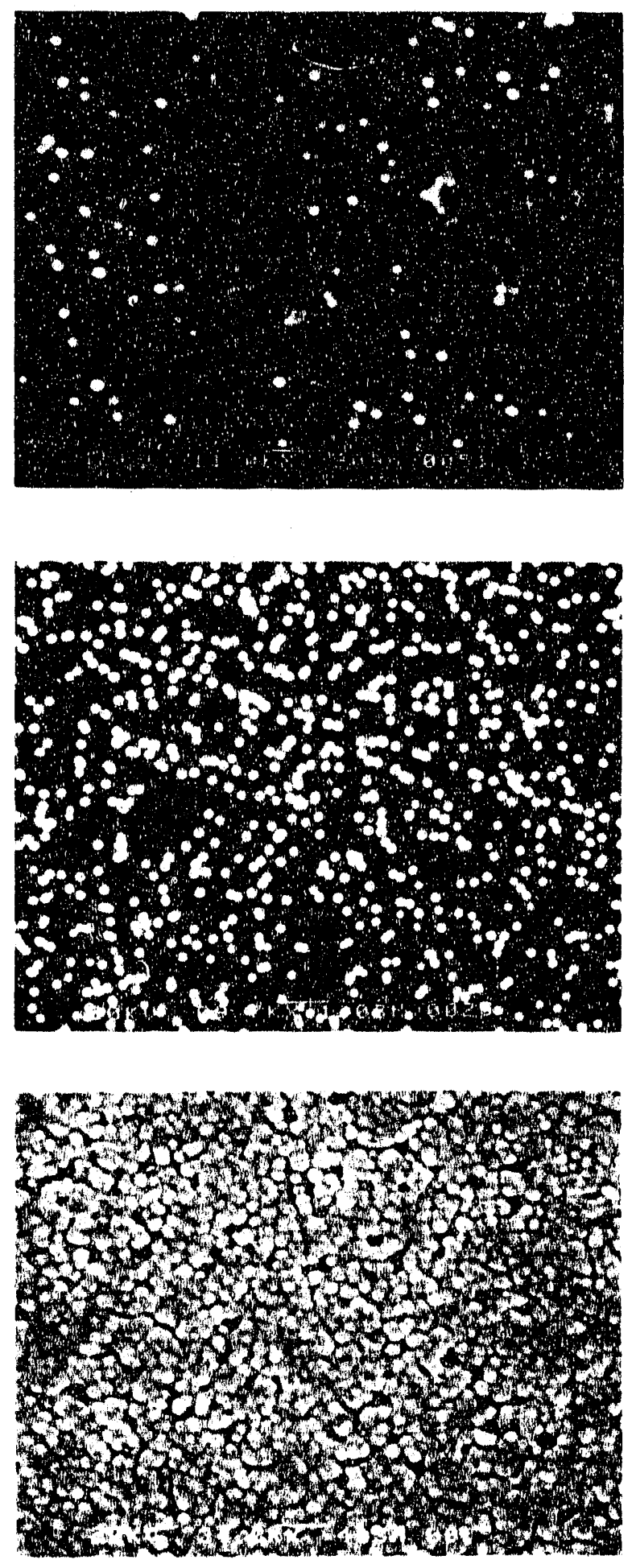

\section{Galvanostatic Pulse}

$50 \mathrm{mM}$ CuSO4 in $1.0 \mathrm{M} \mathrm{H} \mathrm{HSO}_{4}$

Current Density $=5.10 \mathrm{~mA} \mathrm{~cm}^{2}$

Duration $=2 \mathrm{~s} \theta \mathrm{c}$

Potential Range $=-660$ to $-450 \mathrm{mV}$

Nodule Density $=2 \times 10^{8} / \mathrm{cm}^{2}$

$\longmapsto 1 \mu \mathrm{m}$

\section{Potentlostatic Pulse}

$50 \mathrm{mM}$ CuSO4 in $1.0 \mathrm{M} \mathrm{H} \mathrm{HSO}_{4}$

Step Potential $=-700 \mathrm{mV}$

Duration $=1 \mathrm{sec}$

Nodule Density $=1 \times 10^{9} / \mathrm{cm}^{2}$

$\longmapsto 1 \mu \mathrm{m}$

\author{
Repeated Potential Pulses \\ $5 \mathrm{mM}$ CuSO 4 in $1.0 \mathrm{M} \mathrm{H}_{2} \mathrm{SO}_{4}$ \\ Pulse : $-1.0 \mathrm{~V}$ for $0.1 \mathrm{sec}$ \\ Growth : $-300 \mathrm{mV}$ for $1 \mathrm{~min}$ \\ Relaxation : $-50 \mathrm{mV}$ for $1 \mathrm{~min}$ \\ Repetition : 5 times \\ Nodule Density $=1 \times 10^{10} / \mathrm{cm}^{2}$
}

Figure 3-12: SEM comparison of nucleation densities on three different experiments: a galvanostatic pulse, a potentiostatic pulse and a repeated pulse experiment, with nucleation densities varying from $10^{8}$ to $10^{10}$. Experimental conditions, nucleation densities and scale bars are indicated next to each figure. 
Inhibitors can also work by complexing with the reactant ions in solution thereby changing the mass transport properties or discharge kinetics of the latter. They can also lower the surface energy of smaller nuclei by adsorbing on them and thereby stabilizing them at smaller sizes and on less favored sites. Redistribution of material from smaller nodules to larger ones is hindered. ${ }^{3}$

The two inhibitors examined in this study are Benzotriazole (BTA) and Polyvinyl alcohol (PVA). Their chemical formulae are shown in figure 3-13. Benzotriazole is a well known inhibitor to copper electrodeposition reactions. It is commonly used as both a brightening agent and a corrosion inhibitor. It is known to form a Cu-BTA film on the copper electrodeposit4, which along with adsorbed BTA is believed to be the cause of the corrosion inhibition. 5,6

\section{Conclusions from Armstrong's work:}

Armstrong ${ }^{3}$ studied the effect of BTA on copper electrodeposits on platinum substrates. He reports a $400 \mathrm{mV}$ overpotential to copper deposition under galvanostatic deposition conditions. This overpotential due to the inhibitor addition was determined during a $4 \mathrm{~mA} / \mathrm{cm}^{2}$ galvanostatic step from $0.13 \mathrm{M} \mathrm{Cu}\left(\mathrm{ClO}_{4}\right)_{2}$ and $0.1 \mathrm{M} \mathrm{HClO}_{4}$ electrolyte with $10 \mu \mathrm{M} \mathrm{BTA}$ in stagnant solution. The substrate was polycrystalline platinum. Under the same conditions, the overpotential due to PVA was measured at $870 \mathrm{mV}$. The addition of either inhibitors resulted in bright deposits.

By analyzing the nitrogen content with Secondary Ion Mass Spectrometry (SIMS) and X-ray photoelectron Spectroscopy (XPS) while depth profiling, Armstrong was able to determine that BTA was not incorporated into the electrodeposit. In fact, the nitrogen concentration was below detection limits for both techniques in the bulk of the deposit. However, the nitrogen content on the 


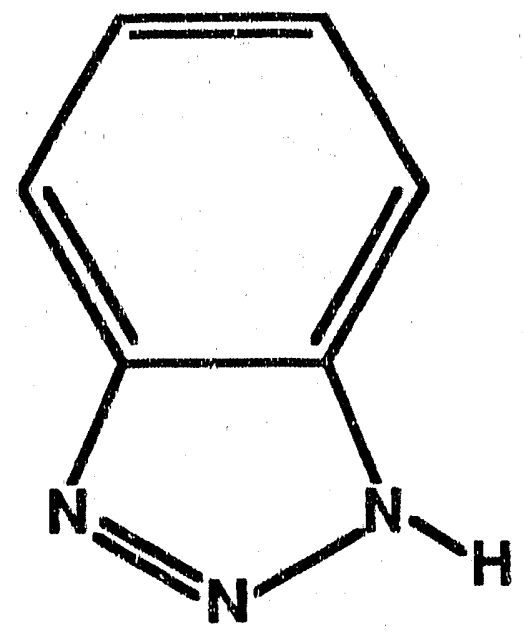

Benzotriazole $M w=119.13$

$[\mathrm{CH}-\stackrel{\mathrm{OH}}{\mathrm{C}} \mathrm{H}]_{\mathrm{n}}$

Polyvinyl alcohol

$M_{w}=70,000-100,000$

Figure 3-13: The chemical formulae and the moleculer weights of the two chemical additives studied as inhibititors to the copper electrodeposition reaction. 
surface was found to be large, showing agreement with the existence of a Cu-BTA barrier film on the surface.

His work with the scanning tunneling microscope showed changes in the growth behavior of the electrodeposit in the presence of BTA. The deposits changed from angular, crystalline shapes to rounded ones. The crystalline growth occurs because of the anisotropy between the kinetics of growth of the different crystal faces. In the presence of a Cu-BTA barrier film, however, the electrodeposit is in the typical rounded shapes indicating diffusion limited growth.

The major conclusion from his study is that BTA increases 'he nucleation density solely by raising the overpotential. The increase in the nucleation density can also be achieved without the inhibitor by directly applying the same potential that is measured in the presence of BTA in galvanostatic experiments.

\section{Effect of inhibitors on HOPG:}

The increase of overpotential on the electrodeposition of copper onto HOPG in the presence of inhibitors was determined using cyclic voltammetry (CV). Cyclic voltammetry was performed in $50 \mathrm{mM} \mathrm{CuSO}_{4}$ and $1 \mathrm{M} \mathrm{H}_{2} \mathrm{SO}_{4}$ with and without the presence of inhibitors. The inhibitor concentrations were $100 \mu \mathrm{M}$ BTA or $1 \mu \mathrm{M}$ PVA $\left(M_{W}=70,000\right.$ to $\left.100,000 \mathrm{~g} / \mathrm{mol}\right)$. All chemicals used were reagent grade. The working electrode was HOPG and the reference electrode was copper. The scan rate for all CV's was $50 \mathrm{mV} / \mathrm{sec}$ between the limits of $-1.0 \mathrm{~V}$ and $+1.0 \mathrm{~V}$. The scans were started from $0.0 \mathrm{~V}$ vers's copper, scanning initially in the cathodic direction.

\section{i. Benzotriazole:}

Figure 3-14A, the CV of copper on HOPG without any additives is identical to figure 3-2. It is reproduced here for ease in comparison with experiments were additives are present in the electrolyte (figures 3-15 and 3-16). A more detailed discussion of this CV is given in section 3.1. The most notable feature of this CV is 

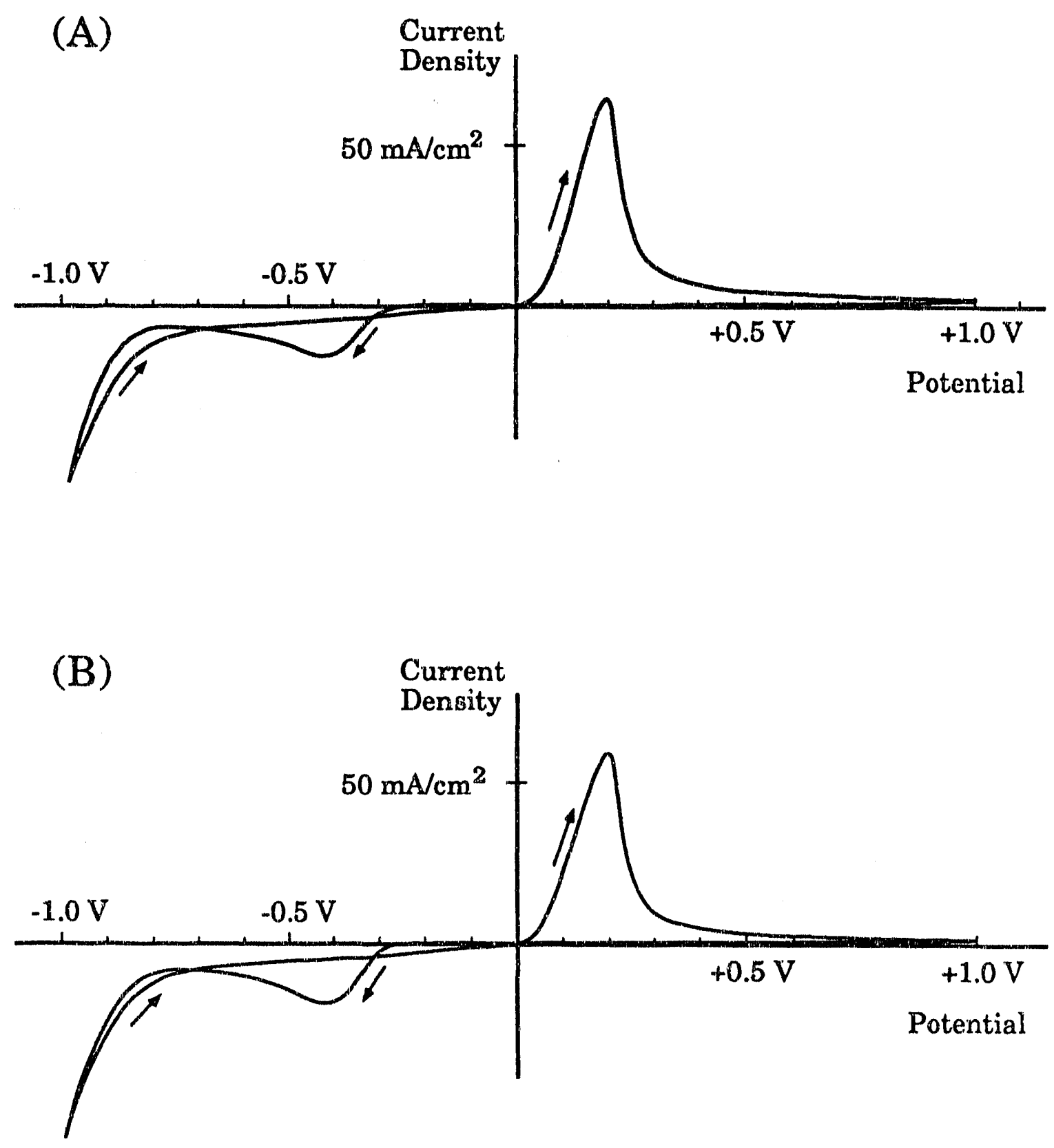

Figure 3-14: First (A) and second (B) sweeps of cyclic voltammogram on HOPG from stagnant, aqueous electrolyte containing no additives and 50 $\mathrm{mM} \mathrm{CuSO} 4$ and $1 \mathrm{M} \mathrm{H} 2 \mathrm{SO} 4.50 \mathrm{mV} / \mathrm{sec}$ scan between -1.0 and $+1.0 \mathrm{~V}$ versus copper reference electrode. 
the nucleation overpotential of copper on $\mathrm{HOPG}$ of about $-250 \mathrm{mV}$. Figure $3-14 \mathrm{~B}$ is the second scan on the same surface. It is included to show that very little change occurs on the surface even after the deposition and dissolution of copper from the surface. The nucleation overpotential remains the same.

Figure 3-15A is the cyclic voltammogram of copper on HOPG in the presence of $100 \mu \mathrm{M}$ BTA. The overpotential for copper deposition as indicated by the start of the copper reduction current is about $-300 \mathrm{mV}$. Comparing this scan with figure 3-14A, it is apparent that the overpotential is increased by about $50 \mathrm{mV}$. An added feature of this CV is the dissolution overpotential to anodic potentials, which is not seen in the absence of B'TA. This is most probably caused by the formation of $\mathrm{Cu}-\mathrm{BTA}$ complex film on the surface, which is a corrosion inhibitor. This film increases the anodic overpotential about $50 \mathrm{mV}$. The second scan, shown in figure $3-15 B$, is also very similar to the first, as in the case with no additives.

\section{ii. Polyvinyl Alcohol:}

Figure 3-16A is the first cyclic voltammogram of copper on HOPG in the presence of $1 \mu \mathrm{M}$, low molecular weight PVA $(8.5 \mathrm{~g} / 1)$. The overpotential to copper reduction is increased substantially to $-400 \mathrm{mV}$. This is a $150 \mathrm{mV}$ increase over the overpotential without PVA. The anodic sweep appears similar to the case without additives, however, visually it was observed that not all the copper is stripped off even though the current falls back to zero at high anodic potentials. This indicates an irreversible reaction between the copper electrodeposit and the polyvinyl alcohol.

The second sweep illustrated in figure 3-16B shows the effect of the remaining copper on the CV. The cathodic overpotential is strongly decreased from $-400 \mathrm{mV}$ in the first scan to $-300 \mathrm{mV}$ in the second. This decrease is evidence of copper reducing on previously deposited copper. The anodic copper dissolution peak 

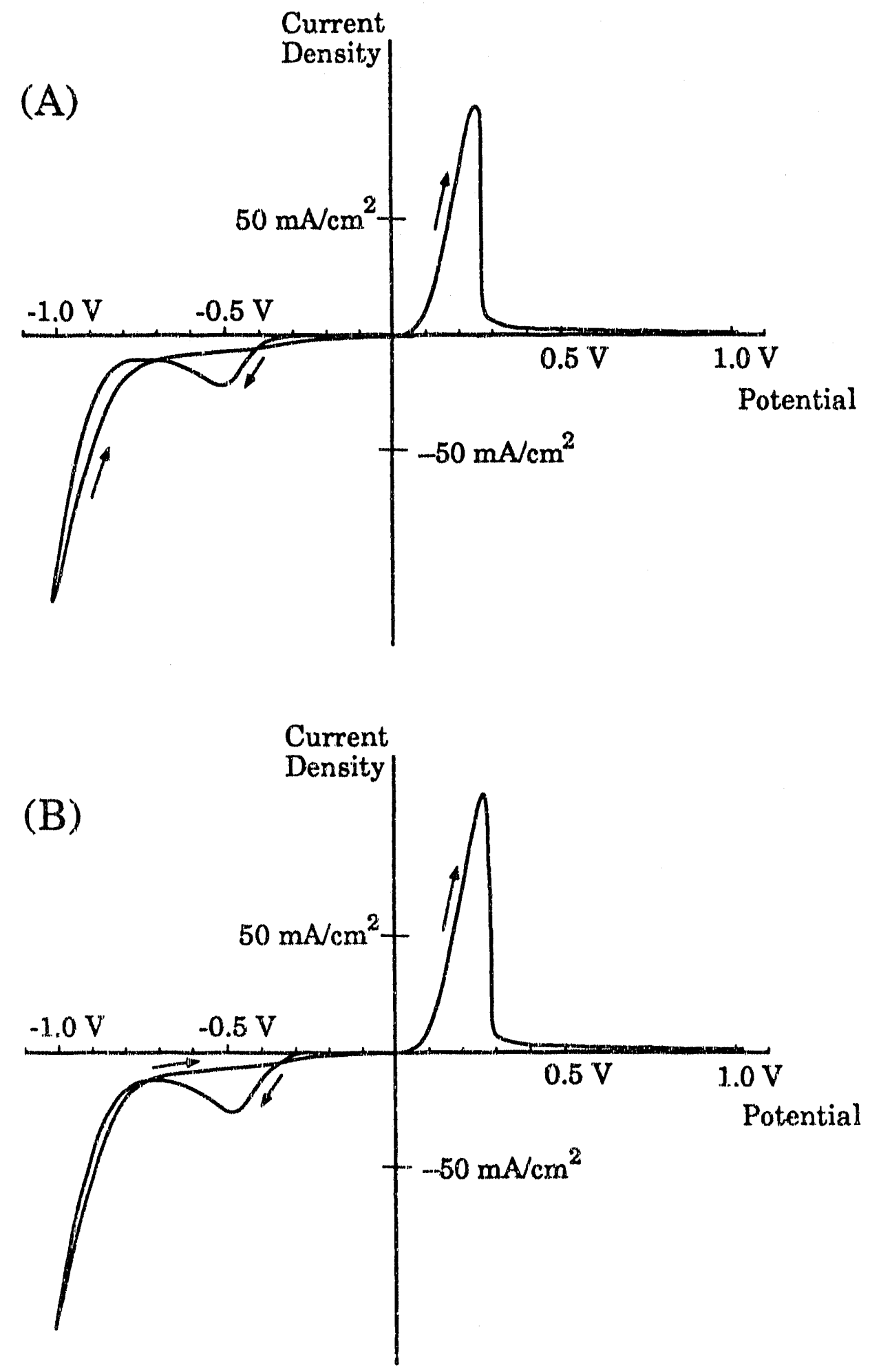

Figure 3.15: First (A) and second (B) sweeps of cyclic voltammogram on HOPG from stagnant, aqueous electrolyte containing $100 \mu \mathrm{M}$ BTA, $50 \mathrm{mM} \mathrm{CuSO}_{4}$ and $1 \mathrm{M} \mathrm{H} 2 \mathrm{SO}_{4}$. $50 \mathrm{mV} / \mathrm{sec}$ scan between -1.0 and $+1.0 \mathrm{~V}$ versus copper reference electrode. 

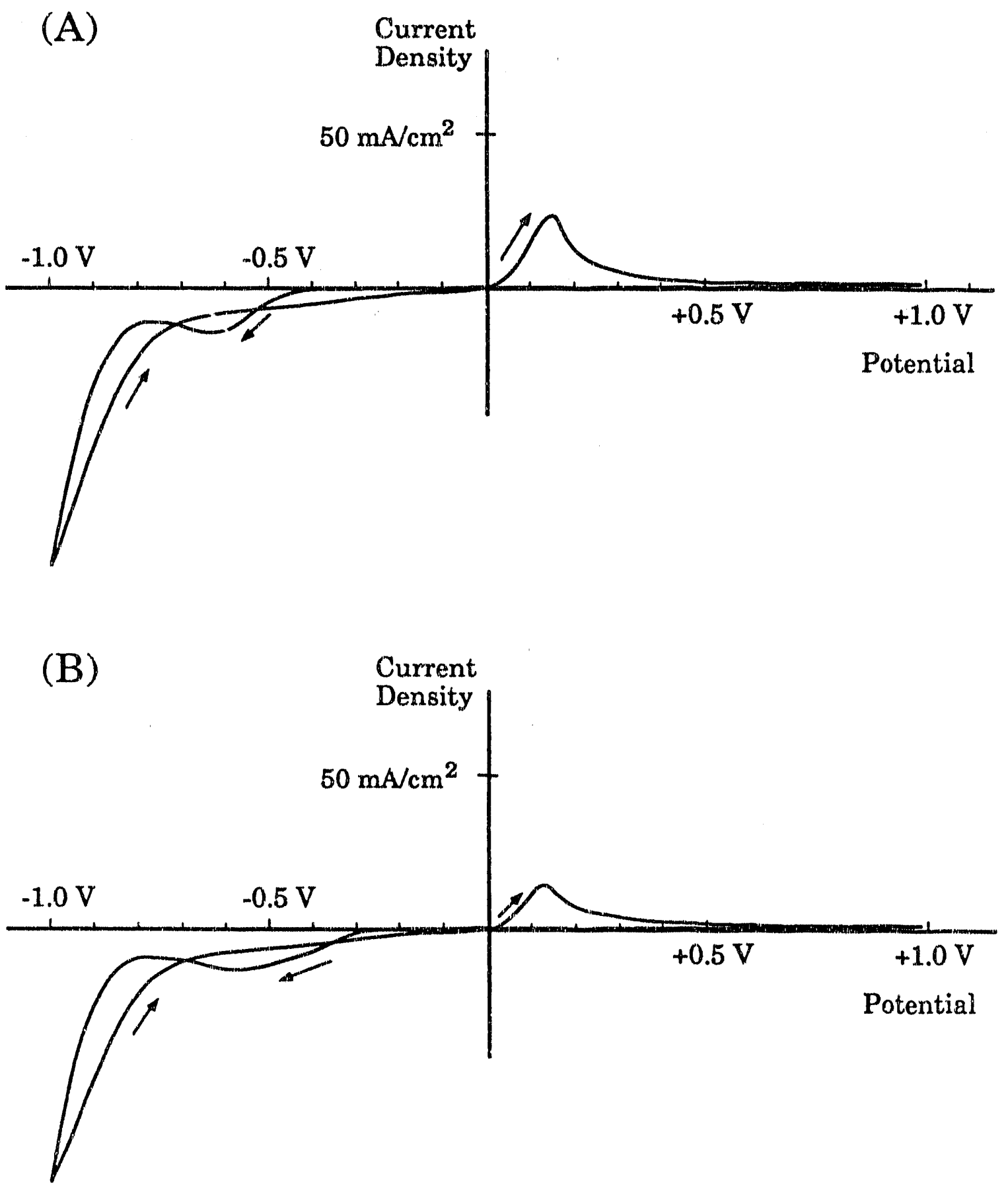

Figure 3-16: First (A) and second (B) sweeps of cyclic voltammogram on HOPG from stagnant, aqueous electrolyte containing $1 \mu \mathrm{M}$ PVA, $50 \mathrm{mM}$ $\mathrm{CuSO}_{4}$ and $1 \mathrm{M} \mathrm{H} 2 \mathrm{SO} 4.50 \mathrm{mV} / \mathrm{sec}$ scan between -1.0 and $+1.0 \mathrm{~V}$ versus copper reference electrode. 
in the second scan is smaller, as well. Even though more copper was reduced on the surface in the second scan than in the first scan, less copper is stripped off.

Visual confirmation of a copper deposit on the surface at high anodic potentials, along with the diminished anodic stripping current lead to the conclusion that PVA has formed a strong barrier to copper dissolution. Figure 3-17 is an SEM image of a HOPG surface after three potential sweeps. The sample was analyzed after returning to zero potential following the completion of the anodic sweep. There is clearly copper still on the surface. Table 3-2 summarizes the findings from the cyclic voltammetry experiments.

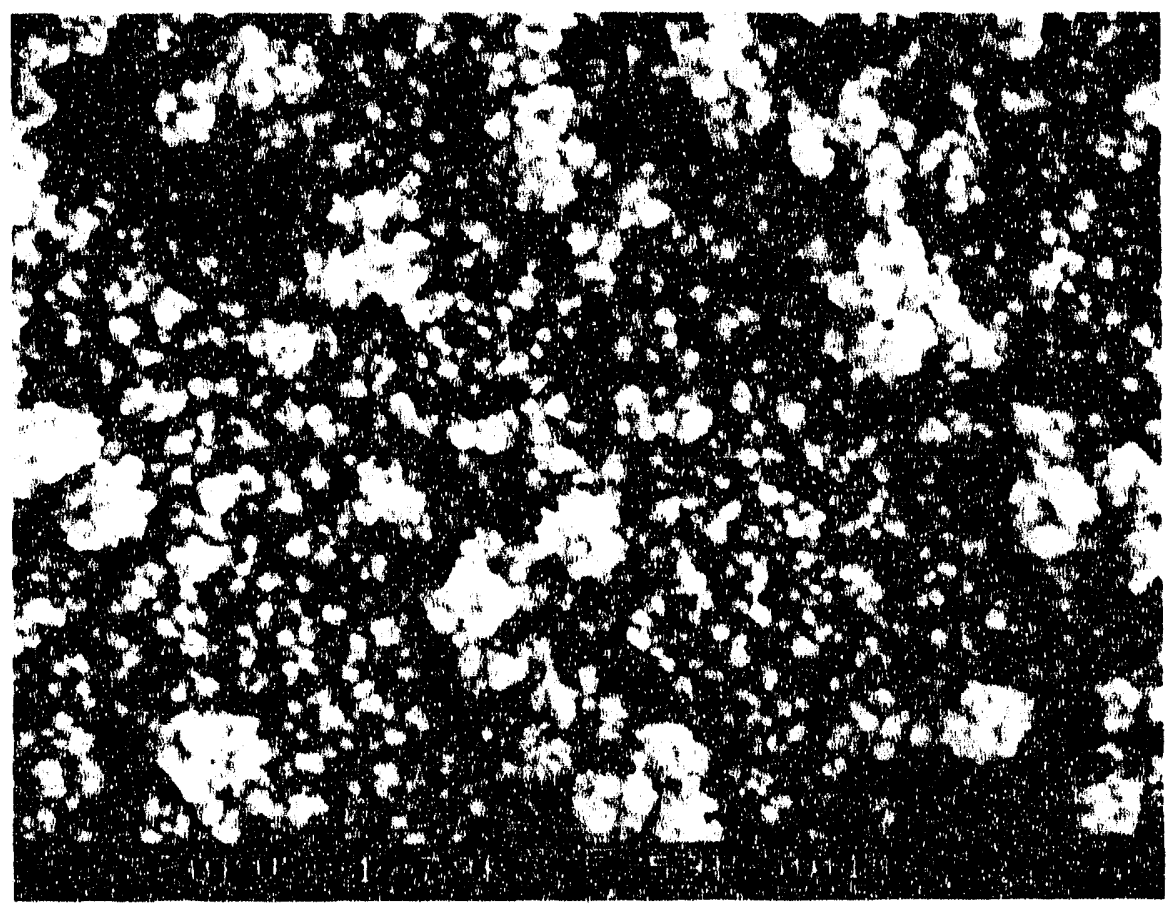

Figure 3-17: SEM of residual copper not oxidized during the anodic sweep. Deposit prepared by three potential sweeps at $50 \mathrm{mV} / \mathrm{sec}$ between -1.0 and $+1.0 \mathrm{~V}$ in $1 \mu \mathrm{M}$ PVA, $50 \mathrm{mM} \mathrm{CuSO}_{4}$ and $1 \mathrm{M} \mathrm{H}_{2} \mathrm{SO}_{4}$ on $\mathrm{HOPG}$ working electrode. Sample imaged after returning to zero potential following the completion of the anodic sweep. Potentials measured versus copper reference electrode. 


\begin{tabular}{|c|c|c|c|}
\hline Additive & $\begin{array}{c}\text { Cathodic } \\
\text { Overpotential }\end{array}$ & $\begin{array}{c}\text { Anodic } \\
\text { Overpotential }\end{array}$ & Repeated Cycles \\
\hline None & $-250 \mathrm{mV}$ & $0 \mathrm{mV}$ & same behavior as first cycle \\
\hline BTA & $-300 \mathrm{mV}$ & $50 \mathrm{mV}$ & same behavior as first cycle \\
\hline PVA & $-400 \mathrm{mV}$ & $0 \mathrm{mV}$ & $\begin{array}{c}\text { passivation of copper deposit } \\
\text { causes cathodic overpotential } \\
\text { to drop to -250 mV and the } \\
\text { anodic current to diminish }\end{array}$ \\
\hline
\end{tabular}

Table 3-2: Summary of findings from the cyclic voltammetry experiments on copper deposition on HOPG in the presence of inhibitors from $50 \mathrm{mM}$ copper sulfate and 1 $M$ sulfuric acid, aqueous electrolyte.

\section{Potential step experiments:}

Potential step experiments were performed to study the effect additives have on the nucleation density of copper on graphite. The working electrode was HOPG and the electrolyte was an aqueous solution of $50 \mathrm{mM} \mathrm{CuSO}_{4}$ and $1 \mathrm{M} \mathrm{H}_{2} \mathrm{SO}_{4}$. All experiments were performed in stagnant solution, with the HOPG electrode placed horizontally, facing up towards the copper reference electrode. The potential was stepped to $-1.0 \mathrm{~V}$ for 5 seconds. The samples were then removed from electrolyte, rinsed and dried under nitrogen, and analyzed using the scanning electron microscope. The nucleation densities reported are number density averages taken over many locations on the surface of the graphite.

The results of the potential step experiments are summarized in table 3-3. With the addition of benzotriazole, there is little change in the nucleation density. However, there is a large drop in the amount of charge that is passed. This is attributed to the BTA adsorption onto the copper surfaces, which slows down the growth of the nodules. When the growth kinetics are slow, less current passes. The nodules are smaller, yet the nucleation density does not change. This agrees well with the conclusion from Armstrong's work. BTA only increased the number density 
of nuclei by increasing the overpotential in galvanostatic experiments. The increased overpotential is responsible for the increased number of nuclei. When the potential is externally set, the number density is not affected. Because this effect is independent of the substrate used, we can conclude that BTA very selectively adsorbs onto the copper surfaces. It does not change the kinetics of the either the platinum or the graphite substrates.

With polyvinyl alcohol the results are different from those seen with BTA. The charge density is comparable to the drop in charge density with BTA, but the nucleation density is also much decreased. The kinetics of both the nucleation and the growth reactions are hindered. This seems to indicate that PVA adsorbs both onto the copper and the graphite surfaces. It is not very selective.

To test whether or not PVA adsorbs onto both graphite and copper, we repeated the experiment with PVA, yet made one small change. Instead of allowing the polymer time to adsorb onto the surface, the potential step was applied immediately as the electrolyte was placed in the cell. In the earlier experiments, the electrolyte was placed in the cell 2 to 3 minutes before the experiment was started. When this time was cut to 1 to 2 seconds, the polymer could not diffuse and adsorb rapidly enough onto the surface. The number density of nodules is much increased when the PVA is not on the surface. As shown in table 3-3 the nucleation density is very close to the numbers seen in the absence of the additive for this experiment. The charge density is also increased because of the larger number of nodules on the surface. This leads to the conclusion that the decreased nucleation density seen in the experiment where PVA was allowed to adsorb was caused by the polymer adsorbing onto the graphite. The fact that the charge density is larger than in the presence of BTA proves that PVA does not inhibit the copper growth reaction to the same extent as BTA. 


\begin{tabular}{|c|c|c|}
\hline Additive & Total Charge & Nucleation Density \\
\hline None & $148.9 \mathrm{mC} / \mathrm{cm}^{2}$ & $2.4 \times 10^{9} / \mathrm{cm}^{2}$ \\
\hline $100 \mu \mathrm{M}$ BTA & $53.2 \mathrm{mC} / \mathrm{cm}^{2}$ & $2.3 \times 10^{9} / \mathrm{cm}^{2}$ \\
\hline $1 \mu \mathrm{M}$ PVA & $50.0 \mathrm{mC} / \mathrm{cm}^{2}$ & $5.6 \times 10^{7} / \mathrm{cm}^{2}$ \\
\hline $1 \mu \mathrm{M}$ PVA* & $96.5 \mathrm{mC} / \mathrm{cm}^{2}$ & $1.0 \times 10^{9} / \mathrm{cm}^{2}$ \\
\hline
\end{tabular}

* No waiting period

Table 3-3: Results from the potential step experiments in the presence of inhibitors. All electrolytes are aqueous solutions of $50 \mathrm{mM} \mathrm{CuSO} 4$ and $1 \mathrm{M}$ sulfuric acid. Potential is stepped to $-1.0 \mathrm{~V}$ versus the copper reference electrode for 5 seconds.

Some changes in deposit shape are also evidenced when the nodules are examined at high magnification in the SEM. Figure 3-18 shows that there is renucleation of copper on the copper nodules themselves. The nodules appear as clusters of many smaller nodules. These deposits are very different form the results seen in samples prepared without PVA. Figure 3-19 is the high magnification photo of the nodules without PVA. They do not show any of the bumpy features seen with PVA. At very high magnification as in Figure 3-20 the shapes of the nodules are even easier to distinguish. This shape change gives further evidence to PVA incorporation into the deposit.

\subsection{Summary and Conclusions:}

Cyclic voltammetry was employed to quantitatively characterize the difference in electrodeposition of copper onto graphite and onto platinum. While no nucleation overpotential for copper deposition is evident on the platinum electrode, a $250 \mathrm{mV}$ nucleation overpotential is measured on the graphite electrode. The overpotential also does not occur during copper deposition on a copper covered 
graphite electrode. The nucleation overpotential is indicative of an activation barrier to copper nucleation on HOPG.

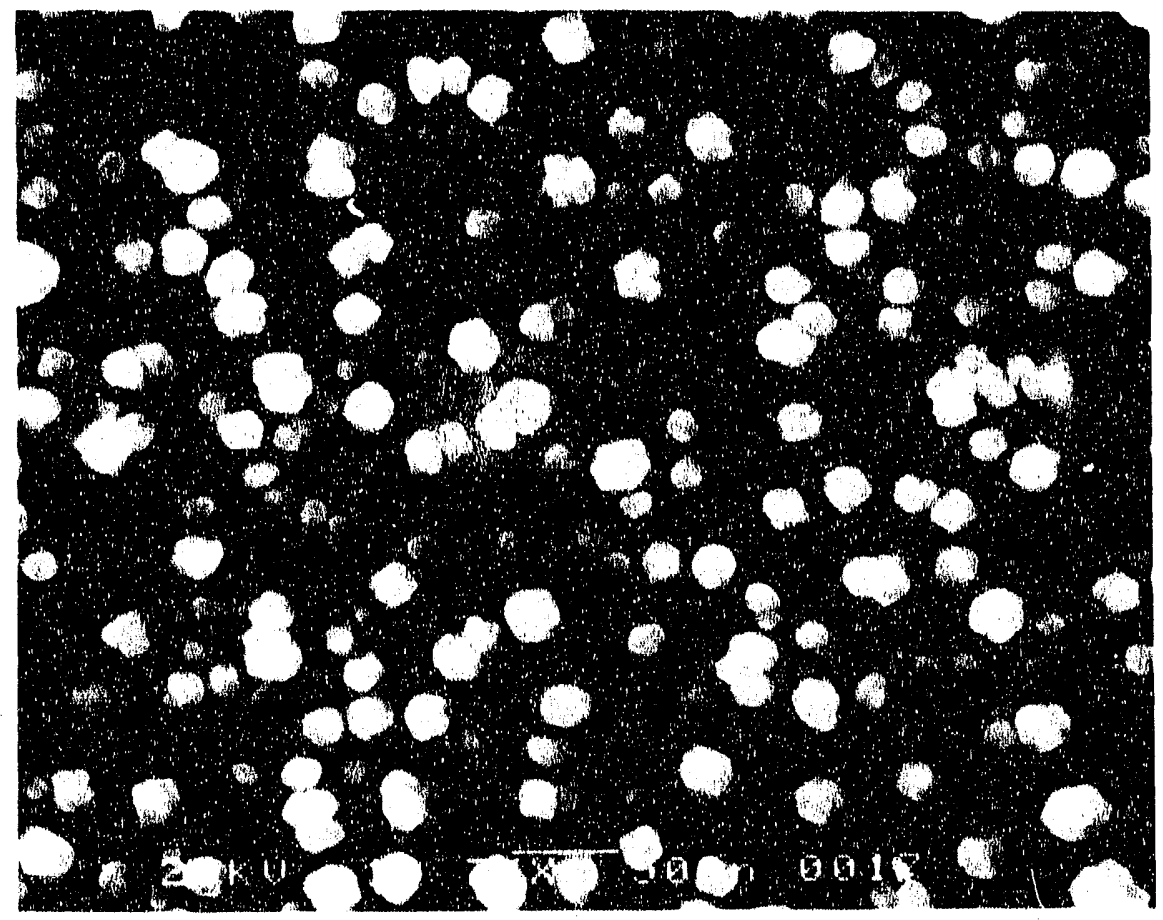

Figure 3-18: SEM of renucleated copper nodules on HOPG deposited in the presence of PVA. Copper deposited from $1 \mu \mathrm{MPVA}, 50 \mathrm{mM} \mathrm{CuSO} 4$ and $1 \mathrm{M} \mathrm{H}_{2} \mathrm{SO}_{4}$ electrolyte with a potential step to $-1.0 \mathrm{~V}$ (vs. copper) for 5 seconds.

The deposition potential is found to be the dominant parameter governing the nucleation density. There is a sharp increase in the nucleation density with applied potential. There exists a widow of potentials where copper deposition can be performed on HOPG. At low potentials, copper deposition does not occur because the potential falls below the nucleation overpotential. At high overpotentials, hydrogen evolution reduces the current efficiency.

To take advantage of the powerful effect potential has on the nucleation density, yet avoid complications arising form hydrogen evolution, a pulsed potential 
profile was applied. The surface is nucleated with a short, high potential pulse, followed by the growth of the nuclei at a lower potential, where hydrogen evolution does not occur. The growth period is followed by a period of no copper deposition and the concentration field relaxes. The procedure is then repeated with an increase in the nucleation density with each consecutive pulse. Nucleation densities on the order of $10^{10}$ nuclei/ $\mathrm{cm}^{2}$ result.

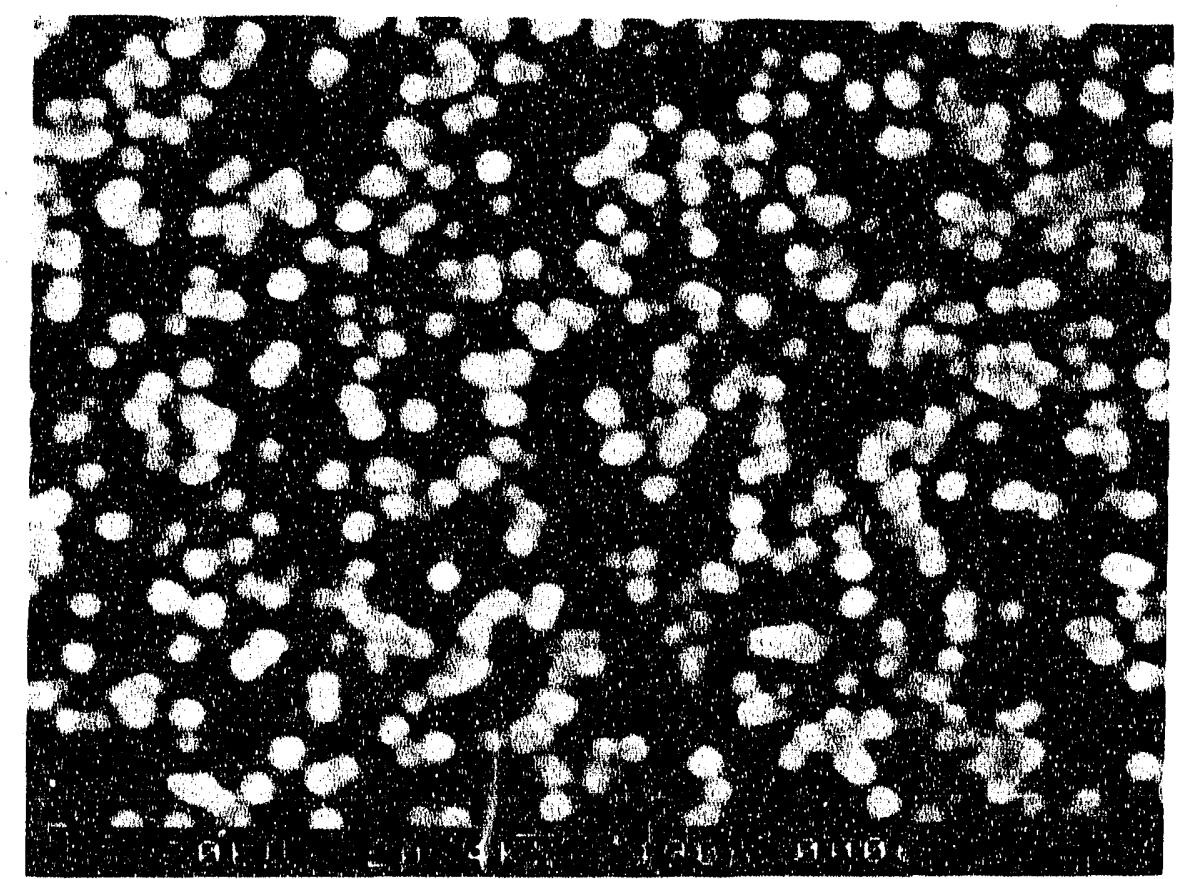

Figure 3-19: SEM of copper nodules on HOPG deposited without PVA. Copper deposited from $50 \mathrm{mM} \mathrm{CuSO}_{4}$ and $1 \mathrm{M} \mathrm{H}_{2} \mathrm{SO}_{4}$ electrolyte with a potential step to $-1.0 \mathrm{~V}$ (vs. copper) for 5 seconds.

Two inhibitors were examined to determine the effect they have on nucleation density. Polyvinyl alcohol was found to inhibit the nucleation reaction as well as the growth reaction, leading to the conclusion that it adsorbs onto both the copper and the graphite surfaces. Polyvinyl alcohol also passivates the deposited metal against anodic stripping, which indicates incorporation of the polymer into the 
electrodeposit: Under potentiostatic conditions, BTA reduces the current, but does not affect the nucleation density, leading to the conclusion that BTA adsorbs more selectively to copper than to graphite.

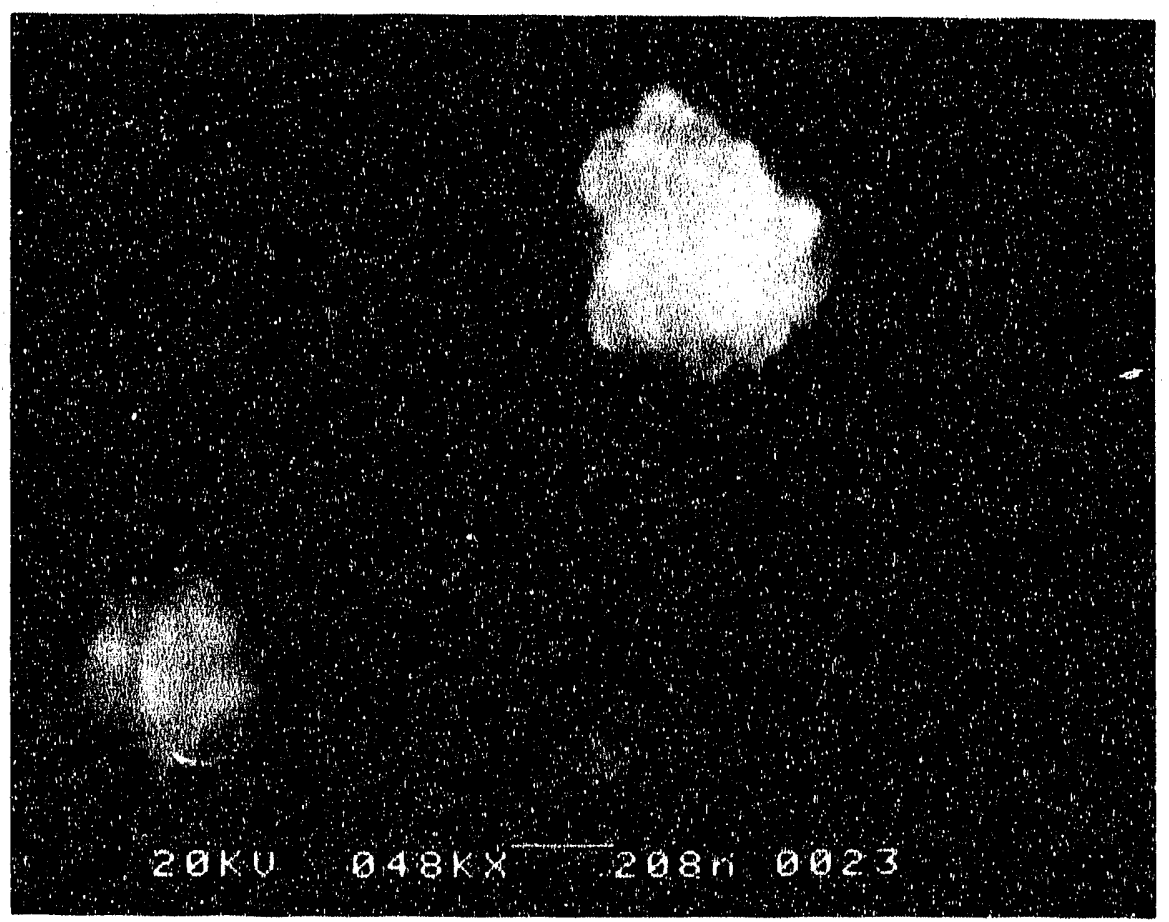

Figure 3-20: High magnification SEM of renucleated copper nodules on HOPG deposited in the presence of PVA. Copper deposited from $1 \mu \mathrm{M} \mathrm{PVA}, 50 \mathrm{mM} \mathrm{CuSO}_{4}$ and $1 \mathrm{M} \mathrm{H}_{2} \mathrm{SO}_{4}$ electrolyte with a potential step to $-1.0 \mathrm{~V}$ (vs. copper) for 5 seconds. 


\section{References:}

1 R.S. Robinson, K. Sternitzke, M.T. McDermott, R.L. McCreery, "Morphology and Electrochemical Effects of Defects on Highly Oriented Pyrolytic Graphite”, J. Electrochem. Soc. 138, 2412 (1991).

2 K. Kinoshita, in Carbon: Electrochemical and Physicochemical Properties, Wiley, New York, 1988.

3 M.J. Armstrong, "The Role of Inhibitors During Electradeposition of Thin Metallic Films", Ph.D. Thesis, University of California, Lawrence Berkeley Laboratory, LBL-28972 (1990).

4 S.L.F.A. DaCosta, J.C. Rubim, S.M.L. Agostinho, "Spectoelectrochemical Study of the Corrosion of a Copper Electrode in Deaerated $1.0 \mathrm{M} \mathrm{HCl}$ Solutions Containing Fe(III): Effect of the Corrosion Inhibitor Benzotriazolen, J. Electroanal. Chem. 220, 259 (1987).

$5 \quad$ N.D. Hobbins, R.F. Roberts, "An Ellipsometric Study of 'Thin Films Formed on Copper by Aqueous Benzotriazole and Benzimidazole", Surf. Tech. 9, 235 (1979).

6 M.M. Musiani, G. Mengoli, M. Fleischmann, R.B. Lowry, “An Electrochemical and SERS Investigation of the Influence of $\mathrm{pH}$ on the Effectiveness of Some Corrosion Inhibitors of Copper", J. Elect anal. Chem. 217, 187 (1987). 


\section{Chapter 4:}

\section{CONDUCTING POLYMER ELECTRODES}

The results from our investigations on deposition on graphite serve as a model for deposition onto other low electron density substrates such a.s conducting polymers and semiconductors. One application of metal filmis on polymer substrates is that of metal polymer multilayer structures. In particular, X-ray optical devices employ these types of structures, requiring very thin ( $\approx 10$ nanometers), continuous films with sharp interfaces. We conducted preliminary studies on the copperpolyaniline system. These materials were chosen because they both can be deposited electrolytically.

Electroactive polymers, such as polyaniline, have the property of switching reversibly between two states with widely differing conductivities. The method of conduction in these polymers is electronic due to extended $\pi$-conjugated systems. The difference in conductivity between the two states can be als much as a factor of $10^{9}$, as it is in the case of polyacetylene. ${ }^{1}$ In their conductive state, their conductivities can be $10^{3} \mathrm{~S} / \mathrm{cm} .{ }^{2}$ Switching between the two forms is accomplished by varying the oxidation state of the polymex by e/ ectrolytic uxidation or reduction. The switch is generally accompanied by a color cl.1ange.

Electroactive polymers can be produced by either standarid chemical or electrochemical synthesis. A good overview of preparation techniques for various conducting mlymers by Diaz and LaCroix ${ }^{3}$ discusses the effects ofl the solvent, the electrochemical conditions during film growth, temperature and the influence of the electrolyte anion on the electrical conductivity of various polymers. Conductive forms of electroactive polymer films are found to be a composite of the polymer in its 
oxidized form plus the anion of the electrolyte. This reference also includes recent studies, which have examined the use of polymer blends, an electroactive polymer with an inert host polymer, to improve the electrochemical and mechanical properties of these materials. A detailed discussion of proposed applications of conducting polymers is included in a review article by Diaz, Rubinson and Mark. ${ }^{4}$

\subsection{Polyaniline:}

Polyaniline (PANI) is different from other conducting polymers because it is best polymerized from aqueous solutions. ${ }^{3}$ Since the state change reaction also takes place in aqueous acid media, the use of organic solvents can be avoided. This property of PANI is an advantage when considering large-scale industrial processes.

In 1980, Diaz first prepared thin cohesive films of PANI by electrooxidation of aniline in aqueous acid solution on a platinum electrode by cycling the voltage between -0.2 and $0.8 \mathrm{~V}$ versus the saturated calomel electrode. 5 Gradually reducing the anodic potential limit to maintain current densities below $0.4 \mathrm{~mA} / \mathrm{cm}^{2}$ minimizes the formation of degradation products. ${ }^{3}$ The quality of the films depends on the potential function applied to oxidize the films. Lower quality films result from oxidizing by a potential step rather than by potential cycling. 6 The material previously had been formed by chemical synthesis, which results in a precipitate that can be pressed into pellet electrodes. ${ }^{7}$

The polyaniline switching reaction is not completely understood. The conversion to the conducting state occurs gradually as the film is oxidized. It is known to switch in the potential range between 0.1 and 0.8 Volts anodic of the hydrogen electrode. 4 The conductive state is $10^{6}$ times more conducting than the insulating state. 4 PANI is electroactive in acidic solutions $(\mathrm{pH}<3-4)$ but loses the ability to change state in neutral or basis solutions. ${ }^{8}$ Differing mechanisms for the 
switching reaction have been proposed by Mohilner ${ }^{9}$, Diaz $^{5}$, Kitani $^{10}$, Kobayashi $^{11}$, MacDiarmid ${ }^{12}$ and Geniès ${ }^{13}$. These mechanisms are summarized in the review article by Geniès et al. 8

PANI experiences electrochromic switching similar to other conducting polymers. It is observed to be yellow at $-0.2 \mathrm{~V}$ in its reduced, neutral form and blue at $+0.6 \mathrm{~V}$ in its oxidized, conducting form. Some researchers have attributed the color changes to the concentration of electrolyte anions in the PANI film. ${ }^{14}$

Different oxidation states of PANI are pictured in figure 4-1. There is a continuum of oxidation states ranging from the completely reduced leuco-emeraldine form to the completely oxidized pernigraniline form. The corresponding colors of the film for each oxidation state are also included. The amount of Faradaic charge for the oxidation reaction is measured to be $0.16-0.29$ Faradays per repeat unit, accompanied by a large capacitive charge.5,15 Anion insertion during oxidation is still a debated issue, however work by Kobayashi et al. ${ }^{16}$ and Okabayashi et al. ${ }^{17}$ with electrogravimetry shows the weight of the polymer increases linearly upon charging (oxidation) and decreases linearly upon discharging (reduction). The weight change corresponds to an insertion/expulsion process involving the anion.

The electroactivity of PANI is preserved during storage in ambient conditions in either the oxidized or neutral form. Other conducting polymers were found to be less stable to moisture, air, and halides such as chloride and fluoride salts. ${ }^{4}$ PANI has also been found to be stable during repeated switching when the anodic potential is kept below $+0.4 \mathrm{~V} .5,11$ Tamura et al. report the PANI film being stable to $10^{6}$ switches. 11 


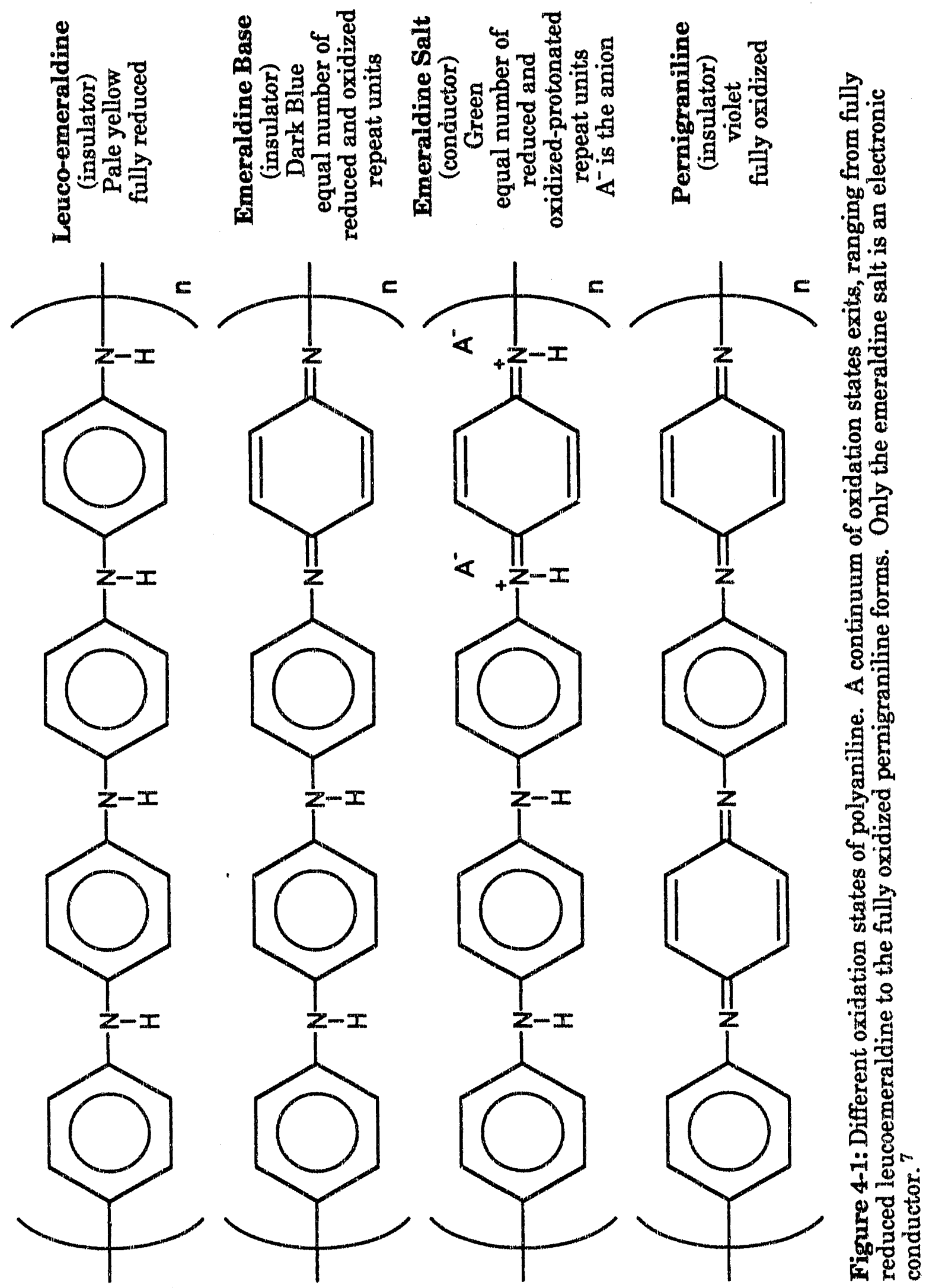


Switching raws are observed to be on the order of milliseconds. 18,19 The rate depends on the thickness of film, consistent with being limited by the anion diffusion through the polymer film.14,15 The switching time for PANI has been found to be less than that for polypyrrole, one of the principal materials reported in the literature.

Various mechanisms have been proposed for the polymerization reaction of aniline. Most researchers agree that polymerization of aniline begins with the formation of a radical cation, which is resonance stabilized. (figure 4-2) Geniès et al. describes in detail the possible propagation mechanisms to form polyaniline. ${ }^{8}$ The possible reaction mechanisms are reproduced in figure 4-3. At low overpotentials, the PANI formed results from the polymerization of the monomer radical cation. (Reaction 3) At high overpotentials, the aniline monomer is oxidized with the loss of two electrons and one proton. The polymerization reaction then proceeds as the electrophilic addition of this oxidized form of the aniline monomer to the fully oxidized polymer chain. (Reaction 5).20

The electrodeposition of conducting polymers onto metal substrates, the electrodeposition of metals onto conducting polymers, and the codeposition of the polymer with the metal have been investigated. Schirmeisen and Beck ${ }^{21}$ studied electrocoating various metals with polypyrrole. Their findings indicate that substrates that form insulating oxides such as platinum, gold, titanium and stainless steel were easily coated, however, copper dissolved anodically at potentials needed to form the polymer. Pletcher et al. 22 , from their studies on polypyrrole films, conclude that, based on analysis of the current transients, the deposition of the polymer resembles the nucleation and growth processes seen in metal deposition.

Chandler and Pletcher have studied electrodeposition of assorted metals onto polypyrrole films. ${ }^{23}$ The original motivation of their research was to deposit metals 
and polymers either in multilayers or in mixtures. They found, however, that silver, copper, nickel, platinum, palladium, ruthenium and lead, when added to the pyrrole containing electrolyte, caused spontaneous, homogeneous polymerization resulting in the formation of a black precipitate.
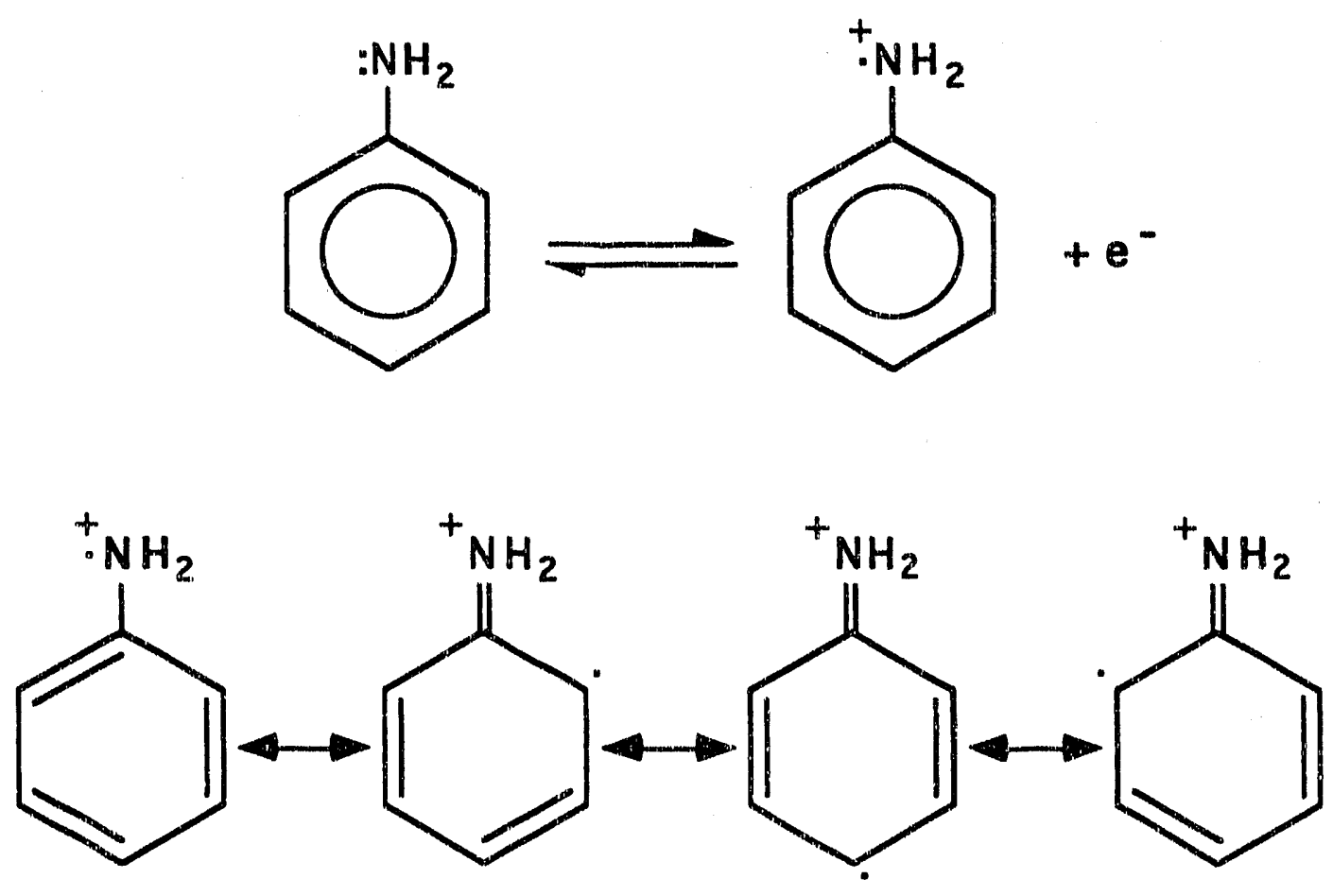

Figure 4-2: Aniline forms a resonance stabilized radical cation.

Metal deposition was performed on pre-formed polymer films, keeping the anion the same in the electrooxidation electrolyte as in the metal deposition electrolyte. The nucleation overpotential for the metal deposition reaction was observed to increase by 50 to 300 millivolts. The authors conclude that the nucleation reaction is more affected than the growth reaction by the existence of the polymer film.23 


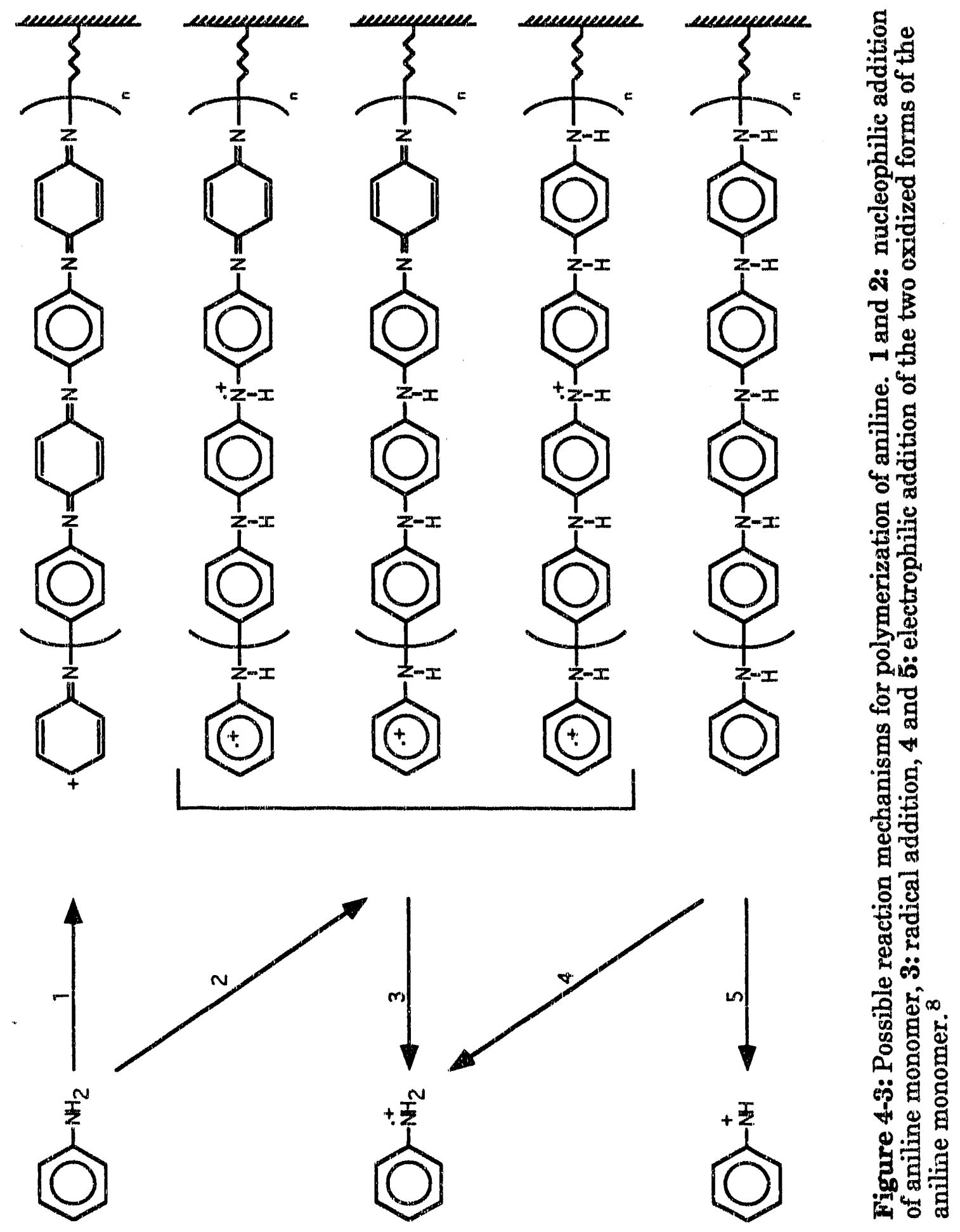


Another interesting finding was that metal deposition occurred at potentials where the polymer is expected to be insulating. This could result from deposition through pinholes in the polymer layer, such as encountered by Lee and Tan 24 in electrodeposition of copper on polypyrrole and Tsakova and Milchev ${ }^{25}$ in

electrodeposition of silver on polyaniline. However, Chandler and Pletcher ${ }^{23}$ dismiss this possibility because the same phenomenon was encountered on thick polymer films where the existence of pinholes is less likely. They also found that the current density for the reduction of ruthenium reaches the diffusion controlled value expected of diffusion to the entire geometric area of the electrode and not hemispherical diffusion to isolated metal centers in the pinholes.

Some research groups report methods of creating metal-polymer deposits other than depositing the two materials sequentially. Tian et al. ${ }^{26}$ report successfully creating small silver clusters in PANI films by first replacing the anions in the conducting polymer with metal complexes followed by reducing the entire film. Mazur et al. $27,28,29$ report producing thin metallic films in non-conducting polymers, by diffusion of metal salts and reducing agents into the polymer from opposite surfaces.

\subsection{Experimental Procedure:}

Preliminary experiments were performed to study copper deposition on polyaniline electrodes. Polyaniline was electrooxidized on a platinum substrate from sulfuric acid electrolyte. The aniline used was reagent grade, supplied by Aldrich Chemical Co. The aniline was added to $1 \mathrm{M}$ aqueous sulfuric acid in 20 to 1 volume ratio. Platinum was used for the working and the counter electrodes. The potentials for the electrooxidation were measured versus the saturated calomel electrode (SCE). 
Electrooxidation of aniline was accomplished by cycling the potential between $-0.2 \mathrm{~V}$ and $+0.7 \mathrm{~V}$ versus SCE at scan rates between 20 and $100 \mathrm{mV}$ per second. The cyclic voltammogram for the first three cycles is shown in figure 4-4. The color changes on the electrode between yellow (reduced) and dark blue/black (oxidized) were observed while cycling the potential. The polymer covered surface was removed from the electrolyte in the oxidized form and rinsed with distilled water. The polymer film was smooth, bright, and cohesive. It was inaged using both the scanning electron and scanning tunneling microscopes. (SEM, STM)

The polyaniline covered platinum electrode was then placed in $50 \mathrm{mM}$ copper sulfate containing $1 \mathrm{M}$ aqueous sulfuric acid electrolyte. Copper was deposited at $-0.1 \mathrm{~V},-0.2 \mathrm{~V}$, and $-0.4 \mathrm{~V}$ versus a copper reference electrode. The resulting surface was again analyzed using both SEM and STM.

\subsection{Results:}

Examination of the copper deposited polymer surfaces yielded images of $300 \mathrm{~nm}$ copper nodules sparsely covering a textured polymer background.

(figure 4-5) The approximate number density for the copper nocules imaged in figure 4-5 is $4 \times 10^{7} / \mathrm{cm}^{2}$. This nucleation density is comparable to the nucleation densities achieved on HOPG at low overpotentials. Very little information was found by studying the copper deposited polyaniline surfaces with the STM. Because the copper nodules were not closely packed, the nodules were difficult to discem in the images. At length scales scanned by the STM (fractions of micrometers), the likelihood of finding a nodule is small at nucleation densities as low as $10^{7} / \mathrm{cm}^{2}$. 


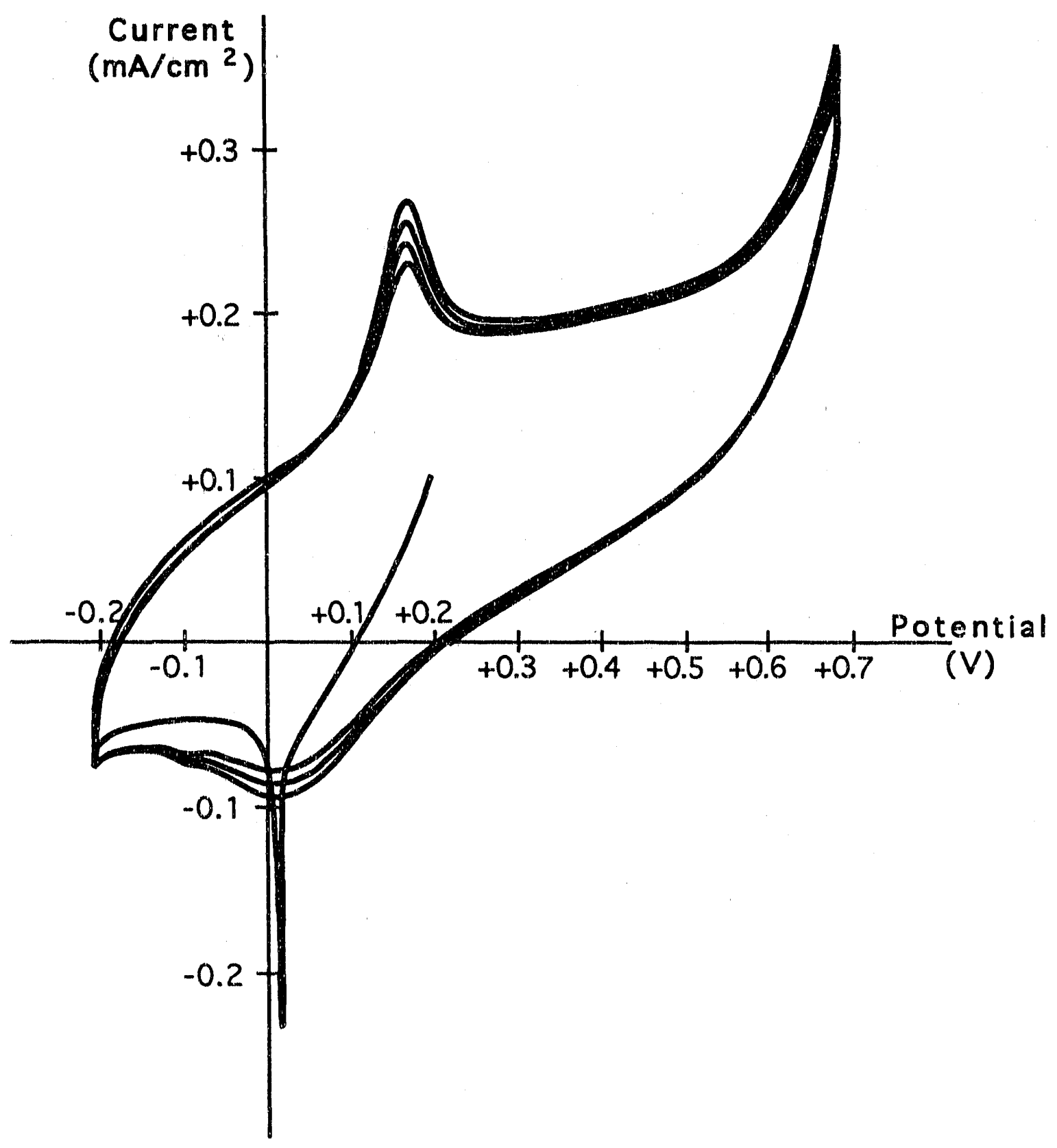

Figure 4-4: Cyclic voltammogxam during deposition of polyaniline onto platinum substrate. $20: 1$ volumetric ratio of $1 \mathrm{M} \mathrm{H}_{2} \mathrm{SO}_{4}$ and aniline. Scan rate is $50 \mathrm{mV} / \mathrm{sec}$ between -0.2 and $+0.7 \mathrm{~V}$ versus SCE. 


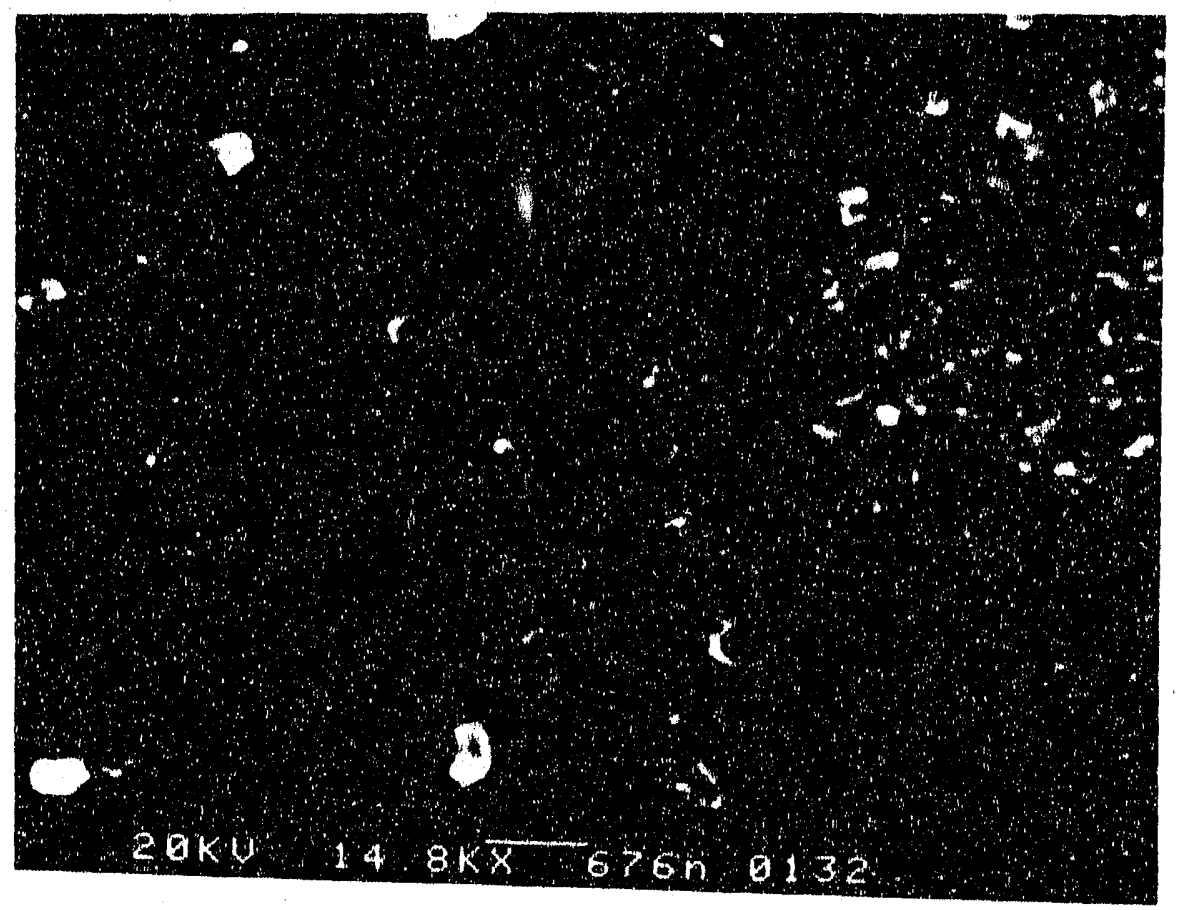

Figure 4-5: SEM of copper deposited polyaniline surface. Aniline electropolymerized by cycling between -0.2 and +0.7 volts versus SCE. Copper deposited from $1 \mathrm{M} \mathrm{H} 2 \mathrm{SO} 4$ and $50 \mathrm{mM}$ CuSO4 solution by a potential step to $-0.2 \mathrm{~V}$
versus copper reference electrode.

The standard reduction potential, $\mathrm{E}_{0}$, of copper is $+0.34 \mathrm{~V}$ anodic of the hydrogen electrode. Polyaniline switches within the range of +0.1 and $+0.8 \mathrm{~V}$ versus the hydrogen electrode. (Figure 4-6) It is in its insulating, reduced state at potentials more cathodic of this range. By examining these values we can conclude that a maximum of $0.24 \mathrm{~V}$ of overpotential can be applied for the copper deposition reaction, to keep the polymer in its conducting state while depositing the copper. However, during one of the experiments $0.40 \mathrm{~V}$ of overpotential was applied to the copper reduction reaction. Current was passed and copper deposited even though polyaniline was in its reduced, insulating state at this potential. Copper could be depositing in places where there is an imperfect covering of polyaniline, such as 
holes or cracks in the polymer surface. Thin coatings of polyaniline exhibit many pinholes, and the thick coatings develop cracks. Another possibility is that the polymer film is not completely reduced; and deposition continues on areas which are still in the conducting state.

Based on the current transient, Chandler and Pletcher 23 dismiss the possibility of depositing in pinholes. However, where there are nodule densities of $107 / \mathrm{cm}^{2}$, the hemispherical diffusion fields formed around the nodules would overlap in $\mathbf{3 0}$ seconds, resulting in planar diffusion to the geometrical area of the electrode. (Equation [2-1]) Therefore, when depositing on polymer films with a sufficiently large number of pinholes, the current transient will resemble that to a planar surface even though metal deposition is only occurring in pinholes.

Copper may not be the best metal to deposit on polyaniline. A more noble metal which has a higher $E_{0}$ can be deposited on the polymer in its conducting state with more overpotential. One such candidate, with an $\mathrm{E}_{0}$ of $+0.76 \mathrm{~V}$ versus hydrogen, is rhodium (Figure 4-6), which can be deposited from an aqueous sulfuric acid bath. Keeping the electrolyte the same between the polymerization and metal on polymer deposition steps is important for considerations involving the anion transport through the polymer film. 


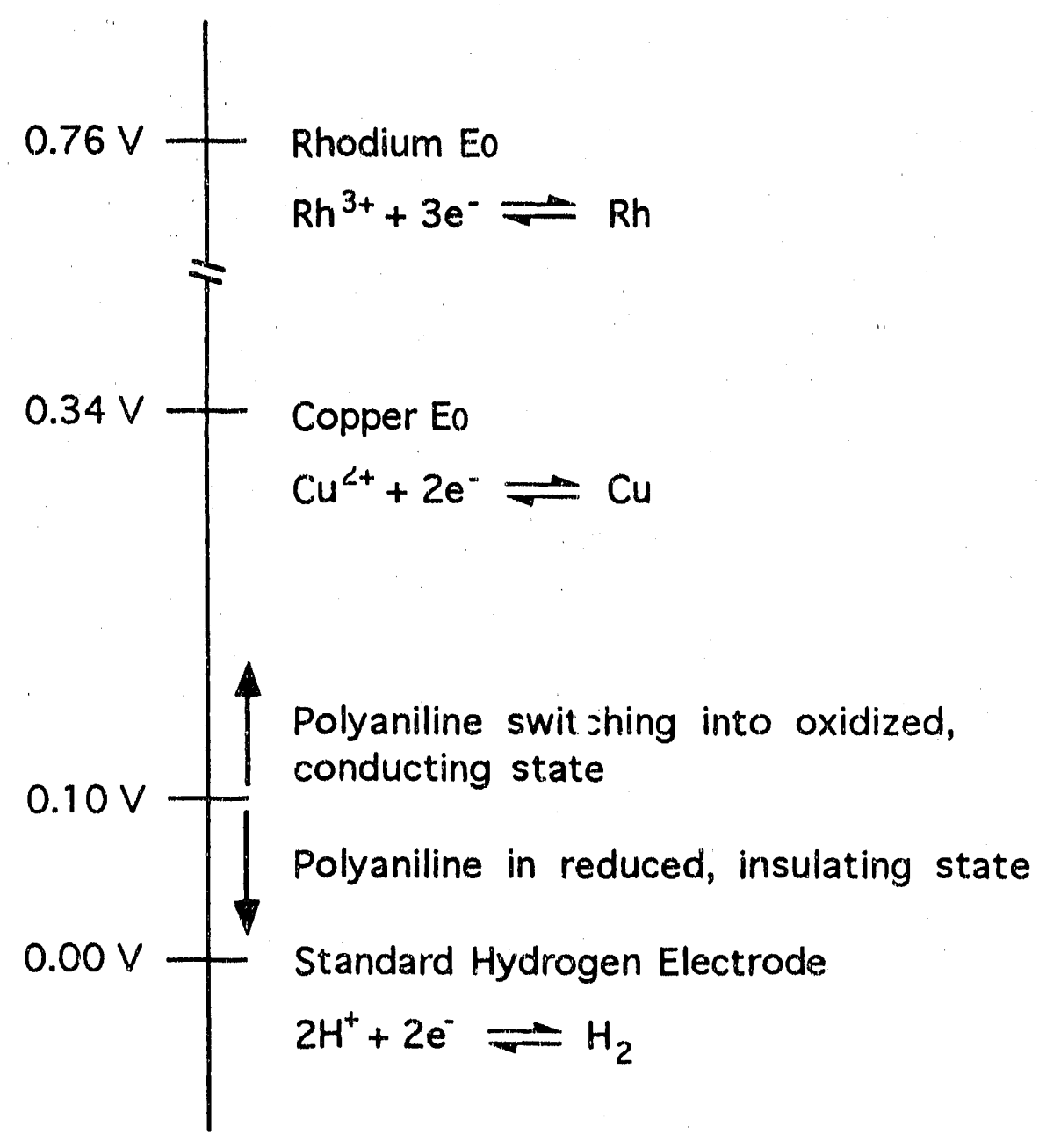

Figure 4-6: Potential scale indicating standard reduction potentials of copper and rhodium with respect to the standard hydrogen electrode and the polyaniline switching reaction. 'The PANI switching reaction occurs over a range of potentials between $+1 \mathrm{~V}$ and $+8 \mathrm{~V}$ versus hydrogen. PANI is found to be completely insulating below $+1 \mathrm{~V}$. 


\subsection{Conclusions:}

A few conclusions can be drawn from this preliminary study of copper deposition on polyaniline. Our initial investigation demonstrates the possibility of depositing copper on polyaniline. The nucleation density for the copper deposition must be increased before this system can be considered for thin multilayer applications. Many of the techniques proven to work on copper deposition on graphite should lead to similar improvements in nucleation densities of copper deposits on polyaniline, making the system a viable alternative to sputtered multilayers.

A different metal layer may also be helpful in producing better deposits. Rhodium can be deposited on the polymer in its conducting state with a larger overpotential than copper. Large overpotentials have been shown to lead to high nucleation densities on HOPG and the same is expected on the polymer.

Producing thin polymer layers with no pinholes has been found to be difficult. Thicker films have fewer pinholes; however, films of thicknesses on the order of only tens of nanometers are needed in multilayer structures for use in X-ray optics. 


\section{References:}

1 M.G. Kanatzidis, "Conductive Polymers", C\&EN 68:49, 36 (1990).

2 H. Shirakawa, E.J. Louis, A.G. MacDiarmid, C.K. Chiang, A.J. Heeger, "Synthesis of Electrically Conducting Organic Polymers: Halogen Derivatives of Polyacetylene", J. Chem. Soc., Chem. Commun., 16, 578 (1977).

3 A.F. Diaz, J.C. LaCroix, "Synthesis of Electroactive/Coaductive Polymer Films: Electrooxidation of Heteroaromatic Compounds", New Journal of Chernistry 12, 171 (1988).

4 A.F. Diaz, J.F. Rubinson, H.B. Mark, Jr., "Electrochemistry and Electrode Applications of Electroactive/Conductive Polynners", in Advances in Polymer Science 84, p. 113-139, edited by G. Flenrici-Olive and S. Olivê, Springer-Verlag, Berlin Heidelberg, 1988.

5 A.F. Diaz, J.A. Logan, "Electroactive Polyaniline Films" "J. Electroanal. Chem. 111, 111 (1980).

6 C.M. Carlin, L.J. Kepley, A.J. Bard, "Polymer Filmus on IElectrodes. XVI. In Situ Ellipsometric Measurements of Polybipyrazine, Polyaniline, and Polyvinylferrocene Films", J. Electrochem. Soc. 132, 353 (1985).

7 W.S. Huang, B.D. Humphrey, A.G. MacDiarmid ${ }^{\text {"Polyaniline, a Novel }}$ Conducting Polymer" , J. Chem. Soc., Faraday Trans. 182,2385 (1986).

8 E.M. Geniès, A. Boyle, M. Lapkowski, C. Tsintavis, "Polyaniline: A Historical Survey", Symthetic Metals 36, 139 (1990).

$9 \quad$ D.M. Mohilner, R.N. Adarns, W.J. Argersinger J Jr., "Investigation of the Kinetics and Mechanism of the Anodic Oxidation of Aniline in Aqueous Sulfuric Acid Solution at a Platinum Electrode", J. Am. Chem. Soc. 84, 3618 (1962). 
10 A. Kitani, J. Yano, K. Sasaki, "ECD Materials for the Three Primary Colurs Developed by Polyanilines", J. Electroanal. Chem. 209, 227 (1986).

11 T. Kobayashi, H. Yoneyama, H. Tamura, "Polyaniline Film-Coated Electrodes as Electrochromic Display Devices”, J. Electroanal. Chem. 161, 419 (1984).

12 A.G. MacDiarmid, J.C. Chiang, M. Halpern, W.S. Huang, S.L. Mu, M.L.D. Somasiri, W. Wu, S.I. Yaniger, "Polyaniline: Interconversion of Metallic and Insulating Forms", Mol. Cryst., Liq. Cryst. 121, 173 (1985).

13 E.M. Geniès, M. Lapkowski, "Electrochemical In-Situ EPR Evidence of Two Polaron-Bipolaron States in Polyaniline", J. Electroanal. Chem. 236, 199 (1987).

14 T. Kobayashi, H. Yoneyama, H. Tamura, "Electrochemical Reactions Concerned with Electrochromism of Polyaniline Film-Coated Electrodes”, J. Electroanal. Chem. 177, 281 (1984).

15 A. Kitani, J. Izumi, J. Yano, Y. Hiromoto, K. Sasaki, "Basic Behaviors and Properties of the Electrodeposited Polyaniline”, Bull. Chem. Soc. Japan 57, 2254 (1984).

16 T. Kobayashi, H. Yoneyama, H. Tamura, "Oxidative Degradation Pathway of Polyaniline Film Electrodes", J. Electroanal. Chem. 177, 293 (1984).

17 K. Okabayashi, F. Goto, K. Abe, T. Yoshida, "Electrochemical Studies of Polyaniline and its Application”, Synthetic Metals 18, 365 (1987).

18 A.F. Diaz, U. S. Patent No. 4,304,465, December 8,1981

19 A.F. Diaz, J.C. LaCroix, "Electroxidation of Aromatics to Polymer Films", Makromol. Chem., Macromol. Symp. 8, 17 (1987). 
20 E.M. Geniès, M. Lapkowski, J.F. Penneau, "Cyclic Voltammetry of Polyaniline: Interpretation of the Middle Peak", J. Electroanal. Chem. 249, 97 (1988).

21 M. Schirmeisen, F. Beck, "Electrocoating of Iron and other Metals with Polypyrrolen, J. Applied Electrochem. 19, 401 (1989).

22 S. Asavapiriyanont, G.K. Chandler, G.A. Gunawardena, D. Pletcher, "The Electrodeposition of Polypy. . ule Films from Aqueous Solutions”, J. Electroanal. Chem. 177, 229 (1984).

23 G.K. Chandler, D. Pletcher, "The Electrodeposition of Metals onto Polypyrrole Films from Aqueous Solution", J. Applied Electrochem. 16, 62 (1986).

24 J.Y. Lee, T.C. Tan, "Cyclic Voltammetry of Electrodeposition of Metal on Electrosynthesized Polypyrrole Film", J. Electrochem. Soc. 137, 1402 (1990).

25 V. Tsakova, A. Milchev, "Nucleation of Silver on a Polyaniline-coated Platinum Electrode", Electrochim. Acta 36, 1151 (1991).

26 Z.Q. Tian, Y.Z. Lian, J.Q. Wang, S.J. Wang, W.H. Li, " Electrochemical and XPS Studies on the Generation of Silver Clusters in Polyaniline Films", J. Electroanal. Chem. 308, 357 (1991).

27 S. Mazur, S. Reich, "Electrochemical Growth of Metal Interlayers in Polyimide Film", J. Phys. Chem. 90, 1365 (1986).

28 G.T. Dee, L.E. Manring, S. Mazur, "Kinetics of Metal Interlayer Growth in Polyimide Films: Metal Distributions in the Non-steady State Regime and with Constraints of Patterned Boundaries", J. Phys. Chem. 91, 6699 (1987).

29 M. Levy, L.E. Manring, S. Mazur, "Electron Carrier Mediated Deposition of Metal Interlayers in Polymer Films", J. Electrochem. Soc. 135, 2479 (1988) 


\section{Chapter 5:}

\section{SUMMARY AND CONCLUSIONS}

The electrodeposition of copper on graphite electrodes was studied, with special attention given to the nucleation phenomenon. The nucleation density of copper on graphite was found to be four orders of magnitude lower than that on platinum under similar deposition conditions. ${ }^{1}$ To achieve the high nucleation densities required for producing thin, continuous films, we must first understand how various deposition conditions affect the nucleation phenomenon. The results from this investigation can serve as a guide for metal deposition onto other low electron density substrates, such as conducting polymers and semiconductors.

\subsection{Determination of Nucleation Density:}

For the study of the effect various deposition conditions have on nucleation density, a reliable method for determining the number density of nuclei is needed. Various ex-situ and in-situ techniques were investigated. The two direct methods employed, which involve counting the number of nuclei imaged, were: scanning electron microscopy and the scanning tunneling microscopy. The indirect determinations included Raman spectroscopy and the analysis of potentiostatic current transients.

Even though the bare graphite surfaces could be imaged over a wide range of magnifications with the STM, the copper nuclei deposited onto graphite could not be imaged. A possible explanation is that the nodules are not firmly attached to the surface and interaction with the probe tip causes them to move. When the nodules were densely packed, however, the STM was able to image them. The resiriction to 
high copper coverages limits the value of the STM as a tool for determining nucleation densities in the copper-graphite system.

A clear decrease in the intensity of the Raman signal from graphite was observed with increased copper coverage. Optical models were developed to determine the copper nucleation density from the attenuation of the graphite Raman intensity. The models rely on an empirical relationship to match the SEM measured number densities. Though the models were able to successfully predict the nucleation density, problems associated with long data collection times and laser enhanced deposition limited the use of Raman as an in-situ tool.

To determine the nucleation density from the potentiostatic current transient, the Scharifker and Mostany ${ }^{2}$ method was employed. This approach uses the Fleischmann and Thirsk ${ }^{3}$ nucleation model coupled with mass transport limited growth of the nuclei and statistically determined overlap of the concentration depletion zones. The model fits the experimentally measured current response to a potential step; however, the predicted nucleation densities are much lower than those measured with the SEM.

Though some of the above techniques were shown to correctly predict the nucleation densities under special conditions, SEM was found to be the most reliable tool. Some problems with experimental scatter and reproducibility necessitated averaging the nucleation densities counted on a minimum of fifteen locations on every sample. The large scatter has been attributed to surface variations and possible material redistribution from small to large nodules. Steps to minimize the effect of these problems, such as careful sample preparation and quick removal of the graphite surface from electrolyte after the completion of copper deposition, were implemented. 


\subsection{Experimental Results:}

To study how strongly nucleation is governed by the properties of the substrate, a quantitative determination of the differences between deposition on graphite and on a metal electrode, such as platinum, was required. For copper electrodeposition, the existence of a 250 millivolts nucleation overpotential on graphite was measured with cyclic voltammetry. Such a large overpotential does not occur on a platinum or on a copper covered graphite electrode. The nucleation overpotential is indicative of an activation barrier to copper nucleation on HOPG.

Of all of the variables examined, the deposition potential is found to be the dominant parameter governing nucleation density. There is a sharp increase in the nucleation density with applied potential. Copper can be deposited on HOPG only over a limited range of potentials, above the nucleation overpotential and below the potential where hydrogen evolution results in surface disordering. Other researchers have determined that the electrochemical activity of graphite electrodes can be increased by increasing the number of surface defect sites. In this research, we have concentrated on determining the variables that increase the nucleation density, which can be extended to other systems; therefore, we have avoided conditions that may activate the graphite surface but are not applicable to other systems.

To increase the copper nucleation density, while avoiding excessive hydrogen evolution, a double pulse potential technique was applied. The surface was nucleated with a short, high potential pulse, and the nuclei grown at a lower potential, where hydrogen evolution does not occur. The growth period is followed by a period where no copper is deposited to allow the concentration field to relax. The procedure is then repeated with an increase in the nucleation density with each 
consecutive pulse. Nucleation densities on the order of $10^{10}$ nuclei $/ \mathrm{cm}^{2}$ were obtained using this technique.

Another possible way to control electrodeposition is by using chemical additives, such as inhibitors, in the electrolyte. Polyvinyl alcohol and benzotriazole were examined to determine the effect they have on the nucleation density. Polyvinyl alcohol inhibited both the nucleation and the growth reactions of copper on HOPG. Benzotriazole was found to more selectively inhibit the growth of the nodules.

\subsection{Possible Extensions of Present Study:}

A natural extension of the nucleation study presented in this thesis would be to study metal deposition on other low electron density surfaces such as conducting polymers. The initial investigation of depositing copper on polyaniline, shows that the system is a viable one. More research is needed to produce thin polymer films which have fewer pinholes and to increase the nucleation density of copper on the polymer layer. Many of the techniques proven to work on increasing the nucleation density of copper on graphite should lead to similar improvements in nucleation densities on polyaniline, making the electrochemical production of thin multilayer structures a possibility.

A metal other than copper may be needed for producing metal layers with high nucleation densities. The restriction on the choice of metal comes from the switching potential of the polymer. Polyaniline is in its non-conducting form at potentials were many metals reduce, making it impossible to deposit some metals on it. Metals more noble than copper, such as rhodium can be deposited with a larger overpotential, while still keeping the polymer in its conducting state. Large 
overpotentials have been shown to lead to high nucleation densities on HOPG; the same is expected on the polymer.

\section{References:}

1 M. J. Armstrong, "The Role of Inhibitors During Electrodeposition of Thin Metallic Films", Ph.D. Dissertation, University of California, Lawrence Berkeley Laboratory, LBL-28972 (1990).

2 B.R. Scharifker, J. Mostany, "Three-Dimensional Nucleation with Diffusion Controlled Growth, Part I. IJumber Density of Active Sites and Nucleation Rates per Site”, J. Electroanal. Chem. 177, 13 (1984).

3 M. Fleischmann, H.R. Thirsk, "Anodic Electrocrystallization", Electrochim. Acta 2, 22 (1960). 


\section{Appendix A:}

Computer code in $\mathrm{C}$ for solving the set of two equations from the

Scharifker Mostany nucleation and growth model using the Newton-Raphson technique. (Details in section 2.3)

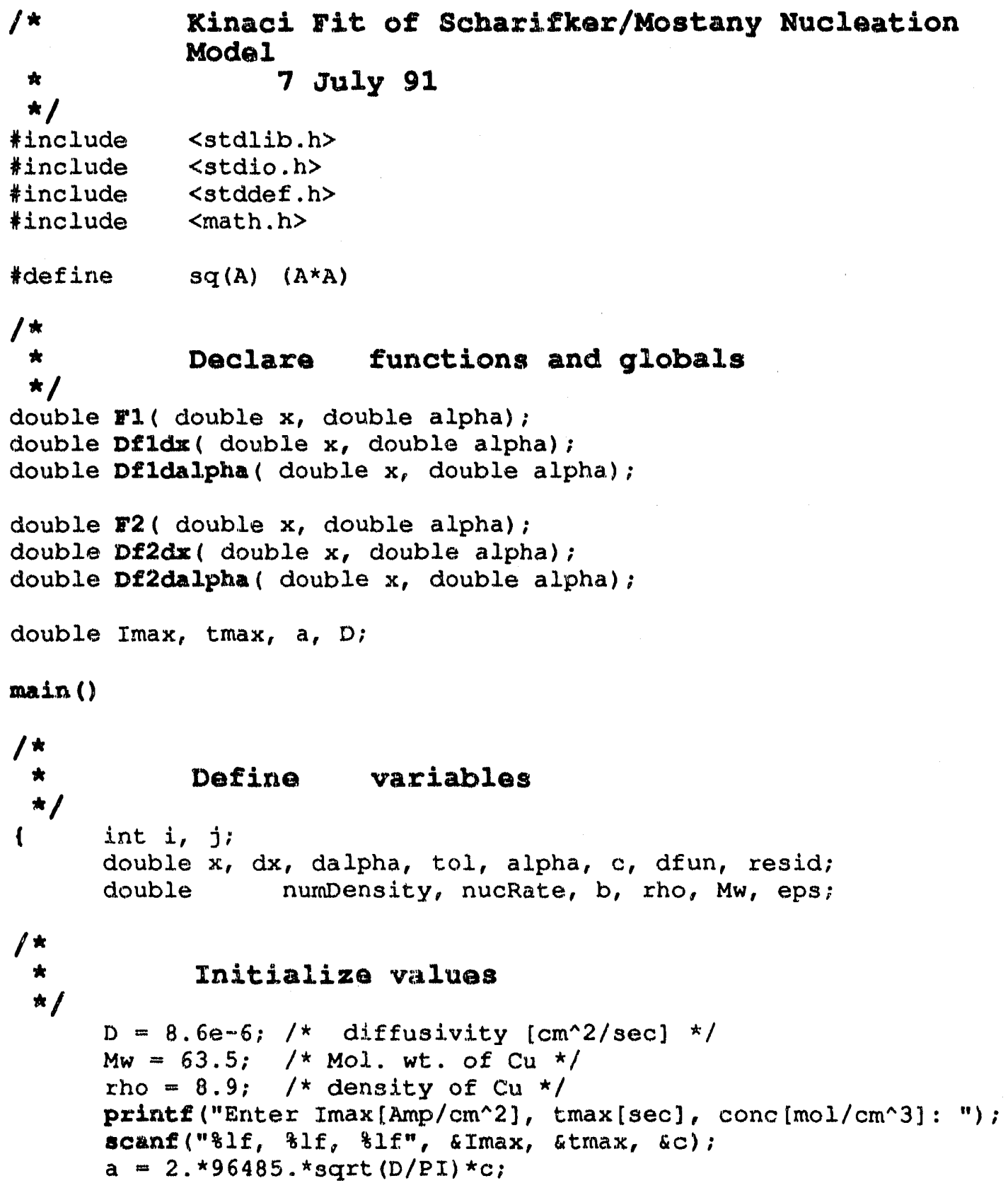




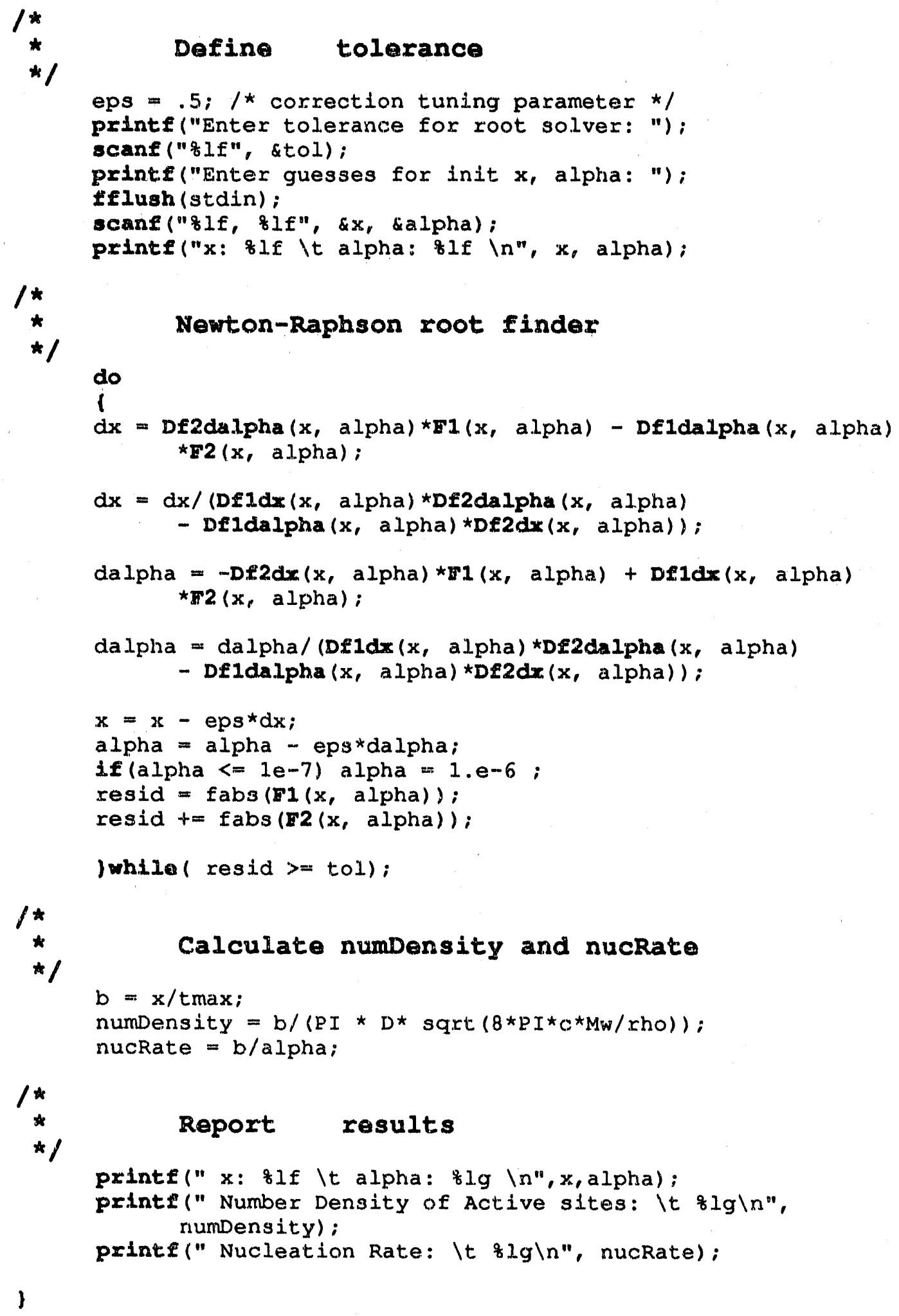




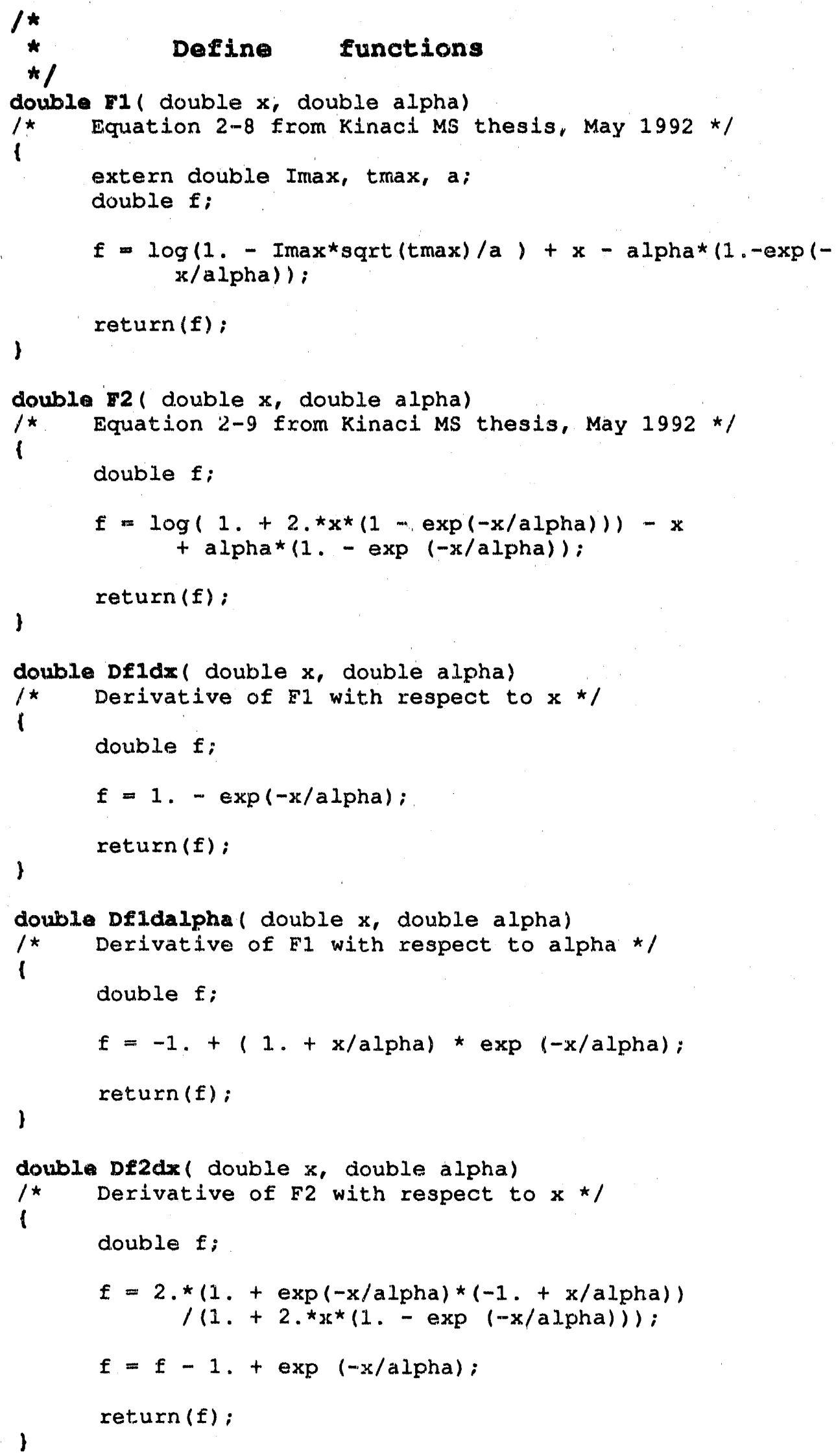


double Df2dalpha ( double $*$, double alpha)

/* Derivative of $F 2$ with respect to alpha */

1

double $\mathrm{f}$;

$f=-2 \cdot{ }^{*} \mathrm{sq}(\mathrm{x} / \mathrm{alpha}) * \exp (-\mathrm{x} / \mathrm{alpha})$

$/\left(1 .+2 . * x^{*}(1 .-\exp (-x /\right.$ alpha $\left.))\right) ;$

$f=f+1 .-\exp (-x / a l p h a) *(1 .+x / a l p h a) ;$

3

return (f); 


\section{Appendix B:}

Computer code in FORTRAN for solving the relative Raman intensity of a graphite surface coated with a nodular copper layer (islands) versus the bare graphite surface. (Details in Section 2.5)

BLOCK DATA

$\begin{array}{lll}\text { C } & \text { DATA } & \text { FOR INCIDENT AND SCATTERED BgAMS } \\ \text { C } & \text { PHI } & \text { Angle of Incidence in rad. } \\ \text { C } & \text { WL } & \text { Wavelength in CM. } \\ \text { C } & \text { TNA } & \text { Ambient Refractive Index, } 1.34 \text { for Water } \\ \text { C } & \text { TNCU } & \text { Complex RI for Copper }(k<0) \\ \text { C } & \text { TNGR } & \text { Complex RI for Graphite } \\ \text { C } & & \end{array}$

COMMON /INC/ RHII, WLI, TNA, TNCUI, TNGRI

COMMON /SCAT/ PHIS, WLS, TNCUS, TNGRS

REAL $\star 8$ PHII, PHIS, TNA, WLI, WLS

COMPLEX 16 TNCUI, TNGRI, TNCUS, TNGRS

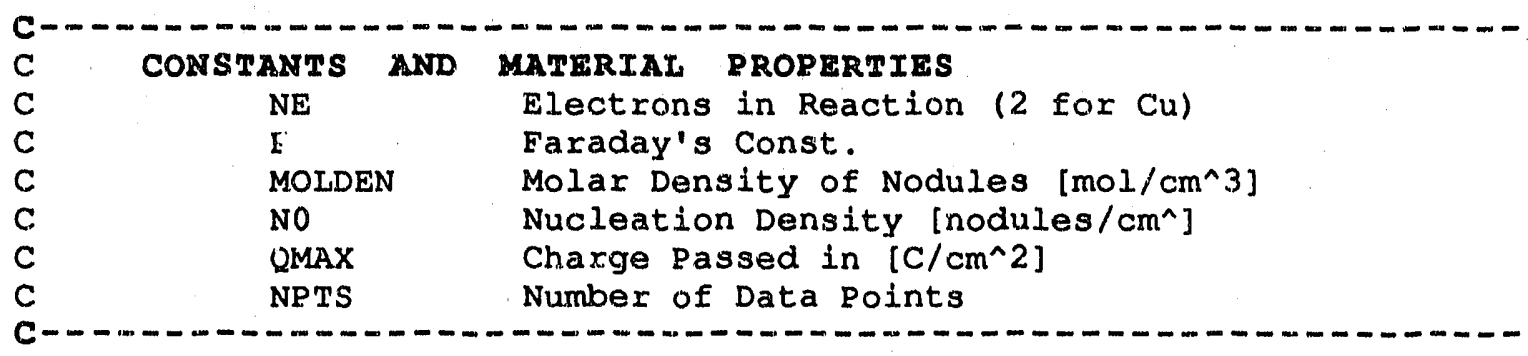

COMMON /CONST/ NE, F, MOLDEN, NO, PI, QMAX, NPIS

REAL*8 F, MOLDEN, NO, PI, QMAX

INTEGER NE, NPTS

DATA PHII, PHIS $/ 0 ., .7853982 /$

DATA WLI, WLS, TNA/ 488.E-7, 529.E-7,1.34/

DATA TNCUI, TNCUS / $(1.14,-2.53),(1.08,-2.59) /$

DATA TNGRI, TNGRS/ $(2.55,-1.37),(2.58,-1.43) /$

DATA NE, F,MOLDEN, N0 /2, 96487., .1411, 1.E8/

DATA PI, QMAX, NPTS / 3.141596, 0.01, 10/

END

PROGRAM RAMCS3

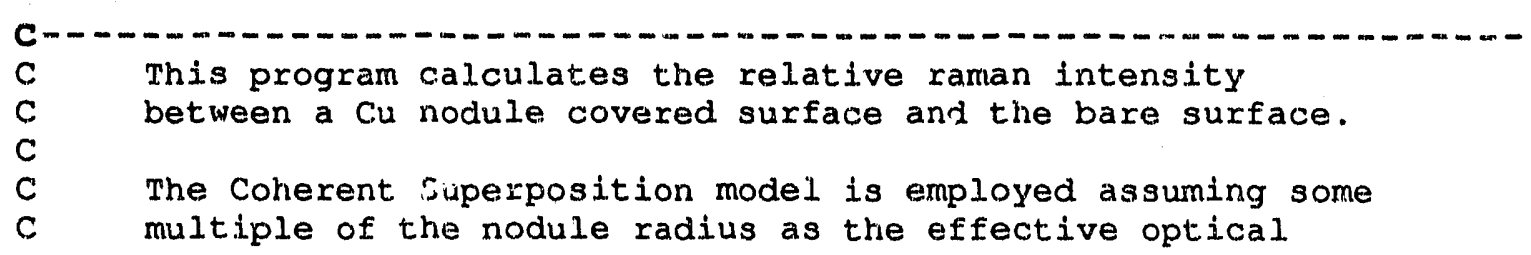




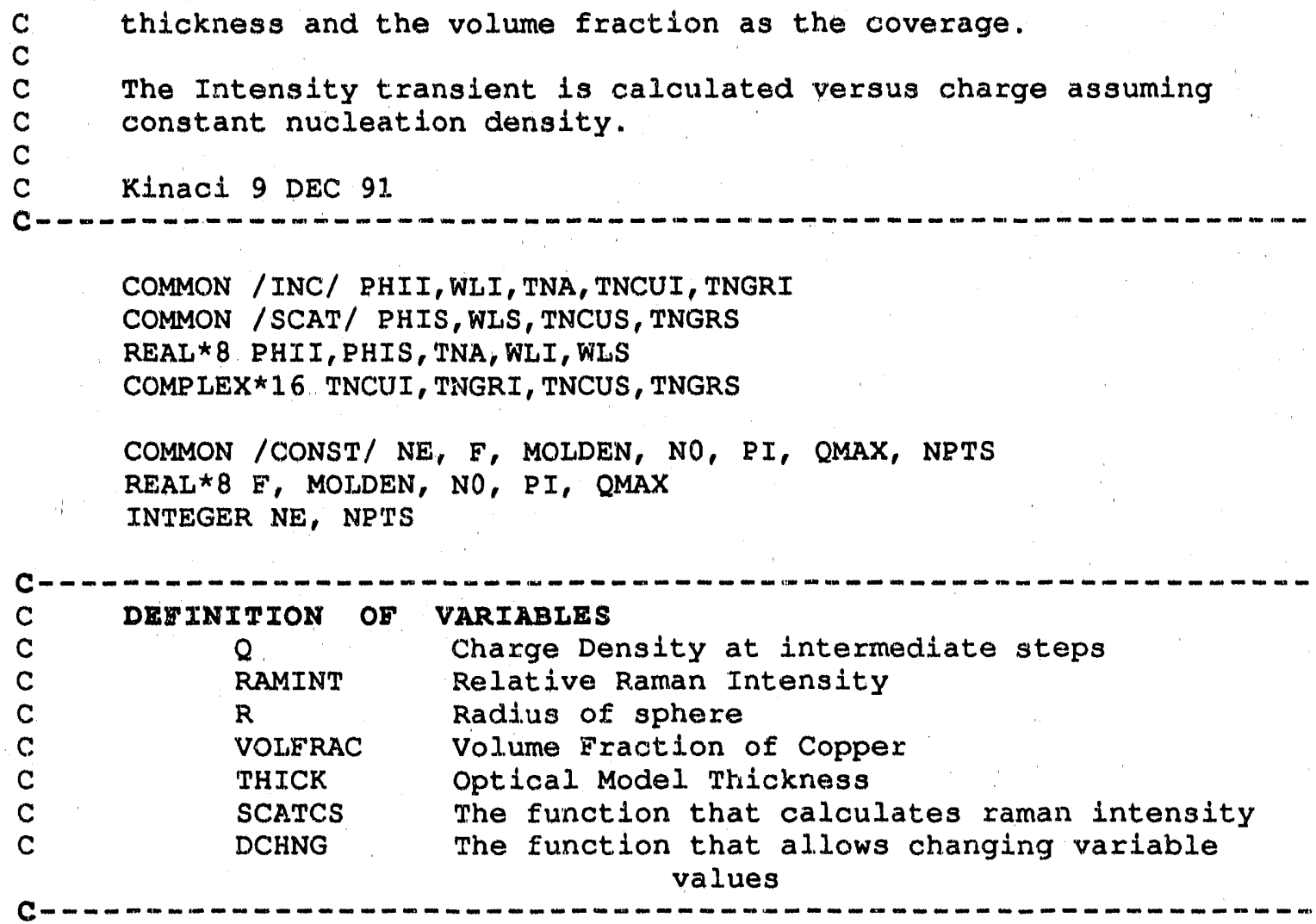

REAL*8 Q, RAMIN!, R, VOLERAC, THICK

REAL * 8 SCATCS, DCHNG

INTEGER I

CHARACTER *64 FILENAME

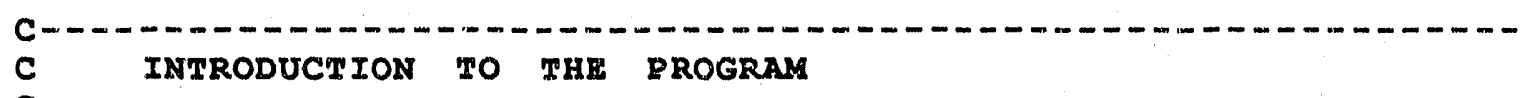

WRITE (*,*) 'This progran calculates the relative Raman' WRITE $(*, *)$ 'intensity vs. charge for const. No using'

WRITE $(*, *)$ 'the coherent superposition model.'

MRIXR $(*, *)$ 'The thickness = Empirical function of the'

HRITE $(*, *)$ ' Radius of Hemisphere'

WRITE (*,*) 'The coverage = volume fraction $\mathrm{Cu}$ in layer'

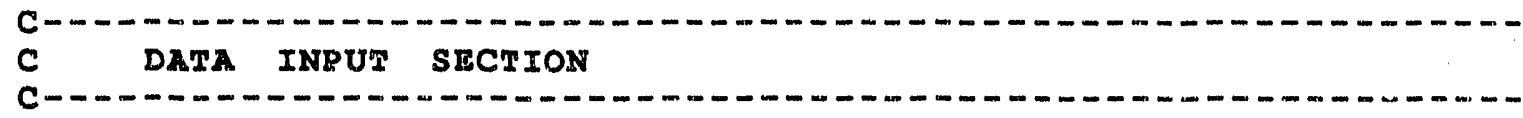

MRITR $(*, *)$ 'Enter the Nucleation Density (per sq. cm)' NO $=$ DCHNG (NO)

WRITE $(*, *)$ 'Enter the amount of charge passed(C/sq.cm):' QMAX $=$ DCXNG (QMAX)

WRITd (*,*) 'Enter the number of data points:' 
NPTS $=$ ICHNG (NPTS)

MrIrm (*, 'Enter the file nams for the data:'

10

READ $(*, 10)$ EILENAME

10 FORMAI (A64)

ORIN (UNIT=1, EIIE $=$ F ILENAME)

\section{CaLCOLATION Or Transiants}

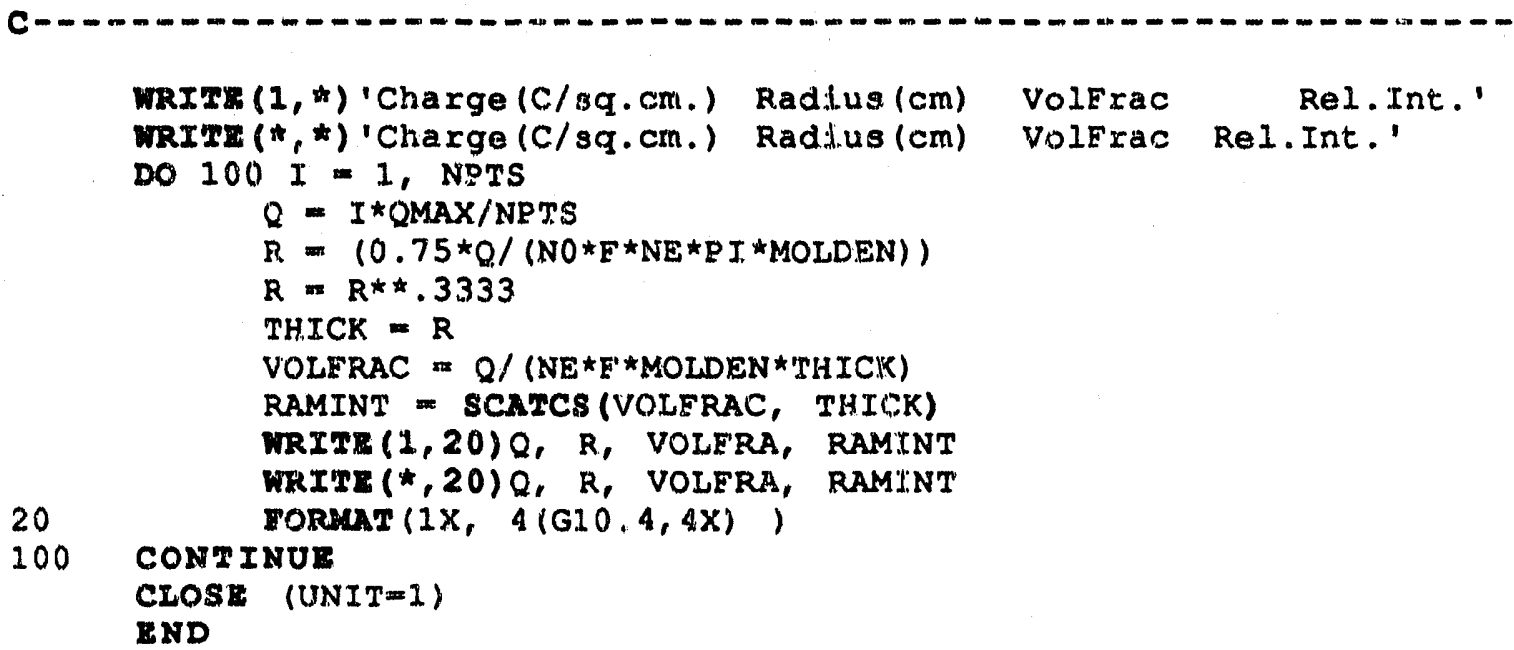


DOUBLE PRECISION FUNCTION SCAYCS (VOLERAC, THICK)

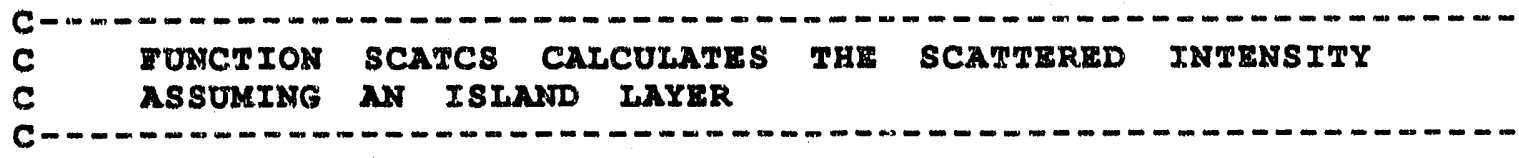

REAL * 8 VOLFRAC, THICK

COMMON /INC/ PHII, WII, TNA, TNCUI, TNGRI

COMMON /SCAT/ PHIS, WLS, TNCUS, TNGRS

REAL* 8 PHII, PHIS, TNA, WLI, WLS

COMP LEX 16 INCUI, TNGRI, TNCUS, TNGRS

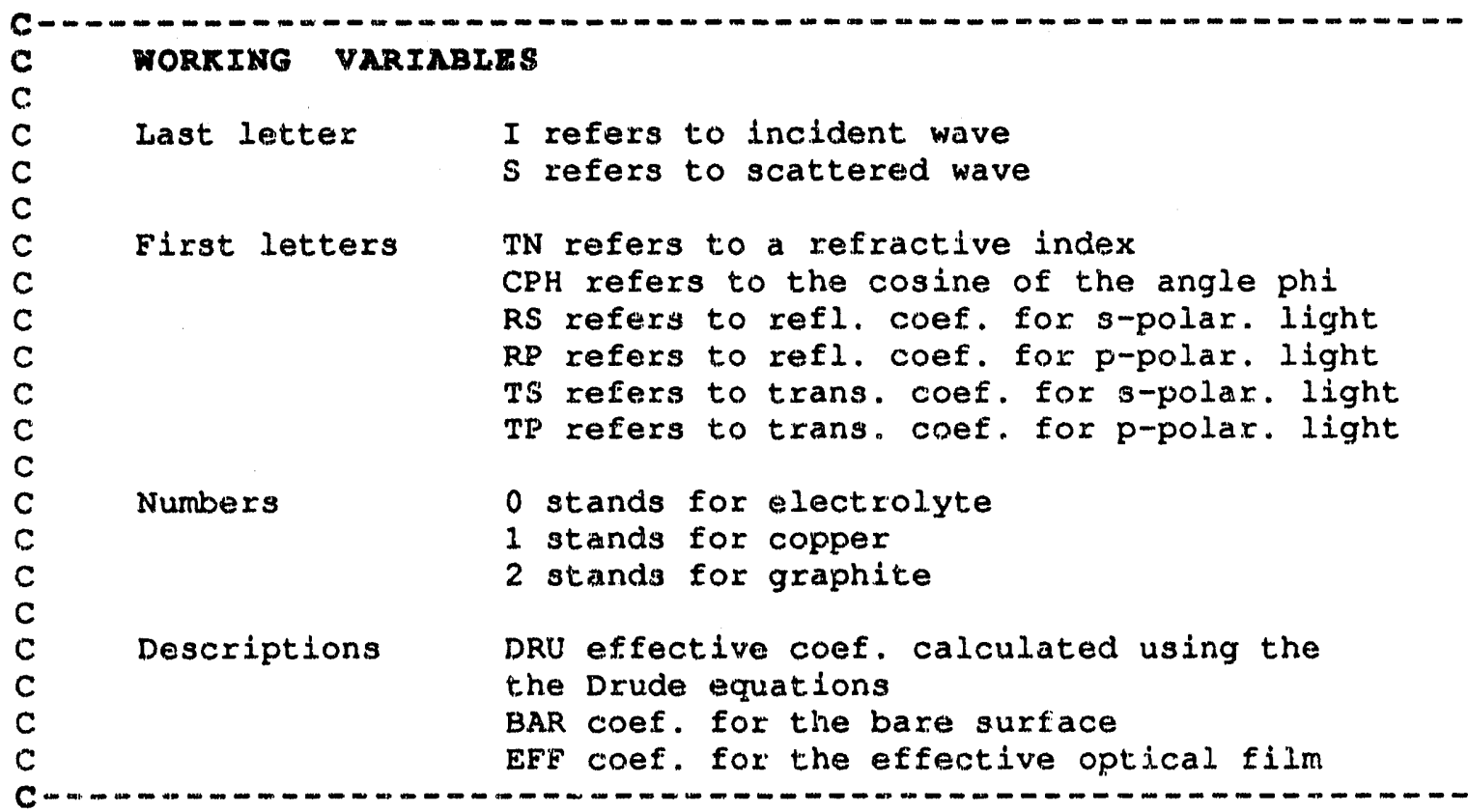

COMPLEX*16 TNO

COMPLEX*16 RS01, RP01, TS01, TP01

COMPIUE* 16 RS02, RP02, TS02, TPO2

COMPIEX*16 RS12,RP12,TS12,TP12

COMP LEX 1.6 RS10, RP10, TS10,TP10

COMPLEX $* 16$ RS20, RP20, TS20,TP20

COMPLEX 16 RS21, RP21, TS21, TP21

COMPLEX 16 RSDRUI, TSDRUI, RSDRUS, TSDRUS

COMPLEX ${ }^{*} 16$ TSBARI, TSBARS

COMPIJEX*16 TSEFFI, TSEFES

COMPLEX*16 TSCAT

COMPIEX*I 6 CPHII, CPHCUI, CPHGRI, CPHIS, CPHCUS, CPHGRS

$T N O=D C M P I X X(T N A, 0$.

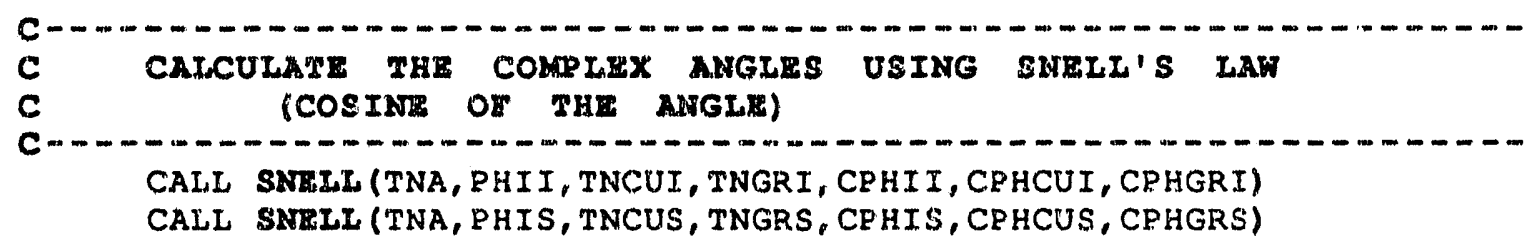




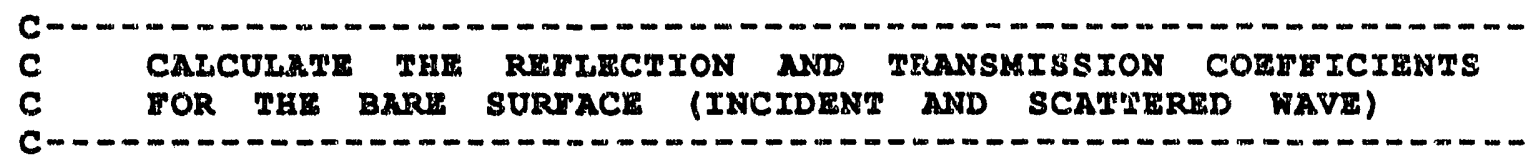

CALI WRESHEJ (CPHII, CPHGRI, TNO, TNGRI, RS02, RP02, TS02, TP02) TSBARI $=$ TSO2

CALI YRESWEL (CPHGRS, CPHIS, TNGRS, TNO, RS20, RP 20, TS20, TP20)

TSBARS $=$ TS20

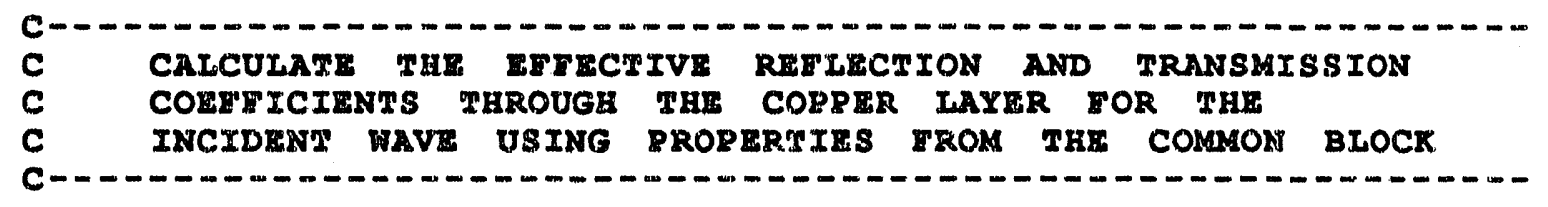

CALL PRESAEL (CPHII, CPHCUI, TNO, TNCUI, RSO1, RP01, TSO1, TP01)

CAII WESART (CPHCUI, CPHGRI, TNCUI, TNGRI, RS12, RP12, TS12, TP12)

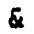

CALL DRUDEP (WLT, TNCUI, CEHCUI, THICR, RSO1, RS12, TSO1, TS12,

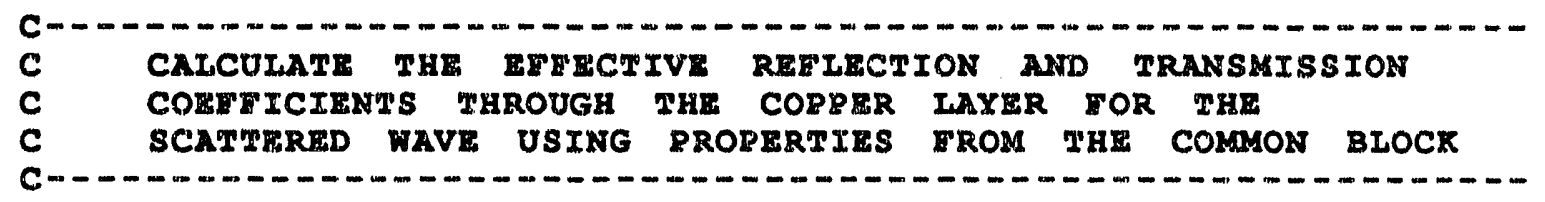

CALI TRESNZI (CRHGRS, CPHCUS, TNGRS, TNCUS, RS21, RP 21, TS21, TP21)

CALI TRBSNEI (CPHCUS, CPHIS, TNCUS, TNO, RS10, RP10, TS10, TP10)

CALI DRUDEN (WLS, TNCUS, CPHCUS, THICK, RS21, RE10, TS21, TS10,

\& RRDRUS, TSDRUS)

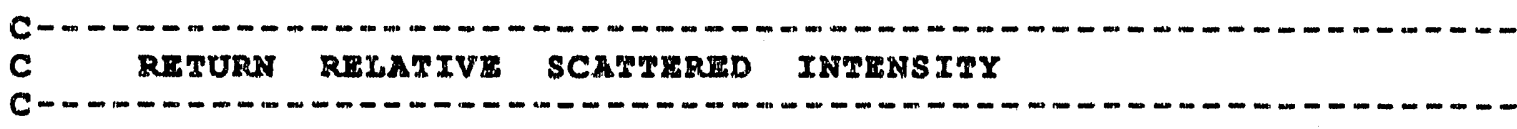

TSEFET $=$ CSQRT (VOLFRAC *TSDRUI**2+(1-VOLPRAC) *TSBARI**2)

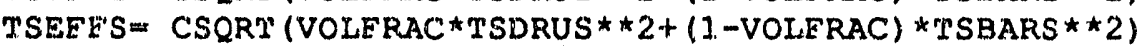

TSCAT $=$ TSEFE I*TSEFES / (TSBARI*TSBARS)

SCATCS $=$ 'SSCAT * CONJG (TSCAT)

RETURN

END 
SUBROUTINR SNRLI (TNO, RHIO, TN1, TN2, CPHIO, CPHI1, CPHI2)

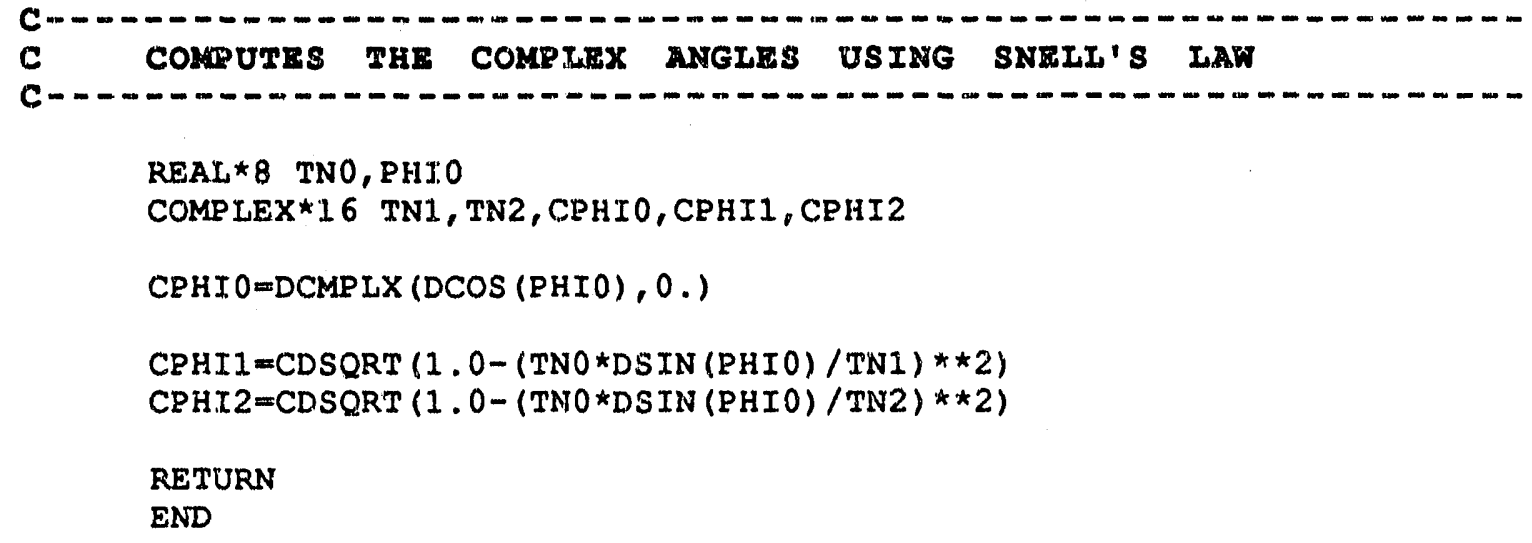



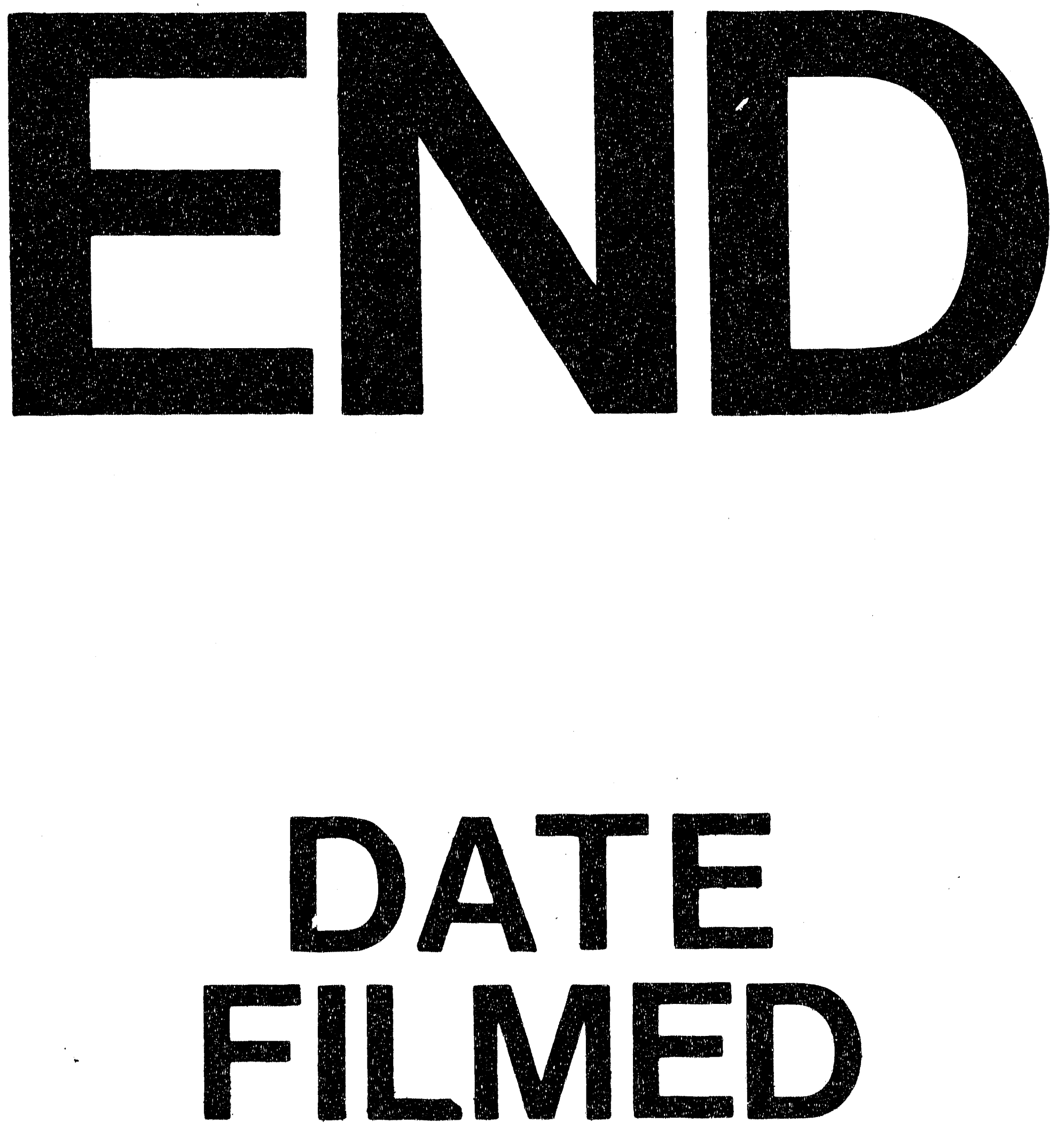

䇐

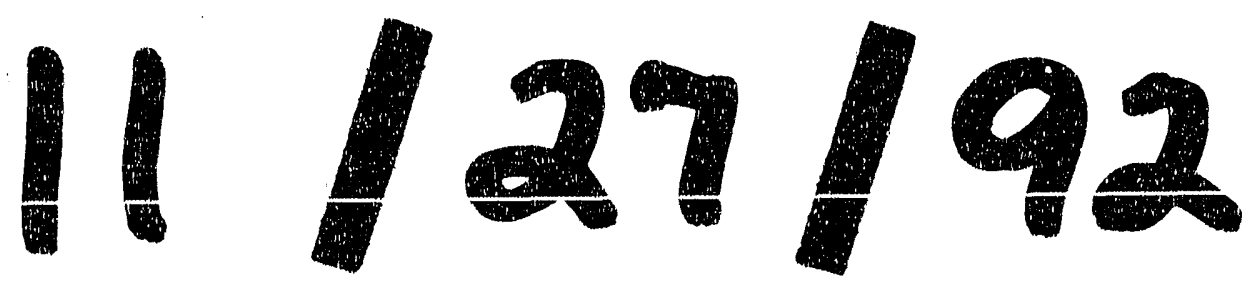


